\title{
ON THE SPECTRAL DECOMPOSITION OF AFFINE HECKE ALGEBRAS
}

\author{
ERIC M. OPDAM
}

\begin{abstract}
An affine Hecke algebra $\mathcal{H}$ contains a large abelian subalgebra $\mathcal{A}$ spanned by the Bernstein-Zelevinski-Lusztig basis elements $\theta_{x}$, where $x$ runs over (an extension of) the root lattice. The center $\mathcal{Z}$ of $\mathcal{H}$ is the subalgebra of Weyl group invariant elements in $\mathcal{A}$. The natural trace ("evaluation at the identity") of the affine Hecke algebra can be written as integral of a certain rational $n$-form (with values in the linear dual of $\mathcal{H}$ ) over a cycle in the algebraic torus $T=\operatorname{spec}(\mathcal{A})$. This cycle is homologous to a union of "local cycles". We show that this gives rise to a decomposition of the trace as an integral of positive local traces against an explicit probability measure on the spectrum $W_{0} \backslash T$ of $\mathcal{Z}$. From this result we derive the Plancherel formula of the affine Hecke algebra.
\end{abstract}

Date: November 14, 2018.

1991 Mathematics Subject Classification. 20C08, 22D25, 22E35, 43A32.

The author would like to thank Erik van den Ban, Patrick Delorme, Gerrit Heckman, Mark Reeder, Klaas Slooten and Atsuko Yamamoto for their helpful comments and remarks. 


\section{Contents}

1. Introduction 3

2. Preliminaries and description of results 8

2.1. The affine Weyl group and its root datum 9

2.2. Parabolic subsystems 11

2.3. Root labels 11

2.4. The Iwahori-Hecke algebra as a Hilbert algebra 12

2.5. Bernstein's description of the center $\mathcal{Z} \quad 15$

2.6. Positive elements and positive functionals 18

$\begin{array}{ll}\text { 2.7. Casselman's criteria } & 19\end{array}$

2.8. The Plancherel measure 22

2.9. Outline of the main results 23

3. Localization of $\tau$ on $\operatorname{Spec}(\mathcal{Z}) \quad 28$

3.1. $\omega$-residual cosets 30

3.2. The contour shift and the local contributions 32

3.3. Application to the trace functional 42

3.4. The Plancherel measure $\nu$ of $\mathcal{Z}$, and the $\mathcal{A}$-weights of $\chi_{t} \quad 48$

3.5. Discrete series $\quad 55$

3.6. Temperedness of the traces $\chi_{t} \quad 57$

4. Localization of the Hecke algebra 58

4.1. Lusztig's structure theorem and parabolic induction 61

4.2. The tracial states $\chi_{t}$ and parabolic induction 69

4.3. The generic residue of the Hecke algebra 74

4.4. Unitarity and regularity of intertwining operators 80

4.5. The Plancherel decomposition of the trace $\tau \quad 82$

5. Base change invariance of the residual algebra 93

5.1. Scaling of the root labels 93

5.2. Application to the residual algebras 96

6. Applications and closing remarks 98

6.1. Formation of L-packets of unipotent representations 98

6.2. Operator norm estimate and the Schwartz completion 100

6.3. A Hilbert algebra isomorphism; abelian subalgebras 104

6.4. Central idempotents of $\mathfrak{C}$ and $\mathfrak{S} \quad 105$

6.5. Some examples 107

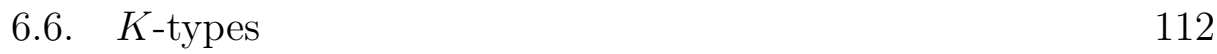

6.7. A remark on the residual degrees $d_{\pi} \quad 113$

7. Appendix: Residual Cosets 114

7.1. Introduction and quick guide 114

7.2. Definition and Classification of Residual Cosets 116

7.3. Properties of residual and tempered cosets 120

8. Appendix: Kazhdan-Lusztig parameters 124 


\section{Introduction}

In this paper we will discuss the spectral decomposition of an affine Hecke algebra $\mathcal{H}$ defined over $\mathbb{C}$ or, more precisely, of a natural positive trace $\tau$ defined on $\mathcal{H}$. In the standard basis of $\mathcal{H}, \tau$ is simply defined by $\tau\left(T_{e}\right)=1$ and $\tau\left(T_{w}\right)=0$ if $w \neq e$. In addition, $\mathcal{H}$ comes equipped with the natural $*$-operator $T_{w}^{*}=T_{w^{-1}}$. This defines a preHilbert structure on $\mathcal{H}$ by $(x, y):=\tau\left(x^{*} y\right)$. The regular representation $\lambda \times \rho$ extends to the Hilbert completion $\mathfrak{H}$ of $\mathcal{H}$, and by the spectral decomposition of $\tau$ we mean the decomposition of $\mathfrak{H}$ in irreducible $*$ representations of $\mathcal{H} \times \mathcal{H}$. By classical results on the decomposition of traces on $C^{*}$-algebras of type I (see for example [15]), this is equivalent to the problem of decomposing the trace $\tau$ as a superposition of irreducible characters of $*$-representations of $\mathcal{H}$. We will call this decomposition the Plancherel decomposition of $\mathcal{H}$, and the associated positive measure on the spectrum $\hat{\mathcal{H}}$ will be called the Plancherel measure.

In the case of a Hecke algebra of finite type we have the well known decomposition formula

$$
\tau=\frac{1}{P} \sum \chi_{\pi} d_{\pi}
$$

where $P$ denotes the Poincaré polynomial of $\mathcal{H}$ (we assume that $P \neq 0$ ), $\pi$ runs over the finite set of irreducible representations of $\mathcal{H}, \chi_{\pi}$ denotes the corresponding character of $\pi$, and $d_{\pi}$ is the generic degree of $\pi$. The formula we are going to discuss in the present paper is the affine analog of equation (1.1).

This paper is the sequel to [37], where we made a basic study of the Eisenstein functionals of an affine Hecke algebra $\mathcal{H}$. These Eisenstein functionals are holomorphic functions of a spectral parameter $t \in T$, where $T$ is a complex $n$-dimensional algebraic torus naturally associated to $\mathcal{H}$. In [37], we derived a representation of $\tau$ as the integral of the normalized Eisenstein functional times the holomorphic extension of the Haar measure of the compact form of $T$, against a certain "global $n$-cycle" (a coset of the compact form of $T$ ) in $T$. The kernel of this integral is a meromorphic $(n, 0)$-form on $T$.

The present paper takes off from that starting point, and refines step-by-step the above basic complex function theoretic representation 
formula for $\tau$ until we reach the level of the spectral decomposition of $\tau$, extended to a tracial state on the $C^{*}$-algebra hull $\mathfrak{C}$ of the regular representation of $\mathcal{H}$ (Main Theorem 4.43). On the simpler level of the spherical or the anti-spherical subalgebra, a similar approach can be found in [34] and [19]. In the case of the spherical algebra one should of course also mention the classical work [31], although the point of view is different there, and based on analysis on a reductive $p$-adic group.

1.0.1. Motivation. There are various motivations for the study of the spectral resolution of $\tau$. A natural application of such a decomposition is the p-adic analog of the Howlett-Lehrer theory for finite reductive groups, see for instance [36], [28], [19], [39] and [40]. Here one considers an affine Hecke algebra which arises as the centralizer algebra of a certain induced representation of a p-adic reductive group $G$. The Plancherel measure of $\mathcal{H}$ can be interpreted as the Plancherel measure of $G$ on a part of $\hat{G}$ in this situation. In view of this application it is important that we obtain an (almost) explicit product formula for the Plancherel measure (see Main Theorem 4.43). In addition we characterize exactly which characters $W_{0} r \in W_{0} \backslash T$ of the spectrum of the center $\mathcal{Z}$ of $\mathcal{H}$ support a discrete series representation of $\mathcal{H}$ (see Theorem 3.29). These are the so-called "residual points" (see Appendix 7 ). This result was recently applied to the representation theory of reductive p-adic groups, see [20].

Another motivation for this approach is that it sets the stage for the definition of a Schwartz-completion $\mathfrak{S}$ of $\mathcal{H}$ (see Subsection 6.2), and for the subsequent study of the Fourier transform and its inversion on the level of this Fréchet algebra (joint work with Patrick Delorme, to appear). This is related to the study of the K-theory and the cyclic homology of $\mathcal{H}$ and its reduced $C^{*}$-algebra $\mathfrak{C}$, in the spirit of [50], [51]. This point of view is particularly interesting for non-simply laced cases, since it is natural to expect that the K-theory does not depend on the parameters $q(s)$ of the Hecke algebra. On the other hand, in the "generic case" these matters seem to be considerably easier to understand than in the "natural cases", where the logarithms of the parameters have rational relations. In view of this, it is important that we allow the parameters $q(s)$ of the affine Hecke algebra to assume any real value $>0$.

1.0.2. Outline. It may be helpful to give the reader a rough outline of this paper, and an indication of the guiding principles in the various stages. We also refer the reader to Subsection 2.9 for a more detailed 
outline and formulation of the main results (see in particular 2.9.4, 2.9.5 for the results on the Plancherel measure).

(0). The starting point of the present paper is the definition of the Eisenstein functional of the affine Hecke algebra, in [37]. These functionals are matrix coefficients of minimal principal series modules. The study of their intertwining operators led to a representation of the trace $\tau$ of $\mathcal{H}$, as an integral of a certain rational kernel over a "global" cycle (see formula (3.1)).

(1). In section 2 we recall the definition and first properties of (extended) affine Hecke algebras, we collect some basic facts from the theory of $C^{*}$-algebras, and we adapt certain classical results from the representation theory of reductive groups to our context. We conclude this section with a discussion of the properties of the natural map $p_{z}: \hat{\mathfrak{C}} \rightarrow \operatorname{Spec}(\mathcal{Z})$, the spectrum of the center $\mathcal{Z}$ of $\mathcal{H}$, in view of the main results of this paper.

(2). The study of the residues of the rational kernel for $\tau$ as in formula (3.1), in Section 3. This involves a general (but basic) scheme for the calculation of multivariable residues. After symmetrization over the Weyl group, the result is a decomposition of $\tau$ as an integral of local tracial states against an explicit probability measure on the spectrum $\operatorname{Spec}(Z)=W_{0} \backslash T$. The main tools in this process are the positivity of $\tau$, and the geometric properties of the collection of residual cosets (Appendix 7). This step is called the "localization of the trace $\tau$ ".

(3). The local trace (as was mentioned in (2)) defined at an orbit $W_{0} t \subset T$, arises as an integral of the Eisenstein kernel over a "local cycle" which is defined in an arbitrarily small neighborhood of the orbit $W_{0} t$. This gives a natural extension of the local trace to localizations of the Hecke algebra itself (localization as a module over the sheaf of analytic functions on $\left.W_{0} \backslash T\right)$.

The analytic localization of the Hecke algebra has a remarkable structure discovered by Lusztig in [26]. This part of the paper is not selfcontained, but draws heavily on the paper [26]. By Lusztig's wonderful structure theorem we can now investigate the local traces. We find in this way that everything is organized in accordance with HarishChandra parabolic induction (the philosophy of cusp forms). The local traces at residual cosets give rise to finite dimensional Hilbert algebras which we call "residual algebras". Their generic structure reduces (via parabolic induction) to the case of the residual algebras at "residual points" of certain semisimple subquotients of the Hecke algebra (see Subsection 4.3). 
These matters concerning the localization of $\mathcal{H}$ are studied in Section 4, leading to the main result Theorem 4.43. The support of the Plancherel measure and the Plancherel density are expressed in terms of the discrete series of Levi subquotient algebras of $\mathcal{H}$, and their Plancherel masses (formal dimensions).

(4). At this point, two essential problems remain: The classification of the discrete series representations, and the determination of their formal dimensions. Regarding the first problem, we have determined the orbits $W_{0} r \in W_{0} \backslash T$ which arise as the central character of a discrete series representation of $\mathcal{H}$ in Theorem 3.29. We have no further information to offer on this problem in this paper.

Section 5 is devoted to the second of these problems. In order to explain our approach, let $\nu\left(\left\{W_{0} r\right\}\right)$ denote the Plancherel mass of the central character $W_{0} r$ with respect to the restriction of the tracial state $\tau$ to the center $\mathcal{Z}$ of $\mathcal{H}$. In Subsection 3.4 we find that the formal dimension of an irreducible discrete series representation $\delta$ whose central character is a certain residual point $W_{0} r$, is equal to the product of $\nu\left(\left\{W_{0} r\right\}\right)$ and a certain positive real number $d_{\delta}>0$ (called "the residual degree" of $\delta$ ) depending on $\delta$ (see Corollary 3.32 and Theorem 3.25). (These residual degrees are normalized such that $\sum \operatorname{dim}(\delta) d_{\delta}=1$, where the sum runs over all square integrable $\delta$ whose central character is $W_{0} r$ ).

The factor $\nu\left(\left\{W_{0} r\right\}\right)$ is a certain explicit product (explicit up to a nonzero rational multiple) of rational functions evaluated at the central character $W_{0} r$. The problem that arises here is that we have not much information about the behaviour of the individual "residual degrees" $d_{\delta}$ as functions of the parameters $q(s)$.

In Section 5 we resolve this matter. If we write the labels $q(s)>0$ in the form $q(s)=\mathbf{q}^{f_{s}}$ for certain real numbers $f_{s}$ and $\mathbf{q}>1$, we prove that the residual Hilbert algebras are independent of the base $\mathbf{q}>1$. In other words, the constants $d_{\delta}>0$ are independent of $\mathbf{q}>1$. This proves that all irreducible discrete series representations of $\mathcal{H}$ associated with a central character $W_{0} r$, have a formal dimension which is proportional to the mass $\nu\left(\left\{W_{0} r\right\}\right)$, with a positive real ratio of proportionality which is independent of $\mathbf{q}>1$.

In addition we conjecture that the positive reals $d_{\delta}$ are actually rational numbers (cf. Conjecture 2.27). This conjecture is subject of joint work in progress with Mark Reeder and Antony Wasserman.

(5). In Appendix 7 we study the geometry of the set of singularities with maximal pole order of the rational $n$-form

$$
\frac{d t}{c(t) c\left(t^{-1}\right)}
$$


on $T$. This leads to the notion "residual coset", which is crucially important for the understanding of the residues of the kernel for $\tau$ in (3.1). It is analogous to the notion of residual subspace which was introduced in [18]. The collection of these cosets can be classified, and from this classification we verify certain important geometric properties of this collection. These geometric facts are used in Section 3 (especially in Subsection 3.4) to establish regularity properties of the residues to be considered in this paper.

1.0.3. Residue calculus. Let us make some remarks about the "residue calculus" on which much of this paper is ultimately based. At the heart of it lies the elementary Lemma 3.4, which is an adapted version of Lemma 3.1 of [18]. This lemma roughly states that on a complex torus $T$, any linear functional $\tau$ on the ring of Laurent polynomials $\mathbb{C}[T]$ of the form

$$
\tau(f)=\int_{t_{0} T_{u}} f \omega
$$

where $\omega$ is a rational $(n, 0)$-form whose pole set is a union of cosets of codimension 1 subtori of $T$, can be represented by a unique collection of "local distributions" living on certain cosets of the compact form $T_{u}$ of $T$, and satisfying certain support conditions.

In the context of the representation theory of $\mathcal{H}$, this lemma becomes remarkably efficient. We apply the lemma to linear functionals of the form $a \rightarrow \tau(a h)$, where $h \in \mathcal{H}$ and $a \in \mathcal{A}$, a maximal abelian subalgebra of $\mathcal{H}$, using formula (3.1). At this stage we symmetrize the "local distributions" for the action of $W_{0}$. Using the elementary notion of "approximating sequence" (see Lemma 3.5) it is not hard to show that the symmetrized local distributions inherit the positivity of $\tau$. This implies easily that these symmetrized distributions are in fact compactly supported measures on the spectrum $W_{0} \backslash T$ of the center $\mathcal{Z}=\mathcal{A}^{W_{0}}$ of $\mathcal{H}$, with values in the positive traces on $\mathcal{H}$ (see Corollary 3.23). This means that all higher order terms in the local distributions cancel out by the symmetrization by $W_{0}$.

In addition it follows by positivity that all measures are absolutely continuous with respect to a scalar measure $\nu$, the Plancherel measure of the center $\mathcal{Z}$ of $\mathcal{H}$. In fact $\nu$ is obtained by evaluation of the symmetrized local distributions at $1 \in \mathcal{H}$. Fortunately the poles of formula (3.1) simplify to the poles of expression (1.2) by this evaluation. In this way we see that the contributions at non-residual, quasi-residual cosets must cancel. We can bring into play the geometric properties of the residual subspaces now, established in Appendix 7, to prove that 
the Plancherel measure is smooth on its support, that the local traces are tempered, and that the local traces at discrete mass points of $\nu$ are finite linear combinations of discrete series characters.

We get in this way a decomposition of $\tau$ as a superposition of positive "local traces", which is an important step towards the Plancherel decomposition of $\mathcal{H}$.

At the time of the writing of the paper [18], working on the quantum theory of a certain exactly solvable $n$-particle systems, we were not aware of the already existing results in the spirit of the above lemma on existence and uniqueness of residue distributions. But we should certainly mention here the basic work of Langlands [24], where residues of Eisenstein series are studied in the theory of automorphic forms for reductive groupes. We also mention the work of Arthur [1], [3] in this direction. Langlands' work [24] was elucidated by Moeglin and Waldspurger in [35]. Langlands' result on existence of "residue data" can be found in Theorem V.2.2, and on uniqueness of "residue data" in the formulation of Theorem V.3.13(i) of [35]. It should be pointed out however that these results are of a different nature than our basic Lemma 3.4. Lemma 3.4 is a (very elementary) general result in distribution theory, which has nothing to do with group theory. On the other hand, the above results in [35] are formulated with already symmetrized "residue data", using intertwining operators. In order to even formulate a uniqueness property in this setting, one first needs to show rather deep statements on the holomorphic continuation of certain residue sums of Eisenstein series (see V.3.2 of [35]).

More recently, inspired by the approach in [18], Van den Ban and Schlichtkrull [5] extended the method by allowing for so-called residue weights. In this generality they applied the residue calculus in their proof of the Plancherel formula for semisimple symmetric spaces.

\section{Preliminaries and description of results}

The algebraic background for our analysis was discussed in the paper [37]. The main result of that paper is an inversion formula (see equation (3.1)) which will be the starting point in this paper. The purpose of this section is to define the affine Hecke algebra $\mathcal{H}$ and to review the relevant notations and concepts involved in the above result. Moreover we introduce a $C^{*}$-algebra hull $\mathfrak{C}$ of $\mathcal{H}$, which will be the main object of study in this paper. Finally we will give a more precise outline of the results in the paper. We refer the reader to [26] and [37] for a more systematic introduction of the basic algebraic notions. 


\subsection{The affine Weyl group and its root datum}

A reduced root datum is a 5 -tuple $\mathcal{R}=\left(X, Y, R_{0}, R_{0}^{\vee}, F_{0}\right)$, where $X$ and $Y$ are free abelian groups with perfect pairing over $\mathbb{Z}, R_{0} \subset X$ is a reduced integral root system, $R_{0}^{\vee} \subset Y$ is the dual root system of coroots of $R_{0}$, and $F_{0} \subset R_{0}$ is a basis of simple roots. Each element $\alpha \in R_{0}$ determines a reflection $s_{\alpha} \in \mathrm{GL}(X)$ by

$$
s_{\alpha}(x)=x-x\left(\alpha^{\vee}\right) \alpha .
$$

The group $W_{0}$ in $\mathrm{GL}(X)$ generated by the $s_{\alpha}$ is called the Weyl group. As is well known, this group is in fact generated by the set $S_{0}$ consisting of the reflections $s_{\alpha}$ with $\alpha \in F_{0}$. The set $S_{0}$ is called the set of simple reflections in $W_{0}$.

By definition the affine Weyl group $W$ associated with a reduced root datum $\mathcal{R}$ is the group $W=W_{0} \ltimes X$. This group $W$ naturally acts on the set $X$.

We choose once and for all a rational, symmetric, positive definite, $W_{0}$-invariant pairing $\langle\cdot, \cdot\rangle$ on $\mathbb{Q} \otimes Y$. This defines a $W_{0}$ pairing on the Euclidean spaces $\mathfrak{t}:=\mathbb{R} \otimes Y$ and its dual $\mathfrak{t}^{*}=\mathbb{R} \otimes Y$. The action of $W$ on $X$ extends to an action of $W$ on $\mathfrak{t}^{*}$ by means of isometries.

We can identify the set of integral affine linear functions on $X$ with $Y \times \mathbb{Z}$ via $(y, k)(x):=(x, y)+k$. It is clear that $w \cdot f(x):=f\left(w^{-1} x\right)$ defines an action of $W$ on $Y \times \mathbb{Z}$. The affine root system is by definition the subset $R^{\text {aff }}=R_{0}^{\vee} \times \mathbb{Z} \subset Y \times \mathbb{Z}$. Notice that $R^{\text {aff }}$ is a $W$-invariant set in $Y \times \mathbb{Z}$ containing the set of coroots $R_{0}^{\vee}$. Every element $a=$ $\left(\alpha^{\vee}, k\right) \in R^{\text {aff }}$ defines an affine reflection $s_{a} \in W$, acting on $X$ by

$$
s_{a}(x)=x-a(x) \alpha .
$$

The reflections $s_{a}$ with $a \in R^{\text {aff }}$ generate a normal subgroup $W^{\text {aff }}=$ $W_{0} \ltimes Q$ of $W$, where $Q \subset X$ denotes the root lattice $Q=\mathbb{Z} R_{0}$. We can choose a basis of simple affine roots $F^{\text {aff }}$ by

$$
F^{\mathrm{aff}}:=\left\{\left(\alpha^{\vee}, 1\right) \mid \alpha \in S^{m}\right\} \cup\left\{\left(\alpha^{\vee}, 0\right) \mid \alpha \in F_{0}\right\},
$$

where $S^{m}$ consists of the set of minimal coroots with respect to the dominance ordering on $Y$. It is easy to see that every affine root is an integral linear combination of elements from $F^{\text {aff }}$ with either all nonnegative or all nonpositive coefficients. The set $R^{\text {aff }}$ of affine roots is thus a disjoint union of the set of positive affine roots $R_{+}^{\text {aff }}$ and the set of negative affine roots $R_{-}^{\text {aff }}$. The set $S^{\text {aff }}$ of simple reflections in $W$ is by definition the set of reflections in $W$ associated with the fundamental affine roots. They constitute a set of Coxeter generators for the normal subgroup $W^{\text {aff }} \subset W$. 
There exists an Abelian complement to $W^{\text {aff }}$ in $W$. This is best understood by introducing the important length function $l$ on $W$. The splitting $R^{\text {aff }}=R_{+}^{\text {aff }} \cup R_{-}^{\text {aff }}$ described above implies that $R_{+}^{\text {aff }} \cap s_{a}\left(R_{-}^{\text {aff }}\right)=$ $\{a\}$ when $a \in F^{\text {aff }}$. Define, as usual, the length of an element $w \in W$ by

$$
l(w):=\left|R_{+}^{\mathrm{aff}} \cap w^{-1}\left(R_{-}^{\mathrm{aff}}\right)\right| .
$$

It follows that, when $a \in F^{\text {aff }}$,

$$
l\left(s_{a} w\right)=\left\{\begin{array}{l}
l(w)+1 \text { if } w^{-1}(a) \in R_{+}^{\mathrm{aff}} . \\
l(w)-1 \text { if } w^{-1}(a) \in R_{-}^{\mathrm{aff}} .
\end{array}\right.
$$

For any $w \in W$ we may therefore write $w=\omega \tilde{w}$ with $\tilde{w} \in W^{\text {aff }}$ and with $l(\omega)=0$ (or equivalently, $\omega\left(F^{\text {aff }}\right)=F^{\text {aff }}$ ). This shows that the set $\Omega$ of elements of length 0 is a subgroup of $W$ which is complementary to the normal subgroup $W^{\text {aff }}$, so that we have the decomposition

$$
W=\Omega \ltimes W^{\mathrm{aff}} .
$$

Hence $\Omega \simeq W / W^{\text {aff }} \simeq X / Q$ is a finitely generated Abelian group.

Let $m: X \rightarrow P$ (where $P$ denotes the weight lattice) denote the homomorphism that is adjoint to the inclusion $Q^{\vee} \rightarrow Y$. If we write $Z_{X} \subset X$ for its kernel, then $Z_{X} \subset \Omega$. We have $\Omega / Z_{X}=\Omega_{f}$ where $Z_{X}$ is free and $\Omega_{f}=m(X) / Q \subset P / Q$ is finite. It is easy to see that $Z_{X}$ is the subgroup of elements in $X$ that are central in $W$. The finite group $\Omega_{f}$ acts faithfully on $S^{\text {aff }}$ by diagram automorphisms.

The dual cone $X^{+}$of the cone $Q_{+}$spanned by the positive roots is called the cone of dominant elements of $X$. Thus $x \in X$ belongs to $X^{+}$ if and only if $\left\langle x, \alpha^{\vee}\right\rangle \geq 0$ for all positive roots $\alpha \in R_{0,+}$. Notice that $X^{+} \cap X^{-}$equals the sublattice $Z_{X} \subset X$ of translations of length 0 .

Write $v=v_{0}+v^{0}$ for the splitting of $v \in \mathfrak{t}^{*}$ according to the orthogonal decomposition $\mathfrak{t}^{*}=\mathfrak{t}^{*}{ }_{0}+\mathfrak{t}^{* 0}$, where $\mathfrak{t}^{*}{ }_{0}=\mathbb{R} \otimes Q$. We define a norm

$$
\mathcal{N}(w)=l(w)+\left\|w(0)^{0}\right\|
$$

for $w \in W$. Notice that for all $w, w^{\prime} \in W, w w^{\prime}(0)^{0}=w(0)^{0}+w^{\prime}(0)^{0}$. Thus $\mathcal{N}(\omega w)=\mathcal{N}(w \omega)=l(w)+\mathcal{N}(\omega)$ if $w \in W^{\text {aff }}$ and $\omega \in \Omega$. We also see that for all $\omega \in \Omega, \mathcal{N}\left(\omega^{k}\right)=k \mathcal{N}(\omega)$ for $k \in \mathbb{N}$. It follows easily that $\omega \in \Omega$ has finite order if and only if $\mathcal{N}(\omega)=0$. Finally notice that it also follows that

$$
\mathcal{N}\left(w w^{\prime}\right) \leq \mathcal{N}(w)+\mathcal{N}\left(w^{\prime}\right)
$$


ON THE SPECTRAL DECOMPOSITION OF AFFINE HECKE ALGEBRAS 11

\subsection{Parabolic subsystems}

An important role will be played by parabolic subgroups of a Weyl group. A root subsystem $R^{\prime} \subset R_{0}$ is called parabolic if $R^{\prime}=R_{0} \cap \mathbb{Q} R^{\prime}$. Let $P \subset R_{0,+} \cap R^{\prime}$ be the basis of simple roots. We then often write $R_{P}$ instead of $R^{\prime}$. The subgroup $W_{P}:=W\left(R_{P}\right)$ is called the associated parabolic subgroup. If $P \subset F_{0}$, we call $R_{P}$ and $W_{P}$ standard parabolic. Every parabolic subgroup is conjugate to a standard parabolic subgroup. We denote by $W^{P}$ the set of left cosets $W_{0} / W_{P}$. If $W_{P}$ is standard, we identify this quotient with the set of distinguished coset representatives of minimal length.

In many instances we obtain a parabolic subsystem $R^{\prime}$ as the set of roots orthogonal to some subspace $V^{L} \subset \mathfrak{t}:=\mathbb{R} \otimes Y$ which has the property that $V^{L}=\cap \operatorname{ker}(\alpha)$ where we take the intersection over all the roots $\alpha$ such that $\alpha\left(V^{L}\right)=0$. By abuse of notation we usually denote this parabolic subsystem by $R_{L}$. Similarly we write $W_{L}$ and $W^{L}$. We now denote the basis of $R_{L,+}$ by $F_{L}$.

To a parabolic subsystem $R_{P} \subset R_{0}$ we associate a root datum $\mathcal{R}^{P}:=$ $\left(X, Y, R_{P}, R_{P}^{\vee}, P\right)$ and a root datum $\mathcal{R}_{P}:=\left(X_{P}, Y_{P}, R_{P}, R_{P}^{\vee}, P\right)$ where $Y_{P}:=Y \cap \mathbb{Q} R_{P}^{\vee}$ and $X_{P}:=X /\left(X \cap\left(R_{P}^{\vee}\right)^{\perp}\right)$.

\subsection{Root labels}

The second ingredient in the definition of $\mathcal{H}$ is a function $q$ on $S^{\text {aff }}$ with values in the group of invertible elements of a commutative ring, such that

$$
q(s)=q\left(s^{\prime}\right) \text { if } s \text { and } s^{\prime} \text { are conjugate in } W .
$$

A function $q$ on $S^{\text {aff }}$, satisfying 2.7 , can clearly be extended uniquely to a length-multiplicative function on $W$, also denoted by $q$. By this we mean that the extension satisfies

$$
q\left(w w^{\prime}\right)=q(w) q\left(w^{\prime}\right)
$$

whenever

$$
l\left(w w^{\prime}\right)=l(w)+l\left(w^{\prime}\right)
$$

and in addition,

$$
\forall \omega \in \Omega: q(\omega)=1
$$

Conversely, every length multiplicative function on $W$ restricts to a

function on $S^{\text {aff }}$ that satisfies 2.7. Another way to capture the same 
information is by assigning labels $q_{a}$ to the affine roots $a \in R^{\text {aff }}$. These labels are uniquely determined by the rules

(i) $q_{w a}=q_{a} \forall w \in W$, and

(ii) $q\left(s_{a}\right)=q_{a+1} \forall a \in F^{\mathrm{aff}}$.

Note that a translation $t_{x}$ acts on an affine root $a=\left(\alpha^{\vee}, k\right)$ by $t_{x} a=$ $a-\alpha^{\vee}(x)$. Hence by $(i), q_{a}=q_{\alpha^{\vee}}$, except when $\alpha^{\vee} \in 2 Y$, in which case $q_{a}=q_{\left(\alpha^{\vee}, k(\bmod 2)\right)}$. This last case occurs iff $W$ contains direct factors which are isomorphic to the affine Coxeter group whose diagram equals $C_{n}^{\text {aff }}$.

Yet another manner of labeling will play an important role. It involves a possibly non-reduced root system $R_{\mathrm{nr}}$, which is defined by:

$$
R_{\mathrm{nr}}:=R_{0} \cup\left\{2 \alpha \mid \alpha^{\vee} \in R_{0}^{\vee} \cap 2 Y\right\} .
$$

Now define labels for the roots $\alpha^{\vee} / 2$ in $R_{\mathrm{nr}}^{\vee} \backslash R_{0}^{\vee}$ by:

$$
q_{\alpha^{\vee} / 2}:=\frac{q_{1+\alpha^{\vee}}}{q_{\alpha \vee}}
$$

This choice is natural, because it implies the formula

$$
q(w)=\prod_{\alpha \in R_{\mathrm{nr},+} \cap w^{-1} R_{\mathrm{nr},-}} q_{\alpha \vee}
$$

for all $w \in W_{0}$.

Let $R_{L} \subset R_{0}$ be a parabolic root subsystem. With respect to the root datum $\mathcal{R}_{L}$ we have $R_{L, \mathrm{nr}}=\mathbb{Q} R_{L} \cap R_{\mathrm{nr}} \subset R_{\mathrm{nr}}$. In this sense we can define a label function denoted by $q_{L}$ for the root datum $\mathcal{R}_{L}$, by restriction from $R_{\mathrm{nr}}^{\vee}$ to $R_{L, \mathrm{nr}}^{\vee}$. Similarly, we define $q^{L}$ by restriction of $q$ to $\mathcal{R}^{L}$

We denote by $R_{1}$ the root system of long roots in $R_{\mathrm{nr}}$. In other words

$$
R_{1}:=\left\{\alpha \in R_{\mathrm{nr}} \mid 2 \alpha \notin R_{\mathrm{nr}}\right\} .
$$

\subsection{The Iwahori-Hecke algebra as a Hilbert algebra}

Many of the results of this subsection are well known, see [34]. Let $\mathcal{R}$ be a root datum, and let $\mathbf{q}$ be a real number with $\mathbf{q}>1$. We assume that for all $s \in S^{\text {aff }}$ we are given a real number $f_{s}$. Throughout this paper we use the convention that the labels as discussed in the previous subsection are defined by:

Convention 2.1. The labels are of the form

$$
q(s)=\mathbf{q}^{f_{s}} \forall s \in S^{\mathrm{aff}} .
$$


We write $q:=(q(s))_{s \in S_{\text {aff }}}$ for the corresponding label function on $S^{\text {aff }}$. The following theorem is well known.

Theorem 2.2. There exists a unique complex associative algebra $\mathcal{H}=$ $\mathcal{H}(\mathcal{R}, q)$ with $\mathbb{C}$-basis $\left(T_{w}\right)_{w \in W}$ which satisfy the following relations:

(a) If $l\left(w w^{\prime}\right)=l(w)+l\left(w^{\prime}\right)$ then $T_{w} T_{w^{\prime}}=T_{w w^{\prime}}$.

(b) If $s \in S^{\text {aff }}$ then $\left(T_{s}+1\right)\left(T_{s}-q(s)\right)=0$.

The algebra $\mathcal{H}=\mathcal{H}(\mathcal{R}, q)$ is called the affine Hecke algebra (or IwahoriHecke algebra) associated to $(\mathcal{R}, q)$.

We equip the Hecke algebra $\mathcal{H}$ with an anti-linear anti-involutive $*$ operator defined by

$$
T_{w}^{*}=T_{w^{-1}}
$$

In addition, we define a trace functional $\tau$ on $\mathcal{H}$, by means of $\tau\left(T_{w}\right)=$ $\delta_{w, e}$. It is a well known basic fact that

$$
\tau\left(T_{w}^{*} T_{w^{\prime}}\right)=\delta_{w, w^{\prime}} q(w),
$$

implying that $\tau$ is positive and central. Hence the formula

$$
\left(h_{1}, h_{2}\right):=\tau\left(h_{1}^{*} h_{2}\right),
$$

defines an Hermitian inner product satisfying the following rules:

$$
\begin{aligned}
& \text { (i) }\left(h_{1}, h_{2}\right)=\left(h_{2}^{*}, h_{1}^{*}\right) . \\
& \text { (ii) }\left(h_{1} h_{2}, h_{3}\right)=\left(h_{2}, h_{1}^{*} h_{3}\right) .
\end{aligned}
$$

The basis $T_{w}$ is orthogonal for $(\cdot, \cdot)$. We put

$$
N_{w}:=q(w)^{-1 / 2} T_{w}
$$

for the orthonormal basis of $\mathcal{H}$ that is obtained from the orthogonal basis $T_{w}$ by scaling. Let us denote by $\lambda(h)$ and $\rho(h)$ the left and right multiplication operators on $\mathcal{H}$ by an element $h \in \mathcal{H}$. Let $\mathfrak{H}$ be the Hilbert space obtained from $\mathcal{H}$ by completion; in other words, $\mathfrak{H}$ is the Hilbert space with Hilbert basis $N_{w}$. The operator $*$ extends to an isometric involution on $\mathfrak{H}$. Let $B(\mathfrak{H})$ denote the space of bounded operators on the Hilbert space $\mathfrak{H}$.

Lemma 2.3. For all $h \in \mathcal{H}$, both $\lambda(h)$ and $\rho(h)$ extend to $\mathfrak{H}$ as bounded operators (elements of $B(\mathfrak{H})$ ), with $\|\lambda(h)\|=\|\rho(h)\|$. For a simple reflection $s \in S^{\text {aff }},\left\|\lambda\left(N_{s}\right)\right\|=\max \left\{q(s)^{ \pm 1 / 2}\right\}$.

Proof. We first prove the formula for the norm of $\left\|\lambda\left(N_{s}\right)\right\|\left(s \in S^{\text {aff }}\right)$. For every $w$ such that $l(s w)>l(w), \lambda\left(N_{s}\right)$ acts on the two-dimensional subspace $V_{w}$ of $\mathcal{H}$ spanned by $N_{w}$ and $N_{s w}$ as a self-adjoint operator with eigenvalues $q(s)^{1 / 2}$ and $-q(s)^{-1 / 2}$. Since $\mathfrak{H}$ is the Hilbert sum of the subspaces $V_{w}$, we see that $\lambda\left(N_{s}\right)$ extends to $\mathfrak{H}$ as a self-adjoint 
operator with operator norm equal to $q(s)^{ \pm 1 / 2}$. Hence for any $h \in$ $\mathcal{H}, \lambda(h)$ extends as a bounded operator on $\mathfrak{H}$. Finally notice that $\left(\lambda(h)^{*}(x)\right)^{*}=\rho(h)(x)$, proving the equality $\|\lambda(h)\|=\|\rho(h)\|$.

The above lemma shows that $\mathcal{H}$ has the structure of a Hilbert algebra in the sense of Dixmier [14]. Moreover, this Hilbert algebra is unital, and the Hermitian product is defined with respect to the trace $\tau$.

We define the operator norm $\|\cdot\|_{o}$ on $\mathcal{H}$ by $\|h\|_{o}:=\|\lambda(h)\|=\|\rho(h)\|$. The closure of $\mathcal{H}$ with respect to the operator norm $\|\cdot\|_{o}$ is denoted by $\mathfrak{C}$. The map $\lambda(\rho)$ extends to an isometry from $\mathfrak{C}$ to the $C^{*}$-subalgebra $\lambda(\mathfrak{C}) \subset B(\mathfrak{H})(\rho(\mathfrak{C}) \subset B(\mathfrak{H})$ resp. $)$, the norm closure of $\lambda(\mathcal{H})(\rho(\mathcal{H})$ resp.).

We identify $\mathfrak{C}$ with a subset of $\mathfrak{H}$ via the continuous injection $c \rightarrow$ $\lambda(c)(1)$. We equip $\mathfrak{C}$ with the structure of a unital $C^{*}$-algebra by the product $c_{1} c_{2}:=\lambda\left(c_{1}\right)\left(c_{2}\right)=\rho\left(c_{2}\right)\left(c_{1}\right)$ and the $*$-operator coming from $\mathfrak{H}$. Then $\lambda(\rho)$ is a faithful left (right) representation of $\mathfrak{C}$ in the Hilbert space $\mathfrak{H}$ (note that we consider $\rho$ as a right representation on $\mathfrak{H}$ ).

Definition 2.4. We call $\mathfrak{C}$ the reduced $C^{*}$-algebra of $\mathcal{H}$, and $\lambda(\rho)$ is called the left (right) regular representation of $\mathfrak{C}$ on $\mathfrak{H}$.

An element $a \in \mathfrak{H}$ is called bounded if there exists an element $\lambda(a) \in$ $B(\mathfrak{H})$ such that for all $h \in \mathcal{H}$,

$$
\lambda(a)(h)=\rho(h)(a) .
$$

By continuity we see that $\lambda(a)$ is uniquely determined by $a=\lambda(a)(1)$.

When $a \in \mathfrak{H}$ is bounded, there also exists a unique $\rho(a)$ such that for all $h \in \mathcal{H}$,

$$
\rho(a)(h)=\lambda(h)(a) .
$$

It is obvious that the elements of $\mathfrak{C}$ are bounded. Let us denote by $\mathfrak{N} \subset$ $\mathfrak{H}$ the subspace of bounded elements. We equip $\mathfrak{N}$ with the involutive algebra structure defined by the product $n_{1} n_{2}:=\lambda\left(n_{1}\right)\left(n_{2}\right)=\rho\left(n_{2}\right)\left(n_{1}\right)$ and the $*$ operator as before.

Proposition 2.5. The subspace $\lambda(\mathfrak{N}):=\{\lambda(a) \mid a \in \mathfrak{N}\} \subset B(\mathfrak{H})$ is the von Neumann algebra completion of $\lambda(\mathcal{H})$. In other words, $\lambda(\mathfrak{N})$ is the closure of $\lambda(\mathcal{H})$ in $B(\mathfrak{H})$ with respect to the strong topology (defined by the semi-norms $T \rightarrow\|T(x)\|$ with $x \in \mathfrak{H})$. The analogous statements hold when we replace $\lambda$ by $\rho$. The centralizing algebra of $\lambda(\mathfrak{N})$ is $\rho(\mathfrak{N})$.

Proof. All this can be found in [14], Chapitre I, paragraphe 5. In general, $\lambda(\mathfrak{N})$ is a two-sided ideal of the von Neumann algebra hull of $\lambda(\mathcal{H})$, but in the presence of the unit $1 \in \mathcal{H}$ the two spaces coincide. 
ON THE SPECTRAL DECOMPOSITION OF AFFINE HECKE ALGEBRAS 15

In fact, when $A \in B(\mathfrak{H})$ and $A$ is in the strong closure of $\lambda(\mathcal{H})$, it is simple to see that $A(1) \in \mathfrak{H}$ is bounded.

The pre-Hilbert structure coming from $\mathfrak{H}$ gives $\mathfrak{N}$ itself the structure of a unital Hilbert algebra. The algebra $\mathfrak{N}$ can and will be identified with its associated standard von Neumann algebra $\lambda(\mathfrak{N})$. In this situation, $\mathfrak{N}$ is said to be a saturated Hilbert algebra (with unit element).

Let $\mathcal{H}^{*}$ denote the algebraic dual of $\mathcal{H}$, equipped with its weak topology. Notice that $\tau$ extends to $\mathcal{H}^{*}$ by the formula $\tau(\phi):=\phi(1)$. The *-operator can be extended to $\mathcal{H}^{*}$ by $\phi^{*}(h):=\overline{\phi\left(h^{*}\right)}$. We have the following chain of inclusions:

$$
\mathcal{H} \subset \mathfrak{C} \subset \mathfrak{N} \subset \mathfrak{H} \subset \mathfrak{C}^{\prime \prime} \subset \mathcal{H}^{*}
$$

(where $\mathfrak{C}^{\prime}$ denotes the space of continuous linear functionals on $\mathfrak{C}$ ).

Proposition 2.6. The restriction of $\tau$ to $\mathfrak{N}$ is central, positive and finite. It is the natural trace of the Hilbert algebra $\mathfrak{N}$, in the sense that

$$
\tau(a)=(b, b)
$$

for every positive $a \in \mathfrak{N}$, and $b \in \mathfrak{N}$ such that $a=b^{2}$.

Proof. A square root $b$ is in $\mathfrak{N}$ and is Hermitian (i.e. $b^{*}=b$ ). Then $(b, b)=(1, a)=\tau(a)$.

Corollary 2.7. The Hilbert algebra $\mathfrak{N}$ is finite.

Corollary 2.8. The tracial state $\tau$ on $\mathfrak{C}$ is finite, and we have $\lambda=\lambda_{\tau}$ and $\rho=\rho_{\tau}$, where $\lambda_{\tau}$ and $\rho_{\tau}$ are the representations of $\mathfrak{C}$ naturally associated with the state $\tau$ (the classical GNS-construction).

Proof. This is immediate from the definitions, see [15], Paragraphe 6.7.

\subsection{Bernstein's description of the center $\mathcal{Z}$}

By a well known (unpublished) result of J. Bernstein (see [26]), $\mathcal{H}$ can be viewed as the product $\mathcal{H}_{0} \mathcal{A}$ or $\mathcal{A H}_{0}$ of an abelian subalgebra $\mathcal{A}$ (isomorphic to the group algebra of the lattice $X$ ), and the Hecke algebra $\mathcal{H}_{0}=\mathcal{H}\left(W_{0},\left.q\right|_{S_{0}}\right)$ of the finite Weyl group $W_{0}$. Both product decompositions $\mathcal{H}_{0} \mathcal{A}$ and $\mathcal{A} \mathcal{H}_{0}$ give a linear isomorphism of $\mathcal{H}$ with the tensor product $\mathcal{H}_{0} \otimes \mathcal{A}$. The relations between products in $\mathcal{H}_{0} \mathcal{A}$ and in $\mathcal{A H}_{0}$ are described by the Bernstein-Zelevinski-Lusztig relations (see for example [37], Theorem 1.10), and with the above additional description of the structure of $\mathcal{A}$ and $\mathcal{H}_{0}$ these give a complete presentation of $\mathcal{H}$. 
The algebra $\mathcal{A}$ has a $\mathbb{C}$-basis of invertible elements $\theta_{x}$ (with $x \in X$ ) such that $x \rightarrow \theta_{x}$ is a monomorphism of $X$ into the group of invertible elements of $\mathcal{H}$. This monomorphism is uniquely determined by the property that $\theta_{x}=N_{t_{x}}$ (see (2.17)) when $x \in X^{+}$. As an important corollary of this presentation of $\mathcal{H}$, Bernstein identified the center $\mathcal{Z}$ of $\mathcal{H}$ as the space $\mathcal{Z}=\mathcal{A}^{W_{0}}$ of $W_{0}$-invariant elements in $\mathcal{A}$ (see [37], Theorem 1.11). The following Proposition is well known and easy (cf. [37], Proposition 1.12):

Proposition 2.9. Let $w_{0} \in W_{0}$ denote the longest element of $W_{0}$. Then we have for all $x \in X$ :

$$
\theta_{x}^{*}=T_{w_{0}} \theta_{-w_{0}(x)} T_{w_{0}}^{-1} .
$$

In particular, $\mathcal{A} \subset \mathcal{H}$ is not a*-subalgebra in general. The center $\mathcal{Z} \subset \mathcal{H}$ is a Hilbert subalgebra.

Let $T=\operatorname{Spec}(\mathcal{A})=\operatorname{Hom}_{\mathbb{Z}}\left(X, \mathbb{C}^{\times}\right)$. This algebraic torus of complex characters of $X$ has a natural $W_{0}$-action, and we have $\operatorname{Spec}(\mathcal{Z}) \simeq$ $W_{0} \backslash T$.

Proposition 2.10. The Hecke algebra $\mathcal{H}$ is finitely generated over its center $\mathcal{Z}$. At a maximal ideal $m=m_{t}$ (with $t \in T$ ) of $\mathcal{Z}$, the local rank equals $\left|W_{0}\right|^{2}$ if and only if the stabilizer group $W_{t} \subset W_{0}$ is generated by reflections.

Proof. It is clear that $\mathcal{H} \simeq \mathcal{H}_{0} \otimes \mathcal{A}$ is finitely generated over $\mathcal{Z}=\mathcal{A}^{W_{0}}$. When $W_{t}$ is generated by reflections, it is easy to see that the rank of $m$-adic completion $\hat{\mathcal{A}}_{m}$ over $\hat{\mathcal{Z}}_{m}$ is exactly $\left|W_{0}\right|$ (see Proposition 2.23(4) of $[37])$.

This fact plays a predominant role in the representation theory of $\mathfrak{C}$. Let us look at some basic consequences.

Corollary 2.11. $\quad$ (i) Let $\pi$ be a finite dimensional irreducible representation of $\mathcal{H}$ with representation space $V$. The dimension of $V$ is less than or equal to $\left|W_{0}\right|$.

(ii) In addition, the center $\mathcal{Z}$ of $\mathcal{H}$ acts by scalars on $V$. Thus $\pi$ determines a "central character" $t_{\pi} \in \operatorname{Spec}(\mathcal{Z})$ such that for all $z \in \mathcal{Z}, \pi(z)=t_{\pi}(z) \operatorname{Id}_{V}$.

(iii) The characters of any finite set of inequivalent finite dimensional irreducible representations of $\mathcal{H}$ are linearly independent.

(iv) A topologically irreducible *-representation $\pi$ of the involutive algebra $\mathcal{H}$ is finite dimensional. 
Proof. Elementary and well known. Use the Frobenius-Schur theorem for (iii), Dixmier's version of Schur's lemma for (iv), and Proposition 2.10 .

Corollary 2.12. (See also [34]) Restriction to $\mathcal{H}$ induces an injection of the set $\hat{\mathfrak{C}}$ into the space $\hat{\mathcal{H}}$ of finite dimensional irreducible *-representations of $\mathcal{H}$. Consequently, the $C^{*}$-algebra $\mathfrak{C}$ is of finite type $I$.

Proof. Because $\mathcal{H} \subset \mathfrak{C}$ is dense, it is clear that a representation of $\mathfrak{C}$ is determined by its restriction to $\mathcal{H}$ and that (topological) irreducibility is preserved. Hence by the previous Corollary, all irreducible representations of $\mathfrak{C}$ have finite dimension.

We equip $T$ and $W_{0} \backslash T$ with the analytic topology. Given $\pi \in \hat{\mathfrak{C}}$ we denote by $W_{0} t_{\pi} \in W_{0} \backslash T$ the character of $\mathcal{Z}$ such that $\chi_{\pi}(z)=$ $\operatorname{dim}(\pi) z\left(t_{\pi}\right)$ (note that $\pi(\mathcal{Z})$ can not vanish identically since $1 \in \mathcal{Z}$ ).

By Proposition 2.9, the $*$-operator on $\mathcal{Z}$ is such that $z^{*}(t)=\overline{z\left(\overline{t^{-1}}\right)}$. When $\pi \in \hat{\mathfrak{C}}$, we have $\chi_{\pi}\left(x^{*}\right)=\overline{\chi_{\pi}(x)}$. It follows that $\overline{t_{\pi}^{-1}} \in W_{0} t_{\pi}$ for all $\pi \in \hat{\mathfrak{C}}$. Let us denote by $W_{0} \backslash T^{\text {herm }}$ the closed subset $\left\{W_{0} t \in\right.$ $\left.W_{0} \backslash T \mid \overline{t_{\pi}^{-1}} \in W_{0} t_{\pi}\right\}$ of $W_{0} \backslash T$.

Proposition 2.13. The map $p_{z}: \hat{\mathfrak{C}} \rightarrow W_{0} \backslash T$ defined by $p_{z}(\pi)=W_{0} t_{\pi}$ is continuous and finite. Its image $S=p_{z}(\hat{\mathfrak{C}}) \subset W_{0} \backslash T^{\text {herm }}$ is the spectrum $\hat{\overline{\mathcal{Z}}}$ of the closure $\overline{\mathcal{Z}}$ of $\mathcal{Z}$ in $\mathfrak{C}$. The map $p_{z}: \hat{\mathfrak{C}} \rightarrow S$ is closed.

Proof. It is clear that the image is in $W_{0} \backslash T^{\text {herm }}$ and that the map is finite (by Proposition 2.10). Since $W_{0} \backslash T$ is Hausdorff and $\hat{\mathfrak{C}}$ is compact, the map $p_{z}$ is closed if it is continuous.

So it remains to show that $p_{z}$ is continuous. The closure $\overline{\mathcal{Z}} \subset \mathfrak{C}$ is a unital commutative $C^{*}$-subalgebra of $\mathfrak{C}$. By the Gelfand transform it is isomorphic to the algebra of continuous functions $C(\hat{\overline{\mathcal{Z}}})$ on the compact Hausdorff space $\hat{\overline{\mathcal{Z}}}$. Denote by $\alpha$ the map $\alpha: \hat{\mathfrak{C}} \rightarrow \hat{\mathcal{Z}}$ defined by the condition $\chi_{\pi} \mid \overline{\mathcal{Z}}=\operatorname{dim}(\pi) \alpha(\pi)$. By Proposition 2.10.2 of [15], $\alpha$ is surjective. In other words, every primitive ideal $M$ of $\mathfrak{C}$ intersects $\overline{\mathcal{Z}}$ in a maximal ideal $m$ of $\overline{\mathcal{Z}}$, and all maximal ideals of $\overline{\mathcal{Z}}$ are of this form. The corresponding surjective map from the set $\operatorname{Prim}(\mathfrak{C})$ of primitive ideals of $\mathfrak{C}$ to the set of maximal ideals $\operatorname{Max}(\overline{\mathcal{Z}})$ is also denoted by $\alpha$. Next we claim that $\alpha$ is continuous. The topologies of $\hat{\mathfrak{C}}$ and $\hat{\mathcal{Z}}$ are defined by the Jacobson topologies on $\operatorname{Prim}(\mathfrak{C})$ and $\operatorname{Max}(\overline{\mathcal{Z}})$. This means that $U \subset \operatorname{Prim}(\mathfrak{C})$ is closed if and only if every $M \in \operatorname{Prim}(\mathfrak{C})$ which contains $I(U)=\cap_{u \in U} u$ is in $U$. Let $V \subset \operatorname{Max}(\overline{\mathcal{Z}})$ be closed, and 
put $U=\alpha^{-1}(V)$. By the surjectivity of $\alpha$ we have $I(U) \cap \overline{\mathcal{Z}}=I(V)$. Hence if $M \in \operatorname{Prim}(\mathfrak{C})$ contains $I(U)$, then $\alpha(M)=M \cap \overline{\mathcal{Z}}$ contains $I(V)$, implying that $\alpha(M) \in V$. Therefore $M \in U$, proving that $U$ is closed as desired.

Next, we consider the injective map $\beta: \hat{\bar{Z}} \rightarrow W_{0} \backslash T$ defined by restriction to $\mathcal{Z} \subset \overline{\mathcal{Z}}$. Its image $S \subset W_{0} \backslash T^{\text {herm }}$ is bounded because for every $z \in \mathcal{Z},\|z\|_{o}=\max _{\chi \in \hat{\bar{Z}}}|\chi(z)|=\max _{s \in S}|z(s)|$, showing that each $|z|$ with $z \in \mathcal{Z}$ has a maximum on $S$. Because $\bar{S} \subset W_{0} \backslash T^{\text {herm }}$, we see that $z^{*}(s)=\overline{z(s)}$ for each $z \in \mathcal{Z}$ and $s \in \bar{S}$. By the Stone-Weierstrass theorem, the restriction to $S$ of a continuous function $f \in C\left(W_{0} \backslash T\right)$ can be uniformly approximated by elements in $\mathcal{Z}$ considered as functions on $S$. In other words, there exists a $z \in \overline{\mathcal{Z}}$ such that $f(\beta(\chi))=\chi(z)$ for all $\chi \in \hat{\bar{Z}}$. Hence $f \circ \beta$ is continuous on $\hat{\bar{Z}}$ for all $f \in C\left(W_{0} \backslash T\right)$, showing that $\beta$ is continuous and $S=\bar{S}$. Since $\hat{\bar{Z}}$ is compact and $S$ is Hausdorff it follows that $\beta: \hat{\bar{Z}} \rightarrow S$ is a homeomorphism. The proposition now follows from the remark that $p_{z}=\beta \circ \alpha$.

\subsection{Positive elements and positive functionals}

Definition 2.14. We denote by $\mathcal{H}_{+}$the set of Hermitian elements $h \in \mathcal{H}$ such that $\forall x \in \mathcal{H}:(h x, x) \geq 0$. We call this the set of positive elements of $\mathcal{H}$.

By spectral theory in the Hilbert completion $\mathfrak{H} \supset \mathcal{H}$, this is equivalent to saying that $\lambda(h) \in B(\mathfrak{H})$ is Hermitian and has its spectrum in $\mathbb{R}_{\geq 0}$. Thus $\mathcal{H}_{+}$is the intersection of $\mathcal{H}$ with the usual positive cone $\mathfrak{C}_{+}$of the completion $\mathfrak{C}$. It is clear that for all $x \in \mathcal{H}, x^{*} x \in \mathcal{H}_{+}$but not every positive element is of this form. We write $\mathcal{H}^{\text {re }}$ for the real subspace of Hermitian (or real) elements, i.e. $h \in \mathcal{H}$ such that $h^{*}=h$.

Lemma 2.15. (i) If $z \in \mathcal{Z}_{+}, h \in \mathcal{H}_{+}$then $z h \in \mathcal{H}_{+}$.

(ii) If $h \in \mathcal{H}^{\text {re }}$ and $A \in \mathbb{R}_{+}$such that $A \geq\|h\|_{o}$, then $A .1+h \in$ $\mathcal{H}_{+}$.

Proof. A square root $\sqrt{z} \in \overline{\mathcal{Z}}$, the closure of $\mathcal{Z}$ in $\mathfrak{C}$, of $z$ has obviously the property that $\lambda(\sqrt{z})=\rho(\sqrt{z})$. Hence for every $x \in \mathcal{H}, h \in \mathcal{H}_{+}$:

$$
(z h x, x)=(h \lambda(\sqrt{z}) x, \lambda(\sqrt{z}) x) \geq 0 .
$$

The second assertion follows since $\operatorname{Spec}(\lambda(h)) \subset\left[-\|h\|_{o},\|h\|_{o}\right]$.

Definition 2.16. We call a linear functional $\chi \in \mathcal{H}^{*}$ positive if $\chi(x) \geq$ 0 for all $x \in \mathcal{H}_{+}$. 
ON THE SPECTRAL DECOMPOSITION OF AFFINE HECKE ALGEBRAS 19

Corollary 2.17. $\quad$ (i) A positive linear functional $\chi \in \mathcal{H}^{*}$ extends uniquely to a continuous functional $\chi \in \mathfrak{C}^{\prime}$ with norm $\|\chi\|=$ $\chi(1)$.

(ii) The character $\chi_{\pi}$ of an irreducible representation $\pi$ of $\mathfrak{C}$ is a positive functional $\chi_{\pi} \in \mathfrak{C}^{\prime}$.

(iii) An irreducible *-representation $\pi$ of $\mathcal{H}$ extends to $\mathfrak{C}$ if and only if its character is a positive functional.

Proof. (i). By the above Lemma 2.15, $|\chi(x)| \leq \chi(1)\|x\|_{o}$ for all Hermitian $x \in \mathcal{H}$. In addition, the bitrace $(x, y):=\chi\left(x^{*} y\right)$ is a positive semi-definite Hermitian form, and thus satisfies the Schwarz inequality. Hence for arbitrary $x \in \mathcal{H}$ we have $|\chi(x)|^{2} \leq \chi\left(x^{*} x\right) \chi(1) \leq$ $\chi(1)^{2}\left\|x^{*} x\right\|_{o}=\chi(1)^{2}\|x\|_{o}^{2}$, proving the continuity of $\chi$.

(ii). Because every irreducible representation $\pi$ of $\mathfrak{C}$ is finite dimensional (Corollary 2.12), it is clear that the character $\chi_{\pi}(x)$ is a well defined positive functional on $\mathfrak{C}$. It is continuous by (i).

(iii). If the character $\chi_{\pi}$ is positive, we have by (i) that $\chi_{\pi}$ extends to a finite continuous character of $\mathfrak{C}$. Because $\mathfrak{C}$ is of finite type I (and thus liminal), the standard construction in [15], paragraphe 6.7 shows that there is up to equivalence a unique irreducible representation $\tilde{\pi}$ of $\mathfrak{C}$ whose character is $\chi_{\pi}$. The converse statement follows by (ii).

Remark 2.18. In general not all irreducible *-representations of $\mathcal{H}$ extend to $\mathfrak{C}$. See for instance Corollary 6.4.

\subsection{Casselman's criteria}

For later use, we discuss in this subsection a suitable version of Casselman's criteria (see [11], Lemma 4.4.1) to decide whether a representation of $\mathcal{H}$ is tempered (see the definition below) or is a subrepresentation of $\mathfrak{H}$. See also [34].

Recall the norm function $\mathcal{N}$ on $W$ as was introduced in Section 2.

Definition 2.19. A functional $f \in \mathcal{H}^{*}$ is called tempered if there exists an $N \in \mathbb{N}$ and constant $C>0$ such that for all $w \in W$,

$$
\left|f\left(N_{w}\right)\right| \leq C(1+\mathcal{N}(w))^{N} .
$$

Here $N_{w}=q(w)^{-1 / 2} T_{w}$ are the orthonormal basis elements of $\mathcal{H}$ introduced in (2.17).

Let $(V, \pi)$ be a finite dimensional module over $\mathcal{H}$, and let $t \in T$. We define $V_{t}:=\left\{v \in V \mid \forall a \in \mathcal{A} \exists n \in \mathbb{N}:(a-a(t))^{n}(v)=0\right\}$. The nonzero subspaces of the form $V_{t} \subset V$ are called the generalized 
$\mathcal{A}$-weight spaces of $V$. We call the corresponding elements $t \in T$ the $\mathcal{A}$-weights of $V$.

Lemma 2.20. (Casselman's criterion for temperedness). The following statements are equivalent:

(i) All matrix coefficients of $\pi$ are tempered.

(ii) The character $\chi$ of $\pi$ is tempered.

(iii) The weights $t$ of the generalized $\mathcal{A}$-weight spaces of $V$ satisfy $|x(t)| \leq 1$, for all $x \in X^{+}$.

Proof. (i) $\Rightarrow$ (ii). This is trivial.

(ii) $\Rightarrow$ (iii). If there exists a weight $t$ of $V$ violating the condition, then there exists a $x \in X^{+}$such that $|x(t)|>1$. We may assume that $|x(t)| \geq\left|x\left(t^{\prime}\right)\right|$ for all weights $t^{\prime}$ of $V$. By Lemma 4.4.1 of [11], the function

$$
f_{x}(n)=|x(t)|^{-n} \sum_{t^{\prime}} \operatorname{dim}\left(V_{t^{\prime}}\right) x\left(t^{\prime}\right)^{n}=|x(t)|^{-n} \chi\left(\theta_{n x}\right)
$$

is not summable on $\mathbb{N}$. Hence for all $\epsilon>0, \chi\left(\theta_{n x}\right)$ can not be bounded by a constant times $|x(t)|^{n(1-\epsilon)}$. On the other hand, suppose that $\chi$ is tempered. Since $\theta_{n x}=N_{n x}$ and $\mathcal{N}(n x)=n \mathcal{N}(x)$, we obtain that $\chi\left(\theta_{n x}\right)$ is bounded by a polynomial in $n$, a contradiction.

(iii) $\Rightarrow$ (i) Recall that the elements $T_{u} \theta_{x} T_{v}$ with $x \in X^{+}, u, v \in W_{0}$ span the subspace of $\mathcal{H}$ with basis $N_{w}$, where $w$ runs over the double coset $W_{0} x W_{0} \subset W$ (see the proof of Lemma 3.1 of [37]). It is not difficult to see that in fact we can write, for $w=u x v \in W_{0} x W_{0}$ with $x \in X^{+}$,

$$
N_{w}=\sum_{v^{\prime}, u^{\prime} \in W_{0}} c_{w,\left(u^{\prime}, v^{\prime}\right)} T_{u^{\prime}} \theta_{x} T_{v^{\prime}}
$$

such that the coefficients $c_{u x v,\left(u^{\prime}, v^{\prime}\right)}$ and $c_{u y v,\left(u^{\prime}, v^{\prime}\right)}$ are equal if $x$ and $y$ belong to the same facet of the cone $X^{+}$. Moreover, by the length formula [37], equation 1.1, we have

$$
l(x)-\left|R_{0,+}\right| \leq l(u x v) \leq l(x)+\left|R_{0,+}\right|
$$

with $l(x)=x\left(2 \rho^{\vee}\right)$. Thus we also have

$$
\mathcal{N}(x)-\left|R_{0,+}\right| \leq \mathcal{N}(u x v) \leq \mathcal{N}(x)+\left|R_{0,+}\right| .
$$

It therefore suffices to show that the matrix entries of $\pi\left(\theta_{x}\right)$ with $x \in$ $X^{+}$are polynomially bounded in $\mathcal{N}(x)=x\left(2 \rho^{\vee}\right)+\left\|x^{0}\right\|$. Since $V$ is a direct sum of generalized $\mathcal{A}$-weight spaces $V_{t}$, it is enough to consider the matrix coefficients of a generalized $\mathcal{A}$-weight space $V_{t}$ with weight $t$, satisfying the condition (iii). Observe finally that it is sufficient to 
ON THE SPECTRAL DECOMPOSITION OF AFFINE HECKE ALGEBRAS 21

consider the case that $x=x_{0}+x^{0} \in Q+Z_{X}$, a sublattice in $X$ of finite index.

By Lie's Theorem we can put the $\pi\left(\theta_{x}\right)$ simultaneously in upper triangular form. Choose $\mathbb{Z}_{+}$-generators $x_{1}, \ldots, x_{m}$ for the cone $Q^{+}$, and a basis $x_{m+1}, \ldots, x_{n}$ for the lattice $Z_{X}$. The Jordan decomposition $\pi\left(\theta_{x_{i}}\right)=D_{i} U_{i}$ gives mutually commuting matrices $D_{i}$ and $U_{i}$, with $D_{i}$ semisimple and $U_{i}$ unipotent upper triangular. By conjugation in the group of invertible upper triangular matrices we may assume that the commuting semisimple matrices $D_{i}$ are diagonal. The strictly upper triangular matrices $M_{i}=\log \left(U_{i}\right)$ are commuting and satisfy

$$
\left.\pi\left(\theta_{x_{i}}\right)\right|_{V_{t}}=\left.x_{i}(t) \exp \left(M_{i}\right)\right|_{V_{t}} .
$$

Hence for $x=\sum_{i=1}^{n} l_{i} x_{i}$, with $l_{i} \in \mathbb{Z}_{+}$when $1 \leq i \leq m$, we have

$$
\left.\pi\left(\theta_{x}\right)\right|_{V_{t}}=x(t) \exp \left(\left.\sum_{i=1}^{n} l_{i} M_{i}\right|_{V_{t}}\right) .
$$

Since $|x(t)| \leq 1$ by assumption, and the exponential map is polynomial of degree $N:=\max _{t}\left\{\operatorname{dim}\left(V_{t}\right)\right\}-1$ on the space of strictly upper triangular matrices commuting with $\pi(\mathcal{A})$, we see that the matrix entries are bounded by a polynomial of degree $N$ in the coefficients $l_{i}$. Observe that $x_{i}\left(2 \rho^{\vee}\right) \in \mathbb{Z}_{>0}$ when $1 \leq i \leq m$. Since the coefficients $l_{i}$ are nonnegative this implies that for all $1 \leq i \leq m, l_{i} \leq x\left(2 \rho^{\vee}\right)$. On the other hand, there exists a constant $d>0$ independent of $x$ such that $\left|l_{i}\right| \leq d\left\|x^{0}\right\|$ for all $i>m$. Thus there exists a constant $d^{\prime}$ independent of $x$ such that $\left|l_{i}\right| \leq d^{\prime} \mathcal{N}(x)$ for all $i$. This gives us the desired estimate of the matrix entries by a polynomial in $\mathcal{N}(x)$, of degree $N$.

Definition 2.21. A representation $\pi$ of $\mathcal{H}$ satisfying the above equivalent conditions is called a tempered representation of $\mathcal{H}$.

Along the same lines one proves:

Lemma 2.22. (Casselman's criterion for discrete series representations.) Let $(V, \pi)$ be a finite dimensional representation of $\mathcal{H}$. The following are equivalent:

(i) $(V, \pi)$ is a subrepresentation of $(\mathfrak{H}, \lambda)$.

(ii) All matrix coefficients of $\pi$ belong to $\mathfrak{H}$.

(iii) The character $\chi$ of $\pi$ belongs to $\mathfrak{H}$.

(iv) The weights $t \in T$ of the generalized $\mathcal{A}$-weight spaces of $V$ satisfy: $|x(t)|<1$, for all $0 \neq x \in X^{+}$.

(v) $Z_{X}=\{0\}$, and there exist $C>0, \epsilon>0$ such that the inequality $\left|m\left(N_{w}\right)\right|<C \mathbf{q}^{-\epsilon l(x)}$ holds for all matrix coefficients $m$ of $\pi$. 
Proof. (i) $\Leftrightarrow$ (ii) Let $E$ denote the projector of $\mathfrak{H}$ onto $V$. Then $E=\rho(e)$ for some idempotent of $\mathfrak{N}$, and since $E$ is open we have $V=\mathcal{H} e \subset$ $\mathfrak{N}$. Choose an orthonormal basis $v_{i}$ of $V$. The corresponding matrix coefficients $\left(v_{i}, x v_{j}\right)=\left(v_{i} v_{j}^{*}, x\right)$ can be identified with the elements $v_{j} v_{i}^{*} \in \mathfrak{H}$. Conversely, suppose that, given a basis $v_{i}$ of $V$ with dual basis $\phi_{j}$ of $V^{*}$, there exist elements $h_{i, j} \in \mathfrak{H}$ such that for all $x \in \mathcal{H}$, $\phi_{i}\left(\pi(x) v_{j}\right)=\left(h_{i, j}^{*}, x\right)$. It follows that for each $i$, the map $v_{j} \rightarrow h_{i, j}$ defines an embedding of $(V, \pi)$ as a subrepresentation of $(\mathfrak{H}, \lambda)$.

(ii) $\Rightarrow$ (iii) $\Rightarrow($ iv) $\Rightarrow$ (v) This is similar to the proof of Lemma 2.20. For the last implication we first remark that (iv) implies that $X^{+}$can not contain $-x$ for any $x \in X^{+}$. Thus $Z_{X}=\{0\}$ in this case, hence $\mathcal{N}(x)=l(x)$.

$(\mathrm{v}) \Rightarrow(\mathrm{ii})$ The number of elements in $W$ with length $l$ grows polynomially in $l$. Thus, by the exponential decay, it is clear that $m=$ $\sum_{w} \overline{m\left(N_{w}^{*}\right)} N_{w}$ is convergent in $\mathfrak{H}$, and moreover $m(x)=\left(m^{*}, x\right)$.

Corollary 2.23. If the $\mathcal{A}$-weights of a finite dimensional representation $(V, \pi)$ of $\mathcal{H}$ satisfy the condition of Lemma 2.22(iv), then $V$ carries a Hilbert structure such that $\pi$ is a *-representation of $\mathcal{H}$, and moreover $\pi$ extends to a representation of $\mathfrak{C}$.

Definition 2.24. Irreducible representations of $\mathcal{H}$ satisfying the equivalent conditions of Lemma 2.22 are called discrete series representations.

\subsection{The Plancherel measure}

Since $\mathfrak{C}$ is separable, liminal and unital, the spectrum $\hat{\mathfrak{C}}$ is a compact $T_{1}$ space with countable base. Moreover it contains an open dense Hausdorff subset.

The algebra $\mathfrak{C}$ comes equipped with the tracial state $\tau$, defining the representations $\lambda, \rho: \mathfrak{C} \rightarrow B(\mathfrak{H})$ of $\mathfrak{C}$ (see Corollary 2.8). The general theory of the decomposition of a trace on a separable, liminal $C^{*}$ algebras (see [15], paragraphe 8.8), asserts that there exists a unique positive Borel measure $\mu_{P l}$ on $\hat{\mathfrak{C}}^{\mathfrak{s}}$ such that

$$
\mathfrak{H} \simeq \int_{\hat{\mathfrak{C}}^{\mathfrak{c}}}^{\oplus} \operatorname{End}\left(V_{\pi}\right) d \mu_{P l}(\pi)
$$

and such that

$$
\tau(h)=\int_{\hat{\mathfrak{C}}} \chi_{\pi}(h) d \mu_{P l}(\pi) .
$$

The measure $\mu_{P l}$ is called the Plancherel measure. 
Theorem 2.25. The support of $\mu_{P l}$ is equal to $\hat{\mathfrak{C}}$. In addition, an irreducible representation $\pi$ of $\mathfrak{C}$ is a subrepresentation of $(\mathfrak{H}, \lambda)$ if and only if $\mu_{P l}(\pi)>0$

Proof. The representation $\lambda_{\tau}=\lambda$ associated with the state $\tau$ is faithful (see Subsection 2.4). The results thus follow from Proposition 8.6.8 of $[15]$.

The center $\mathfrak{Z}$ of $\mathfrak{N}$ will be mapped onto the algebra of diagonalizable operators $L^{\infty}\left(\hat{\mathfrak{C}}, \mu_{P l}\right)$. This is an isomorphism of algebras, continuous when we give $\mathfrak{Z}$ the weak operator topology and $L^{\infty}\left(\hat{\mathfrak{C}}, \mu_{P l}\right)$ the weak topology of the dual of $L^{1}\left(\hat{\mathfrak{C}}, \mu_{P l}\right)$. It is an isometry.

Remark 2.26. In many cases there exist non $\mu_{P l}$-negligible subsets $V$ of $\hat{\mathfrak{C}}$ such that $1<\left|p_{z}^{-1}(y)\right|(<\infty)$ for all $y \in p_{z}(V)$. For instance, the affine Hecke algebra of type $G_{2}$ has two discrete series representations $\pi_{3}, \pi_{2,1}$ associated with the subregular unipotent class $G_{2}(a 1)$ (notation as in [10], Section 13.3) of the complex algebraic group of type $G_{2}$ (also see Appendix 8). Then $p_{z}\left(\pi_{3}\right)=p_{z}\left(\pi_{2,1}\right)$ is equal to the $W_{0}$-orbit of the weighted Dynkin diagram associated with $G_{2}(a 1)$. According to Lemma 2.22 and Theorem 2.25, both $\left\{\pi_{3}\right\}$ and $\left\{\pi_{2,1}\right\}$ are non-negligible.

In such case, the above remarks imply in particular that the weak closure of $\mathcal{Z}$ in $\mathfrak{N}$ is strictly smaller than $\mathfrak{Z}$, the center of $\mathfrak{N}$.

\subsection{Outline of the main results}

This subsection is a continuation of the outline given in 1.0.2.

It is our goal to describe the spectral measure of the tracial state $\tau$ of $\mathfrak{C}$ explicitly. We will not completely succeed, as was explained in 1.0.2(4), but we will obtain a product formula for the density of $\mu_{P l}$ on each component of its support, explicit up to a (intractable) positive real constant factor. An important intermediate step is the description of the more easily accessible spectral measure $\nu$ of the restriction of $\tau$ to the closure $\overline{\mathcal{Z}} \subset \mathfrak{C}$.

2.9.1. Plancherel measure $\nu$ of $\mathcal{Z}$. The subalgebra $\mathcal{Z} \subset \mathcal{H}$ is a *subalgebra. The spectral measure $\nu$ of the restriction of $\tau$ to $\overline{\mathcal{Z}}$ is determined in Subsection 3.4 by the use of the residue calculus.

A residual coset $L \subset T$ is a coset of a subtorus of $T$ such that the pole order of the rational function

$$
\frac{1}{c(t, q) c\left(t^{-1}, q\right)}
$$


along $L$ is equal to $\operatorname{codim}(L)$. Here $c(t, q)$ denotes Macdonald's $c$ function, see equation (3.4). In Appendix 7 this collection of residual cosets is carefully introduced, classified and studied. It turns out to be a finite, $W_{0}$-invariant collection of cosets, with various good properties which play an important role in the calculus of residues (see Subsection 7.3 of Appendix). The residual cosets are of the form (cf. Proposition 7.3 and 7.4) $L=r_{L} T^{L}$, where $T^{L}$ is the connected component of the unit element $e$ in the fix point set in $T$ of the Weyl group $W_{L}$ of a parabolic subsystem $R_{L}$ of $R_{0}$ (a subtorus of $T$ ), and where $r_{L} \in T_{L}=$ $\operatorname{Hom}\left(X_{L}, \mathbb{C}^{\times}\right) \subset T$ is a residual point with respect to the root datum $\mathcal{R}_{L}$ and the restriction $q_{L}$ of $q$ (see Subsection 2.3). This reduces the classification of these cosets to the case of the residual points. The tempered form $L^{\text {temp }}$ of $L=r_{L} T^{L}$ is the compact form of $L$ defined by $L^{\text {temp }}:=r_{L} T_{u}^{L}$.

Using the identification of the space of $W_{0}$-invariant continuous functions on $T$ and the space of continuous functions on $W_{0} \backslash T, \nu$ can be viewed as a $W_{0}$-invariant measure on $T$ supported on $\cup_{L} L^{\text {temp }}$ (union over the residual cosets). We show (cf. Theorem 3.25, Proposition 3.27 and Theorem 3.29) that $\nu=\sum \nu_{L}$, where the sum is over all residual cosets $L$, and where $\nu_{L}$ is a smooth (with respect to the Haar measure $d^{L}$ on $L^{t e m p}$ ) measure with support equal to $L$, such that for all $w \in W_{0}$, $\nu_{w L}=w_{*} \nu_{L}$ (the push forward of $\nu_{L}$ along $\left.w: L^{\text {temp }} \rightarrow(w L)^{\text {temp }}\right)$. There is an explicit (up to a certain rational constant factor $\bar{\kappa}_{W_{L} L}$ ) product formula for $\nu_{L}$, compatible with parabolic induction (Proposition 3.27(iv)):

$$
\nu_{L}\left(r_{L} t^{L}\right)=k_{L} \nu_{\mathcal{R}_{L},\left\{r_{L}\right\}}\left(\left\{r_{L}\right\}\right) m^{L}\left(r_{L} t^{L}\right) d^{L} t^{L},
$$

where $k_{L}=\left|K_{L}\right|$ with $K_{L}=T_{L} \cap T^{L}, m^{L}$ is the rational function (3.57), and $\nu_{\mathcal{R}_{L},\left\{r_{L}\right\}}\left(\left\{r_{L}\right\}\right)$ is the the mass of the residual point $\left\{r_{L}\right\} \subset T_{L}$ with respect to the $W_{L}$-invariant spectral measure $\nu_{\mathcal{R}_{L}}$ on $T_{L}$ determined by $\left(\mathcal{R}_{L}, q_{L}\right)$. In the case where $L=\{r\}$ is a residual point we have (cf. Theorem 3.25)

$$
\nu_{\{r\}}(\{r\})=\bar{\kappa}_{W_{0} r} m_{\{r\}}(r),
$$

where $m_{\{r\}}$ is the given by the product (3.47).

The support of $\nu$ is by definition equal to the image $S$ of the map $p_{z}$ (cf. Proposition 2.13). Thus we conclude from the above description of $\nu$ that $S=W_{0} \backslash \cup_{L} L^{t e m p}$ (union over all residual cosets $L$ with respect to $\mathcal{R}$ and root labels $q$ ). 
ON THE SPECTRAL DECOMPOSITION OF AFFINE HECKE ALGEBRAS 25

2.9.2. Separation by central character. Next we deduce the formula (cf. Corollary 3.23)

$$
\tau(h z)=\int_{T} z(t) \chi_{t}(h) d \nu(t)
$$

for all $z \in \mathcal{Z}$ and $h \in \mathcal{H}$. The $W_{0}$-invariant function $t \rightarrow \chi_{t} \in \mathcal{H}^{*}$ (defined on the support $\cup_{L} L^{\text {temp }}$ of $\nu$, and extended to $T$ by 0 ) has values in the positive tracial states of $\mathfrak{C}$. It follows that $\chi_{t}$ is a finite positive linear combination of irreducible characters of $\mathfrak{C}$, which have central character $W_{0} t$ (cf. Definition 3.24 and Theorem 4.23). In other words, the state $\chi_{t}$ is a positive linear combination of the irreducible characters of $\mathfrak{C}$ in the (finite) fiber $p_{z}^{-1}\left(W_{0} t\right)$. This decomposition of $\chi_{t}$ is the subject of Section 4, and will be described below.

2.9.3. Generic spectrum and residual algebra. The projection $p_{z}: \hat{\mathfrak{C}} \rightarrow$ $S=\hat{\bar{Z}}$ is complicated near non-Hausdorff points of $\hat{\mathfrak{C}}$. Using a variation of techniques introduced in [26] we define an open dense subset $S^{g e n} \subset$ $S$ such that the restriction of $p_{z}$ to $p_{z}^{-1}\left(S^{g e n}\right)$ is a covering map (cf. Theorem 4.39).

The absolute continuity of $\nu_{L}$ with respect to the Haar measure $d^{L}$ on $L^{\text {temp }}$ enables us to disregard a set of positive codimension, so that we can restrict the domain of integration in the above integral to (the pull back to $T$ of) $S^{g e n}$.

Given $t \in T$ such that $W_{0} t \in S^{g e n}$, there exists a unique residual coset $L$ such that $t \in L^{\text {temp }}$. Choose $r_{L} \in T_{L} \cap L$, and write $t=r_{L} t^{L}$ with $t^{L} \in T_{u}^{L}$. The results of [26], suitably adapted, show that in this situation there exists a bijective correspondence between the equivalence classes $\left[\Delta_{\mathcal{R}_{L}, W_{L} r_{L}}\right]$ of irreducible discrete series representations of $\mathcal{H}_{L}:=\mathcal{H}\left(\mathcal{R}_{L}, q_{L}\right)$ with central character $W_{L} r_{L}$ and the equivalence classes of irreducible tempered representations of $\mathcal{H}$ with central character $W_{0} t$. The correspondence is established by an inflation to $\mathcal{H}^{L}:=\mathcal{H}\left(\mathcal{R}^{L}, q^{L}\right)$ using the induction parameter $t^{L} \in T_{u}^{L}$, and induction from $\mathcal{H}^{L}$ to $\mathcal{H}$ (Subsection 4.1, in particular Corollary 4.18).

For $t=r_{L} t^{L} \in L^{\text {temp,gen }}$ such that $R_{L} \subset R_{0}$ is standard parabolic (cf. Theorem 4.23) we obtain

$$
\chi_{t}=\left|W^{L}\right|^{-1} \sum_{\delta \in \Delta_{\mathcal{R}_{L}, W_{L} r_{L}}} d_{\mathcal{R}_{L}, \delta} \chi_{\mathcal{R}_{L}, W_{L} r_{L}, \delta, t^{L}},
$$

where $\chi_{\mathcal{R}_{L}, W_{L} r_{L}, \delta, t^{L}}$ is the character of the representation $\pi_{\mathcal{R}_{L}, W_{L} r_{L}, \delta, t^{L}}$ which is induced from the irreducible discrete series module $\delta$ of $\mathcal{H}_{L}$ (with central character $W_{L} r_{L}$ ) with induction parameter $t^{L}$, and where 
$d_{\mathcal{R}_{L}, \delta}$ denotes the coefficient of the character $\chi_{\delta}$ in the decomposition of the tracial state $\chi_{\mathcal{R}_{L}, r_{L}}$ of $\mathcal{H}_{L}$.

The point is that the coefficients in (2.36) are independent of the induction parameter $t^{L}$. They are certain positive real constants, which we conjecture to be rational, see Conjecture 2.27 below.

The positive real $d_{\mathcal{R}, \delta}$ is the degree of $\delta$ with respect to the finite dimensional "residual Hilbert algebra" $\overline{\mathcal{H}^{r}}$, the quotient of $\mathcal{H}$ with respect to the radical of the positive semi-definite form defined by the tracial state $\chi_{r}$. Hence we have (cf. Corollary 3.32)

$$
\mu_{P l}(\{\delta\})=d_{\mathcal{R}, \delta} \nu\left(\left\{W_{0} r\right\}\right) .
$$

Although Conjecture 2.27 is out of reach for the methods used in this paper, there is a weaker statement which is relatively easy to prove within the context of this paper, and which is already useful for certain applications (see Subsection 6.1). We prove in Section 5 that the real constants $d_{\mathcal{R}, \delta}$ are independent of $\mathbf{q}$, where we assume that $q(s)$ is written in the form Convention 2.1.

2.9.4. Plancherel measure $\mu_{P l}$ and Fourier transform. Equations (2.35) and (2.36) yield a decomposition of the trace $\tau$ in terms of an integral over $t \in S^{r e g}$, where the integration kernel is a sum over the finite fiber $p_{z}\left(W_{0} t\right)$. This integral can be rewritten more sensibly as an integral over a space of "standard induction data" $\Xi$, invariant for the action of a groupoid $\mathcal{W}$ acting on the standard induction data (cf. Subsection 4.5). Next we decompose $\mathcal{W} \backslash \Xi$ in its connected components. This leads to the final formulation of the spectral decomposition of $\mathfrak{H}$ in terms of the Fourier isomorphism $\mathcal{F}$ (cf. Theorem 4.43). This formulation is parallel to the formulation of the Harish-Chandra Plancherel formula for $p$-adic groups, cf. [49], [12].

Let $R_{P} \subset R_{0}$ be standard parabolic root system, and $\delta$ an irreducible discrete series representation of $\mathcal{H}_{P}=\mathcal{H}\left(\mathcal{R}_{P}, q_{P}\right)$ with central character $W_{P} r$. The space $\mathcal{O}$ of all equivalence classes of "twists of $\delta$ ", representations of $\mathcal{H}^{P}$ of the form $\delta_{t^{P}}$ where $t^{P}$ varies over $T_{u}^{P}$, is a compact torus of the form $K_{\delta} \backslash T_{u}^{P}$, where $K_{\delta}$ is the isotropy subgroup of $[\delta]$ in $K_{P}=T^{P} \cap T_{P}$. There exists a principal fiber bundle $\mathcal{V}_{\mathcal{O}}$ over $\mathcal{O}$ whose fiber at $\omega=\left(\mathcal{R}_{P}, W_{P} r_{P}, \delta, K_{\delta} t^{P}\right) \in \mathcal{O}$ is equal to the common representation space $i\left(V_{\delta}\right)$ of the induced representations $\pi\left(\mathcal{R}_{P}, W_{P} r_{P}, \delta, t^{P}\right)$. Thanks to the regularity of certain intertwining operators (see Subsection 4.3) there exists a natural action of the group $W(\mathcal{O})=\left\{w \in W_{0} \mid w\left(R_{P,+}\right)=R_{P,+}\right.$, and $\left.\exists k \in K_{P}: \Psi_{w}(\delta) \simeq \Psi_{k}(\delta)\right\}$ (where $\Psi_{w}(\delta), \Psi_{k}(\delta)$ denote twists of $\delta$ by automorphisms $\psi_{w}, \psi_{k}$ of $\mathcal{H}_{P}$ induced by $w$ and $k$ respectively) on the smooth sections of $\operatorname{End}\left(\mathcal{V}_{\mathcal{O}}\right)$. 
The Fourier transform $\mathcal{F}_{\mathcal{H}}$ is the algebra homomorphism from $\mathcal{H}$ into the direct sum of the algebras of smooth, $W(\mathcal{O})$-equivariant sections of $\operatorname{End}\left(\mathcal{V}_{\mathcal{O}}\right)$, defined by $\left(\mathcal{F}_{\mathcal{H}}(h)\right)(\omega)=\pi\left(\mathcal{R}_{P}, W_{P} r_{P}, \delta, t^{P}\right)(h)$ if $\omega=$ $\left(\mathcal{R}_{P}, W_{P} r_{P}, \delta, K_{\delta} t^{P}\right)$.

In this terminology the Plancherel measure can be expressed as follows (cf. Theorem 4.43):

$$
d \mu_{P l}(\pi(\omega))=\mu_{\mathcal{R}_{P}, P l}(\{\delta\})\left|K_{P} \delta\right| m^{P}(\omega) d^{\mathcal{O}} \omega,
$$

where $K_{P} \delta$ denotes the set of equivalence classes of discrete series representations of $\mathcal{H}_{P}$ in the $K_{P^{-}}$orbit of $\delta, d^{\mathcal{O}}$ is the normalized Haar measure on $\mathcal{O}, m^{P}(\omega)=m^{P}\left(r_{P} t^{P}\right)$ is as in equation (2.33), and $\mu_{\mathcal{R}_{P}, P l}(\{\delta\})$ is given by (2.37) (applied to $\mathcal{R}_{P}$ ). When we equip the space of smooth sections of $\operatorname{End}\left(\mathcal{V}_{\mathcal{O}}\right)^{W(\mathcal{O})}$ with the inner product

$$
\left(f_{1}, f_{2}\right)=\sum_{\mathcal{O} / \sim}|W(\mathcal{O})|^{-1} \int_{\mathcal{O}} \operatorname{tr}\left(f_{1}(\omega)^{*} f_{2}(\omega)\right) d \mu_{P l}(\pi(\omega)),
$$

then the Fourier transform $\mathcal{F}_{\mathcal{H}}$ is an isometry, which extends uniquely to a unitary isomorphism $\mathcal{F}: \mathfrak{H} \rightarrow L^{2}\left(\operatorname{End}\left(\mathcal{V}_{\Xi}\right)\right)^{\mathcal{W}}$.

2.9.5. Further remarks. In [13] we prove that the Fourier isomorphism restricts to an isomorphism of the Schwartz completion $\mathfrak{S}$ of $\mathcal{H}$ (cf. 6.2.2) onto $C^{\infty}\left(\operatorname{End}\left(\mathcal{V}_{\Xi}\right)\right)^{\mathcal{W}}$. Consequently, $\mathfrak{C} \simeq \mathcal{F}(\mathfrak{C})=C\left(\operatorname{End}\left(\mathcal{V}_{\Xi}\right)\right)^{\mathcal{W}}$. In particular, the connected components of $\hat{\mathfrak{C}}^{\mathfrak{E}}$ are the closures $\hat{\mathfrak{C}}_{\mathcal{O}}$ of $\pi\left(\mathcal{O}^{\text {gen }}\right) \subset \hat{\mathfrak{C}}$. We expect that these results will provide an effective approach towards the problem of classification of irreducible tempered modules, using an analog of the analytic R-group (see for example [2]) in our context, granted the classification of the discrete series.

The methods used in this paper are not suitable to obtain a parametrization of the finite set of discrete series representations $\left[\Delta_{W_{0} r}\right]$ with central character $W_{0} r$. If all the labels of $\mathcal{H}$ are equal this information is contained in the work of Kazhdan and Lusztig [23]. They solved this problem using equivariant K-theory in the case when the labels $q_{i}$ are equal, and $X=P$. This result can be extended to the general equal label case, see [38], [41]. In the appendix Section 8 one can find an account of the results of Kazhdan and Lusztig, and the relation with residual cosets.

Let $F$ be a p-adic field and let $\mathcal{G}$ be the group of $F$-rational points in an adjoint semisimple group over $F$ which splits over an unramified extension of $F$. Lusztig [27], [29] solved the above classification problem in principle for any Hecke algebra which arises as the centralizer algebra of a representation of $\mathcal{G}$ which is induced from a cuspidal unipotent representation of (the Levi quotient of) a parahoric subgroup of $\mathcal{G}$. 
The Hecke algebras that are not dealt with by Lusztig are "generic", and these generic algebras are simpler with respect to this problem of parametrization.

Starting from the generic case, Slooten [45] formulated an interesting combinatorics which (among others) conjecturally parametrizes the irreducible tempered modules with real central character for all classical root systems (a generalized Springer correspondence).

For $\delta \in \Delta_{W_{0} r}$ we define $\lambda_{\delta}:=\bar{\kappa}_{W_{0} r}\left|W_{0} r\right| d_{\delta}$, so that

$$
\mu_{P l}(\{\delta\})=\lambda_{\delta} m_{\{r\}}(r)
$$

(see (2.34) and (2.37)). This constant $\lambda_{\delta}$ has been computed explicitly by Mark Reeder in the cases where the Hecke algebra arises as the endomorphism algebra of a representation of a simple p-adic group of exceptional, split adjoint type which is induced from a cuspidal unipotent representation of a parahoric subgroup [40]. He conjectured an interpretation (in this situation) of $\lambda_{\delta}$ (see also [39]) in terms of the Kazhdan-Lusztig parameters of $\delta$. In the exceptional cases he verified this conjecture, using a formula of Schneider and Stuhler [42] for the formal degree of a discrete series representation of an almost simple $p$-adic group which contains fixed vectors for the pro-unipotent radical $U$ of a maximal compact subgroup $K$. This formula of Schneider and Stuhler however is an alternating sum of terms which does not explain the product structure of the formal dimension. In order to rewrite this sum as a product one needs to resort to a case-by-case analysis (computer aided) in [40].

The method of [40] is likely to extend to the general case (joint work with Mark Reeder and Antony Wasserman, in progress). This would imply the following conjecture:

Conjecture 2.27. The $d_{\delta}$ (equivalently, the $\lambda_{\delta}$ ) are rational numbers.

\section{Localization of $\tau$ on $\operatorname{Spec}(\mathcal{Z})$}

Recall the decomposition of $\tau$ we derived in [37], Theorem 3.7:

$$
\tau=\int_{t \in t_{0} T_{u}}\left(\frac{E_{t}}{q\left(w_{0}\right) \Delta(t)}\right) \omega
$$

where $\omega$ denotes the rational $(n, 0)$-form

$$
\omega:=\frac{d t}{c(t, q) c\left(t^{-1}, q\right)}
$$


on $T$. Let us briefly review the various ingredients of this formula. First of all, $T_{u}=\operatorname{Hom}\left(X, S^{1}\right)$, the compact form of the algebraic torus $T=\operatorname{Hom}\left(X, \mathbb{C}^{\times}\right)$, and $t_{0} \in T_{r s}$, the real split part of $T$, and should be deep in the negative chamber $T_{r s,-}$. The precise conditions will be formulated below, see equation (3.7).

The form $d t$ denotes the holomorphic $(n, 0)$-form on $T$ which restricts to the normalized Haar measure on $T_{u}$. It is given by the formula

$$
d t:=(2 \pi i)^{-n}\left(x_{1} x_{2} \ldots x_{n}\right)^{-1} d x_{1} \wedge d x_{2} \wedge \cdots \wedge d x_{n}
$$

if $\left(x_{1}, \ldots, x_{n}\right)$ is a basis of $X$.

The function $\Delta(t):=\prod_{\alpha \in R_{1,+}} \Delta_{\alpha}(t)$ with

$$
\Delta_{\alpha}:=1-\theta_{-\alpha} \in \mathcal{A}
$$

is the Weyl denominator. Here we use the convention to consider the subalgebra $\mathcal{A} \subset \mathcal{H}$ as the algebra of regular functions on $T$ via $\theta_{x}(t):=$ $x(t)$.

The function $c(t)=c(t, q)$ is Macdonald's $c$-function. This $c$-function is introduced as an element of ${ }_{\mathcal{F}} \mathcal{A}$, the field of fractions of $\mathcal{A}$, and will be interpreted as a rational function on $T$ (cf. [37], Definition 1.13). Explicitly, we put

$$
c:=\prod_{\alpha \in R_{0,+}} c_{\alpha}=\prod_{\alpha \in R_{1,+}} c_{\alpha} .
$$

Here we define $c_{\alpha}$ for $\alpha \in R_{1}$ by

$$
c_{\alpha}:=\frac{\left(1+q_{\alpha^{\vee}}^{-1 / 2} \theta_{-\alpha / 2}\right)\left(1-q_{\alpha^{\vee}}^{-1 / 2} q_{2 \alpha^{\vee}}^{-1} \theta_{-\alpha / 2}\right)}{1-\theta_{-\alpha}} \in{ }_{\mathcal{F}} \mathcal{A} .
$$

If $\alpha \in R_{0} \backslash R_{1}$ then we define $c_{\alpha}:=c_{2 \alpha}$.

Remark 3.1. We have thus associated a c-function $c_{\alpha}$ to each root $\alpha \in R_{n r}$, but $c_{\alpha}$ only depends on the direction of $\alpha$. This convention is different from the one used in [37]. It is handy to write the formulas for the $c$ functions in the above form, but strictly speaking incorrect if $\alpha \in R_{1}$ and $\alpha / 2 \notin X$. However, we formally put $q_{2 \alpha^{\vee}}=1$ if $\alpha / 2 \notin R_{0}$, and then rewrite the numerator as $\left(1-q_{\alpha^{\vee}}^{-1} \theta_{-\alpha}\right)$. Here and below we use this convention.

The exact inequalities which have to be met by $t_{0} \in T_{r s}$ in order to represent the trace functional $\tau$ are as follows. If $q(s)>1$ for all $s \in S^{\text {aff }}$, then according to [37], Definition 1.4 and Corollary 3.2, the representation (3.1) holds if

$$
\forall \alpha \in F_{0}: \alpha\left(t_{0}\right)<q_{\alpha^{\vee}}^{-1} q_{\alpha^{\vee} / 2}^{-1 / 2} .
$$


It is clear that this representation of $\tau$ remains true if we vary the parameters $q$ in a connected open set $U$ such that $\{q \mid \forall s: q(s)>$ $1\} \subset U \subset\{q \mid \forall s: q(s)>0\}$, as long as the poles of the kernel of the integral for any $q \in U$ do not intersect the integration cycle $t_{0} T_{u}$. It follows that the representation (3.1) of $\tau$ holds for any $q$ such that $\forall s \in S^{\text {aff }}: q(s)>0$, provided that

$$
\forall \alpha \in F_{0}: \alpha\left(t_{0}\right)<\min \left\{\left(q_{\alpha^{\vee}} q_{\alpha^{\vee} / 2}^{1 / 2}\right)^{ \pm 1}, q_{\alpha^{\vee} / 2}^{ \pm 1 / 2}\right\}
$$

Observe that

$$
q_{\alpha^{\vee}} q_{\alpha^{\vee} / 2}^{1 / 2}=q_{\alpha^{\vee}}^{1 / 2} q_{\alpha^{\vee}+1}^{1 / 2} ; q_{\alpha^{\vee} / 2}^{1 / 2}=q_{\alpha^{\vee}}^{-1 / 2} q_{\alpha^{\vee}+1}^{1 / 2}
$$

The expression $E_{t} \in \mathcal{H}^{*}$ is the holomorphic Eisenstein series for $\mathcal{H}$, with the following defining properties (cf. [37], Propositions 2.23 and 2.24):

$$
\begin{aligned}
& \text { (i) } \forall h \in \mathcal{H} \text {, the map } T \ni t \rightarrow E_{t}(h) \text { is regular. } \\
& \text { (ii) } \forall x, y \in X, h \in \mathcal{H}, E_{t}\left(\theta_{x} h \theta_{y}\right)=t(x+y) E_{t}(h) . \\
& \text { (iii) } E_{t}(1)=q\left(w_{0}\right) \Delta(t) .
\end{aligned}
$$

We want to rewrite the integral (3.1) representing the trace functional as an integral over the collection of tempered residual cosets, by a contour shift. It turns out that such a representation exists and is unique. To find it, we need an intermediate step. We will first rewrite the integral as a sum of integrals over a larger set of tempered "quasiresidual cosets", and then we will show that if we symmetrize the result over $W_{0}$, all the contributions of non-residual cosets cancel.

\section{1. $\omega$-residual cosets}

The basic scheme to compute residues has nothing to do with the properties of root systems. It is therefore convenient to formulate everything in a more general setting first. Later we will consider the consequences that are specific to our context.

Let $T$ be a complex algebraic torus with character lattice $X$.

Definition 3.2. Let $\omega=p d t / q$ be a rational $(n, 0)$-form on $T$. Assume that $p, q$ are of the form

$$
q(t)=\prod_{m \in \mathcal{M}}\left(d_{m}^{-1} x_{m}(t)-1\right) ; p(t)=\prod_{m^{\prime} \in \mathcal{M}^{\prime}}\left(d_{m^{\prime}}^{-1} x_{m^{\prime}}(t)-1\right),
$$

where the products are taken over finite multisets $\mathcal{M}, \mathcal{M}^{\prime}$. The multisets $\mathcal{M}$ and $\mathcal{M}^{\prime}$ come equipped with maps $m \rightarrow\left(x_{m}, d_{m}\right) \in X \times \mathbb{C}^{\times}$. 
For $m \in \mathcal{M} \cup \mathcal{M}^{\prime}$ we denote by $L_{m} \subset T$ the codimension 1 subvariety $L_{m}=\left\{t \mid x_{m}(t)=d_{m}\right\}$, and we denote by $D_{\omega}$ the divisor $\sum_{m \in \mathcal{M}} L_{m}-\sum_{m^{\prime} \in \mathcal{M}^{\prime}} L_{m^{\prime}}$ on $T$ of $q / p$.

An $\omega$-residual coset $L$ is a connected component of $\cap_{m \in J} L_{m}$ for some $J \subset \mathcal{M}$, such that the pole order $i_{L}$ of $\omega$ along $L$ satisfies

$$
i_{L}:=\left|\left\{m \in \mathcal{M} \mid L \subset L_{m}\right\}\right|-\left|\left\{m^{\prime} \in \mathcal{M}^{\prime} \mid L \subset L_{m^{\prime}}\right\}\right| \geq \operatorname{codim}(L) .
$$

The collection of $\omega$-residual cosets is denoted by $\mathcal{L}^{\omega}$. This is a finite, nonempty collection of cosets of subtori of $T$, which includes by definition $T$ itself (the empty intersection of the cosets $L_{m}$ ).

Note that $\omega$ as in the above definition is completely determined by the divisor $D_{\omega}$ on $T$.

Let $\langle\cdot, \cdot\rangle$ be a rational inner product on the vector space $\mathbb{Q} \otimes Y$, where $Y$ is the cocharacter lattice of $T$. This defines an isomorphism between $\mathbb{Q} \otimes X$ and $\mathbb{Q} \otimes Y$, and we also denote by $\langle\cdot, \cdot\rangle$ the corresponding inner product on $\mathbb{Q} \otimes X$. Through the exponential map exp : $\mathfrak{t}_{\mathbb{C}}:=\mathbb{C} \otimes Y \rightarrow T$ we obtain a distance function on $T$. It is defined by taking the distance between $2 \pi i Y$-orbits in $\mathfrak{t}_{\mathbb{C}}$. We denote by $|t|$ the distance of $t \in T$ to $e \in T$.

Suppose that $L$ is a connected component of the intersection $\cap_{m \in J} L_{m}$ for some subset $J \subset \mathcal{M}$. Then $L$ is a coset for the connected component of $e$ of the subgroup $\cap_{m \in J} T^{m} \subset T$, where $T^{m}:=\left\{t \in T \mid x_{m}(t)=1\right\}$. We denote this connected component by $T^{L} \subset T$. Its character lattice $X^{L}:=\operatorname{Hom}\left(T^{L}, \mathbb{C}^{\times}\right)$is equal to the quotient $X^{L}=X /\left(\left(\sum_{m \in J} \mathbb{Q} x_{m}\right) \cap\right.$ $X)$. Let $X_{L}$ be the quotient $X_{L}:=X /\left(\cap_{m \in J} x_{m}^{\perp} \cap X\right)$. Then $T_{L}:=$ $\operatorname{Hom}\left(X_{L}, \mathbb{C}^{\times}\right)$is an algebraic subtorus of $T$, the subtorus "orthogonal to $T^{L}$ ". The intersection $K_{L}:=T_{L} \cap T^{L}$ is a finite abelian group, and is canonically isomorphic to character group of the quotient $X /\left(X_{L}+X^{L}\right)$. It follows that $L \cap T_{L}$ is a coset for the finite subgroup $K_{L} \subset T_{u}$.

We denote by $\mathcal{M}_{L} \subset \mathcal{M}$ the subset $\left\{m \in \mathcal{M} \mid x_{m}(L)=d_{m}\right\}$. We choose an element $r_{L}=s_{L} c_{L} \in T_{L} \cap L$ for each $L$ so that we can write $L=r_{L} T^{L}$. We call $c_{L} \in T_{r s}$ the center of $L$, and note that this center is determined uniquely by $L$. We write $c_{L}=\exp \gamma_{L}$ with $\gamma_{L} \in \mathfrak{t}_{L}$. The set of centers of the $\omega$-residual cosets is denoted by $\mathcal{C}^{\omega}$. The tempered form of a $\omega$-residual $L=r_{L} T^{L}$ is by definition $L^{\text {temp }}:=r_{L} T_{u}^{L}$ (which is independent of the choice of $r_{L}$ ), and such a coset will be called an $\omega$-tempered coset.

Basically, the only properties of the collection $\mathcal{L}^{\omega}$ we will need are

Proposition 3.3. $\quad$ (i) If $c \in \mathcal{C}^{\omega}$ then the union

$$
S_{c}:=\cup_{\left\{L \in \mathcal{L}^{\omega} \mid c_{L}=c\right\}} L^{\text {temp }} \subset c T_{u}
$$


is a regular support in the sense of [43] in $c T_{u}$. This means that a distribution on $c T_{u}$ with support in $S_{c}$ can be written as a sum of derivatives of push forwards of measures on $S_{c} \subset c T_{u}$.

(ii) If $c=\exp \gamma \in T_{r s}$, and $L$ is $\omega$-residual with $\left|\gamma_{L}\right| \geq|\gamma|$ but $\gamma_{L} \neq$ $\gamma$, then there exists a $m \in \mathcal{M}_{L}$ such that $f(t)=x_{m}(t)-d_{m}$ is non-vanishing on $c T_{u}$.

Proof. The set $S_{c}$ is a finite union of smooth varieties, obviously satisfying the condition of [43], Chapitre III, $\S 9$ for regularity, proving (i). As for (ii), first note that the assumption implies that $\gamma_{L} \neq 0$, hence that $L \neq T$. Thus the codimension of $L$ is positive, and $\mathcal{M}_{L} \neq \emptyset$. Clearly $\gamma \notin \gamma_{L}+\mathfrak{t}^{L}=\log \left(T_{r s} \cap L T_{u}\right)$ since $\gamma_{L}$ is the unique smallest vector in this affine linear space. Because $\left\{x_{m} \mid m \in \mathcal{M}_{L}\right\}$ spans $\mathfrak{t}_{L}=\left(\mathfrak{t}^{L}\right)^{\perp}$, we can find a $m \in \mathcal{M}_{L}$ such that $x_{m}(\gamma) \neq x_{m}\left(\gamma_{L}\right)$. This implies the result.

\subsection{The contour shift and the local contributions}

The following lemma is essentially the same as Lemma 3.1 of [18], but because of its basic importance we have included the proof here, adapted to the present context. See also [5] for a more general method in the same spirit.

Lemma 3.4. Let $\omega$ be as in Definition 3.2 and let $t_{0} \in T_{r s} \backslash \cup\left(T_{r s} \cap\right.$ $\left.T_{u} L_{m}\right)$. Fix an inner product $\langle\cdot, \cdot\rangle$ on $\mathbb{Q} \otimes Y$. Then there exists a unique collection of distributions $\left\{\mathfrak{X}_{c} \in C^{-\infty}\left(c T_{u}\right)\right\}_{c \in C^{\omega}}$ such that the following conditions hold:

(i) The support of $\mathfrak{X}_{c}$ satisfies $\operatorname{supp}\left(\mathfrak{X}_{c}\right) \subset S_{c}$ (cf. Proposition 3.3).

(ii) For every $a \in \mathcal{A}^{\text {an }}(T)$ (the ring of analytic functions on $T$ ) we have

$$
\int_{t \in t_{0} T_{u}} a(t) \omega(t)=\sum_{c \in \mathcal{C}^{\omega}} \mathfrak{X}_{c}\left(\left.a\right|_{c T_{u}}\right) .
$$

Proof. The existence is proved by induction on the dimension $n$ of $T$, the case of $n=0$ being trivial. Suppose that the result is true for tori of dimension $n-1$. Choose a smooth path in $T_{r s}$ from $t_{0}$ to the identity $e$ which intersects the real projection $L_{m, r}=T_{r s} \cap T_{u} L_{m}$ of the codimension 1 cosets $L_{m}$ transversally and in at most one point $t\left(L_{m, r}\right)$. We may assume that the intersection points are mutually distinct with possible exception for the cases $t\left(L_{m, r}\right)=e$, i.e. when $e \in L_{m, r}$. When we move $t_{0}$ along the curve towards $e$, then we pick up residues when we pass at a point $t=t\left(L_{m, r}\right) \neq e$ on the curve. We may assume that 
the cosets $L_{m}$ are connected (by factoring the defining equations, and adapting $\mathcal{M}$ accordingly). Let $L=L_{m}$ be such that $t \in L_{m, r}$. For simplicity of notation we write $(x, d)$ instead of $\left(x_{m}, d_{m}\right)$. Recall the decomposition $L=r_{L} T^{L}=s_{L} c_{L} T^{L}$ with $r_{L} \in T_{L}$. Let $d^{L} t$ denote the holomorphic extension to $T^{L}$ of the normalized Haar measure on $T_{u}^{L}$, and similarly for $d_{L} t$ on $T_{L}$. Let $K_{L}=T^{L} \cap T_{L}$, and let $k_{L}$ be its order. The product homomorphism $\pi: T^{L} \times T_{L} \rightarrow T$ has kernel $\left\{\left(k, k^{-1}\right) \mid k \in K_{L}\right\}$. The residue that is picked up on $L$ when we cross at $t$ can be written as follows:

$$
\int_{t^{L} \in T_{u}^{L}} \sum_{k \in K_{L}} \int_{t_{L} \in k C}(a p / q)\left(t s_{L} t^{L} t_{L}\right) d_{L}\left(t_{L}\right) d^{L}\left(t^{L}\right),
$$

where $C$ denotes a small circle in $T_{L} \simeq \mathbb{C}^{\times}$around 1 . Using the action of $\operatorname{Ker}(\pi)$ and the invariance of $d^{L}$ and $d_{L}$, and in addition using $r_{L}$ as a base point of $L$, this equals

$$
k_{L} \int_{t^{L} \in t c_{L}^{-1} T_{u}^{L}} \int_{t_{L} \in C}(a p / q)\left(r_{L} t^{L} t_{L}\right) d_{L}\left(t_{L}\right) d^{L}\left(t^{L}\right) .
$$

Let $x_{L} \in X_{L}$ be a generator of $X_{L}$. Let $D$ be the holomorphic constant vector field on $T_{L}$ which is dual to $x_{L}$. We extend $D$ to a constant holomorphic vector field on $T$. We define a $k_{L}$-th root of $d$ by $x_{L}\left(r_{L}\right)=d^{1 / k_{L}}$, so that the pull back of $d^{-1} x-1$ to $T_{L} \times T^{L}$ factors as follows:

$$
\left(d^{-1} x-1\right)=\prod_{k \in K_{L}}\left(x_{L}\left(k^{-1}\right) d^{-1 / k_{L}} x_{L}-1\right):=\prod_{k \in K_{L}}\left(d_{k}^{-1} x_{L}-1\right) .
$$

With these notations, the above residue contribution is of the form

$$
\left.\int_{t^{\prime} \in t c_{L}^{-1} T_{u}^{L}}\left(B_{i_{L}-1}(D)\left(\left(\left(d_{k}^{-1} x_{L}-1\right)^{i_{L}} p / q\right) a\right)\left(r_{L} t^{L} t_{L}\right)\right)\right|_{t_{L}=1} d^{L}\left(t^{L}\right),
$$

where $B_{j}(T) \in \mathbb{Q}[T]$ is a certain polynomial of degree $j$. Note that there may exist other $L_{m^{\prime}}$ with $t \in L_{m^{\prime}, r}$. We pick up similar residues with respect to these cosets as well when we cross at $t$.

The above integral can be rearranged as follows

$$
\sum_{j=0}^{i_{L}-1} \int_{t s_{L} T_{u}^{L}}\left(\left.D^{j}(a)\right|_{L}\right) \omega_{j}
$$

where $\omega_{j}$ is itself a rational $(n-1,0)$-form on $L$ which is a linear combination

$$
\omega_{j}=\sum_{i} f_{j, i} \omega_{j, i}
$$


on $L$ with regular holomorphic coefficients $f_{j, i}$, and $(n-1,0)$-forms $\omega_{j, i}$ which factor as in Definition 3.2. The forms $\omega_{j, i}$ have poles along the intersections $L_{n}^{\prime}=L \cap L_{n}$ (with $n \in \mathcal{M}$ ) which are of codimension 1 in $L$. A simple computation shows that we can choose this decomposition of $\omega_{j}$ such that for every $j, i$ and every connected component $H$ of an intersection of cosets of the form $L_{n}^{\prime} \subset L$, the index $i_{\omega_{j, i}, H}$ of $H \subset L$ satisfies $i_{\omega_{j, i}, H} \leq\left(i_{\omega, H}-1\right)-j$. It follows that the union over all $j, i$ of the $\omega_{j, i}$-residual cosets in $L$ is contained in the collection of $\omega$-residual cosets of $T$. Moreover, when we take $r_{L}$ as a base point of $L$, so that we identify $L$ with $T^{L}$ through the map $t^{L} \rightarrow r_{L} t^{L}$, then the tempered form of a $\omega_{j, i}$-residual coset in $L$ is equal to its tempered form as a $\omega$ residual coset in $T$. By the induction hypotheses we can thus rewrite the residue on $L$ in the desired form, where the role of the identity element in the coset $L$ is now played by $r_{L}$.

At the identity $e \in T$ itself we have to take a boundary value of $\omega$ towards $T_{u}$, which defines a distribution on $T_{u}$. This proves the existence.

The uniqueness is proved as follows. Suppose that we have a collection $\left\{\mathfrak{Y}_{c} \in C^{-\infty}\left(c T_{u}\right)\right\}_{c \in \mathcal{C}}$ of distributions such that

(i) $\operatorname{supp}\left(\mathfrak{Y}_{c}\right) \subset S_{c}$.

(ii) $\forall a \in \mathbb{C}[T]: \sum_{c \in \mathcal{C} \omega} \mathfrak{Y}_{c}\left(\left.a\right|_{c T_{u}}\right)=0$.

We show that $\mathfrak{Y}_{c}=0$ by induction on $|\gamma=\log (c)|$. Choose $c \in \mathcal{C}^{\omega}$ such that $\mathfrak{Y}_{c^{\prime}}=0$ for all $c^{\prime}$ with $\left|\gamma^{\prime}\right|<|\gamma|$. For each $L \in \mathcal{L}^{\omega}$ with $\left|\gamma_{L}\right| \geq|\gamma|$ and $\gamma_{L} \neq \gamma$ we choose a $l \in \mathcal{M}_{L}$ such that $x_{l}(t)-d_{l}$ does not vanish on $c T_{u}$ (Proposition 3.3) and we set

$$
\nu(t):=\prod_{\left\{L:\left|\gamma_{L}\right| \geq|\gamma| \text { and } \gamma_{L} \neq \gamma\right\}}\left(x_{l}(t)-d_{l}\right) .
$$

It is clear that for sufficiently large $N \in \mathbb{N}, \mathfrak{Y}_{c}\left(\nu^{N} a\right)=0$ for all $a \in$ $\mathbb{C}[T]$. On the other hand, by the theory of Fourier series of distributions on $T_{u},\left.\mathbb{C}[T]\right|_{c T_{u}}$ is a dense set of test functions on $c T_{u}$. Since $\nu^{N}$ is nonvanishing on $c T_{u}$, this function is a unit in the space of test functions in $c T_{u}$. Thus also $\left.\nu^{N} \mathbb{C}[T]\right|_{c T_{u}}$ is dense in the space of test functions. It follows that $\mathfrak{Y}_{c}=0$.

3.2.1. Approximating sequences. There is an "analytically dual" formulation of the result on residue distributions that will be useful later on. The idea to deal with the residue distributions in this way was inspired by the approach in [21] to prove the positivity of certain residual spherical functions. 
ON THE SPECTRAL DECOMPOSITION OF AFFINE HECKE ALGEBRAS 35

Lemma 3.5. For all $N \in \mathbb{N}$ there exists a collection of sequences $\left\{a_{n}^{N, c}\right\}_{n \in \mathbb{N}}\left(c \in \mathcal{C}^{\omega}\right)$ in $\mathcal{A}$ with the following properties:

(i) For all $n \in \mathbb{N}, \sum_{c \in \mathcal{C} \omega} a_{n}^{N, c}=1$.

(ii) For every holomorphic constant coefficient differential operator $D$ of order at most $N$ on $T, D\left(a_{n}^{N, c}\right) \rightarrow D(1)$ uniformly on $S_{c}$ and $D\left(a_{n}^{N, c}\right) \rightarrow 0$ on $S_{c^{\prime}}$ if $c^{\prime} \neq c$.

Proof. We construct the sequences with induction on the norm $\mid \gamma=$ $\log (c) \mid$. We fix $N$ and suppress it from the notation. Let $c \in \mathcal{C}^{\omega}$ and assume that we have already constructed such sequences $a_{n}^{c^{\prime}}$ satisfying (ii) for all $c^{\prime}$ with $\left|\gamma^{\prime}\right|<|\gamma|$. Consider the function $\nu$ constructed in the second part of the proof of Lemma 3.4. By Fourier analysis on $c T_{u}$ it is clear that there exists a sequence $\left\{\phi_{n}\right\}_{n \in \mathbb{N}}$ in $\mathbb{C}[T]$ such that for each holomorphic constant coefficient differential operator $D$ of order at most $N$ there exists a constant $c_{D}$ such that

$$
\left\|\left.\left(D\left(\phi_{n}\right)-D\left(\nu^{-(N+1)}\right)\right)\right|_{c T_{u}}\right\|_{\infty}<c_{D} / n
$$

Applying Leibniz' rule to $\nu^{(N+1)} \phi_{n}-1=\nu^{(N+1)}\left(\phi_{n}-\nu^{-(N+1)}\right)$ repeatedly we see that this implies that there exists a constant $c_{D}^{\prime}$ for each holomorphic constant coefficient differential operator $D$, such that

$$
\left\|\left.\left(D\left(\nu^{(N+1)} \phi_{n}\right)-D(1)\right)\right|_{c T_{u}}\right\|_{\infty}<c_{D}^{\prime} / n \text {. }
$$

Notice that $D\left(\nu^{(N+1)} \phi_{n}\right)=0$ on all $S_{c^{\prime}}$ with $\left|\gamma^{\prime}\right| \geq|\gamma|$ but $\gamma^{\prime} \neq \gamma$. On the other hand, for each holomorphic constant coefficient differential operator $E$ the function $E\left(1-\sum_{\left\{c^{\prime}|| \gamma^{\prime}|<| \gamma \mid\right\}} a_{k}^{c^{\prime}}\right)$ converges uniformly to zero on each $S_{c^{\prime}}$ with $\left|\gamma^{\prime}\right|<|\gamma|$. Again applying Leibniz' rule repeatedly we see that there exist a $k \in \mathbb{N}$ (depending on $n$ ) such that the function

$$
a_{n}^{c}:=\nu^{(N+1)} \phi_{n}\left(1-\sum_{\left\{c^{\prime}|| \gamma^{\prime}|<| \gamma \mid\right\}} a_{k}^{c^{\prime}}\right)
$$

has the property that

$$
\left\|\left.D\left(a_{n}^{c}\right)\right|_{\cup S_{c^{\prime}}}\right\|_{\infty}<c_{D}^{\prime} / n
$$

where the union is taken over all $c^{\prime}$ with $\left|\gamma^{\prime}\right|<|\gamma|$. It is clear that the sequence $a_{n}^{c}$ thus constructed satisfies (ii). We continue this process until we have only one center $c$ left. For this last center we can simply put

$$
a_{n}^{c}:=1-\sum_{c^{\prime} \neq c} a_{n}^{c^{\prime}} .
$$

This satisfies the property (ii), and forces (i) to be valid.

The use of such collections of sequences is the following: 
Proposition 3.6. In the situation of Lemma 3.4 and given any collection of sequences $\left\{a_{n}^{c}\right\}$ as constructed in Lemma 3.5 we can express the residue distributions as (with $a \in \mathcal{A}$ ):

$$
\mathfrak{X}_{c}(a)=\lim _{n \rightarrow \infty} \tau\left(a_{n}^{c} a\right),
$$

provided $N$ (in Lemma 3.5) is chosen sufficiently large.

Proof. Because we are working with distributions on compact spaces, the orders of the distributions are finite. Take $N$ larger than the maximum of all orders of the $\mathfrak{X}_{c}$. By Proposition 3.3 we can thus express $\mathfrak{X}_{c^{\prime}}$ as a sum of derivatives of order at most $N$ of measures supported on $S_{c^{\prime}}$. The result now follows directly from the defining properties of the sequence $a_{n}^{c}$.

3.2.2. Cycles of integration. Yet another useful way to express the residue distribution is by means of integration of $a \omega$ over a suitable compact n-cycle. The results of this subsection will be needed later on to compute certain residue distributions at "generic" points of their support.

In the proposition below we will use the distance function on $T$ which measures the distance between $2 \pi i Y$-orbits in $\mathfrak{t}_{\mathbb{C}}$. For $\delta>0$ and each $L$ which is a connected component of an intersection of codimension 1 cosets $L_{m} \subset T$ with $m \in \mathcal{M}$, we denote by $\mathcal{B}_{L}\left(r_{L}, \delta\right)$ a ball in $T_{L}$ with radius $\delta$ and center $r_{L}$, and by $\mathcal{B}_{r s}^{L}(\delta)$ a ball with radius $\delta$ and center $e$ in $T_{r s}^{L}$. We put $\mathcal{M}_{L} \subset \mathcal{M}$ for the $m \in \mathcal{M}$ such that $L \subset L_{m}$, and $\mathcal{M}^{L} \subset \mathcal{M}$ for the $m \in \mathcal{M}$ such that $L_{m} \cap L$ has codimension 1 in $L$. We write $T^{m}=\left\{t \mid x_{m}(t)=1\right\}$.

Let $U^{L}(\delta) \subset T^{L}$ be the open set $\left\{t \in T^{L} \mid \forall m \in \mathcal{M}^{L}: t \overline{\mathcal{B}_{L}\left(r_{L}, \delta\right)} \cap\right.$ $\left.L_{m}=\emptyset\right\}$. Note that $U^{L}\left(\delta_{1}\right) \subset U^{L}\left(\delta_{2}\right)$ if $\delta_{1}>\delta_{2}$, and that the union of these open sets is equal to the complement of union of the codimension 1 subsets $r_{L}^{-1}\left(L \cap L_{m}\right) \subset T^{L}$ with $m \in \mathcal{M}^{L}$.

Proposition 3.7. Let $\epsilon>0$ be such that for all $m \in \mathcal{M}$ and $L \in \mathcal{L}^{\omega}$, $L_{m} \cap \mathcal{B}_{L}\left(r_{L}, \epsilon\right) \mathcal{B}_{r s}^{L}(\epsilon) T_{u}^{L} \neq \emptyset$ implies that $L^{\text {temp }} \cap L_{m} \neq \emptyset$. Denote by $\mathcal{M}^{L, \text { temp }}$ the set of $m \in \mathcal{M}^{L}$ such that $L^{\text {temp }} \cap L_{m} \neq \emptyset$. There exist

(i) $\forall L \in \mathcal{L}^{\omega}$, a point $\epsilon^{L} \in \mathcal{B}_{r s}^{L}(\epsilon) \backslash \cup_{m \in \mathcal{M}^{L, t e m p}} T^{m}$,

(ii) $a 0<\delta<\epsilon$ such that $\forall L \in \mathcal{L}^{\omega}, \epsilon^{L} T_{u}^{L} \subset U^{L}(\delta)$, and

(iii) $\forall L \in \mathcal{L}^{\omega}$, a compact cycle $\xi_{L} \subset \mathcal{B}_{L}\left(r_{L}, \delta\right) \backslash \cup_{m \in \mathcal{M}_{L}} L_{m}$ of $d i$ mension $\operatorname{dim}_{\mathbb{C}}\left(T_{L}\right)$,

such that

$$
\forall c \in \mathcal{C}^{\omega}, \forall \phi \in C^{\infty}\left(c T_{u}\right): \mathfrak{X}_{c}(\phi)=\sum_{\left\{L \mid c_{L}=c\right\}} \mathfrak{X}_{L}(\phi)
$$


ON THE SPECTRAL DECOMPOSITION OF AFFINE HECKE ALGEBRAS 37

where $\mathfrak{X}_{L}$ is the distribution on $c T_{u}$ with support $L^{\text {temp }}$ defined by

$$
\forall a \in \mathcal{A}: \mathfrak{X}_{L}(a)=\int_{\epsilon^{L} T_{u}^{L} \times \xi_{L}} a \omega
$$

If $\mathcal{M}^{L, t e m p}=\emptyset$ we may take $\epsilon^{L}=e$.

Proof. We begin the proof by remarking that (i), (ii) and (iii) imply that the functional $\mathfrak{X}_{L}$ on $\mathcal{A}$ indeed defines a distribution on $c_{L} T_{u}$, supported on $L^{t e m p}$. Consider for $t \in U^{L}(\delta)$ the inner integral

$$
\int_{t \xi} a \omega:=i(a, t) d^{L} t .
$$

Then $i(a, t)$ is a linear combination of (possibly higher order) partial derivatives $D_{\kappa} a$ of $a$ at $r_{L} t$ in the direction of $T_{L}$, with coefficients in the ring of meromorphic functions on $T^{L}$ which are regular outside the codimension 1 intersections $r_{L}^{-1}\left(L \cap L_{m}\right)$ :

$$
i(a, t)=\sum_{\kappa} f_{\kappa} D_{\kappa} a .
$$

Hence $\mathfrak{X}_{L}(a)$ is equal to the sum of the boundary value distributions $\mathrm{BV}_{\epsilon^{L}, f_{\kappa}}$ of the meromorphic coefficient functions, applied to the corresponding partial derivative $D_{\kappa} a$ of $a$, restricted to $L^{t e m p}$ :

$$
\mathfrak{X}_{L}(a)=\sum_{\kappa} \mathrm{BV}_{\epsilon^{L}, f_{\kappa}}\left(\left.D_{\kappa} a\right|_{L^{t e m p}}\right) .
$$

We see that $\mathfrak{X}_{L}$ is a distribution supported in $L^{\text {temp }} \subset c_{L} T_{u}$, which only depends on $\xi_{L}$ and on the component of $\mathcal{B}_{r s}^{L}(\epsilon) \backslash \cup_{m \in \mathcal{M}^{L, t e m p}} T^{m}$ in which $\epsilon^{L}$ lies.

Hence, by the uniqueness assertion of Lemma 3.4, we conclude that it is sufficient to prove that we can choose $\epsilon^{L}, \delta, \xi_{L}$ in such a way that

$$
\forall a \in \mathcal{A}: \int_{t_{0} T_{u}} a \omega=\sum_{L \in \mathcal{L}^{\omega}} \mathfrak{X}_{L}(a) .
$$

In order to prove this it is enough to show that we can choose $\epsilon^{L}, \delta, \xi_{L}$ as in (i), (ii) and (iii) for the larger collection $\tilde{\mathcal{L}}^{\omega}$ of all the connected components of intersections of the $L_{m}$ (with $m \in \mathcal{M}$ ), such that

$$
t_{0} T_{u} \sim \cup_{L \in \tilde{\mathcal{L}}^{\omega}} \epsilon^{L} T_{u}^{L} \times \xi_{L} .
$$

Here $\sim$ means that the left hand side and the right hand side are homologous cycles in $T \backslash \cup_{m \in \mathcal{M}} L_{m}$. The desired result follows from this, since the functional $\mathfrak{X}_{L}$ is equal to 0 unless $L$ is $\omega$-residual (because the 
inner integral (3.14) is identically equal to 0 for non-residual intersections, by an elementary argument which is given in detail in the proof of Theorem 3.25).

Let $k \in\{0,1, \ldots, n-1\}$. Denote by $\tilde{\mathcal{L}}^{\omega}(k)$ the collection of connected components of intersections of the $L_{m}(m \in \mathcal{M})$ such that $\operatorname{codim}(L)<$ $k$. Assume that we already have constructed points $\epsilon^{L}, \delta, \xi_{L}$ satisfying (i), (ii) and (iii) for all $L \in \tilde{\mathcal{L}}^{\omega}(k)$ and in addition, for each $L \in \tilde{\mathcal{L}}^{\omega}$ with $\operatorname{codim}(L)=k$, a finite set of points $\Omega_{L} \subset T_{r s}^{L}$ such that $\Omega_{L} T_{u}^{L} \subset U^{L}(\delta)$ and a cycle $\xi_{L, w} \subset \mathcal{B}_{L}\left(r_{L}, \delta\right) \backslash \cup_{m \in \mathcal{M}_{L}} L_{m}$ for each $w \in \Omega_{L}$, such that $t_{0} T_{u}$ is homologous to

$$
\cup_{L \in \tilde{\mathcal{L}}^{\omega}(k)}\left(\epsilon^{L} T_{u}^{L} \times \xi_{L}\right) \cup \cup_{L \in \tilde{\mathcal{L}}^{\omega}(k+1) \backslash \tilde{\mathcal{L}}^{\omega}(k)} \cup_{w \in \Omega_{L}}\left(w T_{u}^{L} \times \xi_{L, w}\right) .
$$

This equation holds for $k=0$, with $\Omega_{T}=\left\{t_{0}\right\}$, which is the starting point of the inductive construction to be discussed below. We will construct $\epsilon^{L}, \delta_{1}$ and $\xi_{L}$ for $L \in \tilde{\mathcal{L}}^{\omega}(k+1) \backslash \tilde{\mathcal{L}}^{\omega}(k)$, and finite sets $\Omega_{L}$ for $L \in \tilde{\mathcal{L}}^{\omega}(k+2) \backslash \tilde{\mathcal{L}}^{\omega}(k+1)$, with a cycle $\xi_{w}$ for each $w \in \Omega_{L}$ such that equation (3.19) holds with $k$ replaced by $k+1$, and $\delta$ by $\delta_{1}$.

First of all, notice that we may replace $\delta$ by any $0<\delta^{\prime}<\delta$ in equation (3.19), because we can shrink the $\xi_{L}$ and $\xi_{L, w}$ within their homology class to fit in the smaller sets $\mathcal{B}_{L}\left(r_{L}, \delta^{\prime}\right) \backslash \cup_{m \in \mathcal{M}_{L}} L_{m}$. Choose $\delta^{\prime}$ small enough such that for each $L \in \tilde{\mathcal{L}}^{\omega}(k+1) \backslash \tilde{\mathcal{L}}^{\omega}(k)$ there exists a point $\epsilon^{L} \in \mathcal{B}_{r s}^{L}(\epsilon)$ with the property that $\epsilon^{L} T_{u}^{L} \subset U^{L}\left(\delta^{\prime}\right)$.

The singularities of the inner integral are located at codimension 1 cosets in $T^{L}$ of the form $r_{L}^{-1} N$, where $N$ is a connected component of $L \cap L_{m}$ for some $m \in \mathcal{M}^{L}$. We have $r_{L}^{-1} N=r_{L}^{-1} r_{N} T^{N} \subset T^{L}$, and thus $c_{L}^{-1} c_{N} T_{r s}^{N} \subset T_{r s}^{L}$. Choose paths inside $T_{r s}^{L}$ from $w \in \Omega_{L}$ to the point $\epsilon^{L}$. We choose each path such that it intersects the real cosets $c_{L}^{-1} c_{N} T_{r s}^{N}$ transversally and in at most one point, and such that these intersection points are distinct. If $p=\gamma\left(x_{0}\right)$ is the intersection point with the path $\gamma$ from $w \in \Omega_{L}$ to $\epsilon^{L}$ then $p$ is of the form $p=c_{L}^{-1} c_{N} w_{L, N, w} \in c_{L}^{-1} c_{N} T_{r s}^{N}$ with $w_{L, N, w} \in T_{r s}^{N}$. Given $N \in \tilde{\mathcal{L}}^{\omega}(k+2) \backslash \tilde{\mathcal{L}}^{\omega}(k+1)$ we denote by $\Omega_{N}$ the set of all $w_{L, N, w}$ arising in this way, for all the $L \in \tilde{\mathcal{L}}^{\omega}(k+1) \backslash \tilde{\mathcal{L}}^{\omega}(k)$ such that $L \supset N$, and $w \in \Omega_{L}$.

Notice that if $v=w_{L, N, w} \in \Omega_{N}$ and $v s \in r_{N}^{-1}\left(N \cap L_{m}\right)$ for some $m \in \mathcal{M}^{N}$ and $s \in T_{u}$, we have that $c_{L}^{-1} c_{N} v \in c_{L}^{-1}\left(c_{N} T_{r s}^{N} \cap c_{N^{\prime}} T_{r s}^{N^{\prime}}\right)$ where $N^{\prime}=L \cap L_{m}$. Since $T^{N^{\prime}} \neq T^{N}$, this contradicts the assertion that the intersection points of the paths in $T_{r s}^{L}$ and the $\operatorname{cosets} c_{L}^{-1} c_{N} T_{r s}^{N}$ are distinct. We conclude in particular that the compact set $\Omega_{N} T_{u}^{N}$ is contained in the union of the open sets $U^{N}\left(\delta^{\prime}\right)$. We can thus choose $\delta^{\prime}$ small enough such that in fact $\Omega_{N} T_{u}^{N} \subset U^{N}\left(\delta^{\prime}\right)$, as required in equation (3.19). 
Write $T_{N \subset L}$ for the identity component of the 1-dimensional intersection $T_{N} \cap T^{L}$, and decompose the torus $T^{L}$ as the product $T^{N} \times T_{N \subset L}$. Let $v=w_{L, N, w} \in \Omega_{N}$ and put $p=c_{L}^{-1} c_{N} v$ for the corresponding intersection point in $T_{r s}^{L}$. Notice that for a codimension 1 coset $r_{L}^{-1} N^{\prime} \subset T^{L}$ with $N^{\prime} \in \tilde{\mathcal{L}}^{\omega}$ we have that

$$
p T_{N \subset L, u} \cap r_{L}^{-1} N^{\prime}=\left\{\begin{array}{l}
\emptyset \text { if } c_{L}^{-1} c_{N^{\prime}} T^{N^{\prime}} \neq c_{L}^{-1} c_{N} T^{N}, \\
G_{L, N^{\prime}, w} \text { otherwise }
\end{array}\right.
$$

where $G_{L, N^{\prime}, w}$ is a coset of the subgroup $T_{N \subset L} \cap T^{N}$ of the finite group $K_{N^{\prime}}=K_{N}=T_{N} \cap T^{N} \subset T_{u}^{N}$, of the form

$$
G_{L, N^{\prime}, w}=\left(T_{N \subset L} \cap T^{N}\right) r_{L}^{-1} r_{N^{\prime}} v .
$$

The cosets $G_{L, N^{\prime}, w}$ are disjoint. Let $\delta(L, w)$ be the minimum distance of two points in the union of these cosets, and let $\delta(k+1)$ denote the minimum of the positive real numbers $\delta(L, w)$ when we vary over all the $L$ and $w \in \Omega_{L}$. Choose $\delta_{1}>0$ smaller than the minimum of $\delta^{\prime}$ and $\delta(k+1)$. Let $\eta$ be a circle of radius $\delta_{1} / 2$ with center $e$ in $T_{N \subset L}$. Next we make $\delta^{\prime}$ sufficiently small so that $\cup_{N^{\prime}} G_{L, N^{\prime}, w} \eta \subset U^{L}\left(\delta^{\prime}\right)$. For $x_{-}, x_{+}$ suitably close to $x_{0}$ with $x_{-}<x_{0}<x_{+}$we have in $U^{L}\left(\delta^{\prime}\right)$ :

$$
\gamma\left(x_{-}\right) T_{N \subset L, u} \sim \gamma\left(x_{+}\right) T_{N \subset L, u} \cup \cup_{N^{\prime}} G_{L, N^{\prime}, w} \eta,
$$

where the union is over all $N^{\prime} \subset L$ such that $c_{L}^{-1} c_{N^{\prime}} T^{N^{\prime}}=c_{L}^{-1} c_{N} T^{N}$. Define

$$
\xi_{L, N^{\prime}, v}:=r_{L}^{-1} r_{N^{\prime}} \eta \times \xi_{L, w} .
$$

Observe that $T_{N \subset L, u} \times T_{u}^{N}$ is a $\left|T_{N \subset L} \cap T^{N}\right|$-fold covering of $T_{u}^{L}$, and that $g \eta \times v T_{u}^{N} \sim g^{\prime} \eta \times v T_{u}^{N}$ if $g, g^{\prime} \in G_{L, N^{\prime}, w}$. We thus have

$$
\gamma\left(x_{-}\right) T_{u}^{L} \times \xi_{L, w} \sim \gamma\left(x_{+}\right) T_{u}^{L} \times \xi_{L, w} \cup \cup_{N^{\prime}} v T_{u}^{N} \times \xi_{L, N^{\prime}, v} .
$$

By possibly making $\delta^{\prime}$ smaller we get that $\xi_{L, N, v} \subset \mathcal{B}_{N}\left(r_{N}, \delta_{1}\right)$ for all possible choices $N, L$ and $w$. If $L_{m} \supset N$ but $L_{m} \not \supset L$, then, since $r_{L}^{-1} r_{N} \eta \subset U^{L}\left(\delta^{\prime}\right)$ and $\xi_{L, w} \subset \mathcal{B}_{L}\left(r_{L}, \delta^{\prime}\right)$, we have $\xi_{L, N, v} \cap L_{m}=\emptyset$. If on the other hand $L_{m} \supset L$ then $\xi_{L, N, v} \cap L_{m}=r_{L}^{-1} r_{N} \eta \times\left(\xi_{L, w} \cap L_{m}\right)=\emptyset$. Finally we put

$$
\xi_{N, v}:=\cup_{(L, w)} \xi_{L, N, v},
$$

where we take the union over all pairs $(L, w)$ with $L \in \tilde{\mathcal{L}}^{\omega}(k+1) \backslash \tilde{\mathcal{L}}^{\omega}(k)$ such that $L \supset N$ and $w \in \Omega_{L}$ such that there is an intersection point $w_{L, N, w}$ with $w_{L, N, w}=v$. We have shown that

$$
\xi_{N, v} \subset \mathcal{B}_{N}\left(r_{N}, \delta_{1}\right) \backslash \cup_{m \in \mathcal{M}^{N}} L_{m}
$$

as required in equation (3.19). 
Applying equation (3.24) for all the intersections of all the paths we chose, we obtain equation (3.19) with $k$ replaced by $k+1$ and $\delta$ by $\delta_{1}$. We thus take $\xi_{L}=\cup_{w \in \Omega_{L}} \xi_{L, w}$ for $L \in \tilde{\mathcal{L}}^{\omega}(k+1) \backslash \tilde{\mathcal{L}}^{\omega}(k)$, and for $N \in \tilde{\mathcal{L}}^{\omega}(k+2) \backslash \tilde{\mathcal{L}}^{\omega}(k+1)$ we take $\Omega_{N}$ and $\xi_{N, v}$ as constructed above.

This process continues until we have $k=n-1$ in equation (3.19). In the next step we proceed in the same way. Notice that for $N \in$ $\tilde{\mathcal{L}}^{\omega}(n+1) \backslash \tilde{\mathcal{L}}^{\omega}(n)$, either $\Omega_{N}=\{e\}$ (if we cross $c_{L}^{-1} c_{N}$ with some curve from $\Omega_{L}$ to $\epsilon_{L}$ in $T_{r s}^{L}$, for one of the one dimensional residual cosets $L$ containing $N$ ), or else $\Omega_{N}=\emptyset$. The process now stops, since also $\epsilon^{N}=e$. This proves the desired result, with $\delta$ equal to the $\delta_{1}$ obtained in the last step of the inductive construction.

Remark 3.8. The homology classes of the cycles $\xi_{L}$ are not uniquely determined by the above algorithm. The splitting $\mathfrak{X}_{c}=\sum_{\left\{L \mid c_{L}=c\right\}} \mathfrak{X}_{L}$ is not unique without further assumptions. However, in our application to spectral theory of $\mathfrak{C}$, we shall see that the decomposition $\mathfrak{X}_{c}=$ $\sum_{\left\{L \mid c_{L}=c\right\}} \mathfrak{X}_{L}$ is such that each $\mathfrak{X}_{L}$ is a regular measure supported on $L^{\text {temp }}$, and such a decomposition is of course unique.

We list some useful properties of the cycles $\xi_{L}$. We fix $\omega$, and suppress it from the notation.

Definition 3.9. Let $L \in \mathcal{L}$. Denote by $\mathcal{L}^{L}$ the configuration of real cosets $M^{L}:=c_{L} T_{r s}^{M}$ where $M \in \mathcal{L}$ such that $M \supset L, M \neq T$. The "dual" configuration, consisting of the cosets $M_{L}:=c_{L} T_{M, r s} \subset T_{L}$ with $M \in \mathcal{L}$ such that $M \supsetneqq L$, is denoted by $\mathcal{L}_{L}$. Given an (open) chamber $C$ in the complement of $\mathcal{L}^{L}$, we call $C^{d}=\left\{c_{L} \exp (v) \mid(v, w)<0 \forall w \in\right.$

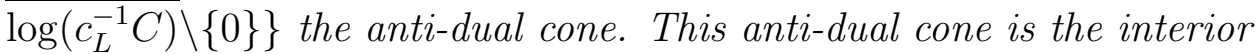
of the closure of a union of chambers of the dual configuration $\mathcal{L}_{L}$ in $T_{L}$. We denote by $\mathcal{L}(L)$ the residual cosets in $T_{L}$ with respect to $K_{L^{-}}$ invariant divisor $\sum_{m \in \mathcal{M}_{L}}\left(L_{m} \cap T_{L}\right)-\sum_{m^{\prime} \in \mathcal{M}_{L}^{\prime}}\left(L_{m^{\prime}} \cap T_{L}\right)$ on $T_{L}$.

Proposition 3.10. (i) If $t_{0}$ is moved inside a chamber of $\mathcal{L}^{L}$ we can leave $\xi_{L}$ unchanged.

(ii) Let $t_{0}(L)=T_{r s}^{L} t_{0} \cap T_{L}$. For each $k \in K_{L}:=T^{L} \cap T_{L}$, we can choose the cycle $\xi_{k r_{L}}(L)$ (defined with respect to the configuration $\mathcal{L}(L)$ in $T_{L}$ and initial point $t_{0}(L) \in T_{L}$ ) equal to $k \xi_{L}$.

Proof. (i) If $t_{0}$ is moved within a chamber of $\mathcal{L}^{L}$, the path from $t_{0}$ to $e$ can be chosen equal to the original path up to a path which only crosses codimension one cosets of the form $L_{m} T_{u} \cap T_{r s}$ which do not contain $c=c_{L}$. Therefore this does not change $\xi_{L}$. 
(ii) We may replace $\mathcal{M}$ by $\mathcal{M}_{L}$ and $\mathcal{M}^{\prime}$ by $\mathcal{M}_{L}^{\prime}$ and leave $\xi_{L}$ is unchanged, because the $L_{m} \not \supset L$ do not contribute to $\xi_{L}$ in the procedure of the proof of Proposition 3.7. By (i) we may also replace $t_{0}$ by $t_{0}(L)$ without changing $\xi_{L}$. We apply Proposition 3.7 in this situation in $T$. Then we intersect with $T_{L}$ and use the formula $T_{L} \cap\left(T_{u}^{L} \times \xi_{L}\right)=$ $\sum_{k \in K_{L}} k \xi_{L}$.

Proposition 3.11. Write $L=r_{L} T^{L}=c_{L} s_{L} T^{L}$ as usual, and let $M \in$ $\mathcal{L}$. Then $L^{\text {temp }} \subset M^{\text {temp }}$ if and only if $L \subset M$ and $e \in M_{L}$. In particular, $L^{\text {temp }}$ is maximal in the collection of $\omega$-tempered cosets if and only if e is regular with respect to the configuration $\mathcal{L}_{L}$.

Proof. If $M \in \mathcal{L}$, then $L^{\text {temp }} \subset M^{\text {temp }} \Leftrightarrow L \subset M$ and $c_{L}=c_{M}$ (since then $s_{L} \in\left(c_{M}^{-1} M\right) \cap T_{u}=s_{M} T_{u}^{M}$, implying that $\left.r_{L} \in M^{\text {temp }}\right)$. Now $c_{L}=c_{M} \Leftrightarrow c_{L} \in T_{M} \Leftrightarrow e \in M_{L}$.

Proposition 3.12. (cf. [18], Lemma 3.3.) If e is not in the closure of the anti-dual cone of the chamber of $\mathcal{L}^{L}$ in which $t_{0}$ lies, we can take $\xi_{L}=\emptyset$.

Proof. By Proposition 3.10 it is sufficient to show this in the case where $L=r_{L}$ is a residual point.

We identify $T_{r s}$ with the real vector space $\mathfrak{t}$ via the map $t \rightarrow \log \left(c_{L}^{-1} t\right)$, and we denote by $\langle\cdot, \cdot\rangle$ the Euclidean inner product thus obtained on $T_{r s}$. Notice that the role of the origin is played by $c_{L}$. The sets $M^{L}=$ $c_{M} T_{r s}^{M}$ with $M \in \mathcal{L}(L)$ satisfy $c_{M} T_{r s}^{M} \ni c_{L}$, and are equipped with the induced Euclidean inner product.

By the assumption and Proposition 3.10 we can choose $t_{0}$ within its chamber such that $\left\langle t_{0}, e\right\rangle>0$. Assume by induction that in the $k$-th step of the inductive process of Proposition 3.7 we have, $\forall N \in \mathcal{L}(L)$ with $\operatorname{codim}(N)=k$ and $\forall w \in \Omega_{N}$, that

$$
\left\langle c_{N} w, c_{N}\right\rangle>0
$$

(see equation (3.19) for the meaning of $\Omega_{N}$ ). Notice in particular that this implies that $\Omega_{N}=\emptyset$ if $c_{N}=c_{L}$. By choosing $\epsilon$ sufficiently small, we therefore have $\left\langle c_{N} \epsilon^{N}, c_{N}\right\rangle>0$ when $\Omega_{N} \neq \emptyset$. Take the path $\gamma$ in $T_{r s}^{N}$ from $w \in \Omega_{N}$ to $\epsilon^{N}$ equal to $c_{N}^{-1}\left[c_{N} w, c_{N} \epsilon^{N}\right]$, where $\left[c_{N} w, c_{N} \epsilon^{N}\right]$ denotes the (geodesic) segment from $c_{N} w$ to $c_{N} \epsilon^{N}$ in the Euclidean space $c_{N} T_{r s}^{N}$. Consequently, we have $\left\langle x, c_{N}\right\rangle>0$ for all $x \in\left[c_{N} w, c_{N} \epsilon^{N}\right]$. Let $M \subset$ $\mathcal{L}(L)$ with $\operatorname{codim}(M)=k+1$ and $M \subset N$. If $\gamma$ intersects $c_{N}^{-1} c_{M} T_{r s}^{M}$ in $c_{N}^{-1} c_{M} w_{N, M, w}$, then we have $0<\left\langle c_{M} w_{N, M, w}, c_{N}\right\rangle=\left\langle c_{M} w_{N, M, w}, c_{M}\right\rangle$. By induction on $k$ this proves that we can perform the contour shifts in such a way that (3.27) holds for each $k \in\{0, \ldots, \operatorname{codim}(L)\}$. This implies that $\Omega_{L}=\emptyset$, and thus that $\xi_{L}=\emptyset$. 
In the next proposition we view the constants $d_{m}$ as variables. We choose a continuous path $[0,1] \ni \sigma \rightarrow\left(d_{m}(\sigma)\right)_{m \in \mathcal{M}}$ from $\left(d_{m}\right)_{m \in \mathcal{M}}$ to $\left(d_{m}^{\prime}\right)_{m \in \mathcal{M}}$, and consider the resulting deformation of $\omega$ and $\mathcal{L}$. The end point of the path corresponds to the form $\omega^{\prime}$ and its collection of $\omega^{\prime}$ residual cosets, denoted by $\mathcal{L}^{\prime}$. Recall that $\mathcal{M}_{L}$ denotes the multiset of $m \in \mathcal{M}$ such that $L_{m} \supset L$. Assume that $\cap_{m \in \mathcal{M}_{L}} L_{m}(\sigma) \neq \emptyset$ for all $\sigma$. In this situation there exists a continuous path $\sigma \rightarrow r_{L}(\sigma)$ such that $L(\sigma):=r_{L}(\sigma) T^{L}$ is a connected component of $\cap_{m \in \mathcal{M}_{L}} L_{m}(\sigma)$. We may take $r_{L}(\sigma) \in T_{L} \cap L(\sigma)$. We put $L^{\prime}=L(1)$. Assume that $\left\{m \in \mathcal{M} \mid x_{m}\left(L^{\prime}\right)=d_{m}^{\prime}\right\}=\left\{m \in \mathcal{M} \mid x_{m}(L)=d_{m}\right\}$.

Proposition 3.13. Assume that $e(\sigma):=c_{L} c_{L(\sigma)}^{-1}$ stays within a facet of $\mathcal{L}_{L}$ for all $\sigma$, and $t_{0}(\sigma):=t_{0} c_{L} c_{L(\sigma)}^{-1}$ stays within a chamber of $\mathcal{L}^{L}$. With these assumptions we can take $\xi_{L^{\prime}}=r_{L}^{-1} r_{L^{\prime}} \xi_{L}$.

Proof. As above, we may assume that in fact $L=r_{L}$ is a point. The only contributions to $\xi_{L}$ come from contour shifts inside residual cosets of the configuration $\mathcal{L}(L)$ as in the proof of Proposition 3.12. Likewise, for the construction of $\xi_{L^{\prime}}$ we only need to consider the translated configuration $r_{L}^{-1} r_{L^{\prime}} \mathcal{L}(L)$. By the assumption on $t_{0}$ and Proposition 3.10 we can construct $r_{L} r_{L^{\prime}}^{-1} \xi_{L^{\prime}}$ by working with $\mathcal{L}(L)$ and $t_{0}$, but with the center $e$ of $T$ replaced by $e^{\prime}:=e(1)$.

We now follow the deformations of the centers $c_{M}(\sigma)$ with $\sigma \in[0,1]$ and $M \in \mathcal{L}(L)$. The assumption on $e(\sigma)$ implies that $c_{M} \neq c_{L} \Leftrightarrow \forall \sigma$ : $c_{M}(\sigma) \neq c_{L}$. This implies we can use $\xi_{L}$ also as the cycle associated with $L$ relative to the center $e^{\prime}$.

Remark 3.14. Note that for some $\sigma \in(0,1)$ there may be additional $M \in \mathcal{L}(\sigma)$ such that $L(\sigma) \subset M$. It may also happen that for some values of $\sigma \in[0,1], L(\sigma)^{\text {temp }}$ contains smaller tempered cosets. We may need to adjust $\epsilon^{L(\sigma)}$ accordingly.

\subsection{Application to the trace functional}

We will now apply the above results to the integral (3.1). We thus use the rational $(n, 0)$-form

$$
\eta(t):=\frac{d t}{q\left(w_{0}\right)^{2} \Delta(t) c(t, q) c\left(t^{-1}, q\right)}
$$

and define the notion of quasi-residual coset as the $\eta$-residual cosets introduced above. We write $\mathcal{L}^{\mathrm{qu}}$ for the collection of these $\eta$-residual spaces, $\mathcal{C}^{\mathrm{qu}}$ for their centers etc. Note: the collection of residual cosets of Appendix 7 is strictly included in this collection. 
Apply Lemma 3.4 to $\eta$ of equation (3.28), with $t_{0}$ such that (3.7) is satisfied. Denote the resulting local distributions by $\mathfrak{X}_{\eta, c}$.

Proposition 3.15. The collection $\left\{\mathfrak{X}_{c}^{h}\right\}_{c \in \mathcal{C}^{\mathrm{qu}}, h \in \mathcal{H}}$ of distributions $\mathfrak{X}_{c}^{h} \in$ $C^{-\infty}\left(c T_{u}\right)$ defined by $\mathfrak{X}_{c}^{h}(a):=\mathfrak{X}_{\eta, c}\left(\left\{t \rightarrow a(t) E_{t}(h)\right\}\right)$ satisfies

(i) $\operatorname{supp}\left(\mathfrak{X}_{c}^{h}\right) \subset S_{c}^{\text {qu }}$.

(ii) $\forall a \in \mathcal{A}: \tau(a h)=\sum_{c \in \mathcal{C} \text { qu }} \mathfrak{X}_{c}^{h}(a)$ (where $\mathfrak{X}_{c}(a)$ means $\mathfrak{X}_{c}\left(\left.a\right|_{c T_{u}}\right)$ ).

(iii) The application $h \rightarrow \mathfrak{X}_{c}^{h}$ is $\mathbb{C}$-linear.

(iv) $\forall a, b \in \mathcal{A}, h \in \mathcal{H}: \mathfrak{X}_{c}^{a h}(b)=\mathfrak{X}_{c}^{h}(a b)$.

Proof. These properties are simple consequences of 3.9 .

3.3.1. Symmetrization and positivity. The main objects of this section are the $W_{0}$-symmetric versions of the local distributions $\mathfrak{X}_{c}^{h}$.

Definition 3.16. Let $\mathcal{C}_{-}^{\mathrm{qu}}$ denote the set of elements in $\mathcal{C}^{\mathrm{qu}}$ which lie in the closure of the negative chamber $T_{r s,-}=\left\{t \in T_{r s} \mid \forall \alpha \in R_{0,+}\right.$ : $\alpha(t)<1\}$. For $h \in \mathcal{H}, a \in \mathcal{A}$, and $c \in \mathcal{C}_{-}^{\mathrm{qu}}$ put:

$$
\mathfrak{Y}_{c}^{h}(a):=\sum_{c^{\prime} \in W_{0} c} \mathfrak{X}_{c^{\prime}}^{h}(\bar{a}),
$$

where $\bar{a}:=\left|W_{0}\right|^{-1} \sum_{w \in W_{0}} a^{w}$. Then $\mathfrak{Y}_{c}^{h}$ is a $W_{0}$-invariant distribution on $\cup_{c^{\prime} \in W_{0} c} c^{\prime} T_{u}$, with support in $W_{0} S_{c}^{\mathrm{qu}}$, such that for all $z \in \mathcal{Z}$ :

$$
\tau(z h)=\sum_{c \in \mathcal{C}_{-}^{\mathrm{qu}}} \mathfrak{Y}_{c}^{h}(z) .
$$

It is elementary to compute the distribution $\mathfrak{Y}_{c}^{h}$ when $c=e$. Recall that ([37], Corollary 2.26) we have the following identity for the character of the minimal principal series $I_{t}$ :

$$
\chi_{I_{t}}=q\left(w_{0}\right)^{-1} \sum_{w \in W_{0}} \Delta(w t)^{-1} E_{w t} .
$$

Hence we can write for all $z \in \mathcal{Z}$ :

$$
\begin{aligned}
\mathfrak{Y}_{e}^{h}(z) & =\int_{T_{u}} z(t) E_{t}(h) \eta(t) \\
& =\int_{W_{0} \backslash T_{u}} z(t) \chi_{I_{t}}(h) d \mu_{T}(t),
\end{aligned}
$$

where $\mu_{T}$ is the positive measure on $T_{u}$ given by

$$
d \mu_{T}(t):=\frac{d t}{q\left(w_{0}\right) c(t) c\left(t^{-1}\right)} .
$$


Here we used the $W_{0}$-invariance of $c(t) c\left(t^{-1}\right)$, and the fact that for $t \in T_{u}$ we have

$$
c(t) c\left(t^{-1}\right)=c(t) c(\bar{t})=|c(t)|^{2} .
$$

We see that $h \rightarrow \mathfrak{Y}_{e}^{h}(1)$ is the integral of the function $T_{u}=S_{e} \ni t \rightarrow$ $\chi_{I_{t}}(h)$ against a positive measure on $T_{u}$. Moreover, for every $t \in T_{u}$, the function $h \rightarrow \chi_{I_{t}}(h)$ is positive and central, and is a $\mathcal{Z}$-eigenfunction with character $t$. Our first task will be to prove these properties for arbitrary centers $c \in \mathcal{C}^{\mathrm{qu}}$. The main tools we will employ are the approximating sequences.

3.3.2. Positivity and centrality of the kernel. Let us choose, for a suitably large $N$, approximating sequences $a_{n}^{c}$ for the distributions $\mathfrak{X}_{\eta, c}$. We remark that the group $\pm W_{0}$ acts on the collection of quasi-residual subspaces. In addition, complex conjugation also leaves this collection stable. We define an action - on $\mathcal{A}$ of the group $G$ of automorphisms of $T$ generated by $W_{0}$, inv $: t \rightarrow t^{-1}$ and conj $: t \rightarrow \bar{t}$. For elements $g \in \pm W_{0}$ this action is given by $g \cdot a:=a^{g}$, and $(\operatorname{conj} \cdot a)(t):=\overline{a(\bar{t})}$.

Lemma 3.17. We can choose the $a_{n}^{c}$ in a G-equivariant way, i.e. such that $\forall g \in G: a_{n}^{g c}=g \cdot\left(a_{n}^{c}\right)$.

Proof. Just notice that for any given collection of approximating sequences $A:=\left\{a_{n}^{c}\right\}$ and any $g \in G, g \cdot A=\left\{g \cdot a_{n}^{g^{-1} c}\right\}$ is also a collection of approximating sequences for the distributions $\mathfrak{X}_{\eta, c}$, and this defines an action of $G$ on the set of collections of approximating sequences for the $\mathfrak{X}_{\eta, c}$. Hence we can take the average over $G$.

For $c \in \mathcal{C}_{-}^{\text {qu }}$ we now define

$$
z_{n}^{c}:=\sum_{c^{\prime} \in W_{0} c} a_{n}^{c^{\prime}}
$$

Then these sequences in the center $\mathcal{Z}$ of $\mathcal{H}$ have the property that for all $c \in \mathcal{C}_{-}^{\text {qu }}, z \in \mathcal{Z}$ and $h \in \mathcal{H}$ :

$$
\mathfrak{Y}_{c}^{h}(z)=\lim _{n \rightarrow \infty} \tau\left(z_{n}^{c} z h\right) .
$$

It is easy to see that the map $h \rightarrow \mathfrak{Y}_{c}^{h}$ is central:

Proposition 3.18. For all $c \in \mathcal{C}_{-}^{\mathrm{qu}}$, we have $\mathfrak{Y}_{c}^{h}=0$ if $h$ is a commutator.

Proof. We compute $\mathfrak{Y}_{c}^{h}(z)=\lim _{n \rightarrow \infty} \tau\left(z_{n}^{c} z h\right)=0$, because $z_{n}^{c} z h$ is also a commutator and $\tau$ is central. 
We define an anti-holomorphic involutive map $t \rightarrow t^{*}$ on $T$ by $t^{*}:=$ $\overline{t^{-1}}$. In view of the action of conjugation on $\mathcal{A}$, we see that for all $z \in \mathcal{Z}, z^{*}(t)=\overline{z\left(t^{*}\right)}$. By Lemma 3.17 we have, for each $c \in \mathcal{C}_{-}^{\mathrm{qu}}$,

$$
z_{n}^{c^{*}}(t)=z_{n}^{c}\left(t^{-1}\right)=\left(z_{n}^{c}\right)^{*}(t) .
$$

Now we embark on the proof that the distributions $\mathfrak{Y}_{c}^{h}$ are in fact (complex) measures.

Lemma 3.19. (i) If $c^{*} \notin W_{0} c$ then $\mathfrak{Y}_{c}^{h}=0$.

(ii) Let $c^{*} \in W_{0} c$, and $c s \in S_{c}^{\text {qu }}$ such that $(c s)^{*}=c^{-1} s \notin W_{0}(c s)$. Then cs $\notin \operatorname{Supp}\left(\mathfrak{Y}_{c}^{h}\right)$.

Proof. (i). Any $h \in \mathcal{H}$ can be decomposed as $h=h_{r}+i h_{i}$ with $h_{r}^{*}=h_{r}$ and $h_{i}^{*}=h_{i}$, so it suffices to prove the assertion for $h \in \mathcal{H}^{r e}$. Thus by Lemma 2.15 it is sufficient to prove the assertion for a positive element $h \in \mathcal{H}_{+}$. Similarly $z \in \mathcal{Z}$ is a linear combination of positive central elements, so that it is sufficient to show that $\mathfrak{Y}_{c}^{h}(z)=\mathfrak{Y}_{c}^{z h}(1)=0$ for each positive central element $z$. By Lemma 2.15 this reduces our task to proving that $\mathfrak{Y}_{c}^{h}(1)=0$ for an arbitrary element $h \in \mathcal{H}_{+}$. Then

$$
0 \leq \lim _{n \rightarrow \infty} \tau\left(h\left(z_{n}^{c^{*}}+u z_{n}^{c}\right)^{*}\left(z_{n}^{c^{*}}+u z_{n}^{c}\right)\right)=u \mathfrak{Y}_{c}^{h}(1)+\bar{u} \mathfrak{Y}_{c^{*}}^{h}(1)
$$

It follows easily that $\mathfrak{Y}_{c^{*}}^{h}(1)=\mathfrak{Y}_{c}^{h}(1)=0$.

(ii). This is essentially the same argument that we used to prove (i). Since $(c s)^{*} \notin W_{0}(c s)$, we can find an open neighborhood $U \ni c s$ in $c T_{u}$ such that $W_{0} U \cap U^{*}=\emptyset$. Let $\phi \in C_{c}^{\infty}\left(W_{0} U\right)^{W_{0}}$. Then $\phi^{*} \phi=0$, where $\phi^{*}(x):=\overline{\phi\left(x^{*}\right)}$. We want to prove that $\mathfrak{Y}_{c}^{h}(\phi)=0$ for $h \in \mathcal{H}_{+}$. By Fourier analysis on $c T_{u}$ we can find a sequence $f_{n} \in \mathcal{A}^{W_{c}}$ such that $D\left(f_{n}\right)$ converges uniformly to $D(\phi)$ on $c T_{u}$ for every holomorphic constant coefficient differential operator $D$ on $T$ of order at most $N$ on $T$. We can then find a sequence $g_{n}$ of the form $g_{n}=f_{n} a_{k(n)}^{c}$ such that $D\left(g_{n}\right)$ converges uniformly to $D(\phi)$ on $S_{c}^{\text {qu }}$, and to 0 on $S_{c^{\prime}}^{\text {qu }}$ for every $c^{\prime} \neq c$. Hence if we put

$$
\phi_{n}=\sum_{w \in W^{c}} g_{n}^{w} \in \mathcal{Z}
$$

then for each holomorphic constant coefficient differential operator $D$ on $T$ of order at most $N, D\left(\phi_{n}\right) \rightarrow D(\phi)$ uniformly on $W_{0} S_{c}^{\text {qu }}$, and $D\left(\phi_{n}\right) \rightarrow 0$ uniformly on $S_{c^{\prime}}^{\text {qu }}$ for $c^{\prime} \notin W_{0} c$. Hence $\forall h \in \mathcal{H}_{+}, u \in \mathbb{C}$,

$$
\begin{aligned}
0 & \leq \lim _{n \rightarrow \infty} \tau\left(h\left(u z_{n}^{c}+\phi_{n}\right)^{*}\left(u z_{n}^{c}+\phi_{n}\right)\right) \\
& =|u|^{2} \mathfrak{Y}_{c}^{h}(1)+u \mathfrak{Y}_{c}^{h}\left(\phi^{*}\right)+\bar{u} \mathfrak{Y}_{c}^{h}(\phi)
\end{aligned}
$$


If we divide this inequality by $|u|$ and send $|u|$ to 0 , we get that $\forall \epsilon \in \mathbb{C}$ with $|\epsilon|=1$,

$$
0 \leq \epsilon \mathfrak{Y}_{c}^{h}\left(\phi^{*}\right)+\bar{\epsilon} \mathfrak{Y}_{c}^{h}(\phi)
$$

It follows that $\forall h \in \mathcal{H}_{+}, \mathfrak{Y}_{c}^{h}(\phi)=\mathfrak{Y}_{c}^{h}\left(\phi^{*}\right)=0$. Hence the same is true for arbitrary $h \in \mathcal{H}$.

Corollary 3.20. If $h \in \mathcal{H}_{+}$, the distribution $\mathfrak{Y}_{c}^{h}$ is a $W_{0}$-invariant positive Radon measure on $W_{0} c T_{u}$, supported on $W_{0} S_{c}^{\mathrm{qu}}$.

Proof. It suffices to show that $\mathfrak{Y}_{c}^{h}$ is a positive distribution. Assume that $\phi \in C^{\infty}\left(W_{0} c T_{u}\right)^{W_{0}}$ and that $\phi>0$. Then the positive square root $\sqrt{\phi}$ is also in $C^{\infty}\left(W_{0} c T_{u}\right)^{W_{0}}$. Using the approximating sequences as we did before, we can find a sequence $f_{n} \in \mathcal{Z}$ such that $D\left(f_{n}\right) \rightarrow D(\sqrt{\phi})$, uniformly on $W_{0} S_{c}^{\text {qu }}$, and to 0 on $S_{c^{\prime}}^{\text {qu }}$ for $c^{\prime} \neq c$. By Lemma 3.19, the support of $\mathfrak{Y}_{c}^{h}$ is contained in $W_{0} S_{c}^{\text {herm }}:=W_{0} S_{c}^{\text {qu }} \cap T^{\text {herm }}$, where $T^{\text {herm }}:=\left\{t \in T \mid t^{*} \in W_{0} t\right\}$. This is itself a regular support for distributions. On $W_{0} S_{c}^{\text {herm }}$, the sequence $\phi_{n}:=f_{n}^{*} f_{n} \in \mathcal{Z}_{+}$converges uniformly to $\phi$ up to derivatives of order $N$. Hence

$$
0 \leq \lim _{n \rightarrow \infty} \tau\left(h f_{n}^{*} f_{n}\right)=\mathfrak{Y}_{c}^{h}(\phi)
$$

This proves the desired inequality.

Corollary 3.21. Put $\nu_{c}:=\mathfrak{Y}_{c}^{1}$. This is a positive Radon measure, with support in $W_{0} S_{c}^{\mathrm{qu}}$, and for all $h \in \mathcal{H}, \mathfrak{Y}_{c}^{h}$ is absolutely continuous with respect to $\nu_{c}$.

Proof. It is enough to prove this for $h \in \mathcal{H}$ which are Hermitian, i.e. such that $h^{*}=h$. By Lemma 2.15 and Corollary 3.20 we see that for positive functions $\phi \in C^{\infty}\left(W_{0} c T_{u}\right)^{W_{0}}$,

$$
-\|h\|_{o} \nu_{c}(\phi) \leq \mathfrak{Y}_{c}^{h}(\phi) \leq\|h\|_{o} \nu_{c}(\phi) .
$$

Definition 3.22. Let $\nu:=\sum_{c \in \mathcal{C}^{\mathrm{qu}}} \nu_{c}$. By equation (3.30), this is the spectral measure on $\hat{\mathcal{Z}}$ of the restriction of $\tau$ to $\mathcal{Z}$ (the "Plancherel measure" of $\mathcal{Z})$. For $h \in \mathcal{H}$ we define a measurable, essentially bounded, $W_{0}$-invariant function $t \rightarrow \chi_{t}(h)$ on $T$ by

$$
\sum_{c \in \mathcal{C}_{-}^{q u}} \mathfrak{Y}_{c}^{h}\left(\left.\phi\right|_{W_{0} S_{c}^{\mathrm{qu}}}\right)=\int_{T} \phi(t) \chi_{t}(h) d \nu(t)
$$

for each $\phi \in C_{c}(T)^{W_{0}}$. For $t$ outside the support of $\nu$ we set $\chi_{t}(h)=0$. 
ON THE SPECTRAL DECOMPOSITION OF AFFINE HECKE ALGEBRAS 47

Corollary 3.23. The function $t \rightarrow \chi_{t} \in \mathcal{H}^{*}$ satisfies

(i) The support of $t \rightarrow \chi_{t}$ is the support of $\nu$.

(ii) $\chi_{t} \in \mathcal{H}^{*}$ is a positive, central functional such that $\chi_{t}(1)=1$, $\nu$ almost everywhere on $T$.

(iii) For $h \in \mathcal{H}, z \in \mathcal{Z}: \chi_{t}(z h)=z(t) \chi_{t}(h)$, $\nu$ almost everywhere on $T$.

(iv) $\chi_{t}$ extends, for $\nu$-almost all $t$, to a continuous tracial state of the $C^{*}$-algebra $\mathfrak{C}$.

(v) We have the following decomposition of $\tau$ for all $h \in \mathcal{H}$,

$$
\tau(h)=\int_{T} \chi_{t}(h) d \nu(t)
$$

Proof. Everything is clear. Assertion (iv) follows from Corollary 2.17 by (ii).

For $t \in \operatorname{Supp}(\nu)$, we define the positive semi-definite Hermitian form $(x, y)_{t}:=\chi_{t}\left(x^{*} y\right)$ associated to the tracial state $\chi_{t}$ of $\mathcal{H}$. It is clear that the maximal ideal $\mathcal{I}_{t} \subset \mathcal{Z}$ of elements vanishing at $t$ is contained in the radical $\operatorname{Rad}_{t}$ of $(\cdot, \cdot)_{t}$. Hence the radical is a cofinite two-sided ideal of $\mathcal{H}$. Consequently the GNS-construction produces a finite dimensional Hilbert algebra associated with $\chi_{t}$ :

Definition 3.24. The algebra $\overline{\mathcal{H}^{t}}:=\mathcal{H} / \operatorname{Rad}_{t}$ is a finite dimensional Hilbert algebra with trace $\chi_{t}$. We will refer to this Hilbert algebra as the residual algebra at $t$.

Let $\left\{e_{i}\right\}_{i=1}^{l_{t}}$ denote the set of minimal central idempotents of $\overline{\mathcal{H}^{t}}$, and $\chi_{t, i}$ the associated irreducible characters given by

$$
\chi_{t, i}(x)=\operatorname{dim}\left(e_{i} \overline{\mathcal{H}^{t}}\right)^{1 / 2} \chi_{t}\left(e_{i}\right)^{-1} \chi_{t}\left(e_{i} x\right)
$$

We define $d_{t, i}:=\operatorname{dim}\left(e_{i} \overline{\mathcal{H}^{t}}\right)^{-1 / 2} \chi_{t}\left(e_{i}\right) \in \mathbb{R}_{+}$, so that

$$
\chi_{t}=\sum_{i=1}^{l_{t}} \chi_{t, i} d_{t, i}
$$

Note that everything in sight depends on the orbit $W_{0} t$ rather than on $t$ itself. We will sometimes use the notation $d_{W_{0} t, i}$ etc. in order to stress this. (This notation and parametrization for the irreducible characters of $\overline{\mathcal{H}^{t}}$ is provisional. We return to these matters in a systematic way in Section 4 (see e.g. Theorem 4.23).) 


\subsection{The Plancherel measure $\nu$ of $\mathcal{Z}$, and the $\mathcal{A}$-weights of $\chi_{t}$}

The results in this subsection are based on the fact that the Eisenstein kernel of (3.1) simplifies considerably when restricted to the subalgebra $\mathcal{A} \supset \mathcal{Z}$ of $\mathcal{H}$. This means that the $(n, 0)$-form $\eta$ (see (3.28)) can be replaced by the better behaved $(n, 0)$-form $\omega$ (cf. (3.2) and subsection 7.1) in the residue calculus. This has as an important consequence (see below) that the support of the measure $\nu$ can be identified as the union of the tempered residual cosets, which is only a small subcollection of the tempered quasi residual cosets, and very well behaved (see Subsection 7.3 of Appendix 7). Since we have derived that $\mathfrak{Y}^{h}$ is absolutely continuous with respect to $\nu$ for general $h \in \mathcal{H}$ (see Corollary 3.21 ), we conclude that the support of the density function $t \rightarrow \chi_{t}$ is the union of the tempered residual cosets.

The probability measure $\nu$ can be computed almost explicitly, due to the good properties of residual cosets. We will exploit these facts here to study the behaviour of the states $\chi_{t}$ on $\mathcal{A}$.

Theorem 3.25. The $W_{0}$-invariant probability measure $\nu$ has a decomposition $\nu=\sum_{L} \nu_{L}$, where $L$ runs over the collection of residual cosets as defined in Appendix 7, and where $\nu_{L}$ is the push forward to $T$ of a smooth measure on $L^{\text {temp }}$. Let $d^{L}$ denote the normalized Haar measure on $T_{u}^{L}$, transported to the coset $L^{\text {temp }}$ by translation. The measure $\nu_{L}$ is given by a density function $\bar{\kappa}_{W_{L} L} m_{L}(t):=\frac{d \nu_{L}(t)}{d^{L} t}$, where $\bar{\kappa}_{W_{L} L} \in \mathbb{Q}$ is a constant, and where $m_{L}$ is of the form

$$
m_{L}(t)=q\left(w_{0}\right) \frac{\prod_{\alpha \in R_{1}}^{\prime}(\alpha(t)-1)}{\prod_{\alpha \in R_{1}}^{\prime}\left(q_{\alpha^{\vee}}^{1 / 2} \alpha(t)^{1 / 2}+1\right) \prod_{\alpha \in R_{1}}^{\prime}\left(q_{\alpha^{\vee}}^{1 / 2} q_{2 \alpha^{\vee}} \alpha(t)^{1 / 2}-1\right)} .
$$

Here we used the convention of Remark 3.1. The constant $\bar{\kappa}_{W_{L} L}$ is independent of $\mathbf{q}$ if we assume $q$ to be as in Convention 2.1. The notation $\prod^{\prime}$ means that we omit the factors which are identically equal to 0 on $L$. The density $m_{L}$ is a smooth function on $L^{\text {temp }}$.

Proof. We know already that $\nu$ is a $W_{0}$-invariant measure supported on the union of the tempered quasi residual cosets. We apply Proposition 3.7 to the integral

$$
\tau(a)=\int_{t_{0} T_{u}} a \omega=\sum_{c \in \mathcal{C}^{\mathrm{qu}}} \mathfrak{X}_{c}^{1}(a)
$$

(cf. equations (3.1), (3.2) and 3.9). Choose $\epsilon>0$. For a suitably small $\delta>0$ we can find, for each quasi residual subspace $L$, an $\epsilon^{L} \in T_{\text {rs }}^{L}$ in an $\epsilon$ neighborhood of $e$, and a cycle $\xi_{L} \subset \mathcal{B}_{L}\left(r_{L}, \delta\right) \backslash \cup_{L_{m}^{\prime} \supset L} L_{m}^{\prime}$, where 
ON THE SPECTRAL DECOMPOSITION OF AFFINE HECKE ALGEBRAS 49

$\mathcal{B}_{L}\left(r_{L}, \delta\right) \subset T_{L}$ denotes a ball of radius $\delta>0$ centered around $r_{L}$, such that

$$
\mathfrak{X}_{c}^{1}(a):=\sum_{L: c_{L}=c} k_{L} \int_{t \in \epsilon^{L} T_{u}^{L}}\left\{\int_{\xi_{L}} a\left(t t^{\prime}\right) \frac{d_{L}\left(t^{\prime}\right)}{q\left(w_{0}\right) c\left(t t^{\prime}\right) c\left(\left(t t^{\prime}\right)^{-1}\right)}\right\} d^{L}(t) .
$$

Here $d^{L}(t)$ is the holomorphic extension to $L$ of $d^{L}$, and $d_{L} t^{\prime}$ denotes the Haar measure on $T_{L, u}$, also extended as a holomorphic form on $T_{L}$. We assume that $\delta$ is small enough to assure that $\log$ is well defined on $\mathcal{B}_{L}\left(r_{L}, \delta\right)$. For the inner integral we use a basis $\left(x_{i}\right)$ of $X \cap \mathbb{Q} R_{L}$ as coordinates on $\log \left(\mathcal{B}_{L}\left(r_{L}, \delta\right)\right)$, shifted so that the coordinates are centered at $\log \left(r_{L}\right)$. We can then write the integration kernel as:

$$
t^{\prime} \rightarrow a\left(t t^{\prime}\right) m_{L}\left(t t^{\prime}\right)\left(1+f_{t}\left(t^{\prime}\right)\right) \omega_{L}\left(t^{\prime}\right)
$$

where $\omega_{L}$ is a rational homogeneous $\left(l:=\operatorname{dim}\left(T_{L}\right), 0\right)$-form (independent of $t)$ in the $x_{i}$, and $f_{t}$ is a power series in $x_{i}$ such that $f_{t}(0)=0$. In fact, the form $\omega_{L}$ is easily seen to be (including the factor $k_{L}$ of (3.48))

$$
\omega_{L}(x)=\frac{\prod_{\alpha \in R_{L}^{z}} \alpha(x)}{(2 \pi i)^{l} \prod_{\beta \in R_{L}^{p}} \beta(x)} d x_{1} \wedge d x_{2} \cdots \wedge d x_{l} .
$$

By Corollary 7.12 it follows that the form $\omega_{L}$ has homogeneous degree $\geq 0$ if $L$ is residual in the sense of Definition 7.1. A homogeneous closed rational form of positive homogeneous degree is exact. Hence the inner integral will be nonzero only if $L$ is in fact a residual coset. In that case the inner integral will have value

$$
\kappa_{L} a\left(r_{L} t\right) m_{L}\left(r_{L} t\right)
$$

with

$$
\kappa_{L}=\int_{\xi_{L}} \omega_{L}
$$

We note that $\kappa_{L} \in \mathbb{Q}$, since $\omega_{L}$ defines a rational cohomology class. Let us therefore assume that $L$ is residual from now on. Write $r_{L}=s c$. By Theorem 7.14 we know that $r_{L}^{*}=s c^{-1}=w_{s}\left(r_{L}\right)$ with $w_{s} \in W\left(R_{L, s, 1}\right)$. When $t \in L^{t e m p}$, the expression $m_{L}(t)$ can be rewritten as

$$
q\left(w^{L}\right) m_{\mathcal{R}_{L},\left\{r_{L}\right\}}\left(r_{L}\right) \prod_{\alpha \in R_{1,+} \backslash R_{L, 1,+}} \frac{|1-\alpha(t)|^{2}}{\left|1+q_{\alpha^{\vee}}^{1 / 2} \alpha(t)^{1 / 2}\right|^{2}\left|1-q_{\alpha^{\vee}}^{1 / 2} q_{2 \alpha^{\vee}} \alpha(t)^{1 / 2}\right|^{2}} .
$$

Here we used that if $t=c u \in L^{t e m p}$ with $u \in s T_{u}^{L}$, we have $w_{s} c=c^{-1}$, $w_{s} u=u$, and $w_{s}\left(R_{1,+} \backslash R_{L, 1,+}\right)=R_{1,+} \backslash R_{L, 1,+}$. By the same argument as was used in Theorem 3.13 of [18] we see that this expression is real analytic on $L^{t e m p}$. This implies that we can in fact take $\epsilon^{L}=e$ for all 
residual $L$ in equation (3.48) after we evaluate the inner integrals. This leads to

$$
\mathfrak{X}_{c}^{1}(a)=\sum_{L: c_{L}=c} \kappa_{L} \int_{L^{\text {temp }}} a(t) m_{L}(t) d^{L}(t) .
$$

where the sum is taken over residual cosets only. When we combine terms over $W_{0}$ orbits of residual cosets we find the desired result. Let $W_{0} L$ denote the set of residual cosets in the orbit of $L$. We have to take

$$
\bar{\kappa}_{W_{L} L}=\frac{1}{\left|W_{0} L\right|} \sum_{L^{\prime} \in W_{0} L} \kappa_{L^{\prime}}
$$

When we now define a measure $\nu_{L}$ on $L^{\text {temp }}$ by

$$
\int_{t \in L^{\text {temp }}} f(t) d \nu_{L}(t):=\bar{\kappa}_{W_{L} L} \int_{t^{L} \in T_{u}^{L}} f\left(r_{L} t^{L}\right) m_{L}\left(r_{L} t^{L}\right) d^{L}\left(t^{L}\right)
$$

then we have the equality $\nu=\sum_{L} \nu_{L}$ (sum over the residual subspaces).

We note in addition that $\kappa_{L}=k_{L} \kappa_{\mathcal{R}_{L},\left\{r_{L}\right\}}$, because the cycle $\xi_{L}$ is constructed inside $T_{L}$, entirely in terms of the root system $R_{L}$ (see Proposition 3.10) (the factor $k_{L}$ comes from the facorization $d t=$ $k_{L} d^{L} t^{L} d_{L} t_{L}$, see $\left.(3.48)\right)$. Also, it is clear that $m_{\mathcal{R}_{L},\left\{r_{L}\right\}}\left(r_{L}\right)$ is independent of the choice of $r_{L}$, because the finite group $K_{L}=T_{L} \cap T^{L}$ is contained in the simultaneous kernel of the roots of $R_{L}$. Finally, the independence of $\mathbf{q}$ is clear from Proposition 3.13. When we apply a scaling transformation $\mathbf{q} \rightarrow \mathbf{q}^{\epsilon}$, the point $c_{L}$ moves such that the facet of the dual configuration containing $e$ does not change. Hence $r_{L}^{-1} \xi_{L}$ and $\omega_{L}$ will be independent of $\epsilon$.

Remark 3.26. We note that the smoothness of $m_{L}$ implies Theorem 7.17, similar to [18], Remark 3.14.

Proposition 3.27. For $L$ residual consider the root datum $\mathcal{R}_{L}=$ $\left(X_{L}, Y_{L}, R_{L}, R_{L}^{\vee}, F_{L}\right.$ ) (see Subsection 2.2) associated with the parabolic root subsystem $R_{L} \subset R_{0}$. Let $q_{L}$ be the restriction of the label function $q$ to $\mathcal{R}_{L}$. Then $\left\{r_{L}\right\} \subset T_{L}$ is a $\left(\mathcal{R}_{L}, q_{L}\right)$ residual point. Assume that $R_{L}$ is a standard parabolic subsystem of roots, and thus that $F_{L} \subset F_{0}$. Denote by $W_{L}$ the standard parabolic subgroup $W_{L}=W\left(R_{L}\right)$ of $W_{0}$, and let $W^{L}$ denote the set of minimal length representatives of the left $W_{L}$ cosets in $W_{0}$.

(i) When $w \in W^{L}$, we may take $\xi_{w L}=w\left(\xi_{L}\right)$. Consequently, $\kappa_{L}=\kappa_{w L}$ if $w \in W^{L}$. 
ON THE SPECTRAL DECOMPOSITION OF AFFINE HECKE ALGEBRAS 51

(ii) Put

$$
\begin{aligned}
m^{L}(t) & =q\left(w^{L}\right)^{-1} \prod_{\alpha \in R_{1} \backslash R_{L, 1}} c_{\alpha}(t)^{-1} \\
& =q\left(w^{L}\right) \prod_{\alpha \in R_{1,+} \backslash R_{L, 1,+}} \frac{|1-\alpha(t)|^{2}}{\left|1+q_{\alpha^{\vee}}^{1 / 2} \alpha(t)^{1 / 2}\right|^{2}\left|1-q_{\alpha^{\vee}}^{1 / 2} q_{2 \alpha^{\vee}} \alpha(t)^{1 / 2}\right|^{2}}
\end{aligned}
$$

Then $m^{L}$ and $m_{L}$ are Aut $\left(W_{0}\right)$-equivariant, i.e. $m^{L}(t)=$ $m^{g L}(g t)$ and $m_{L}(t)=m_{g L}(g t)$ for every $g \in \operatorname{Aut}\left(W_{0}\right)$. In particular, $m^{L}$ and $m_{L}$ are invariant for the stabilizer $N_{L}$ of $L$ in $W_{0}$.

(iii) We have $\kappa_{L}=k_{L} \kappa_{\mathcal{R}_{L},\left\{r_{L}\right\}}, \bar{\kappa}_{W_{L} L}=k_{L} \bar{\kappa}_{\mathcal{R}_{L}, W_{L} r_{L}}$.

(iv) For $z \in \mathcal{Z}$, we have

$$
\frac{1}{\left|W_{0} L\right|} \int_{T} z d \nu_{W_{0} L}=k_{L} \nu_{\mathcal{R}_{L},\left\{r_{L}\right\}}\left(\left\{r_{L}\right\}\right) \int_{L^{\text {temp }}} z(t) m^{L}(t) d^{L}(t) .
$$

(v) Assuming that $q$ is expressed as in Convention 2.1 with $f_{s} \in$ $2 \mathbb{Z}$. Then $\nu_{\mathcal{R}_{L},\left\{r_{L}\right\}}\left(\left\{r_{L}\right\}\right)=\bar{\kappa}_{\mathcal{R}_{L}, W_{L} r_{L}} m_{\mathcal{R}_{L},\left\{r_{L}\right\}}\left(r_{L}\right)$ is of the form $d \mathbf{q}^{n} f(\mathbf{q})$, where $d \in \mathbb{Q}, n \in \mathbb{Z}$, and where $f$ is a quotient of products of cyclotomic polynomials in $\mathbf{q}$.

Proof. (i). We note that for $w \in W^{L}, t_{0}$ and $w^{-1} t_{0}$ are in the same chamber of $\mathcal{L}^{L}$. Hence, by application of Proposition 3.10, we may replace $\xi_{w L}$ by $w\left(\xi_{L}\right)$.

(ii). This is trivial.

(iii). The formula $\kappa_{L}=k_{L} \kappa_{\mathcal{R}_{L},\left\{r_{L}\right\}}$ was explained in the proof of Theorem 3.25. Let $W_{L}=W\left(R_{L}\right)$. Let $N_{T^{L}}$ be the stabilizer of $T^{L}$ in $W_{0}$. Observe that $N_{L} \subset N_{T^{L}}$ and $W_{L} \triangleleft N_{T^{L}}$. If we define $\Gamma_{L}=$ $N_{T^{L}} \cap W^{L}$ then $\Gamma_{L}$ is a complementary subgroup of $W_{L}$ in $N_{T^{L}}$. Using (i), (ii) and the remark $\kappa_{L}=k_{L} \kappa_{\mathcal{R}_{L},\left\{r_{L}\right\}}$ we see that

$$
\begin{aligned}
\bar{\kappa}_{W_{L} L} & =\frac{1}{\left|W_{0} L\right|} \sum_{L^{\prime} \in W_{0} L} \kappa_{L^{\prime}} \\
& =\frac{\left|W_{0} T_{L}\right|}{\left|W_{0} L\right|} \sum_{L^{\prime} \in N_{T^{L}} L} \kappa_{L^{\prime}} \\
& =\frac{\left|W_{0} T_{L}\right|\left|N_{T^{L}} L\right|}{\left|W_{0} L\right|} k_{L} \bar{\kappa}_{\mathcal{R}_{L}, W_{L} r_{L}}=k_{L} \bar{\kappa}_{\mathcal{R}_{L}, W_{L} r_{L}}
\end{aligned}
$$

Using Theorem 3.25 and equation (3.53) the result follows.

(iv). Follows easily from (iii). 
(v). Since equation (3.47) involves only roots in $R_{0}$, it is sufficient to prove the statement for $R_{0}$ indecomposable and $X=Q$. Notice that for all $\alpha \in R_{0}, \alpha(s)$ is a root of unity and, by Theorem 7.14(iii), $\alpha(c)$ is an integral power of $\mathbf{q}$. Looking at the explicit formula (3.47), we see that it remains to show that this expression has rational coefficients if $L=r=s c$ is a residual point. Let $k$ be the extension of $\mathbb{Q}$ by the values of $\alpha(s)$, where $\alpha$ runs over $R_{0}$. In the case where $\mathcal{R}$ is of type $C_{n}^{\text {aff }}$ it follows by Lemma 7.6 that $k=\mathbb{Q}$, and we are done. For the other classical cases it follows from the result of Borel and de Siebenthal [8] that the order of $s$ is at most two, and hence that $k=\mathbb{Q}$. Next let $\mathcal{R}$ be of exceptional type, and $\sigma \in \operatorname{Gal}(k / \mathbb{Q})$. Define a character $\sigma(s)$ of $X=$ $Q$ by $Q \ni x \rightarrow \sigma(x(s))=: x(\sigma(s))$. By Lemma 7.8 we see that there exists a $w_{1} \in W_{0}$ such that $\sigma(s)=w_{1} s$. Moreover, $w_{1}: R_{s, 0} \rightarrow R_{s, 0}$ acts as an automorphism and $c$ is an $R_{s, 0}$-residual point. If $F_{s, 0}$ contains isomorphic components then these are of type $A$, which has only one real residual point up to the action of $W\left(R_{s, 0}\right)$. Hence by Theorem 7.14(i), there exists a $w_{2} \in W\left(R_{s, 0}\right)$ such that $w_{1}(c)=w_{2}(c)$. Put $w=w_{2}^{-1} w_{1}$, so that $w r=c \sigma(s)$. By the $W_{0}$-equivariance of $m_{\{r\}}(r)$ we see that (with the action of $\sigma$ being extended to $k\left[\mathbf{q}, \mathbf{q}^{-1}\right]$ by its action on the coefficients) $\sigma\left(m_{\{r\}}(r)\right)=m_{\{w r\}}(w r)=m_{\{r\}}(r)$, whence the desired rationality.

The next proposition is a direct consequence of (the proof of) Theorem 3.25 and the definition of $\chi_{t}$.

Proposition 3.28. Let $r=s c \in T$ be a residual point, and let $a \in \mathcal{A}$. Then

$$
\nu\left(W_{0} r\right) \chi_{r}(a)=m_{\{r\}}(r) \sum_{r^{\prime} \in W_{0} r} \kappa_{\left\{r^{\prime}\right\}} a\left(r^{\prime}\right) .
$$

Theorem 3.29. The support of $\nu$ is exactly equal to the union of the tempered residual cosets. In other words, $S=W_{0} \backslash \cup_{L}$ residual $L^{\text {temp }}$.

Proof. The equality $S=W_{0} \backslash \operatorname{Supp}(\nu)$ was explained in 2.9.1, so it suffices to show that the support of $\nu$ is equal to the union of the tempered residual cosets. By Theorem 3.25 we know that $\nu$ is supported on this set, so we need only to show that $W_{0} L^{t e m p}$ is contained in the support for each tempered residual coset $L$.

By Proposition 3.27 this reduces to the case of a residual point $r=s c$. By Proposition 3.28 it is enough to show that there exists at least one $r^{\prime}=w r \in W_{0} r$ such that $\kappa_{\left\{r^{\prime}\right\}} \neq 0$. In other words, using Proposition 3.28 we single out the point residue at $r^{\prime}$. In particular, we ignore all residues at residual cosets which do not contain $r^{\prime}$ and thus do not contribute to $\kappa_{\left\{r^{\prime}\right\}}$ in the argument below. 
By the $W_{0}$-invariance of $\omega$, we can formulate the problem as follows. Recall from the proof of Theorem 3.25 that

$$
\kappa_{\{r\}} m_{\{r\}}(r)=\int_{\xi} \omega
$$

where $\xi$ is the residue cycle at $r$, which is obtained from Proposition 3.7 if we use the $n$-form

$$
\omega(t)=\frac{d t}{c(t) c\left(t^{-1}\right)}
$$

and a base point $t_{0} \in T_{r s}$ such that $\forall \alpha_{i} \in F_{0}: \alpha_{i}\left(t_{0}\right)<q\left(s_{i}\right)$. By definition, $m_{\{r\}}(r) \neq 0$. For $r^{\prime}=w r$ we have

$$
\kappa_{\left\{r^{\prime}\right\}} m_{\{r\}}(r)=\int_{\xi(w)} \omega,
$$

where $\xi(w)$ is the cycle near $r$ which we obtain in Proposition 3.7 when we replace $t_{0}$ by $w^{-1} t_{0}$. Hence we have to show that there exists a proper choice for $t_{0}$ such that when we start the contour shift algorithm from this point, the corresponding point residue at $r$ will be nonzero. The problem we have to surmount is possible cancellation of nonzero contributions to $\kappa_{\left\{r^{\prime}\right\}}$. We will do this by showing that there exists at least one chamber such that the residue at $r$ consists only of one nonzero contribution.

We consider the real arrangement $\mathcal{L}^{\{r\}}$ in $T_{r s}$, and transport the Euclidean structure of $\mathfrak{t}$ to $T_{r s}$ by means of $t \rightarrow \log \left(c^{-1} t\right)$. Then $\mathcal{L}^{\{r\}}$ is the lattice of intersections of a central arrangement of hyperplanes with center $c$. We assign indices $i_{L}$ to the elements of $\mathcal{L}^{\{r\}}$ by considering the index of the corresponding complex coset containing $r$, and we note that by Corollary 7.12, $i_{\{r\}}=n:=\operatorname{codim}(\{r\})$. From Corollary 7.12 we further obtain the result that there exist full flags of subspaces $c_{L} T_{r s}^{L} \in \mathcal{L}^{\{r\}}$ such that $i_{L}=\operatorname{codim}(L)$. In particular, there exists at least one line $l$ through $r$ with $i_{l}=n-1$.

By Theorem 7.17 we see that the centers $c_{L}, c_{L^{\prime}}$ of two "residual subspaces" $c_{L} T^{L} \subset c_{L^{\prime}} T^{L^{\prime}}$ (i.e. $\operatorname{codim}\left(T^{L}\right)=i_{L}$ and $\left.\operatorname{codim}\left(T^{L^{\prime}}\right)=i_{L^{\prime}}\right)$ in $\mathcal{L}^{\{r\}}$ satisfy $c_{L^{\prime}} \neq c_{L}$ unless $c_{L} T^{L}=c_{L^{\prime}} T^{L^{\prime}}$. Hence $d\left(e, c_{L^{\prime}}\right) \leq d\left(e, c_{L}\right)$ (where $d$ denotes the distance function), with equality only if $c_{L} T^{L}=$ $c_{L^{\prime}} T^{L^{\prime}}$. In the case of a residual line $l \in \mathcal{L}^{\{r\}}, c T^{l}$ is divided in two half lines by $c$, and $c_{l}$ lies in one of the two halves (i.e. does not coincide with $c$ ).

We want to find a chamber for $t_{0}$ such that the corresponding point residue $\kappa_{\left\{r^{\prime}\right\}} m_{\{r\}}(r)$ at $r$ is nonzero. We argue by induction on the rank. If the rank of $R_{0}$ is 1 , obviously we get $\kappa_{\left\{r^{\prime}\right\}} \neq 0$ if we choose $t_{0}$ 
in the half line not containing $e=c_{T}$, because we then have to pass a simple pole of $\omega$ at $r$ when moving the contour $t_{0} T_{u}$ to $T_{u}$ (since $t_{0}$ and $e=c_{T}$ are separated by $c$ ). Assume by induction that for any residual point $p$ of a rank $n-1$ root system, we can choose a chamber for $t_{0}$ such that $\kappa_{\{p\}} \neq 0$. Let $S \subset T_{r s}$ be a sphere centered at $r$ through $e$, and consider the configuration of hyperspheres in $\mathcal{L}^{\{r\}} \cap S$. Let us call $e \in S$ the north pole of $S$. If $L_{S}=c_{L} T_{r s}^{L} \cap S$ with $\operatorname{dim}\left(T^{L}\right)>1$, we denote by $c_{L \cap S}$ the intersection of the half line through $c_{L}$ beginning in $c$ (recall that $c \neq c_{L}$ ) and $L_{S}$. By the above remarks, $c_{L \cap S}$ is in the northern hemisphere for all residual $L \supset r$ of dimension $>1$. We call this point $c_{L \cap S}$ the center of $L_{S}$.

In the case when $\operatorname{dim}\left(T^{L}\right)=1, L_{S}$ is disconnected and consists of two antipodal points $c_{L \cap S}$ (north) and $\overline{c_{L \cap S}}$ (south), its opposite. In this case of residual lines through $r$, both of these antipodal points are considered as centers of $\mathcal{L}^{\{r\}} \cap S$. We call $c_{L \cap S}$ the northern center, and its opposite is called the southern center. All centers of $\mathcal{L}^{\{r\}} \cap S$ lie in the northern hemisphere, with the exception of the southern centers of the residual lines through $r$.

Consider a closed (spherical) ball $D \subset S$ centered at $e$ such that $D$ contains a southern center $p$ in its boundary, but no southern centers in its interior. Since $e$ is regular with respect to $\mathcal{L}^{\{r\}}$ (a trivial case of Theorem 7.17, as $e$ is the center of $T$ ), we have $D \neq S$.

We take $t_{0}$ in $S$, and we apply the algorithm as described in the proof of Proposition 3.7, but now on the sphere $S$, and with respect to the sets $L_{S}$ and their centers.

By the induction hypothesis, we can take $t_{0} \in S$ close to $p$ in a chamber of the configuration $\mathcal{L}^{\{r\}} \cap S$ which contains $p$ in its closure, such that a nonzero residue at $l$ is picked up in $p$. Denote by $\mathcal{L}^{p} \cap S$ the central subarrangement of elements of $\mathcal{L}^{\{r\}} \cap S$ containing $p$. Consider any alternative "identity element" $\tilde{e}$ which belongs to the same chamber of the dual configuration of $\mathcal{L}^{p} \cap S$ as the real (original) identity element $e$.

As was explained in (the proof of) Proposition 3.13, when we apply the contour shifts as in (the proof of) Proposition 3.7 to $\mathcal{L}^{\{r\}} \cap S$, the residue at $p$ only depends on the dual chamber which contains the identity element. In other words, we may use the new identity $\tilde{e}$ instead of $e$ without changing the residue at $p$. We can and will choose $\tilde{e}$ close to $p$, and in the interior of $D$. By Proposition 3.7 we can replace the integral over $t_{0} T_{u}$ by a sum of integrals over cosets of the form $\tilde{c}_{L \cap S} \tilde{s}_{L} T_{u}^{L}$ (for some $\tilde{s}_{L} \in T_{u}$ ) of the residue kernel $\tilde{\kappa}_{L} m_{L}$ (cf. equation (3.51)) on $L$. As was mentioned above, we are only interested in such 
ON THE SPECTRAL DECOMPOSITION OF AFFINE HECKE ALGEBRAS 55

contributions when $r \in L$, which means that we may take $\tilde{s}_{L}=s$. The new "centers" $\tilde{c}_{L \cap S}$ with respect to the new identity element $\tilde{e}$ are in the interior of $D$.

Next we apply the algorithm of contour shifts as in Proposition 3.7 to move the cycles $\tilde{c}_{L \cap S} s T_{u}^{L}$ to $c_{L \cap S} s T_{u}^{L}$. Since both the new centers $\tilde{c}_{L \cap S}$ and the original centers $c_{L \cap S}$ belong to the interior of $D$, and since the intersection of $D$ with $L_{S}$ is connected if $\operatorname{dim}\left(L_{S}\right)>0$, we can choose every path in the contour shifting algorithm inside the interior of $D$. Thus, the centers $c_{L \cap S}$ of the residual cosets $L$ that arise in addition the one southern center $c_{l}=p$ in the above process are in the interior of $D$. In particular, with the exception of $p s T_{u}^{l}$, the one dimensional cosets of integration which show up in this way, all have a northern center.

Finally, in order to compute the residue $\kappa_{r^{\prime}} m_{\{r\}}(r)$ at $r^{\prime}$, we now have to move the center $c_{L \cap S} \in S$ of $L_{S}$ to the corresponding center $c_{L} \in T_{r s}$ of $L$, for each residual coset $L$ which contains $r$ and which

contributes to $\int_{t_{0} T_{u}} \omega$. The only such center of $\mathcal{L}^{\{r\}} \cap S$ which will cross $c$ is the southern center $p$. Since $m_{l}$ has a simple pole at $r=s c$, we conclude that this gives a nonzero residue at $r$. Hence with the above choice of $t_{0}$ we get $\kappa_{\left\{r^{\prime}\right\}} \neq 0$, which is what we wanted to show.

\subsection{Discrete series}

In this subsection we show that the irreducible characters $\chi_{r, i}$ (see Definition 3.24) associated to a residual point are in fact discrete series characters.

Corollary 3.30. (of Theorem 3.29) For every residual point $r=s c$, the sum $\bar{\kappa}_{W_{0} r}\left|W_{0} r\right|=\sum_{r^{\prime} \in W_{0} r} \kappa_{r^{\prime}} \neq 0$, and for all $a \in \mathcal{A}$ :

$$
\chi_{r}(a)=\frac{1}{\bar{\kappa}_{W_{0} r}\left|W_{0} r\right|} \sum_{r^{\prime} \in W_{0} r} \kappa_{\left\{r^{\prime}\right\}} a\left(r^{\prime}\right) .
$$

Moreover, $\kappa_{\left\{r^{\prime}\right\}}=0$ unless $\forall x \in X^{+} \backslash\{0\}:\left|x\left(r^{\prime}\right)\right|<1$ (where $X^{+}$ denotes the set of dominant elements in $X$ ).

Proof. This is immediate from Proposition 3.28 and Theorem 3.29, except for the last assertion. This fact follows from Proposition 3.12. We know that $e$ is regular in $\mathcal{L}_{\left\{r^{\prime}\right\}}$ by Theorem 7.17. On the other hand, $t_{0}$ lies in $c^{\prime} T_{r s,-}$, which is clearly a subset of a chamber of $\mathcal{L}^{\left\{r^{\prime}\right\}}$. The anti-dual of the chamber of $\mathcal{L}^{\left\{r^{\prime}\right\}}$ containing $t_{0}$ is thus a subset of $c^{\prime} T_{r s}^{+}$, with $T_{r s}^{+}:=\left\{t \in T_{r s} \mid \forall x \in X^{+} \backslash\{0\}: x(t)>1\right\}$. Thus when $e$ is contained in the anti-dual chamber we have $c^{\prime} \in T_{r s}^{-}$as desired. 
We introduce the notation $\Delta_{\mathcal{R}}\left(=\Delta_{\mathcal{R}, q}\right)$ for a complete set of representatives of the finite set of equivalence classes of the irreducible discrete series representations of $\mathcal{H}(\mathcal{R}, q)$, and $\Delta_{\mathcal{R}, W_{0} r}\left(=\Delta_{\mathcal{R}, W_{0} r, q}\right)$ for the representatives of the classes of irreducible discrete series of $\mathcal{H}(\mathcal{R}, q)$ with central character $W_{0} r$. (We sometimes drop $\mathcal{R}$ from the notation if no confusion is possible, and write $\Delta_{W_{0} r}$.)

Lemma 3.31. $\Delta_{W_{0} r}$ is nonempty if and only if $r$ is residual. If $r$ is residual, $\Delta_{W_{0} r}$ is in bijective correspondence with the collection $\left\{\delta_{r, i}\right\}$ of irreducible characters of $\overline{\mathcal{H}^{r}}$. In particular, $\mathcal{H}(\mathcal{R}, q)$ has at most finitely many discrete series representation.

Proof. We have

$$
\chi_{r, i}(a)=\sum_{r^{\prime} \in W_{0} r} \operatorname{dim}\left(V_{r, i}^{r^{\prime}}\right) a\left(r^{\prime}\right) .
$$

Hence from $d_{r, i}>0$,

$$
\chi_{r}(a)=\sum_{i} \chi_{r, i}(a) d_{r, i}
$$

and Corollary 3.30 we conclude that the generalized weight spaces of $V_{r, i}$ indeed satisfy the Casselman criterion Lemma 2.22 for discrete series.

Conversely, if $\delta$ is a discrete series representation, Theorem $2.25 \mathrm{im}$ plies that $\mu_{P l}(\delta)>0$. By Corollary 3.23, the central character $W_{0} r$ of $\delta$ is such that $\nu(\{r\})>0$. Theorem 3.25 implies that such points $r$ are necessarily residual.

In view of the above, we adapt the notations of Definition 3.24 accordingly, i.e. we write $d_{\mathcal{R}, \delta}$ (or simply $d_{\delta}$ ) instead of $d_{r, i}$ if $\delta \in \Delta_{\mathcal{R}, W_{0} r}$, and its character $\chi_{\delta}$ descends to $\chi_{r, i}$ on $\overline{\mathcal{H}^{r}}$ etc.

Corollary 3.32. Let $\delta \in \Delta_{W_{0} r}$. The formal dimension $\mu_{P l}(\delta)$ of $\delta$ equals

$$
\mu_{P l}(\delta)=\operatorname{fdim}(\delta)=d_{\delta} \nu\left(\left\{W_{0} r\right\}\right)=\left|W_{0} r\right| \bar{\kappa}_{W_{0} r} d_{\delta} m_{\{r\}}(r)
$$

Proof. Combine equation (2.31), Corollary 3.23, and Theorem 3.25.

Corollary 3.33. For a residual point $r$ there exist constants $C, \epsilon>0$ such that

$$
\left|\chi_{r}\left(N_{w}\right)\right| \leq C \exp (-\epsilon l(w)) .
$$


Corollary 3.34. The residual degrees $d_{\delta}>0$ of the irreducible characters $\chi_{\delta}$ of the residual algebra $\overline{\mathcal{H}^{r}}$ (with $r$ a residual point) satisfy the following system of linear equations.

$$
\sum_{\delta \in\left[\Delta_{\mathcal{R}, W_{0} r}\right]} \operatorname{dim}\left(V_{\delta}^{r^{\prime}}\right) d_{\delta}=\frac{\kappa_{\left\{r^{\prime}\right\}}}{\bar{\kappa}_{W_{0} r}\left|W_{0} r\right|} .
$$

(with $V_{\delta}^{r^{\prime}}$ the generalized $r^{\prime}$-weight space in the space $V_{\delta}$ of $\delta$ ). In particular we conclude that the nonzero $\kappa_{\left\{r^{\prime}\right\}}$ all have the same sign (equal to the sign of $\left.m_{\{r\}}(r)\right)$.

Remark 3.35. We note in addition that if the restrictions $\left.\chi_{\delta}\right|_{\mathcal{A}}$ to $\mathcal{A}$ of the characters $\chi_{\delta}$ are linearly independent, it follows from the equations (3.70) that $d_{\delta} \in \mathbb{Q}$ for all $\delta \in\left[\Delta_{\mathcal{R}}\right]$. I did not find any argument in favor of this linear independence. However, we do conjecture that the constants $d_{\delta}$ are rational, see Conjecture 2.2\%.

\subsection{Temperedness of the traces $\chi_{t}$}

In this subsection we discuss the tempered growth behaviour of the $\chi_{t}$ on the orthonormal basis $N_{w}$ of $\mathcal{H}$, as a corollary of the analysis of the $\mathcal{A}$-weights of $\chi_{t}$.

Proposition 3.36. Let $L$ be residual such that $W_{L}$ is a standard parabolic subgroup of $W_{0}$. For $t \in L^{\text {temp }}$ we write $t=r_{L} t^{L}$, with $t^{L} \in T_{u}^{L}$. We consider $\left.\chi_{t}\right|_{\mathcal{A}}$ as a formal linear combination of elements of $T$. Likewise, let $\mathcal{A}_{L}=\mathbb{C}\left[X_{L}\right]$ be the ring of regular functions on $T_{L} \subset T$. We consider $\left.\chi_{\mathcal{R}_{L},\left\{r_{L}\right\}}\right|_{\mathcal{A}_{L}}$ as a formal linear combination of elements of $T_{L}$. In this sense we have, $\nu_{L}$-almost everywhere on $L^{\text {temp }}$,

$$
\left.\chi_{t}\right|_{\mathcal{A}}=\frac{1}{\left|W^{L}\right|} \sum_{w \in W^{L}} w\left(\left.t^{L} \chi_{\mathcal{R}_{L},\left\{r_{L}\right\}}\right|_{\mathcal{A}_{L}}\right) .
$$

Hence $\nu$-almost everywhere, $\chi_{t}$ is a nonzero tempered functional on $\mathcal{H}$.

Proof. Equation (3.71) follows by a straightforward computation similar to Proposition 3.28, using Proposition 3.27 and the definition of $\chi_{t}$. Since $\chi_{t}$ is a positive combination of the irreducible characters of the residual algebra $\overline{\mathcal{H}^{t}}$, it follows that the weights $t^{\prime} \in W_{0} t$ of the generalized $\mathcal{A}$-eigenspaces of the irreducible characters of $\overline{\mathcal{H}^{t}}$ all satisfy the condition $\forall x \in X^{+}:\left|x\left(t^{\prime}\right)\right| \leq 1$. This shows, by Casselman's criterion Lemma 2.20, that $\chi_{t}$ is a tempered functional on $\mathcal{H}$. 


\section{Localization of the Hecke algebra}

We have obtained thus far a decomposition of the trace $\tau$ as an integral of positive, finite traces $\chi_{t}$ against an explicit probability measure $\nu$ on $T$, such that each $\chi_{t}$ is a finite positive linear combination of finite dimensional, irreducible characters of $\mathfrak{C}$. This is an important step towards our goal of finding the Plancherel decomposition, but it is not yet satisfactory because we know virtually nothing about the behavior of the decomposition of $\chi_{t}$ in irreducible characters at this stage, neither as a function of $t$, nor as a function of $\mathbf{q}$. In particular, the residual degrees $d_{t, i} \in \mathbb{R}_{+}$of the residual algebras are obscure at this point, and these degrees are involved in the Plancherel measure $\mu_{P l}$.

In the remaining part of the paper we will formulate the Plancherel theorem, and also remedy to some extend the above problems. The support $S$ of $\nu$ (viewed as a $W_{0}$-invariant measure on $T$ ) decomposes as a union of the closed sets $L^{\text {temp }}$ (see 2.9.1). For each $L$ we show that, up to isomorphism of Hilbert algebras, the residual algebras $\overline{\mathcal{H}^{t}}$ are independent of $t, \nu$-almost everywhere on $L^{t e m p}$.

The above is based on ideas of Lusztig [26] about completions of the affine Hecke algebra. Lusztig describes the $\mathcal{I}_{t}$-adic completion of $\mathcal{H}$, where $\mathcal{I}_{t}$ is a maximal ideal of $\mathcal{Z}$. It is not hard to see that Lusztig's arguments can be adapted to (analytic) localization with respect to suitably small open neighborhoods $U \supset W_{0} t$ of orbits of points in $T$, and this will be discussed in present section.

When $s=s_{\alpha} \in S_{0}$ (with $\alpha \in F_{1}$ ), we define an intertwining element $\iota_{s}$ as follows:

$$
\begin{aligned}
\iota_{s} & =\left(1-\theta_{-\alpha}\right) T_{s}+\left(\left(1-q_{\alpha \vee} q_{2 \alpha^{\vee}}\right)+q_{\alpha^{\vee}}^{1 / 2}\left(1-q_{2 \alpha^{\vee}}\right) \theta_{-\alpha / 2}\right) \\
& =T_{s}\left(1-\theta_{\alpha}\right)+\left(\left(q_{\alpha \vee} q_{2 \alpha^{\vee}}-1\right) \theta_{\alpha}+q_{\alpha^{\vee}}^{1 / 2}\left(q_{2 \alpha^{\vee}}-1\right) \theta_{\alpha / 2}\right)
\end{aligned}
$$

We remind the reader of the convention of Remark 3.1. These elements are important tools to study the Hecke algebra. We recall from [37], Theorem 2.8 that these elements satisfy the braid relations, and they satisfy (for all $x \in X$ )

$$
\iota_{s} \theta_{x}=\theta_{s(x)} \iota_{s}
$$

and finally they satisfy

$$
\iota_{s}^{2}=\left(q_{\alpha^{\vee}}^{1 / 2}+\theta_{-\alpha / 2}\right)\left(q_{\alpha^{\vee}}^{1 / 2}+\theta_{\alpha / 2}\right)\left(q_{\alpha^{\vee}}^{1 / 2} q_{2 \alpha^{\vee}}-\theta_{-\alpha / 2}\right)\left(q_{\alpha^{\vee}}^{1 / 2} q_{2 \alpha^{\vee}}-\theta_{\alpha / 2}\right) .
$$

(where we have again used the convention of Remark 3.1!). Suitably normalized versions of the $\iota_{s}$ generate a group isomorphic to the Weyl group $W_{0}$. In order to normalize the intertwiners, we need to tensor $\mathcal{H}$ by the field of fractions $\mathcal{F}$ of the center $\mathcal{Z}$. So let us introduce the 
ON THE SPECTRAL DECOMPOSITION OF AFFINE HECKE ALGEBRAS 59

algebra

$$
{ }_{\mathcal{F}} \mathcal{H}:=\mathcal{F} \otimes_{\mathcal{Z}} \mathcal{H}
$$

with the multiplication defined by $(f \otimes h)\left(f^{\prime} \otimes h^{\prime}\right):=f f^{\prime} \otimes h h^{\prime}$. Notice that this an algebra over $\mathcal{F}$ of dimension $\left|W_{0}\right|^{2}$. The subalgebra ${ }_{\mathcal{F}} \mathcal{A}=\mathcal{F} \otimes_{\mathcal{Z}} \mathcal{A}$ is isomorphic to the field of fractions of $\mathcal{A}$. The field extension $\mathcal{F} \subset{ }_{\mathcal{F}} \mathcal{A}$ has Galois group $W_{0}$, and we denote by $f \rightarrow f^{w}$ the natural action of $W_{0}$ on the field of rational functions on $T$. The elements $T_{w}$ with $w \in W_{0}$ form a basis for ${ }_{\mathcal{F}} \mathcal{H}$ for multiplication on the left or multiplication on the right by ${ }_{\mathcal{F}} \mathcal{A}$, in the sense that

$$
{ }_{\mathcal{F}} \mathcal{H}=\oplus_{w \in W_{0}} \mathcal{F} \mathcal{A} T_{w}=\oplus_{w \in W_{0}} T_{w \mathcal{F}} \mathcal{A} .
$$

The algebra structure of ${ }_{\mathcal{F}} \mathcal{H}$ is determined by the Bernstein-ZelevinskiLusztig relations as before: when $f \in{ }_{\mathcal{F}} \mathcal{A}$ and $s=s_{\alpha}$ with $\alpha \in F_{1}$, we have

$$
f T_{s}-T_{s} f^{s}=\left(\left(q_{2 \alpha} \vee q_{\alpha} \vee-1\right)+q_{\alpha^{\vee}}^{1 / 2}\left(q_{2 \alpha^{\vee}}-1\right) \theta_{-\alpha / 2}\right) \frac{f-f^{s}}{1-\theta_{-\alpha}}
$$

We have identified $\mathcal{A}$ with the algebra of regular functions on $T$ in the above formula.

Let us introduce

$$
\begin{aligned}
n_{\alpha} & :=q\left(s_{\alpha}\right) \Delta_{\alpha} c_{\alpha} \\
& =\left(q_{\alpha^{\vee}}^{1 / 2}+\theta_{-\alpha / 2}\right)\left(q_{\alpha \vee}^{1 / 2} q_{2 \alpha^{\vee}}-\theta_{-\alpha / 2}\right) \in \mathcal{A},
\end{aligned}
$$

where we used the Macdonald $c$-function introduced in equation (3.3) and (3.4).

The normalized intertwiners are now defined by (with $s=s_{\alpha}, \alpha \in$ $\left.R_{1}\right)$ :

$$
\iota_{s}^{0}:=n_{\alpha}^{-1} \iota_{s} \in{ }_{\mathcal{F}} \mathcal{H} .
$$

By the properties of the intertwiners listed above it is clear that $\left(\iota_{s}^{0}\right)^{2}=$ 1. In particular, $\iota_{s}^{0} \in \mathcal{F}_{\mathcal{H}} \mathcal{H}^{\times}$, the group of invertible elements of ${ }_{\mathcal{F}} \mathcal{H}$. From the above we have the following result:

Lemma 4.1. The map $S_{0} \ni s \rightarrow \iota_{s}^{0} \in{ }_{\mathcal{F}} \mathcal{H}^{\times}$extends (uniquely) to a homomorphism $W_{0} \ni w \rightarrow \iota_{w}^{0} \in \mathcal{F}_{\mathcal{H}} \mathcal{H}^{\times}$. Moreover, for all $f \in{ }_{\mathcal{F}} \mathcal{A}$ we have that $\iota_{w}^{0} f \iota_{w^{-1}}^{0}=f^{w}$.

Lusztig ([26], Proposition 5.5) proved that in fact

\section{Theorem 4.2.}

$$
{ }_{\mathcal{F}} \mathcal{H}=\oplus_{w \in W_{0}} \iota_{w}^{0} \mathcal{F} \mathcal{A}=\oplus_{w \in W_{0}} \mathcal{F} \mathcal{A} \iota_{w}^{0}
$$


Let $U \subset T$ be a nonempty, open, $W_{0}$-invariant subset. We denote by $\mathcal{Z}^{a n}(U)$ the ring of $W_{0}$-invariant holomorphic functions of $U$. Consider the algebras $\mathcal{A}^{a n}(U):=\mathcal{Z}^{a n}(U) \otimes_{\mathcal{Z}} \mathcal{A}$ and $\mathcal{H}^{a n}(T):=\mathcal{Z}^{a n}(T) \otimes_{\mathcal{Z}} \mathcal{H}$. The algebra structure on $\mathcal{H}^{a n}(T)$ is defined by $(f \otimes h)\left(f^{\prime} \otimes h^{\prime}\right):=f f^{\prime} \otimes h h^{\prime}$ (similar to the definition of ${ }_{\mathcal{F}} \mathcal{H}$ ).

Let us first remark that the finite dimensional representation theory of the "analytic" affine Hecke algebra $\mathcal{H}^{a n}(T)$ is the same as the finite dimensional representation theory of $\mathcal{H}$. Every finite dimensional representation $\pi$ of $\mathcal{H}$ determines a co-finite ideal $J_{\pi} \subset \mathcal{Z}$, the ideal of central elements of $\mathcal{H}$ which are annihilated by $\pi$. Denote by $J_{\pi}^{a n}$ the ideal of $\mathcal{Z}^{a n}(T)$ generated by $J_{\pi}$. Because of the co-finiteness we have an isomorphism

$$
\mathcal{Z} / J_{\pi} \stackrel{\sim}{\rightarrow} \mathcal{Z}^{a n}(T) / J_{\pi}^{a n}(T) .
$$

This shows that $\pi$ can be uniquely lifted to a representation $\pi^{a n}$ of $\mathcal{H}^{a n}(T)$ whose restriction to $\mathcal{H}$ is $\pi$. The functor $\pi \rightarrow \pi^{a n}$ defines an equivalence between the categories of finite dimensional representations of $\mathcal{H}$ and $\mathcal{H}^{a n}(T)$ (with the inverse given by restriction).

For any $W_{0}$-invariant nonempty open set $U \subset T$ we define the localized affine Hecke algebra

$$
\mathcal{H}^{a n}(U):=\mathcal{Z}^{a n}(U) \otimes_{\mathcal{Z}} \mathcal{H}
$$

This defines a presheaf of $\mathcal{Z}^{a n}$-algebras on $W_{0} \backslash T$, which is finitely generated over the analytic structure sheaf $\mathcal{Z}^{\text {an }}$ of the geometric quotient $W_{0} \backslash T$.

A similar argument as above shows that

Proposition 4.3. The category $\operatorname{Rep}\left(\mathcal{H}^{a n}(U)\right)$ of finite dimensional modules $\pi_{U}^{a n}$ over $\mathcal{H}^{a n}(U)$ is equivalent to the category $\operatorname{Rep}_{U}(\mathcal{H})$ of finite dimensional modules $\pi$ over $\mathcal{H}$ whose $\mathcal{Z}$-spectrum is contained in $U$.

Lemma 4.4. For every $W_{0}$-invariant nonempty open set $U$ in $T$, we have the isomorphism $\mathcal{A}^{\text {an }}(U) \simeq \mathcal{Z}^{\text {an }}(U) \otimes_{\mathcal{Z}} \mathcal{A}$, where $\mathcal{A}^{\text {an }}(U)$ denotes the ring of analytic functions on $U$.

Proof. Both the left and the right hand side are finitely generated modules over $\mathcal{Z}^{\text {an }}(U)$, and we have a natural morphism from the right hand side to the left hand side (product map). In order to prove that this map is an isomorphism it suffices to show this in the stalks of the corresponding sheaves at each point of $W_{0} \backslash U$. Let $\mathcal{I}_{t}$ denote the maximal ideal in $\mathcal{Z}$ corresponding to $W_{0} t$, and let $\hat{\mathcal{Z}}_{t}$ denote the $\mathcal{I}_{t}$-adic com-

pletion. Because $\hat{\mathcal{Z}}_{t}$ is faithfully flat over $\mathcal{Z}_{t}^{\text {an }}$ (the stalk at $W_{0} t$ of the 
ON THE SPECTRAL DECOMPOSITION OF AFFINE HECKE ALGEBRAS 61 sheaf $\left.\mathcal{Z}^{a n}\right)$, it suffices to check that for each $t \in U$, we have

$$
\hat{\mathcal{Z}}_{t} \otimes_{\mathcal{Z}_{t}^{a n}} \mathcal{A}_{W_{0} t}^{a n} \simeq \hat{\mathcal{Z}}_{t} \otimes_{\mathcal{Z}} \mathcal{A}
$$

where $\mathcal{A}_{W_{0} t}^{a n}=\oplus_{t^{\prime}} \mathcal{A}_{t^{\prime}}^{a n}$ denotes the space of analytic germs at the set $W_{0} t$. Let $m_{t}$ denote the maximal ideal of $\mathcal{A}$ at $t \in T$, and let $\mathcal{I}_{t} \mathcal{A}=$ $\prod_{t^{\prime} \in W_{0} t} j_{t^{\prime}}$ with $j_{t^{\prime}}=\mathcal{I}_{t} \mathcal{A} \cap m_{t^{\prime}}$. For all $t^{\prime} \in W_{0} t$ we have $\widehat{\mathcal{A}_{t^{\prime}}^{a n}} j_{t^{\prime}} \mathcal{A}_{t^{\prime}}^{a n}=$ $\hat{\mathcal{A}}_{j_{t^{\prime}}}$. Since $A_{t}^{a n} \cap \mathcal{I}_{t} A_{W_{0} t}^{a n}=j_{t} \mathcal{A}_{t}^{a n}$, the left hand side of 4.10 is equal to $\oplus_{t^{\prime} \in W_{0} t} \hat{\mathcal{A}}_{j_{t^{\prime}}}$, the sum of the completions of $\mathcal{A}$ with respect to $j_{t^{\prime}}$. The right hand side of 4.10 is equal to the completion $\hat{\mathcal{A}}_{\mathcal{I}_{t} \mathcal{A}}$. By the Chinese remainder theorem, $\hat{\mathcal{A}}_{I_{\mathcal{A}} \mathcal{A}} \simeq \oplus_{t^{\prime} \in W_{0} t} \hat{\mathcal{A}}_{j_{t^{\prime}}}$, finishing the proof.

Proposition 4.5. The algebra $\mathcal{H}^{a n}(U)$ is a free $\mathcal{A}^{\text {an }}(U)$ module of rank $\left|W_{0}\right|$, with basis $T_{w} \otimes 1\left(w \in W_{0}\right)$. When $f \in \mathcal{A}^{\text {an }}(U)$ and $s=s_{\alpha}$ with $\alpha \in F_{1}$ we have again the Bernstein-Zelevinski-Lusztig relation

$$
f T_{s}-T_{s} f^{s}=\left(\left(q_{2 \alpha^{\vee}} q_{\alpha \vee}-1\right)+q_{\alpha \vee}^{1 / 2}\left(q_{2 \alpha} \vee-1\right) \theta_{-\alpha / 2}\right) \frac{f-f^{s}}{1-\theta_{-\alpha}} .
$$

This describes the multiplication in the algebra $\mathcal{H}^{\text {an }}(U)$. The center of $\mathcal{H}^{\text {an }}(U)$ is equal to $\mathcal{Z}^{\text {an }}(U)$.

Similarly we have the localized meromorphic affine Hecke algebra $\mathcal{H}^{m e}(U)$, which is defined by

$$
\mathcal{H}^{m e}(U):=\mathcal{F}^{m e}(U) \otimes_{\mathcal{Z}} \mathcal{H},
$$

where $\mathcal{F}^{m e}(U)$ it the quotient field of $\mathcal{Z}^{\text {an }}(U)$. We write $\mathcal{A}^{m e}(U):=$ $\mathcal{F}^{m e}(U) \otimes_{\mathcal{Z}} \mathcal{A}$. It is the ring of meromorphic functions on $U$.

\section{Theorem 4.6.}

$$
\mathcal{H}^{m e}(U)=\oplus_{w \in W_{0}} \mathcal{A}^{m e}(U) \iota_{w}^{0}=\oplus_{w \in W_{0}} \iota_{w}^{0} \mathcal{A}^{m e}(U)
$$

Proof. This is clear from Theorem 4.2 by the remark that $\mathcal{H}^{\text {me }}$ arises from the $\mathcal{F}$-algebra $\mathcal{F} \mathcal{H}$ by extension of scalars according to

$$
\begin{aligned}
\mathcal{H}^{m e}(U) & =\mathcal{F}^{m e}(U) \otimes_{\mathcal{Z}} \mathcal{H} \\
& =\mathcal{F}^{m e}(U) \otimes_{\mathcal{F}}{ }_{\mathcal{F}} \mathcal{H} .
\end{aligned}
$$

\subsection{Lusztig's structure theorem and parabolic induction}

We shall investigate the structure of the tracial states $\chi_{t}$, using Lusztig's technique of localization of $\mathcal{H}$ as discussed above. The results in the present subsection are substitutes for the usual techniques 
of parabolic induction for reductive groups. The results in this subsection are closely related to the results on parabolic induction in the paper [7].

We use in fact a slight variation of the results of Lusztig [26]. There are two main differences. First of all we work with analytic localization at suitably small neighborhoods, instead of Lusztig's use of adic completion. In addition we have replaced the root system of the localized algebra which Lusztig has defined by something slightly different. Lusztig's construction only works with the additional assumption in Convention 2.1 that $f_{s} \in \mathbb{N}$, and this assumption is not natural in our context. We have therefore adapted the construction.

We define a function

$$
T \ni t \rightarrow R_{P(t)} \subset R_{0} \text {, a parabolic subsystem }
$$

by putting $R_{P(t)}:=R_{0} \cap \mathfrak{t}^{*}{ }_{<t>}$, with $\mathfrak{t}^{*}{ }_{<t>} \subset \mathfrak{t}^{*}=\mathbb{R} \otimes_{\mathbb{Z}} X$ the subspace spanned by the roots $\alpha \in R_{0}$ for which one of the following properties holds

(i) $c_{\alpha} \notin \mathcal{O}_{t}^{\times}$(the invertible holomorphic germs at $t$ ).

(ii) $\alpha(t)=1$,

(iii) $\alpha(t)=-1$ and $\alpha \notin 2 X$.

We let $P(t) \subset R_{P(t),+}:=R_{P(t)} \cap R_{0,+}$ be the basis of simple root for $R_{P(t),+}$. We have the following easy consequences of the definition:

Proposition 4.7. $\quad$ (i) $t \rightarrow R_{P(t)}$ is lower semi-continuous with respect to the Zariski-topology of $T$ and the ordering of subsets of $R_{0}$ by inclusion.

(ii) $t \rightarrow R_{P(t)}$ is equivariant: for all $w \in W$ we have $R_{P(w t)}=$ $w\left(R_{P(t)}\right.$.

We denote by $W_{P(t)}$ the parabolic subgroup of $W_{0}$ generated by the reflections $s_{\alpha}$ with $\alpha \in R_{P(t)}$. We say that $t_{1}, t_{2} \in W_{0} t$ are equivalent if there exists a $w \in W_{P\left(t_{1}\right)}$ such that $t_{2}=w\left(t_{1}\right)$. To see that this is actually an equivalence relation, observe that $R_{P\left(t_{2}\right)}=R_{P\left(t_{1}\right)}$ for all $t_{2} \in W_{P\left(t_{1}\right)} t_{1}$. The equivalence classes are the orbits $\varpi=W_{P(t)} t$. This gives a partition of $W_{0} t$ in a collection equivalence classes which are denoted by $\varpi \subset W_{0} t$. If $t \in \varpi$ we sometimes write $P(\varpi), W_{P(\varpi)}$ etc. instead of $P(t), W_{P(t)}$ etc. Note that $W_{0}$ acts transitively on the set of equivalence classes and that for each equivalence class $\varpi, W_{P(\varpi)}$ acts transitively on $\varpi$.

Let $\varpi \subset W_{0} t$ be the equivalence class of $t$. We define:

$$
W_{\varpi}:=\left\{w \in W_{0} \mid w(\varpi)=\varpi\right\} .
$$


ON THE SPECTRAL DECOMPOSITION OF AFFINE HECKE ALGEBRAS 63

By Proposition 4.7 it is clear that $W_{P(\varpi)} \triangleleft W_{\varpi}$, and that this normal subgroup is complemented by the subgroup

$$
W(\varpi):=\left\{w \in W_{\varpi} \mid w(P(\varpi))=P(\varpi)\right\}
$$

Lemma 4.8. For $\alpha \in R_{0}$ we have: $\alpha \in R_{P(\varpi)} \Longleftrightarrow s_{\alpha} \in W_{\varpi}$.

Proof. We only need to show that $s_{\alpha} \in W_{\varpi}$ implies that $\alpha \in R_{P(\varpi)}$ (the other direction being obvious). Notice that if $t \in \varpi$ we have

$$
t^{-1} \varpi \subset \mathbb{Z} R_{P(\varpi)}^{\vee} \otimes \mathbb{C}^{\times}
$$

If $s_{\alpha} \in W_{\varpi}$ then $s_{\alpha}(t) \in \varpi$, and thus

$$
\alpha^{\vee} \otimes \alpha(t) \in \mathbb{Z} R_{P(\varpi)}^{\vee} \otimes \mathbb{C}^{\times} .
$$

By Proposition 4.7, we have $s_{\alpha}\left(R_{P(\varpi)}\right)=R_{P(\varpi)}$. Since $R_{P(\varpi)}$ is parabolic this implies that either $\alpha \in R_{P(\varpi)}$ or that $\alpha\left(R_{P(\varpi)}^{\vee}\right)=0$. In the first case we are done, so let us assume the second case. By (4.19) it follows that $1=\alpha\left(\alpha^{\vee} \otimes \alpha(t)\right)=\alpha(t)^{2}$. If $\alpha(t)=1$ we have $\alpha \in R_{P(\varpi)}$ by definition, contradicting the assumption. If $\alpha(t)=-1$ and $\alpha \notin 2 X$ then, by definition, $\alpha \in R_{P(\varpi)}$, contrary to the assumption. If $\alpha(t)=-1$ and $\alpha=2 x$ for some $x \in X$ then (4.19) implies $1=x\left(\alpha^{\vee} \otimes \alpha(t)\right)=\alpha(t)=-1$, again a contradiction. We conclude that the second case does not arise altogether, and we are done.

Consider the algebra $\mathcal{H}^{P(t)}:=\mathcal{H}\left(X, Y, R_{P(t)}, R_{P(t)}^{\vee}, P(t)\right)$. Note that $W(\varpi)$ acts by means of automorphisms on $\mathcal{R}^{P(t)}=\left(X, Y, R_{P(t)}, R_{(t)}^{\vee}, P(t)\right)$, compatible with the root labels $q$. Thus we may define an action of $\gamma \in W(\varpi)$ on $\mathcal{H}^{P(t)}$ by $\gamma\left(T_{w} \theta_{x}\right)=T_{\left(\gamma w \gamma^{-1}\right)} \theta_{\gamma x}$. In this way we form the algebra $\mathcal{H}^{\varpi}:=\mathcal{H}^{P(t)}[W(\varpi)]$, with its product being defined by $\left(h_{1} \gamma_{1}\right)\left(h_{2} \gamma_{2}\right)=h_{1} \gamma_{1}\left(h_{2}\right) \gamma_{1} \gamma_{2}$.

By Proposition 4.7(i) it is obvious that for any $t \in T$ there exists an open ball $B \subset \mathfrak{t}_{\mathbb{C}}$ centered around the origin such that the following conditions are satisfied:

Conditions 4.9. (i) $\forall \alpha \in R_{0}, b \in B:|\operatorname{Im}(\alpha(b))|<\pi$. In particular, the map exp : $\mathfrak{t}_{\mathbb{C}} \rightarrow T$ restricted to $B$ is an analytic diffeomorphism onto its image $\exp (B)$ in $T$.

(ii) If $w \in W_{0}$ and $t \exp (B) \cap w(t \exp (B)) \neq \emptyset$ then $w t=t$.

(iii) For all $t^{\prime} \in t \exp (B)$, we have $R_{P\left(t^{\prime}\right)} \subset R_{P(t)}$.

Let $t \in T$. We take $B \subset \mathfrak{t}_{\mathbb{C}}$ as above and we put $U=W_{0} t \exp (B)$. Concerning the analytic localization $\mathcal{H}^{a n}(U)$ we have the following analog of Lusztig's first reduction theorem (see [26]): 
Theorem 4.10. For $\varpi \subset W_{0} t$ an equivalence class, we put $U_{\varpi}:=$ $\varpi \exp (B) . \quad$ We define $1_{\varpi} \in \mathcal{A}^{\text {an }}(U)$ by $1_{\varpi}(u)=1$ if $u \in U_{\varpi}$ and $1_{\varpi}(u)=0$ if $u \notin U_{\varpi}$. The elements $1_{\varpi}$ are mutually orthogonal idempotents. Let $t \in \varpi$.

(i) We have $\mathcal{H}^{\varpi, a n}\left(U_{\varpi}\right):=\mathcal{H}^{P(\varpi), a n}\left(U_{\varpi}\right)[W(\varpi)] \simeq 1_{\varpi} \mathcal{H}^{a n}(U) 1_{\varpi}$.

(ii) We can define linear isomorphisms

$$
\Delta_{\varpi_{1}, \varpi_{2}}: \mathcal{H}^{\varpi, a n}\left(U_{\varpi}\right) \rightarrow 1_{\varpi_{1}} \mathcal{H}^{a n}(U) 1_{\varpi_{2}} .
$$

such that $\Delta_{\varpi_{1}, \varpi_{2}}(h) \Delta_{\varpi_{3}, \varpi_{4}}\left(h^{\prime}\right)=\Delta_{\varpi_{1}, \varpi_{4}}\left(h h^{\prime}\right)$ if $\varpi_{2}=\varpi_{3}$, and $\Delta_{\varpi_{1}, \varpi_{2}}(h) \Delta_{\varpi_{3}, \varpi_{4}}\left(h^{\prime}\right)=0$ else.

(iii) The center of $\mathcal{H}^{\varpi, a n}\left(U_{\varpi}\right)$ is $\mathcal{Z}^{\varpi, a n}\left(U_{\varpi}\right):=\left(\mathcal{A}^{a n}\left(U_{\varpi}\right)\right)^{W_{\varpi}}$. This algebra is isomorphic to $\mathcal{Z}^{\text {an }}(U)$ via the map $z \rightarrow 1_{\varpi} z$, and this gives $\mathcal{H}^{\varpi, a n}\left(U_{\varpi}\right)$ the structure of a $\mathcal{Z}^{\text {an }}(U)$-algebra.

(iv) Let $N$ denote the number of equivalence classes in $W_{0} t$. There exists an isomorphism $\mathcal{H}^{a n}(U) \simeq\left(\mathcal{H}^{\varpi, a n}\left(U_{\varpi}\right)\right)_{N}$, the algebra of $N \times N$ matrices with entries in $1_{\varpi} \mathcal{H}^{a n}(U) 1_{\varpi} \simeq \mathcal{H}^{\varpi, a n}\left(U_{\varpi}\right)$. It is an isomorphism of $\mathcal{Z}^{\text {an }}(U)$-algebras.

Proof. The difference with Lusztig's approach is that he works with the $\mathcal{I}_{t}$-adic completions of the algebras instead of the localizations to $U$. In addition, we have a different definition of the root system $R_{P(t)}$.

Using Lemma 4.4 we can copy the arguments of [26], because of the Conditions 4.9 for $B$ and because of Lemma 4.8 (which replaces in our situation Lemma $8.2 \mathrm{~b}$ of [26]). By this we see that the function $c_{\alpha}$ is analytic and invertible on $U_{\varpi} \cup U_{s_{\alpha} \varpi}$ for all $\alpha \in R_{0}$ such that $s_{\alpha} \notin W_{\varpi}$ (compare [26], Lemma 8.9), and this is the crucial point of the construction.

Corollary 4.11. The functor $V \rightarrow V_{\varpi}:=1_{\varpi} V$ defines an equivalence between the category of finite dimensional representations of $\mathcal{H}^{a n}(U)$ and the category of finite dimensional representations of $\mathcal{H}^{\varpi, a n}\left(U_{\varpi}\right)=$ $\mathcal{H}^{P(t), \text { an }}\left(U_{\varpi}\right)[W(\varpi)]$. We have $\operatorname{dim}(V)=N \operatorname{dim}\left(V_{\varpi}\right)$ where $N$ denotes the number of equivalence classes in $W_{0} t$.

Definition 4.12. Let $R_{P} \subset R_{0}$ be a parabolic root subsystem, with $P \subset R_{P,+}:=R_{P} \cap R_{0,+}$ its basis of simple roots. We denote the corresponding parabolic subgroup of $W_{0}$ by $W_{P}:=W\left(R_{P}\right)$. We call

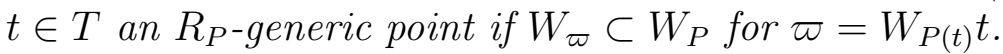

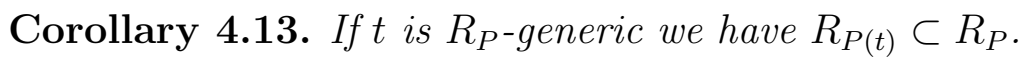

Proof. This is immediate from the definitions. 
We define for any parabolic subsystem $R_{P} \subset R_{0}$ with basis $P$ of $R_{P,+}$ the parabolic subalgebra $\mathcal{H}^{P}=\mathcal{H}\left(X, Y, R_{P}, R_{P}^{\vee}, P\right) \subset \mathcal{H}$ with root labels $q^{P}$.

Assume that $B$ satisfies the Conditions 4.9. Notice that if $t$ is $R_{P^{-}}$

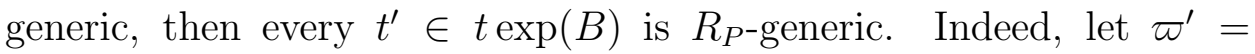
$W_{P\left(t^{\prime}\right)} t^{\prime}$ and $\varpi=W_{P(t)} t$. If $w \in W_{\varpi^{\prime}}$, then there exists a $w^{\prime} \in W_{P\left(t^{\prime}\right)} \subset$ $W_{P(t)}$ (by condition 4.9(iii)) such that $w^{\prime} t^{\prime}=w t^{\prime}$ (since the equivalence

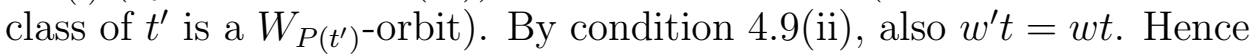
$w \in W_{\varpi} \subset W_{P}$, as required.

We now put $U=W_{0} t \exp (B), U_{P}=W_{P} t \exp (B)$ and consider the localization $\mathcal{H}^{P, \text { an }}\left(U_{P}\right)$.

Corollary 4.14. Assume that $t \in T$ is $R_{P}$-generic. We have $\mathcal{H}^{\text {an }}(U) \simeq$ $\left(\mathcal{H}^{P \text {,an }}\left(U_{P}\right)\right)_{\left|W^{P}\right|}$, where $W^{P}=W_{0} / W_{P}$. Moreover, when we define $1_{P}:=\sum_{\varpi \subset W_{P} t} 1_{\varpi}$ then $\mathcal{H}^{P, \text { an }}\left(U_{P}\right) \simeq 1_{P} \mathcal{H}^{\text {an }}(U) 1_{P}$. These are isomorphisms of $\mathcal{Z}^{\text {an }}(U)$-algebras.

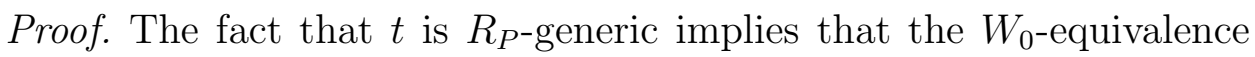
classes of the elements of $W_{P} t$ are equal to the $W_{P}$-equivalence classes of these elements. Therefore we have, by the above theorem, $\mathcal{H}^{P, a n}\left(U_{P}\right) \simeq$ $\left(\mathcal{H}^{\varpi, a n}\left(U_{\varpi}\right)\right)_{n_{P}}$, where $n_{P}$ is the number of equivalence classes $\varpi^{\prime}$ in the orbit $W_{P} t$. And for each $w \in W_{0}, w W_{P} t \subset W_{0} t$ is a union of $n_{P}$ distinct $W_{0}$-equivalence classes. The orbit $W_{0} t$ is the disjoint union of $\left|W^{P}\right|$ subsets of the form $w W_{P} t \subset W_{0} t$, since the stabilizer of $t$ is

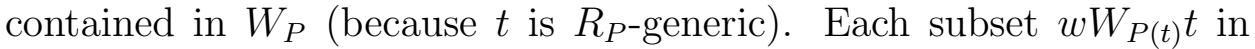
$W_{0} t$ is partitioned into $n_{P}$ equivalence classes, and the result follows.

Recall that, by Proposition 4.3, a finite dimensional representation $(V, \pi)$ of $\mathcal{H}$ with its $\mathcal{Z}$-spectrum contained in $U$ extends uniquely to a representation $\left(V^{a n}, \pi^{a n}\right)$ of $\mathcal{H}^{a n}(U)$.

Corollary 4.15. In the situation of Corollary 4.14, there exists an equivalence $(V, \pi) \rightarrow\left(V_{P}, \pi_{P}\right)$ between $\operatorname{Rep}_{U}(\mathcal{H})$ and $\operatorname{Rep}_{U_{P}}\left(\mathcal{H}^{P}\right)$, characterized by $V_{P}^{a n}=1_{P} V^{a n}$. We have $\operatorname{dim}(V)=\left|W^{P}\right| \operatorname{dim}\left(V_{P}\right)$, and the inverse functor is given by $V_{P} \rightarrow \operatorname{Ind}_{\mathcal{H}^{P}}^{\mathcal{H}}\left(V_{P}\right)=\mathcal{H} \otimes_{\mathcal{H}^{P}} V_{P}$. The character $\chi^{P}$ of the module $\left(V_{P}, \pi_{P}\right)$ of $\mathcal{H}^{P}$ is given in terms of the character $\chi_{\pi}$ of $(V, \pi)$ by the formula $\chi^{P}(h)=\chi_{\pi}\left(1_{P} h\right)$.

Proof. We localize both the algebras $\mathcal{H}$ and $\mathcal{H}^{P}$ and use Proposition 4.3 and Corollary 4.14. Using Corollary 4.14 we see that the functor $\left.V \rightarrow 1_{P} V^{a n}\right|_{\mathcal{H}^{P}}$ is the required equivalence. The relation between the dimensions of $V$ and $V_{P}$ is obvious from this definition. Conversely, 
again using Corollary 4.14, we have

$$
\begin{aligned}
1_{P}\left(\operatorname{Ind}_{\mathcal{H}^{P}}^{\mathcal{H}} V_{P}\right)^{a n} & =1_{P}\left(\mathcal{H} \otimes_{\mathcal{H}^{P}} V_{P}\right)^{a n} \\
& =1_{P}\left(\sum_{P^{\prime}, P^{\prime \prime}} 1_{P^{\prime}} \mathcal{H}^{P, a n}\left(U_{P}\right) 1_{P^{\prime \prime}}\right) \otimes_{\mathcal{H}^{P, a n}\left(U_{P}\right)} 1_{P} V^{a n} \\
& =1_{P} V^{a n}=V_{P}^{a n},
\end{aligned}
$$

finishing the proof.

Proposition 4.16. Let $P \subset F_{0}$ be a subset, and let $R_{P} \subset R_{0}$ be the corresponding standard parabolic subsystem. We define the subtori $T_{P}, T^{P}$ and the lattices $X_{P}, Y_{P}$ as in Proposition 7.3. Put $\mathcal{R}_{P}=$ $\left(X_{P}, Y_{P}, R_{P}, R_{P}^{\vee}, F_{P}\right)$, and let $t \in T^{P}$. There exists a surjective homomorphism $\phi_{t}: \mathcal{H}^{P} \rightarrow \mathcal{H}_{P}$ which is characterized by (1) $\phi_{t}$ is the identity on the finite dimensional Hecke algebra $\mathcal{H}\left(W_{P}\right)$, and (2) $\phi_{t}\left(\theta_{x}\right)=$ $x(t) \theta_{\bar{x}}$, where $\bar{x} \in X_{P}$ is the natural image of $x$ in $X_{P}=X /{ }^{P} X=$ $X /\left(X \cap Y_{P}^{\perp}\right)$.

Proof. We have to check that $\phi_{t}$ is compatible with the BernsteinZelevinski-Lusztig relations. Let $s=s_{\alpha}$ with $\alpha \in P \subset F_{0}$. Then

$$
\begin{aligned}
& \theta_{x} T_{s}-T_{s} \theta_{s(x)}= \\
& \quad=\left\{\begin{array}{cl}
\left(q_{\alpha} \vee-1\right) \frac{\theta_{x}-\theta_{s(x)}}{1-\theta_{-\alpha}} & \text { if } 2 \alpha \notin R_{\mathrm{nr}} . \\
\left(\left(q_{\alpha \vee / 2} q_{\alpha^{\vee}}-1\right)+q_{\alpha \vee / 2}^{1 / 2}\left(q_{\alpha \vee}-1\right) \theta_{-\alpha}\right) \frac{\theta_{x}-\theta_{s(x)}}{1-\theta_{-2 \alpha}} & \text { if } 2 \alpha \in R_{\mathrm{nr}} .
\end{array}\right.
\end{aligned}
$$

Since $s$ acts trivially on $T^{P}$, we have $x(t)=s x(t)$. This implies the result.

Definition 4.17. Let $P \subset F_{0}$ be a subset. In this case we identify the algebra $\mathcal{H}^{P}=\mathcal{H}\left(X, Y, R_{P}, R_{P}^{\vee}, P\right)$ with the subalgebra in $\mathcal{H}$ generated by $\mathcal{H}\left(W_{P}\right)$ and $\mathbb{C}[X]$. Let $(V, \delta)$ be a representation of $\mathcal{H}_{P}$ with central character $W_{P} r \in W_{P} \backslash T_{P}$, and let $t \in T^{P}$. Denote by $\delta_{t}$ the representation $\delta_{t}=\delta \circ \phi_{t}$ of $\mathcal{H}^{P}$. We define a representation $\pi\left(\mathcal{R}_{P}, W_{P} r, \delta, t\right)$ of $\mathcal{H}$ by $\pi\left(\mathcal{R}_{P}, W_{P} r, \delta, t\right)=\operatorname{Ind}_{\mathcal{H}^{P}}^{\mathcal{H}}\left(\delta_{t}\right)$. We refer to such representations as parabolically induced representations.

Corollary 4.18. Let $W_{0} t \in W_{0} \backslash T$, and let $R_{P}$ be a standard parabolic subsystem of $R_{0}$. Suppose that there exists an $r \in T_{P}$ and $t^{P} \in T^{P}$ such

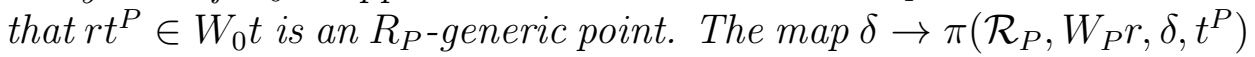
gives an equivalence between the representations of $\mathcal{H}$ with central character $W_{0} t$ and the representations of $\mathcal{H}_{P}$ with central character $W_{P} r$. 
Proof. By Corollary 4.15, the induction functor from representations of $\mathcal{H}^{P}$ to $\mathcal{H}$ gives rise to an equivalence between the representations of $\mathcal{H}^{P}$ with $R_{P}$-regular central character $W_{P} t$ and the representations of $\mathcal{H}$ with central character $W_{0} t$. If $\pi$ is a representation of $\mathcal{H}^{P}$ with central character $W_{P} t$, then it is easy to see that the annihilator of $\pi$ contains the kernel of the homomorphism $\phi_{t^{P}}$. Thus $\pi$ is the lift via $\phi_{t^{P}}$ of a representation $\delta$ of $\mathcal{H}_{P}$. This gives an equivalence between the category of representations of $\mathcal{H}_{P}$ with central character $W_{P} r$ and the representations of $\mathcal{H}^{P}$ with central character $W_{P} t$.

The following proposition describes the induced modules analogous to the "compact realization" of parabolically induced representations of real reductive groups.

Proposition 4.19. Let $(V, \delta)$ be an irreducible representation of $\mathcal{H}_{P}$ with central character $W_{P} r \in W_{P} \backslash T_{P}$ as before. Suppose that $(V, \delta)$ is unitary with respect to an Hermitian inner product $(\cdot, \cdot)$, and that $t \in T_{u}^{P}$. We identify the underlying representation space $V_{\pi}$ of $\pi:=$ $\pi\left(\mathcal{R}_{P}, W_{P} r, \delta, t\right)$ with

$$
V_{\pi}:=\mathcal{H}\left(W^{P}\right) \otimes V
$$

where $\mathcal{H}\left(W^{P}\right) \subset H\left(W_{0}\right)$ denotes the subspace spanned by the elements $T_{w}$ with $w \in W^{P}$. Then $\pi$ is unitary with respect to the Hermitian inner product $\langle\cdot, \cdot\rangle$ defined on $V_{\pi}$ by (with $x, y \in \mathcal{H}\left(W^{P}\right)$, and $\left.u, v \in V\right)$ :

$$
\langle x \otimes u, y \otimes v\rangle:=\tau\left(x^{*} y\right)(u, v) .
$$

Proof. The above form is clearly Hermitian and positive definite. It remains to show that the inner product satisfies

$$
\left\langle\pi(h) m_{1}, m_{2}\right\rangle=\left\langle m_{1}, \pi\left(h^{*}\right) m_{2}\right\rangle
$$

for each $m_{1}, m_{2} \in V_{\pi}, h \in \mathcal{H}$. To this end we recall Theorem 2.20 of [37]. Let $i_{s}: \mathcal{H} \rightarrow \operatorname{End}\left(\mathcal{H}_{0}\right)$ denote the minimal principal series representation induced from $s \in T$. Then the nondegenerate sesquilinear pairing defined on $\mathcal{H}_{0} \times \mathcal{H}_{0}$ by

$$
(x, y):=\tau\left(x^{*} y\right)
$$

satisfies the property

$$
\left(i_{s}(h) x, y\right)=\left(x, i_{s^{*}}\left(h^{*}\right) y\right)
$$

(see Theorem 7.14 for the definition of $s^{*}$ ). We have $\mathcal{H}_{0}=\mathcal{H}\left(W^{P}\right) \otimes$ $\mathcal{H}\left(W_{P}\right)$, and the pairing (4.26) on $\mathcal{H}_{0}$ factors as the tensor product of the pairings on $\mathcal{H}\left(W^{P}\right)$ and on $\mathcal{H}\left(W_{P}\right)$ which are also defined by equation (4.26) but with $x, y$ both in $\mathcal{H}\left(W^{P}\right)$ or both in $\mathcal{H}\left(W_{P}\right)$. 
We choose $r \in T_{P}$ such that $V$ contains a simultaneous eigenvector $v$ for $X_{P}$ with eigenvalue $r$. Via $\delta_{t}$, the vector $v \in V$ has eigenvalue $r t \in T$ with respect to $X$. Thus there is a surjective morphism of $\mathcal{H}^{P}$-modules $\alpha: \mathcal{H}\left(W_{P}\right) \rightarrow V$, where $\mathcal{H}\left(W_{P}\right)$ is the minimal principal series module $i_{r t}^{P}$ for $\mathcal{H}^{P}$, and $V$ is the representation space of $\delta_{t}$. By the above, applied to $\mathcal{H}^{P}$, we have the adjoint injective morphism $\alpha^{*}: V \hookrightarrow \mathcal{H}\left(W_{P}\right)$, where the action on $\mathcal{H}\left(W_{P}\right)$ is via $i_{r^{*} t}^{P}$ (since $(r t)^{*}=r^{*} t$, because $\left.t \in T_{u}^{P}\right)$. By the exactness and the transitivity of induction we get morphisms of $\mathcal{H}$-modules $\operatorname{Ind}(\alpha): i_{r t} \rightarrow \pi$ and $\operatorname{Ind}\left(\alpha^{*}\right): \pi \hookrightarrow i_{r^{*} t}$. Notice that $\operatorname{Ind}(\alpha)=\operatorname{Id}_{\mathcal{H}\left(W^{P}\right)} \otimes \alpha$ and similarly, $\operatorname{Ind}\left(\alpha^{*}\right)=\operatorname{Id}_{\mathcal{H}\left(W^{P}\right)} \otimes \alpha^{*}$. By the factorization of the pairing (4.26) we see that $\operatorname{Ind}(\alpha)$ and $\operatorname{Ind}\left(\alpha^{*}\right)$ are adjoint with respect to the pairings $\langle\cdot, \cdot\rangle$ on $V_{\pi}$ and $(4.26)$ on $\mathcal{H}_{0}$. This, the surjectivity of $\operatorname{Ind}(\alpha)$ and $(4.27)$ gives the desired result (4.25).

Proposition 4.20. With the notations as above, assume that $(V, \delta)$ is a tempered representation with central character $W_{P} r \in W_{P} \backslash T_{P}$ and that $t \in T_{u}^{P}$. Then $\pi:=\pi\left(\mathcal{R}_{P}, W_{P} r, \delta, t\right)$ is a tempered representation of $\mathcal{H}$.

Proof. Recall that we have the identification

$$
V_{\pi}:=\mathcal{H}\left(W^{P}\right) \otimes V
$$

where $\mathcal{H}\left(W^{P}\right) \subset H\left(W_{0}\right)$ denotes the subspace spanned by the elements $T_{w}$ with $w \in W^{P}$. Recall from the proof of Lemma 2.20 that we can find a basis $\left(v_{j}\right)$ of $V$ such that $X_{P}$ acts by upper triangular matrices with respect to this basis. By Casselman's criterion, the diagonal entries are characters $r_{j, j} \in W_{P} r$ of $X_{P}$ which satisfy $\left|x\left(r_{i, i}\right)\right| \leq 1$ for $x \in X_{P}^{+}$. Let $\left(w_{i}\right)$ denote an ordering of the set $W^{P}$ such that the length $l\left(w_{i}\right)$ increases with $i$. We take the tensors $T_{w_{i}} \otimes v_{j}$, ordered lexicographically, as a basis for the representation space of $\pi$. From a direct application of the Bernstein-Zelevinski-Lusztig relations we see that the $\theta_{x}$ are simultaneously upper triangular in this basis, and that the diagonal entries are the elements $w_{i}\left(t r_{j, j}\right)$. Since $w_{i} \in W^{P}, t \in T_{u}^{P}$ and since the vector part of $r_{j, j}$ is an element of the cone generated by the negative roots of $R_{P}^{\vee}$, it follows from a well known characterization of $W^{P}$ that the vector parts of these diagonal entries are in the antidual of the positive chamber. Again using Lemma 2.20 we conclude that $\pi$ is tempered. 
ON THE SPECTRAL DECOMPOSITION OF AFFINE HECKE ALGEBRAS 69

\subsection{The tracial states $\chi_{t}$ and parabolic induction}

In this subsection we will compute the states $\chi_{t}$ on $W_{0}$-orbits of tempered residual cosets of positive dimension in terms of the characters of unitary representations which are induced from discrete series characters of parabolic subalgebras, as was discussed in Subsection 4.1.

Let $L$ be a residual coset such that $R_{L} \subset R_{0}$ is a standard parabolic subsystem. In other words, $F_{L} \subset F_{0}$. Let us denote by $\mathcal{H}_{L}$ the affine Hecke algebra with root datum $\mathcal{R}_{L}:=\left(X_{L}, Y_{L}, R_{L}, R_{L}^{\vee}, F_{L}\right)$ and root labels $q_{L}$. (see Proposition 7.3). Let $r_{L}=c_{L} s_{L} \in T_{L}$ be the corresponding residual point of $\mathcal{R}_{L}$.

Lemma 4.21. Let $U \subset T$ be a nonempty $W_{0}$-invariant open subset. Let $t \in U$. There exists a unique extension of the Eisenstein functional (cf. equations 3.9) $E_{t}$ (which we will also denote by $E_{t}$ ) to the localization $\mathcal{H}^{\text {an }}(U)$, such that $E_{t}(f h)=E_{t}(h f)=f(t) E_{t}(h)$ for all $f \in \mathcal{A}^{a n}(U)$.

Proof. The functional $E_{t}$ factors to a functional of the finite dimensional $\mathbb{C}$-algebra $\mathcal{H}^{t}:=\mathcal{H} / \mathcal{I}_{t} \mathcal{H}$, where $\mathcal{I}_{t}$ is the maximal ideal in $\mathcal{Z}$ corresponding to $W_{0} t$. We have $\mathcal{H} / \mathcal{I}_{t} \mathcal{H}=\mathcal{H}^{a n}(U) / \mathcal{I}_{t}^{a n}(U) \mathcal{H}^{a n}(U)$ for $t \in U$, and this defines the extension with the required property uniquely.

Lemma 4.22. Let $L$ be such that $R_{L} \subset R_{0}$ is a standard parabolic subset of roots, and let $t_{0} \in T$ be $R_{L}$-generic. Set $U=W_{0} t_{0} \exp (B)$ with $B$ satisfying the conditions 4.9 (i), (ii), and (iii). As before, we put $U_{L}=W_{L} t_{0} \exp (B)$.

We denote by $E_{t}^{L}$ the Eisenstein functional of the subalgebra $\mathcal{H}^{L} \subset$ $\mathcal{H}$. For $t_{L} \in T_{L}$ we write $E_{L, t_{L}}$ to denote the Eisenstein functional at $t_{L} \in T_{L}=\operatorname{Hom}\left(X_{L}, \mathbb{C}^{\times}\right)$of the algebra $\mathcal{H}_{L}$. Let $t=t^{L} t_{L} \in U$ with $t^{L} \in T^{L}$ and $t_{L} \in T_{L}$. Recall $1_{L}$ is the characteristic function of $U_{L}$. We have, for all $h \in \mathcal{H}^{L}$ :

(i) $E_{t}\left(1_{L} h 1_{L}\right)=q\left(w^{L}\right) 1_{L}(t) \Delta^{L}(t) E_{t}^{L}(h)$.

(ii) $E_{t}^{L}(h)=E_{L, t_{L}}\left(\phi_{t^{L}}(h)\right)$.

Proof. Because these are both equalities of holomorphic functions of $t \in U$ it suffices to check them for $t$ regular, and outside the union of the residual cosets (in other words, $c(t) c\left(t^{-1}\right) \neq 0$ ).

(i). By the defining properties 3.9 and [37], 2.23(4) we need only to show that the left hand side satisfies the properties $E_{t}\left(1_{L} x h 1_{L}\right)=$ $E_{t}\left(1_{L} h x 1_{L}\right)=t(x) 1_{L}(t) E_{t}(h)$ and $E_{t}\left(1_{L}\right)=q\left(w_{0}\right) 1_{L}(t) \Delta(t)$. These facts follow from Lemma 4.21. 
(ii). We see that

$$
\begin{aligned}
E_{L, t_{L}}\left(\phi_{t^{L}}\left(\theta_{x} h\right)\right) & =E_{L, t_{L}}\left(x\left(t^{L}\right) \theta_{\bar{x}} \phi_{t^{L}}(h)\right) \\
& =x\left(t^{L}\right) \bar{x}\left(t_{L}\right) E_{L, t_{L}}\left(\phi_{t^{L}}(h)\right) \\
& =x(t) E_{L, t_{L}}\left(\phi_{t^{L}}(h)\right),
\end{aligned}
$$

and similarly for $E_{L, t_{L}}\left(\phi_{t^{L}}\left(h \theta_{x}\right)\right)$. The value at $h=1$ is equal to $q\left(w_{L}\right) \Delta_{L}(t)$ on both the left and the right hand side. As in the proof of (i), this is enough to prove the desired equality.

Theorem 4.23. Let $L$ be a residual coset such that $R_{L} \subset R_{0}$ is a standard parabolic subset of roots. Let $t^{L} \in T_{u}^{L}$ be such that $t:=r_{L} t^{L} \in$ $L^{\text {temp }}$ is $R_{L}$-generic. Notice that this condition is satisfied outside a finite union of real codimension one subsets in $T_{u}^{L}$. Let $\Delta_{\mathcal{R}_{L}, W_{L} r_{L}}$ be complete set of inequivalent irreducible representations of the residual algebra $\overline{\mathcal{H}_{L}^{r_{L}}}$, and let $\chi_{\mathcal{H}_{L}, r_{L}}=\sum_{\delta \in \Delta_{\mathcal{R}_{L}, W_{L} r_{L}}} \chi_{\delta} d_{\mathcal{R}_{L}, \delta}$ be the corresponding decomposition in irreducible discrete series characters of the tracial state $\chi_{\mathcal{H}_{L}, r_{L}}$ of $\mathcal{H}_{L}$.

(i) For all $\delta, \pi\left(\mathcal{R}_{L}, W_{L} r_{L}, \delta, t^{L}\right)$ is irreducible, unitary and tempered with central character $W_{0} t$. These representations are mutually inequivalent.

(ii) We have

$$
\left|W^{L}\right| \chi_{t}=\sum_{\delta \in \Delta_{\mathcal{R}_{L}, W_{L} r_{L}}} \chi_{\mathcal{R}_{L}, W_{L} r_{L}, \delta, t^{L}} d_{\mathcal{R}_{L}, \delta},
$$

where $\chi_{\mathcal{R}_{L}, W_{L} r_{L}, \delta, t^{L}}$ denotes the character of $\pi\left(\mathcal{R}_{L}, W_{L} r_{L}, \delta, t^{L}\right)$. In particular, the constants $d_{t, i}$ as in Definition 3.24 are independent of $t$.

(iii) For all (not necessarily $R_{L}$-generic) $t=r_{L} t^{L} \in L^{\text {temp }}$, the character $\chi_{\mathcal{R}_{L}, W_{L} r_{L}, \delta, t^{L}}$ is a positive trace on $\mathcal{H}$. Consequently, the irreducible subrepresentations of $\pi\left(\mathcal{R}_{L}, W_{L} r_{L}, \delta, t^{L}\right)$ extend to $\mathfrak{C}$.

Proof. (i). This is a direct consequence of Corollary 4.18, Proposition 4.19 and Proposition 4.20.

(ii). Recall the definition of the states $\chi_{t}$. Recall that the support of the measure $\nu$ is the union of the tempered residual cosets. We combine Definition 3.16, Proposition 3.15, and Proposition 3.7 to see 
ON THE SPECTRAL DECOMPOSITION OF AFFINE HECKE ALGEBRAS 71

that (with $N_{L}$ the stabilizer of $L$ in $W_{0}$ )

$$
\begin{aligned}
\frac{\left|W_{0}\right|}{\left|N_{L}\right|} \int_{t \in L^{t e m p}} z(t) \chi_{t}(h) d \nu_{L}(t) & \\
& =\sum_{M \in W_{0} L} \int_{t^{M} \in T_{u}^{M}} \int_{t^{\prime} \in t^{M} \epsilon^{M} \xi_{M}} z\left(t^{\prime}\right) E_{t^{\prime}}(h) \eta\left(t^{\prime}\right)
\end{aligned}
$$

for all $h \in \mathcal{H}$ and $z \in \mathcal{Z}$. Rewrite the right hand side as

$$
\frac{k_{L}}{\left|N_{L}\right|} \int_{t^{L} \in T_{u}^{L}} \sum_{w \in W_{0}} J_{w, L}\left(\epsilon^{w L} r_{w L} w\left(t^{L}\right)\right) d^{L}\left(t^{L}\right)
$$

where the inner integrals equal, with $s \in w T^{L}$,

$$
J_{w, L}\left(r_{w L} s\right)=\int_{t^{\prime} \in s \xi_{w L}} z\left(t^{\prime}\right) \frac{E_{t^{\prime}}(h)}{q\left(w_{0}\right) \Delta^{w L}\left(t^{\prime}\right)} m^{w L}\left(t^{\prime}\right) \frac{1}{\Delta_{w L}\left(t^{\prime}\right)} \omega_{w L}\left(t^{\prime}\right),
$$

where

$$
\omega_{w L}(t):=\frac{d_{w L}\left(t_{w L}\right)}{q\left(w_{L}\right) c_{R_{L}}\left(w^{-1} t\right) c_{R_{L}}\left(w^{-1} t^{-1}\right)} .
$$

Hence $J_{w, L}\left(r_{w L} s\right)$ is a linear combination of (possibly higher order) partial derivatives (in the direction of $w T_{L}$ ) of the kernel

$$
z\left(t^{\prime}\right) \frac{E_{t^{\prime}}(h)}{q\left(w_{0}\right) \Delta^{w L}\left(t^{\prime}\right)} m^{w L}\left(t^{\prime}\right)
$$

evaluated at $r_{w L} s$. The $N_{L}$-invariant measure on $L^{\text {temp }}$ on the left hand side of 4.30 is thus obtained by taking the boundary values $\epsilon^{w L} \rightarrow 1$ of the $J_{w, L}\left(\epsilon^{w L} r_{w L} w(t)\right)$, and then sum over the Weyl group as in equation (4.31). Notice that the collection of $R_{L}$-generic points in $L$ is the complement of a union of algebraic subsets of $L$ of codimension $\geq 1$. The kernel (4.34) is regular at such points of $L$. The boundary values at $R_{L}$-generic points are therefore computed simply by specialization at $\epsilon^{w L}=1$. We thus have

$$
z(t) \chi_{t}(h) \bar{\kappa}_{W_{L} L} m_{L}(t)=\frac{k_{L}}{\left|W_{0}\right|} \sum_{w \in W_{0}} J_{w, L}\left(r_{w L} w\left(t^{L}\right)\right) .
$$

The expression on the left hand side can be extended uniquely to $z \in$ $\mathcal{Z}^{a n}(U)$ and $h \in \mathcal{H}^{a n}(U)$. By equation (4.32), each summand in the expression on the right hand can also be extended uniquely to such locally defined analytic $z$ and $h$. 
Take $U=W_{0} t \exp (B)$. We restrict both sides to $1_{L} \mathcal{H}^{L} 1_{L} \subset \mathcal{H}^{L, \text { an }}\left(U_{L}\right)$. Substitute $h$ by $1_{L} h 1_{L}$ with $h \in \mathcal{H}^{L}$. On the left hand side we get, by Corollary 4.15,

$$
\frac{1}{\left|W^{L}\right|} z(t) \chi_{t}^{L}(h) \bar{\kappa}_{W_{L} L} m_{L}(t)
$$

where $\chi_{t}^{L}$ is a central functional on $\mathcal{H}^{L}$, normalized by $\chi_{t}^{L}(e)=1$.

On the other hand, by Lemma 4.22, if $h=1_{L} h 1_{L}$ with $h \in \mathcal{H}^{L}$ then

$$
J_{w, L}\left(w\left(r_{L} t^{L}\right)\right)=\int_{t^{\prime} \in w\left(t^{L}\right) \xi_{w L}} z\left(t^{\prime}\right) \frac{E_{L, t_{L}^{\prime}}\left(\phi_{t^{\prime}, L}(h)\right)}{q\left(w_{L}\right) \Delta_{L}\left(t_{L}^{\prime}\right)} m^{w L}\left(t^{\prime}\right) \omega_{w L}\left(t^{\prime}\right)
$$

if $w\left(r_{L} t^{L}\right) \in U_{L}$, and $J_{w, L}\left(w\left(r_{L} t^{L}\right)\right)=0$ otherwise.

Observe that, because of condition (ii) for $B$, wt $\in U_{L}$ implies that there exists a $w^{\prime} \in W_{L}$ such that $w t=w^{\prime} t$. Since $t=r_{L} t^{L}$ is $R_{L^{-}}$ generic, we see in particular that the stabilizer of $t$ is contained in $W_{L}$. Thus $w t \in U_{L}$ implies that $w \in W_{L}$, and hence that $w t=w\left(r_{L}\right) t^{L}$.

Therefore the sum at the right hand side of equation (4.35) reduces, if $h$ is of the form $1_{L} h 1_{L}$ with $h \in \mathcal{H}^{L}$, to

$$
\frac{k_{L}}{\left|W_{0}\right|} \sum_{w \in W_{L}} \int_{t^{\prime} \in \xi_{w L}} z\left(t^{L} t^{\prime}\right) m^{w L}\left(t^{L} t^{\prime}\right) \frac{E_{L, t^{\prime}}\left(\phi_{t^{L}}(h)\right)}{q\left(w_{L}\right) \Delta_{L}\left(t^{\prime}\right)} \omega_{w L}\left(t^{\prime}\right)
$$

The function $w r_{L} \exp \left(B \cap \mathfrak{t}_{L}\right) \ni t^{\prime} \rightarrow m^{w L}\left(t^{L} t^{\prime}\right)$ is $W_{L}$-invariant on $W_{L} r_{L} \exp \left(B \cap \mathfrak{t}_{L}\right)=\left(t^{L}\right)^{-1} U_{L} \cap T_{L}$, because $m^{L}(t)$ is $W_{L^{-}}$equivariant (i.e. $m^{w L}(w t)=m^{L}(t)$ when $\left.w \in W_{L}\right)$. In other words, this function is in the center of $\mathcal{H}_{L}^{a n}\left(\left(t^{L}\right)^{-1} U_{L} \cap T_{L}\right)$. By Definition 3.16, Corollary 3.20, Definition 3.22, and Theorem 3.25 applied to $\mathcal{H}_{L}$ therefore, this sum reduces to

$$
\frac{\left|W_{L}\right|}{\left|W_{0}\right|} z(t) \chi_{\mathcal{H}_{L}, r_{L}}\left(\phi_{t^{L}}(h)\right) m^{L}(t) \bar{\kappa}_{W_{L} L} m_{\mathcal{R}_{L}, r_{L}}\left(r_{L}\right),
$$

which we can rewrite as

$$
\frac{\left|W_{L}\right|}{\left|W_{0}\right|} z(t) \chi_{\mathcal{H}_{L}, r_{L}}\left(\phi_{t^{L}}(h)\right) \bar{\kappa}_{W_{L} L} m_{L}(t) .
$$

Comparing this with (4.36) we see that, in view of equation (3.67), this implies that for $h \in \mathcal{H}^{L}$,

$$
\left|W^{L}\right| \chi_{t}\left(1_{L} h\right)=\sum_{\delta \in \Delta_{\mathcal{R}_{L}, W_{L} r_{L}}} \chi_{\delta}\left(\phi_{t^{L}}(h)\right) d_{\mathcal{R}_{L}, \delta}=\chi_{t}^{L}(h) .
$$

Applying Corollary 4.15, Definition 4.17, and Corollary 4.18 we obtain (ii).

(iii). By Corollary 3.23, $\chi_{t}$ is $\nu$-almost everywhere a positive trace. On the set of $R_{L}$-generic points $t \in L^{t e m p}$, we have expressed $\chi_{t}$ as 
a positive linear combination of the irreducible induced characters $\chi_{\mathcal{R}_{L}, W_{L} r_{L}, \delta, t^{L}}$. This gives the decomposition of $\chi_{t}$ in terms of irreducible characters of the finite dimensional algebra $\mathcal{H}^{t}:=\mathcal{H} / J_{t}$, where $J_{t}$ denotes the two-sided ideal of $\mathcal{H}$ generated by the maximal ideal $\mathcal{I}_{t}$ of the elements of the center $\mathcal{Z}$ which vanish at $W_{0} t$.

On the other hand, we have the decomposition of $\chi_{t}$ in irreducible characters of the finite dimensional Hilbert algebra $\overline{\mathcal{H}^{t}}$, as in Definition 3.24. This algebra is a quotient algebra of $\mathcal{H}^{t}$. Because $\mathcal{H}^{t}$ is finite dimensional, there is no distinction between topological and algebraic irreducibility. We therefore have two decompositions of $\chi_{t}$ in terms of irreducible characters of $\mathcal{H}^{t}$. The irreducible characters of $\mathcal{H}^{t}$ are linearly independent, and thus the two decompositions are necessarily the same. This implies that the induced characters $\chi_{\mathcal{R}_{L}, W_{L} r_{L}, \delta, t^{L}}$ are characters of the Hilbert algebra $\overline{\mathcal{H}^{t}}$. In particular, the characters are positive traces for all $R_{L_{\text {-generic }}} t \in L^{t e m p}$. These induced characters are regular functions of $t \in L^{t e m p}$. Hence by continuity, they are positive traces for all $t^{L}$.

By Corollary 2.17(i), $\chi\left(\mathcal{R}_{L}, W_{L} r_{L}, \delta, t^{L}\right)$ extends to a continuous trace of $\mathfrak{C}$ for all $t \in L^{t e m p}$. According to the construction in [15], Paragraphe 6.6. this character is the trace of a (obviously finite dimensional) representation of $\mathfrak{C}$, quasi-equivalent with $\pi\left(\mathcal{R}_{L}, W_{L} r_{L}, \delta, t^{L}\right)$ when restricted to $\mathcal{H}$. Hence all subrepresentations of $\pi\left(\mathcal{R}_{L}, W_{L} r_{L}, \delta, t^{L}\right)$ extend to $\mathfrak{C}$.

Corollary 4.24. For all $x \in \mathcal{H}$, the $\nu_{L}$-measurable function $L^{\text {temp }} \ni$ $t \rightarrow \chi_{t}(x)$ can be defined by the restriction to $L^{\text {temp }}$ of a regular function on $L$. For $x \in \mathfrak{C}$, the function $t \rightarrow \chi_{t}(x)$ is continuous on $L^{\text {temp }}$.

Proof. The first assertion was shown in the proof of Theorem 4.23(iii). If $x \in \mathfrak{C}$ there exists a sequence $\left(x_{i}\right)$ with $x_{i} \rightarrow x$ and $x_{i} \in \mathcal{H}$. By Corollary 2.17(i), the function $t \rightarrow \chi_{t}(x)$ is a uniform limit of the functions $t \rightarrow \chi_{t}\left(x_{i}\right)$, proving the continuity.

The next Theorem basically is the Plancherel decomposition of $\mathfrak{H}$. (In the next subsection we will refine the formula by adding more details about the spectrum of $\mathfrak{C}$.)

Theorem 4.25. We have the following isomorphism of Hilbert spaces:

$$
\mathfrak{H}=\int_{W_{0} \backslash T}^{\oplus} \overline{\mathcal{H}^{t}}\left|W_{0} t\right| d \nu(t) .
$$

The support of the probability measure $\nu$ is the union of the tempered residual cosets. If $t=r_{L} t^{L} \in L^{\text {temp }}$ is $R_{L}$-generic, then the residue 
algebra $\overline{\mathcal{H}^{t}}$ has the structure

$$
\overline{\mathcal{H}^{t}} \simeq\left(\overline{\mathcal{H}_{L}^{r_{L}}}\right)_{\left|W^{L}\right|}
$$

Finally, the residue algebra $\overline{\mathcal{H}^{r}}$ at a residual point $r \in T$ is of the form

$$
\overline{\mathcal{H}^{r}}=\bigoplus_{\delta \in \Delta_{\mathcal{R}, W_{0} r}} \operatorname{End}\left(V_{\delta}\right)
$$

with the Hermitian form on the summand $\operatorname{End}\left(V_{\delta}\right)$ given by

$$
(A, B)=d_{\delta} \operatorname{trace}\left(A^{*} B\right)
$$

where the positive real numbers $d_{\delta}$ are defined as in Definition 3.24 (with the notational convention that $d_{\delta}=d_{r, i}$ if $\delta=\delta_{i}$ is the irreducible representation of $\overline{\mathcal{H}^{r}}$ corresponding to the central idempotent $\left.e_{i}\right)$.

Proof. The Hilbert space $\mathfrak{H}$ is the completion of $\mathcal{H}$ with respect to the positive trace $\tau$. In Corollary 3.23(v) we have written $\tau$ as a positive superposition of positive traces $\chi_{t}$, with $t \in W_{0} \backslash T$. In Theorem 4.23 we established that, outside a set of measure zero, $\chi_{t}$ is a finite linear combination of traces of irreducible representations $\pi_{\mathcal{R}_{L}, W_{L} r_{L}, \delta, t^{L}}$ which extend to $\mathfrak{C}$. Thus Corollary 3.23(v) is a positive decomposition of $\tau$ in terms of traces of elements of $\hat{\mathfrak{C}}$. This allows us to apply 8.8.5 and 8.8.6 of [15] in order to obtain (4.42). The statements about the residual algebra of a residual point follow directly from the Definition 3.24. Finally, equation (4.43) follows from Theorem 4.23 in combination with the factorization Proposition 4.19 of the inner product $\langle\cdot, \cdot\rangle$ on the induced representations.

\subsection{The generic residue of the Hecke algebra}

In this subsection we will use Theorem 4.23 in order to compute explicitly the local traces $\chi_{t}$ when $t=r_{L} t^{L}$ is an $R_{L}$-generic element of $L^{\text {temp }}$. Here we assume that $L$ is a residual coset such that $R_{L}$ is a standard parabolic subset of $R_{0}$ with basis $F_{L}$ of simple roots. Since we assume that $t$ is $R_{L}$-generic, we have $P(t)=F_{L}$. As before, we put $W_{L} t=\varpi$, the equivalence class of $t$. By the genericity of $t, W(\varpi)=1$.

Observe that the residual algebra $\overline{\mathcal{H}^{t}}$ (see Definition 3.24) is of the form $\overline{\mathcal{H}^{t}}=\mathcal{H}^{t} / \operatorname{Rad}_{t}$, where $\mathcal{H}^{t}$ is the quotient algebra $\mathcal{H}^{t}:=\mathcal{H} / \mathcal{I}_{t} \mathcal{H}$ (with $\mathcal{I}_{t}$ the maximal ideal of $\mathcal{Z}$ corresponding to $t$ ), and where $\operatorname{Rad}_{t}$ is the radical of the positive semi-definite form $(x, y)_{t}:=\chi_{t}\left(x^{*} y\right)$ (viewed as a form on $\mathcal{H}^{t}$ ). 
By Lusztig's Structure Theorem 4.10, $\mathcal{H}^{t}$ has the following decomposition in the case where $t$ is $R_{L}$-generic:

$$
\mathcal{H}^{t}=\bigoplus_{u, v \in W^{L}} \iota_{u}^{0} e_{\varpi} \mathcal{H}^{L} \iota_{v^{-1}}^{0}
$$

where $e_{\varpi}=1_{\varpi} \bmod \left(\mathcal{I}_{t} \mathcal{H}^{a n}(U)\right)$, the image of $1_{\varpi}$ in $\mathcal{H}^{t}$ (in the notation of Section 4). We remark that this is not an orthogonal decomposition with respect to $(x, y)_{t}$.

The subspace $\iota_{u}^{0} e_{\varpi} \mathcal{H}^{L} \iota_{v^{-1}}^{0}$ is equal to $e_{u \varpi} \mathcal{H}^{t} e_{v \varpi}$. If $u=v$ then this is a subalgebra of $\mathcal{H}^{t}$. If $u=v=e$ then this subalgebra is isomorphic to $\mathcal{H}^{L, t}$ via the isomorphism $\mathcal{H}^{L, t} \ni x \rightarrow e_{\varpi} x \in e_{\varpi} \mathcal{H}^{L}$.

Let $P, Q \subset F_{0}$ be subsets. We denote by $W(P, Q)$ the following set of Weyl group elements: $W(P, Q):=\left\{w \in W_{0} \mid w(P)=Q\right\}$. If $P=Q \subset F_{0}$ then we simply write $W(P)=W(P, P)$

Let $n \in W_{0}$ be such that $n\left(F_{L}\right)=F_{M} \subset F_{0}$, in other words, let $n \in W\left(F_{L}, F_{M}\right)$. Then the map

$$
\begin{aligned}
\Delta_{n \varpi, n \varpi}: e_{\varpi} \mathcal{H}^{L} & \rightarrow e_{n \varpi} \mathcal{H}^{M} \\
x & \rightarrow \iota_{n}^{0} x \iota_{n^{-1}}^{0}
\end{aligned}
$$

is an isomorphism. By the results of Lusztig [26], section 8, it satisfies (with $h \in \mathcal{H}^{L}$ ):

$$
\Delta_{n \varpi, n \varpi}\left(e_{\varpi} h\right)=e_{n \varpi} \psi_{n}(h),
$$

where $\psi_{n}: \mathcal{H}^{L} \rightarrow \mathcal{H}^{M}$ is the isomorphism of algebras defined by (with $w \in W_{L}$ and $\left.x \in X\right)$ :

$$
\psi_{n}\left(T_{w}\right)=T_{n w n^{-1}}, \psi_{n}\left(\theta_{x}\right)=\theta_{n x} .
$$

Recall that Theorem 4.23(ii) implies that for all $h \in \mathcal{H}^{L}$,

$$
\left|W^{L}\right| \chi_{t}\left(e_{\varpi} h\right)=\chi_{\mathcal{H}_{L}, r_{L}}\left(\phi_{t^{L}}(h)\right) .
$$

Corollary 4.26. Write $n(t)=s=r_{M}^{\prime} s^{M}$.

$$
\chi_{\mathcal{H}_{M}, r_{M}^{\prime}}\left(\phi_{s^{M}}\left(\psi_{n}(h)\right)\right)=\chi_{\mathcal{H}_{L}, r_{L}}\left(\phi_{t^{L}}(h)\right) .
$$

(ii) Let $\Psi_{n}: \Delta_{\mathcal{R}_{L}, W_{L} r_{L}} \rightarrow \Delta_{\mathcal{R}_{M}, W_{M} r_{M}^{\prime}}$ be the bijection such that $\Psi_{n}(\delta) \simeq \delta \circ \psi_{n}^{-1}$. Then $\Psi_{n}$ respects the residual degree: $d_{\mathcal{R}_{L}, \delta}=$ $d_{\mathcal{R}_{M}, \Psi_{n}(\delta)}$.

Proof. (i) This follows from the above text, and the fact that $\chi_{t}$ is a central functional.

(ii) It is clear from Casselman's criteria that $\Psi_{n}$ indeed defines a bijection between the sets of discrete series representations $\Delta_{\mathcal{R}_{L}, W_{L} r_{L}}$ and $\Delta_{\mathcal{R}_{M}, W_{M} r_{M}^{\prime}}$. The result follows from (i) and Theorem 4.23(ii). 
Proposition 4.27. For all $h, g \in \mathcal{H}^{L}$ we have

$$
\left|W^{L}\right| m^{L}(t) \chi_{t}\left(\left(e_{\varpi} h\right)^{*}\left(e_{\varpi} g\right)\right)=\chi_{\mathcal{H}_{L}, r_{L}}\left(\phi_{t^{L}}\left(h^{\sharp} g\right)\right),
$$

where $\sharp$ denotes the $*$-operator of $\mathcal{H}^{L}$ (thus $T_{w}^{\sharp}=T_{w^{-1}}$ if $w \in W_{L}$, and $\theta_{x}^{\sharp}=T_{w_{L}} \theta_{-w_{L} x} T_{w_{L}}^{-1}$ where $w_{L}$ denotes the longest element of $\left.W_{L}\right)$.

Proof. This will be proved by the computation in the proof of Theorem 4.30.

Corollary 4.28. We equip $e_{\varpi} \mathcal{H}^{t} e_{\varpi}$ with the positive semi-definite sesquilinear pairing obtained by restriction of the pairing $\left|W^{L}\right| m^{L}(t)(x, y)_{t}=$ $\left|W^{L}\right| m^{L}(t) \chi_{t}\left(x^{*} y\right)$ defined on $\mathcal{H}^{t}$. The residual algebra $\overline{\mathcal{H}^{L, t}}$ is isomorphic as a Hilbert algebra to the quotient of $e_{\varpi} \mathcal{H}^{t} e_{\varpi}$ by the radical of this pairing, via the map $x \rightarrow e_{\varpi} x$.

Corollary 4.29. Recall the notation of Proposition 4.19. We consider $V_{\pi}$ as a module over $\mathcal{H}^{t}$. Put $V_{\pi, \varpi}=\pi\left(e_{\varpi}\right)\left(V_{\pi}\right)$. Choose an isometric embedding $\bar{i}: V \rightarrow \overline{\mathcal{H}_{L}^{r_{L}}}$ (as $\mathcal{H}_{L}$-modules). Let $\bar{j}$ denote the unique module map $\bar{j}: V_{\pi} \rightarrow \overline{\mathcal{H}}^{t}$ such that $\bar{j}(1 \otimes v)=e_{\varpi}\left(\bar{\phi}_{t^{L}}\right)^{-1}(\bar{i}(v))$, where $\bar{\phi}_{t^{L}}: \overline{\mathcal{H}^{L, t}} \rightarrow \overline{\mathcal{H}_{L}^{r_{L}}}$ denotes the isometric isomorphism determined by the homomorphism $\phi_{t^{L}}$ (cf. Proposition 4.16). For any $v \in V$ we denote by $i(v)$ any lift of $\bar{i}(v)$, and similarly for $j$. We have:

(i) $V_{\pi, \varpi}=1 \otimes V$, and $\bar{j}: V_{\pi, \varpi} \stackrel{\sim}{\rightarrow} e_{\varpi}\left(\bar{\phi}_{t^{L}}\right)^{-1}(\bar{i}(V))$.

(ii) The positive definite Hermitian form $\langle\cdot, \cdot\rangle$ on $V_{\pi}$ (see Proposition 4.19) can be expressed by:

$$
\langle v, w\rangle=\left|W^{L}\right| m^{L}(t) \chi_{t}\left(j(v)^{*} j(w)\right) .
$$

Proof. (i) is straightforward by observing that $\phi_{t^{L}}$ descends to $\mathcal{H}^{L, t}$ and so defines an isometric isomorphism $\bar{\phi}_{t^{L}}$ by (4.50), applied to $\mathcal{R}^{L}$ instead of $\mathcal{R}$ (thus $e_{\omega}=1$, and $\left|W^{L}\right|=1$ ). Since $V_{\pi}$ is irreducible, it is enough to compare the inner products on $V_{\pi, \varpi}$ in order to prove (ii). Apply Proposition 4.27 and Corollary 4.28.

Assume that $R_{M}$ and $R_{L}$ are associate standard parabolic subsystems. Let $\varpi_{1}=\varpi$ and $\varpi_{2}$ be equivalence classes inside $W_{0} t$ such that $\varpi_{1}=W_{L} t$ and $\varpi_{2}=W_{M} s$.

Theorem 4.30. Let $n \in W\left(F_{M}, F_{L}\right)$ be such that $n\left(\varpi_{2}\right)=\varpi_{1}$. Let $h \in \mathcal{H}^{L}$ and $h^{\prime} \in \mathcal{H}^{M}$ such that $e_{\varpi_{2}} h^{\prime}=\iota_{n^{-1}}^{0} e_{\varpi_{1}} h \iota_{n}^{0} \in e_{\varpi_{2}} \mathcal{H}^{M}$. We have

$$
\begin{aligned}
\chi_{t}\left(\left(h e_{\varpi_{1}} \iota_{n}^{0}\right)^{*}\left(h e_{\varpi_{1}} \iota_{n}^{0}\right)\right) & =\chi_{t}\left(\left(h e_{\varpi_{1}}\right)^{*}\left(h e_{\varpi_{1}}\right)\right) \\
& =\chi_{t}\left(\left(h^{\prime} e_{\varpi_{2}}\right)^{*}\left(h^{\prime} e_{\varpi_{2}}\right)\right)
\end{aligned}
$$


ON THE SPECTRAL DECOMPOSITION OF AFFINE HECKE ALGEBRAS 77

Proof. Before we embark on this computation we establish some useful relations. First recall that (Theorem 7.14) $t^{*}:=\overline{t^{-1}} \in W_{L} t$. Also recall Proposition 2.9. We see that

$$
\begin{aligned}
e_{\varpi_{1}}^{*} & =T_{w_{0}} e_{w_{0} \varpi_{1}} T_{w_{0}}^{-1} \\
& =T_{w^{L}} e_{w^{L} \varpi_{1}} T_{w^{L}}^{-1},
\end{aligned}
$$

where $w^{L}$ denotes the longest element of set of minimal coset representatives $W^{L}$. Next we observe that for any $w \in W_{0}$,

$$
\left(\iota_{w}^{0}\right)^{*}=T_{w_{0}}\left(\prod_{\substack{\alpha>0 \\ w^{\prime}(\alpha)<0}}\left(\frac{c_{\alpha}}{c_{-\alpha}}\right) \iota_{w^{\prime-1}}^{0}\right) T_{w_{0}}^{-1},
$$

where $w^{\prime}:=w_{0} w w_{0}$. This formula follows in a straightforward way from Definition (4.6).

If $s$ is a simple reflection and $\varpi \subset W_{0} t$ an equivalence class, we check that (recall that $t$ is $R_{L}$-generic)

$$
e_{s \varpi} T_{s} e_{\varpi}= \begin{cases}e_{\varpi} T_{s} & \text { if } s \varpi=\varpi \\ e_{s \varpi} q(s) c_{\alpha} \iota_{s}^{0} & \text { else. }\end{cases}
$$

Since we assume that $t$ is $R_{L}$-generic, we have $u\left(\varpi_{1}\right) \neq w\left(\varpi_{1}\right)$ for all $w \in W^{L}$ and all $u \in W_{0}$ such that $l(u)<l(w)$. From this, (4.56) and induction to the length of $l(w)$ we see that

$$
\begin{aligned}
e_{w \varpi_{1}} T_{w} e_{\varpi_{1}} & =q(w)\left(\prod_{\substack{\alpha>0 \\
w^{-1}(\alpha)<0}} c_{\alpha}\right) \iota_{w}^{0} e_{\varpi_{1}} \\
& =e_{w \varpi_{1}} q(w)\left(\prod_{\substack{\alpha>0 \\
w^{-1}(\alpha)<0}} c_{\alpha}\right) \iota_{w}^{0}
\end{aligned}
$$

for all $w \in W^{L}$. Observe that we also have

$$
e_{w \varpi_{1}} T_{w^{-1}}^{-1} e_{\varpi_{1}}=\left(\prod_{\substack{\alpha>0 \\ w^{-1}(\alpha)<0}} c_{\alpha}\right) \iota_{w}^{0} e_{\varpi_{1}} .
$$

We note that $w_{0}=w^{L} w_{L}$. Since $w_{L}$ and $w_{0}$ are involutions, this implies that $\left(w^{L}\right)^{-1}=w^{L^{\prime}}$, where $R_{L^{\prime}}=w_{0}\left(R_{L}\right)$ (also a standard parabolic subsystem).

Let $h=T_{w} \theta_{x} \in \mathcal{H}^{L}$, with $w \in W_{L}$ and $x \in X$. Keeping in mind the above preliminary remarks, and the fact that $\chi_{t}$ is central, we now 
compute (with $x^{\prime}:=-w_{0}(x)$ and $w_{0} \varpi_{1}=\varpi_{1}^{\prime}$ ):

$$
\begin{aligned}
& \chi_{t}\left(\left(h e_{\varpi_{1}} \iota_{n}^{0}\right)^{*}\left(h e_{\varpi_{1}} \iota_{n}^{0}\right)\right) \\
& =\chi_{t}\left(T_{w_{0}} \prod_{\substack{\alpha>0 \\
n^{\prime}(\alpha)<0}}\left(\frac{c_{\alpha}}{c_{-\alpha}}\right) \iota_{n^{\prime-1}}^{0} e_{\varpi_{1}^{\prime}} \theta_{x^{\prime}} T_{w_{0}}^{-1} T_{w^{-1}} T_{w} \theta_{x} e_{\varpi_{1}} \iota_{n}^{0}\right) \\
& =\chi_{t}\left(T_{w^{M^{\prime}}} \prod_{\substack{\alpha>0 \\
n^{\prime}(\alpha)<0}}\left(\frac{c_{\alpha}}{c_{-\alpha}}\right) \iota_{n^{\prime-1}}^{0} e_{\varpi_{1}^{\prime}} \theta_{x^{\prime}} T_{w^{L^{\prime}}}^{-1} T_{w_{L}}^{-1} T_{w^{-1}} T_{w} \theta_{x} e_{\varpi_{1}} \iota_{n}^{0} T_{w_{M}}\right) \\
& =\chi_{t}\left(e_{\varpi_{2}} T_{w^{M^{\prime}}} e_{\varpi_{2}^{\prime}}\left(\prod_{\substack{\alpha>0 \\
w^{M^{\prime}}(\alpha)<0}} c_{\alpha}\right)\left(\prod_{\substack{\alpha>0 \\
w^{\prime}(\alpha)<0}} c_{n^{\prime-1} \alpha}^{-1}\right)\right. \\
& \left.\iota_{n^{\prime-1}}^{0} \theta_{x^{\prime}} e_{\varpi_{1}^{\prime}} T_{\left(w^{L}\right)^{-1}}^{-1} e_{\varpi_{1}} T_{w_{L}}^{-1} T_{w^{-1}} T_{w} \theta_{x} e_{\varpi_{1}} \iota_{n}^{0} T_{w_{M}}\right) \\
& =q\left(w^{M^{\prime}}\right) \chi_{t}\left(e_{\varpi_{2}} \iota_{w^{M^{\prime}}}^{0}\left(\prod_{\substack{\alpha>0 \\
w^{M^{\prime}} \alpha<0}} c_{-\alpha}\right)\left(\prod_{\substack{\alpha>0 \\
w^{\prime}(\alpha)<0}} c_{\alpha}\right)\left(\prod_{\substack{\alpha>0 \\
L^{L^{\prime}}(\alpha)<0}} c_{n^{\prime-1} \alpha}^{-1}\right)\right. \\
& \left.\iota_{n^{\prime-1}}^{0} \theta_{x^{\prime}}\left(\prod_{\substack{\alpha>0 \\
w^{\prime}{ }_{\alpha<0}}} c_{\alpha}\right) \iota_{w^{L}}^{0} T_{w_{L}}^{-1} T_{w^{-1}} T_{w} \theta_{x} e_{\varpi_{1}} \iota_{n}^{0} T_{w_{M}}\right) \\
& =q\left(w^{L}\right) \chi_{t}\left(e_{\varpi_{2}} \iota_{n^{-1}}^{0} \iota_{w^{L^{\prime}}}^{0}\left(\prod_{\substack{\alpha>0 \\
w^{M^{\prime}} \alpha<0}} c_{-n^{\prime} \alpha}\right)\left(\prod_{\substack{\alpha>0 \\
w^{M^{\prime}}(\alpha)<0}} c_{n^{\prime} \alpha}\right)\left(\prod_{\substack{\alpha>0 \\
w^{L^{\prime}}(\alpha)<0}} c_{\alpha}^{-1}\right)\right. \\
& \left.\left(\prod_{\substack{\alpha>0 \\
w^{\prime} \alpha<0}} c_{\alpha}\right) \theta_{x^{\prime}} \iota_{w^{L}}^{0} T_{w_{L}}^{-1} T_{w^{-1}} T_{w} \theta_{x} e_{\varpi_{1}} \iota_{n}^{0} T_{w_{M}}\right) \\
& =q\left(w^{L}\right) \chi_{t}\left(e_{\varpi_{2}} \iota_{n^{-1}}^{0}\left(\prod_{\substack{\alpha>0 \\
w^{M^{\prime}} \alpha<0}} c_{-w^{L^{\prime}} n^{\prime} \alpha}\right)\left(\prod_{\substack{\alpha>0 \\
w^{M^{\prime}}(\alpha)<0}} c_{w^{L^{\prime}} n^{\prime} \alpha}\right)\right. \\
& \left.\theta_{-w_{L} x} T_{w_{L}}^{-1} T_{w^{-1}} T_{w} \theta_{x} e_{\varpi_{1}} \iota_{n}^{0} T_{w_{M}}\right) \\
& =q\left(w^{L}\right) \chi_{t}\left(e_{\varpi_{1}}\left(\prod_{\alpha \notin R_{M}} c_{n \alpha}\right) e_{\varpi_{1}} \theta_{-w_{L} x} T_{w_{L}}^{-1} T_{w^{-1}} T_{w} \theta_{x} T_{w_{L}}\right) \\
& =q\left(w^{L}\right)\left(\prod_{\alpha \notin R_{L}} c_{\alpha}(t)\right) \chi_{t}\left(e_{\varpi_{1}} T_{w_{L}} \theta_{-w_{L} x} T_{w_{L}}^{-1} T_{w^{-1}} T_{w} \theta_{x}\right) \\
& =m^{L}(t)^{-1} \chi_{t}\left(e_{\varpi_{1}} h^{\sharp} h\right) \\
& =\left|W^{L}\right|^{-1} m^{L}(t)^{-1} \chi_{\mathcal{H}_{L}, r_{L}}\left(\phi_{t^{L}}\left(h^{\sharp} h\right)\right) \text {. }
\end{aligned}
$$

The result is independent of $n$, implying the first equality of the theorem. The second equality follows because $\chi_{t}$ is central. Indeed, this 
ON THE SPECTRAL DECOMPOSITION OF AFFINE HECKE ALGEBRAS 79

implies that we have

$$
\chi_{t}\left(e_{\varpi_{1}} h^{\sharp} h\right)=\chi_{t}\left(e_{\varpi_{2}}\left(h^{\prime}\right)^{\sharp} h^{\prime}\right),
$$

where the second $\sharp$ of course refers to the $*$-structure on $\mathcal{H}^{M}$.

In equation (4.59) we have used the evaluation

$$
\left(\prod_{\alpha \notin R_{L}} c_{\alpha}\right) e_{\varpi_{1}}=\left(\prod_{\alpha \notin R_{L}} c_{\alpha}(t)\right) e_{\varpi 1} .
$$

This is allowed because the element

$$
\left(\prod_{\alpha \notin R_{L}} c_{\alpha}\right) \in \mathcal{A}
$$

is $W_{L}$-invariant, and thus belongs to the center of $\mathcal{H}^{L}$.

At several places in equations (4.59) and (4.60) we have freely used formulae of Lusztig [26] (see Theorem 4.10) for the structure of $\mathcal{H}^{t}$. For example,

$$
\iota_{n^{-1}}^{0} e_{\varpi_{1}} h \iota_{n}^{0}=e_{\varpi_{2}} \psi_{n^{-1}}(h)=e_{\varpi} h^{\prime}
$$

when $h \in \mathcal{H}^{L}$. By this we easily see that for all $h \in \mathcal{H}^{L}$,

$$
\iota_{n^{-1}}^{0} e_{\varpi_{1}} h^{\sharp} \iota_{n}^{0}=e_{\varpi_{2}}\left(h^{\prime}\right)^{\sharp},
$$

and hence we may conclude by equation (4.59) that

$$
\chi_{t}\left(\left(h e_{\varpi_{1}}\right)^{*}\left(h e_{\varpi_{1}}\right)\right)=\chi_{t}\left(\left(h^{\prime} e_{\varpi_{2}}\right)^{*}\left(h^{\prime} e_{\varpi_{2}}\right)\right) .
$$

The proof is finished.

Corollary 4.31. Let $L, M_{1}, M_{2}$ be residual cosets such that $F_{L}, F_{M_{1}}$ and $F_{M_{2}}$ are associate subsets of $F_{0}$, and let $n_{i} \in W\left(L, M_{i}\right)(i=1,2)$. The map

$$
\begin{aligned}
\Delta_{n_{1} \varpi, n_{2} \varpi}: e_{\varpi} \mathcal{H}^{L} & \rightarrow \iota_{n_{1}}^{0} e_{\varpi} \mathcal{H}^{L} \iota_{n_{2}^{-1}}^{0}=e_{n_{1} \varpi} \mathcal{H} e_{n_{2} \varpi} \\
x & \rightarrow \iota_{n_{1}}^{0} x \iota_{n_{2}^{-1}}^{0}
\end{aligned}
$$

is a partial isometry with respect to the natural positive semi-definite pairing on $\mathcal{H}^{t}$ given by $(x, y)_{t}:=\chi_{t}\left(x^{*} y\right)$.

Proof. We have, with $\varpi^{\prime}:=n_{1} \varpi, \Delta_{n_{1} \varpi, n_{2} \varpi}=\Delta_{\varpi^{\prime}, n_{2} n_{1}^{-1} \varpi^{\prime}}^{\varpi^{\prime}} \circ \Delta_{n_{1} \varpi, n_{1} \varpi}$, where $\Delta_{\varpi^{\prime}, n_{2} n_{1}^{-1} \varpi^{\prime}}^{\varpi^{\prime}}$ is defined by

$$
\begin{aligned}
\Delta_{\varpi^{\prime}, n_{2} n_{1}^{-1} \varpi^{\prime}}^{\varpi^{\prime}}: e_{\varpi^{\prime}} \mathcal{H}^{M_{1}} & \rightarrow e_{\varpi^{\prime}} \mathcal{H}^{M_{1}} \iota_{n_{1} n_{2}^{-1}}^{0} \\
x & \rightarrow x \iota_{n_{1} n_{2}^{-1}}^{0}
\end{aligned}
$$

Both these respect the pairing $(\cdot, \cdot)_{t}$, by Theorem 4.30 . 


\subsection{Unitarity and regularity of intertwining operators}

Let $L, M$ be associate residual subspaces such that $R_{L}, R_{M}$ are standard parabolic subsystems of $R_{0}$. Let $n \in W_{0}$ be such that $n\left(R_{L,+}\right)=$ $R_{M,+}$. As before we let $\psi_{n}: \mathcal{H}^{L} \rightarrow \mathcal{H}^{M}$ denote the isomorphism defined by $\psi_{n}\left(T_{w}\right)=T_{n w n^{-1}}$ and $\psi_{n}\left(\theta_{x}\right)=\theta_{n x}$. Let $(V, \delta)$ be an irreducible discrete series representation of $\mathcal{H}_{L}^{r_{L}}$ and let $t=r_{L} t^{L}$ be an $R_{L}$-generic point of $r_{L} T^{L}$. Let $s=n(t)=r_{M}^{\prime} s^{M}$, and let $\left(V^{\prime}, \delta^{\prime}\right)$ be a realization of the discrete series representation $\delta^{\prime}=\Psi_{n}(\delta)$.

Choose a unitary isomorphism $\tilde{\delta}: V \rightarrow V^{\prime}$ such that

$$
\tilde{\delta}\left(\delta_{t}(h) v\right)=\delta_{s}^{\prime}\left(\psi_{n}(h)\right)(\tilde{\delta}(v)) .
$$

Recall that $V_{\pi}$ with $\pi=\pi\left(\mathcal{R}_{L}, W_{L} r_{L}, \delta, t^{L}\right)$ is isomorphic to

$$
V_{\pi} \simeq \mathcal{H}^{a n}(U) \otimes_{\mathcal{H}^{L, a n}\left(U_{\varpi}\right)} V_{t^{L}}
$$

(see Subsection 4.1), where $V_{t^{L}}$ denotes the representation space $V$ with $\mathcal{H}^{L}$ action defined by $h \rightarrow \delta\left(\phi_{t^{L}}(h)\right)$. Put $\pi^{\prime}=\pi\left(\mathcal{R}_{M}, W_{M} r_{M}^{\prime}, \delta^{\prime}, s^{M}\right)$.

Definition 4.32. For $t^{L} \in T^{L}$ such that $r_{L} t^{L}$ is $R_{L}$-generic, we define an intertwining isomorphism $A\left(n, \mathcal{R}_{L}, W_{L} r_{L}, \delta, t^{L}\right): V_{\pi} \rightarrow V_{\pi^{\prime}}$ by

$$
\begin{aligned}
A\left(n, \mathcal{R}_{L}, W_{L} r_{L}, \delta, t^{L}\right): \mathcal{H} \otimes_{\mathcal{H}^{L}} V_{t^{L}} & \rightarrow \mathcal{H} \otimes_{\mathcal{H}^{M}} V_{s^{M}}^{\prime} \\
h \otimes v & \rightarrow h \iota_{n^{-1}}^{0} \otimes \tilde{\delta}(v)
\end{aligned}
$$

It is easy to check that this is well defined and that this map intertwines the $\mathcal{H}$ actions.

Theorem 4.33. Recall the compact realization $V_{\pi}=\mathcal{H}\left(W^{L}\right) \otimes V$, with its inner product $\langle\cdot, \cdot\rangle_{\pi}$ (see Proposition 4.19).

(i) In the "compact realization", the intertwining map

$$
A\left(n, \mathcal{R}_{L}, W_{L} r_{L}, \delta, t^{L}\right): \mathcal{H}\left(W^{L}\right) \otimes V \rightarrow \mathcal{H}\left(W^{M}\right) \otimes V^{\prime}
$$

is rational as a function of induction parameter $t^{L}$, and regular outside the set of zeroes of the functions $t^{L} \rightarrow \Delta_{\alpha} c_{\alpha}\left(u\left(r_{L}\right) t^{L}\right)$, where $\alpha$ runs over the set of positive roots in $R_{1}$ such that $n(\alpha)<0$, and $u\left(r_{L}\right)$ (with $u \in W_{L}$ ) runs over the set of $X_{L^{-}}$ weights in $V$.

(ii) When $t^{L} \in T_{u}^{L}$ and $A\left(n, \mathcal{R}_{L}, W_{L} r_{L}, \delta, t^{L}\right)$ is regular at $t^{L}$, then in fact $A\left(n, \mathcal{R}_{L}, W_{L} r_{L}, \delta, t^{L}\right)$ is unitary with respect to the inner products $\langle\cdot, \cdot\rangle_{\pi}$ and $\langle\cdot, \cdot\rangle_{\pi^{\prime}}$.

(iii) With respect to these inner products we have

$$
A^{*}\left(n, \mathcal{R}_{L}, W_{L} r_{L}, \delta, t^{L}\right)=A\left(n^{-1}, \mathcal{R}_{M}, W_{M} r_{M}^{\prime}, \Psi_{n}(\delta), n\left(t^{L}\right)\right) .
$$


ON THE SPECTRAL DECOMPOSITION OF AFFINE HECKE ALGEBRAS 81

Proof. (i) The representation $\pi$ is cyclic and generated by $1 \otimes v$, with $v \neq 0$ an arbitrary vector in $V$. By the intertwining property it is therefore enough to show that $A\left(n, \mathcal{R}_{L}, W_{L} r_{L}, \delta, t^{L}\right)(1 \otimes v) \in \mathcal{H}\left(W^{L}\right) \otimes$ $V$ is meromorphic in $t^{L}$, and regular outside the indicated set. Using equation (4.6), we have

$$
A\left(n, \mathcal{R}_{L}, W_{L} r_{L}, \delta, t^{L}\right)(1 \otimes v)=\pi\left(\iota_{n^{-1}}\right) \pi\left(\prod_{\alpha>0, n(\alpha)<0} \Delta_{\alpha} c_{\alpha}\right)^{-1}(1 \otimes \tilde{\delta}(v)) .
$$

Since $\pi(h)$ is a regular function on $T^{L}$ for all $h \in \mathcal{H}$, this is a rational expression. The generalized $X$-weights in $1 \otimes V_{t^{L}}$ are of the form $u\left(r_{L}\right) t^{L}$. So the inverse of $\pi\left(\prod_{\alpha>0, n(\alpha)<0} \Delta_{\alpha} c_{\alpha}\right)$ can have poles only at the indicated set.

(ii) In order to see the unitarity, we first note that by Corollary 4.29(ii) and Theorem 4.30, the statement is equivalent to the unitarity with respect to the inner products on $V_{\pi}$ and $V_{\pi^{\prime}}$ defined by the embedding of these spaces in $\overline{\mathcal{H}^{t}}$ as in Corollary 4.29. Choose an embedding $\bar{i}: V \rightarrow \mathcal{H}_{L}^{r_{L}}$ as in Corollary 4.29. By Theorem 4.30 and Corollary 4.26 , the map $\left.\Delta_{n \varpi, n \varpi}\right|_{\bar{j}\left(V_{\pi, \varpi}\right)}$ is an isometry. By equation (4.48) we see that this isometry satisfies, for $h \in H^{L}, \psi_{n}(h) \cdot \Delta_{n \varpi, n \varpi}(\bar{j}(1 \otimes v))=$ $\Delta_{n \varpi, n \varpi}\left(\bar{j}\left(1 \otimes \delta_{t^{L}}(h)(v)\right)\right)$. Hence if we identify $V_{t^{L}}$ with $\bar{j}\left(V_{\pi, \varpi}\right)$, we can define $V_{n\left(t^{L}\right)}^{\prime}=\Delta_{n \varpi, n \varpi}\left(V_{t^{L}}\right)$. Then the map $\tilde{\delta}=\Delta_{n \varpi, n \varpi}$ defines a unitary map satisfying (4.69).

Now it is clear, in the notation of Corollary 4.29, that we can identify the space $\overline{j^{\prime}}\left(V_{\pi^{\prime}, \varpi^{\prime}}\right)$ with $\bar{j}\left(V_{\pi, \varpi}\right) \iota_{n^{-1}}^{0}$, and the map $A\left(n, \mathcal{R}_{L}, W_{L} r_{L}, \delta, t^{L}\right)$ is then identified with the right multiplication with $\iota_{n^{-1}}^{0}$, thus with $\Delta_{\varpi, n \varpi}$. This is unitary on $\bar{j}\left(V_{\pi, \varpi}\right)$, by Corollary 4.31 . By the irreducibility of $V_{\pi}$ and $V_{\pi^{\prime}}$ this concludes the proof of (ii).

(iii) This last assertion of the Theorem is now obvious, since these maps are clearly inverse to each other.

The next Corollary is an important classical application of the unitarity of the intertwiners, see [4], Théorème 2.

Corollary 4.34. The intertwining map $t^{L} \rightarrow A\left(n, \mathcal{R}_{L}, W_{L} r_{L}, \delta, t^{L}\right)$ extends holomorphically to an open neighborhood of $T_{u}^{L}$ in $T^{L}$.

Proof. By the unitarity on $T_{u}^{L}$, the meromorphic matrix entries of $A\left(n, \mathcal{R}_{L}, W_{L} r_{L}, \delta, t^{L}\right)$ are uniformly bounded for $t^{L} \in T_{u}^{L}$ in the open set of $T_{u}^{L}$ where $A\left(n, \mathcal{R}_{L}, W_{L} r_{L}, \delta, t^{L}\right)$ is well defined and regular. This is the complement of the collection of real codimension 1 cosets in $T_{u}^{L}$ as described in Theorem 4.33. This implies that the singularities of the matrix entries which meet $T_{u}^{L}$ are actually removable. 


\subsection{The Plancherel decomposition of the trace $\tau$}

In this section we rewrite the decomposition Theorem 4.25 as a decomposition of $\tau$ in terms of characters of irreducible tempered representations induced from cuspidal representations of the subalgebras $\mathcal{H}^{P}$.

Using the results of the previous section, we show that the corresponding Fourier homomorphism maps $\mathcal{H}$ into a certain space of smooth sections defined over orbits of irreducible cuspidal representations of the subalgebras $\mathcal{H}^{P}$, equivariant with respect to the natural actions of intertwining operators.

This final formulation of the results (Theorem 4.43) is inspired by and parallel to the notations used in the theory of the Harish-Chandra Plancherel formula for p-adic groups, as treated in [49] and [12].

We need to develop some notations. Let $P \subset F_{0}$ denote the power set of $F_{0}$, and let $\Gamma$ denote the set of all pairs $\gamma=\left(\mathcal{R}_{P}, W_{P} r\right)$ with $P \in \mathcal{P}, \mathcal{R}_{P}$ the associated parabolic root datum, and $W_{P} r$ an orbit of residual points in $T_{P}$. We consider the disjoint union of the set of all triples $\Lambda=\left\{\left(\mathcal{R}_{P}, W_{P} r, t\right)\right\}$, where $\left(\mathcal{R}_{P}, W_{P} r\right) \in \Gamma$ and $t \in T_{u}^{P}$. Let $\Lambda_{\mathcal{R}_{P}, W_{P} r}=\Lambda_{\gamma}$ be the subspace of such triples with $\gamma=\left(\mathcal{R}_{P}, W_{P} r\right) \in \Gamma$ fixed. Hence for all $\gamma \in \Gamma, \Lambda_{\gamma}$ is a copy of $T_{u}^{P}$ and $\Lambda=\cup \Lambda_{\gamma}$ (disjoint union). Therefore $\Lambda$ is a disjoint union of finitely many compact tori, which gives $\Lambda$ the structure of a compact Hausdorff space. In addition, each $\Lambda_{\gamma}$ comes with its (normalized) Haar measure, thus defining a measure on $\Lambda$. We denote by $\Lambda_{\gamma}^{\text {gen }}$ the open, dense subset of triples $\left(\mathcal{R}_{P}, W_{P} r, t\right)$ such that $\left(\mathcal{R}_{P}, W_{P} r\right)=\gamma$ and $r t$ is $R_{P \text {-generic. We put }}$ $\Lambda^{\text {gen }}=\cup \Lambda_{\gamma}^{\text {gen }}($ disjoint union over $\gamma \in \Gamma$ ).

Define a map $m: \Lambda \rightarrow S \subset W_{0} \backslash T$ by

$$
m\left(\mathcal{R}_{P}, W_{P} r, t\right)=W_{0}(r t)
$$

By Theorem 3.29, $m$ is surjective, and obviously $m$ is continuous and finite.

Let $P, Q \in \mathcal{P}$. Recall the set $W(P, Q) \subset W_{0}$ defined by $W(P, Q):=$ $\left\{n \in W_{0} \mid n(P)=Q\right\}$. We put $W(P)=W(P, P)$, which is a subgroup of $W_{0}$ and acts on $R_{P}$ through diagram automorphisms. Observe that $W(P) \subset N_{W_{0}}\left(W_{P}\right)$ is a subgroup which is complementary to the normal subgroup $W_{P} \subset N_{W_{0}}\left(W_{P}\right)$. Moreover, $W(P, Q)$ is a left $W(P)$ coset and a right $W(Q)$ coset.

The action of $n \in W(P, Q)$ on $T$ restricts to isomorphisms $T_{P} \rightarrow T_{Q}$ and $T^{P} \rightarrow T^{Q}$. Recall that $K_{P}=T_{P} \cap T^{P}$, so that $n \in W(P, Q)$ gives rise to an isomorphism $n: K_{P} \rightarrow K_{Q}$. 
Consider the groupoid $\mathcal{W}$ whose set of objects is $\mathcal{P}$, with morphisms $\operatorname{Hom}_{\mathcal{W}}(P, Q)=\mathcal{W}(P, Q):=K_{Q} \times W(P, Q)$ and the composition defined by $\left(k_{1} \times n_{1}\right) \circ\left(k_{2} \times n_{2}\right)=k_{1} n_{1}\left(k_{2}\right) \times\left(n_{1} \circ n_{2}\right)$. We denote by $\mathcal{W}(P)$ the group $\mathcal{W}(P)=\mathcal{W}(P, P)$.

If $k \times n \in \mathcal{W}(P, Q)$ we define for $\gamma=\left(\mathcal{R}_{P}, W_{P} r\right) \in \Gamma_{P}$ :

$$
(k \times n)(\gamma)=(k \times n)\left(\mathcal{R}_{P}, W_{P} r\right):=\left(\mathcal{R}_{Q}, W_{Q}\left(k^{-1} n(r)\right)\right) .
$$

This defines a left action of $\mathcal{W}$ on $\Gamma$. If $t \in T_{u}^{P}$ then $(\gamma, t) \in \Lambda_{\gamma}$, and we define

$$
(k \times n)(\gamma, t):=((k \times n)(\gamma), k n(t)) .
$$

This defines a left action of $\mathcal{W}$ on $\Lambda$. With these definitions we obviously have

$$
m(g(\lambda))=m(\lambda)
$$

for all $g \in \operatorname{Hom}(\mathcal{W})$ and $\lambda \in \Lambda$ such that $g(\lambda)$ is defined. In other words, $m$ is $\mathcal{W}$-invariant.

Lemma 4.35. The action of $\mathcal{W}$ on $\Lambda^{\text {gen }}$ is free.

Proof. Let $\lambda=\left(\mathcal{R}_{P}, W_{P} r, t\right) \in \Lambda_{\mathcal{R}_{P}, W_{P} r}^{\text {gen }}$ and let $g=k \times n \in \mathcal{W}(P, Q)$ be such that $g(\lambda)=\lambda$. Then $Q=P, g$ fixes $W_{P} r$, and $k n(t)=t$. We have $g\left(W_{P} r\right):=W_{P}\left(k^{-1} n(r)\right)$, thus $n(r)=k w(r)$ for some $w \in W_{P}$. Hence $n(r t)=n(r) n(t)=w(r)(k n(t))=w(r t)$. Since $r t$ is $R_{P}$-generic this implies that $w^{-1} n \in W_{P}$. Hence $n=e$, and thus also $k=e$.

Lemma 4.36. Let $L$ be a residual subspace, and let $t=r_{L} t^{L} \in L^{\text {temp }}$ be $R_{L}$ generic. Then $m^{-1}\left(W_{0} t\right)$ is a $\mathcal{W}$-orbit in $\Lambda$.

Proof. By making a suitable choice of $t$ in the orbit $W_{0} t$ we may assume that $R_{L}=R_{P}$ for some $P \in \mathcal{P}$. We write $r_{P}$ instead of $r_{L}$ and $t^{P}$ instead of $t^{L}$. Thus it is assumed that $t=r_{P} t^{P} \in L^{t e m p}=r_{P} T_{u}^{P}$ is $R_{P}$-generic. Define $\lambda:=\left(\mathcal{R}_{P}, W_{P} r_{P}, t^{P}\right) \in \Lambda_{\mathcal{R}_{P}, W_{P} r_{P}}^{\text {gen }}$. Clearly, $\mathcal{W} \cdot \lambda \subset$ $m^{-1}\left(W_{0} t\right)$ by the $\mathcal{W}$-invariance of $m$.

Conversely, suppose that $\mu=\left(\mathcal{R}_{Q}, W_{Q} r_{Q}, t^{Q}\right) \in m^{-1}\left(W_{0} t\right)$. Hence there exists a $w \in W_{0}$ such that $r_{Q} t^{Q}=w t=w\left(r_{P}\right) w\left(t^{P}\right)$. This is an element of the tempered residual subspace $L_{Q}^{\text {temp }}:=r_{Q} T_{u}^{Q}$, so that $R_{Q} \subset R_{P(w t)}$. Since $t \in L^{t e m p}$ is $R_{P}$ generic, we have $R_{P(t)}=$ $R_{P}$ by Corollary 4.13. Because $R_{P(w t)}=w\left(R_{P(t)}\right)$, we obtain $R_{Q} \subset$ $w\left(R_{P}\right)$. This implies that $w\left(L^{t e m p}\right)=w(t) w\left(T_{u}^{P}\right) \supset r_{Q} T_{u}^{Q}=L_{Q}^{t e m p}$. By Theorem 7.17 we see that $w\left(L^{t e m p}\right)=L_{Q}^{t e m p}$. Hence we have $w\left(R_{P}\right)=$ $R_{Q}, w\left(T_{P}\right)=T_{Q}$ and $w\left(T^{P}\right)=T^{Q}$. We conclude that $r_{Q}^{-1} w\left(r_{P}\right)=k \in$ $K_{Q}$. There exists a unique $u \in W_{Q}$ such that $u w \in W(P, Q)$. One easily checks that $\mu=(k \times u w)(\lambda)$. 
Note that it follows that the intersection $m^{-1}\left(W_{0} t\right) \cap \Lambda_{\mathcal{R}_{P}, W_{P} r_{P}}$ is contained in $\Lambda_{\mathcal{R}_{P}, W_{P} r_{P}}^{\text {gen }}$ (for any choice of $P \in \mathcal{P}$ and $W_{P} r_{P}$ ).

Corollary 4.37. We form the quotients $\Sigma=\mathcal{W} \backslash \Lambda$ and $\Sigma^{\text {gen }}=\mathcal{W} \backslash \Lambda^{\text {gen }}$. The map $m$ factors through $\Sigma$, and defines a homeomorphism (also denoted by $m$ ) from $\Sigma^{\text {gen }}$ onto the open dense set $S^{g e n}:=m\left(\Lambda^{\text {gen }}\right) \subset S$.

Proof. By equation (4.78), $m$ is well defined on $\Sigma$, and thus $m(\Sigma)=$ $m(\Lambda)=S$. By the previous lemma, the set $\Lambda^{g e n}$ is $m$-saturated. Since $m$ is closed, this implies that $S^{g e n}=m\left(\Lambda^{g e n}\right) \subset S$ is open (and obviously dense) in $S=m(\Sigma)$. Finally, again by the previous lemma, $m$ is injective on $\Sigma^{g e n}$. Thus, being a closed map, $m$ is homeomorphic onto its image.

$\Sigma$ can be realized as a disjoint union of orbifolds as follows. Choose a complete set $\Gamma_{a}$ of representatives for the association classes (the orbits of $\mathcal{W}$ ) of elements in $\Gamma$. Put

$$
\Sigma_{\gamma}:=\mathcal{W}(\gamma) \backslash \Lambda_{\gamma}
$$

where $\mathcal{W}(\gamma)$ denotes the isotropy group of $\gamma \in \Gamma$ in $\mathcal{W}$. Then

$$
\Sigma \simeq \cup \Sigma_{\gamma}
$$

where the (disjoint) union is taken over the set of $\gamma \in \Gamma_{a}$.

4.5.1. Groupoid $\mathcal{W}_{\Xi}$ of standard induction data. Recall the complete set of representatives $\Delta_{\gamma}\left(\gamma=\left(\mathcal{R}_{P}, W_{P} r_{P}\right) \in \Gamma\right)$ of the irreducible discrete series representations with central character $W_{P} r_{P}$ of $\mathcal{H}_{P}$. We denote by $\Delta=\cup \Delta_{\gamma}$ the disjoint union of these sets over all $\gamma \in \Gamma$. The composition $\Delta \rightarrow \Gamma \rightarrow \mathcal{P}$ gives a surjection of $\Delta \rightarrow \mathcal{P}$, whose fibers are denoted by $\Delta_{P}$.

There is a natural left action $\Psi$ of $\mathcal{W}$ on $\Delta$ as follows: When $k \in$ $K_{P}=T^{P} \cap T_{P}$, we have an automorphism $\psi_{k}: \mathcal{H}_{P} \rightarrow \mathcal{H}_{P}$ defined by $\psi_{k}\left(\theta_{x} T_{w}\right)=k(x) \theta_{x} T_{w}$. This induces an isomorphism $\psi_{k}: \overline{\mathcal{H}_{P}^{r_{P}}} \rightarrow$ $\overline{\mathcal{H}_{P}^{k^{-1} r_{P}}}$. We define a bijection $\Psi_{k}$ from $\Delta_{\mathcal{R}_{P}, W_{P} r_{P}}$ to $\Delta_{\mathcal{R}_{P}, k^{-1} W_{P} r_{P}}$ by $\Psi_{k}(\delta) \simeq \delta \circ \psi_{k}^{-1}$.

Let $Q \in F_{0}$ be associate to $P$, and $n \in W(P, Q)$. Then $n$ induces an isomorphism of root data and labels $\left(\mathcal{R}_{P}, q\right) \rightarrow\left(\mathcal{R}_{Q}, q\right)$, thus inducing an isomorphism $\psi_{n}$ on $\mathcal{H}_{P} \rightarrow \mathcal{H}_{Q}$. Recall that $\psi_{n}$ induces an isomorphism $\psi_{n}: \overline{\mathcal{H}_{P}^{r_{P}}} \rightarrow \overline{\mathcal{H}_{Q}^{n\left(r_{P}\right)}}$ (Corollary 4.26), and thus a bijection $\Psi_{n}: \Delta_{\mathcal{R}_{P}, W_{P} r_{P}} \rightarrow \Delta_{\mathcal{R}_{Q}, W_{Q} n\left(r_{P}\right)}$ by $\Psi_{n}(\delta) \simeq \delta \circ \psi_{n}^{-1}$. One easily checks that these definitions combine to define a left action $\Psi$ of $\mathcal{W}$ on $\Delta$, compatible with the surjection $\Delta \rightarrow \mathcal{P}$ mentioned above. 
Consider the product $\Xi:=\Lambda \times_{\Gamma} \Delta$. This set comes equipped with a natural surjection $\Xi \rightarrow \mathcal{P}$ and compatible left action of $\mathcal{W}$ (the diagonal action). We form the cross product $\mathcal{W}_{\Xi}:=\mathcal{W} \times_{\mathcal{P}} \Xi$, which has itself a natural groupoid structure with $\operatorname{Obj}\left(\mathcal{W}_{\Xi}\right):=\Xi$, and $\operatorname{Hom}_{\mathcal{W}_{\Xi}}\left(\xi_{1}, \xi_{2}\right):=$ $\left\{w \in \mathcal{W} \mid w\left(\xi_{1}\right)=\xi_{2}\right\}$. The composition maps are defined by the composition in $\mathcal{W}$. We will refer to this structure as the groupoid of standard induction data of $\mathcal{H}$. Its set of objects $\Xi$ has the structure of a disjoint union of compact tori, and with this structure $\mathcal{W}_{\Xi}$ is obviously a smooth compact groupoid.

Recall that we associated to each $\xi=\lambda \times \delta=\left(\mathcal{R}_{P}, W_{P} r_{P}, t\right) \times$ $\left(\mathcal{R}_{P}, \delta\right) \in \Xi$ (i.e. $\delta$ is an irreducible discrete series representation of $\mathcal{H}\left(\mathcal{R}_{P}, q\right)$ with central character equal to $\left.W_{P} r_{P}\right)$ a tempered, unitary representation $\pi(\xi)=\pi\left(\mathcal{R}_{P}, W_{P} r_{P}, \delta, t\right)$ of $\mathcal{H}$ with central character $m(\lambda)=W_{0}\left(r_{P} t\right)$ and representation space $V_{\pi(\xi)}=\mathcal{H}\left(W^{P}\right) \otimes V_{\delta}$ (the compact realization) (cf. Definition 4.17, Proposition 4.19 and Proposition 4.20).

For every $(g, \xi) \in \mathcal{W}_{\Xi}$ with source $\xi=\lambda \times \delta$, we choose a unitary isomorphism $\tilde{\delta}_{g}: V_{\delta} \rightarrow V_{\Psi_{g}(\delta)}$ so that we have

$$
\Psi_{g}(\delta)(h) \circ \tilde{\delta}_{g}=\tilde{\delta}_{g} \circ \delta\left(\psi_{g}^{-1} h\right)
$$

for $h \in \mathcal{H}_{P}$ (where $\left.P=P(\delta)\right)$.

This enables us to define intertwining operators (depending on the choices of the isomorphisms $\tilde{\delta}_{g}$ )

$$
A(g, \xi) \in \operatorname{Hom}_{\mathcal{H}}\left(V_{\pi(\xi)}, V_{\pi(g(\xi))}\right)
$$

as follows:

For $k \in K_{P}$ and $h \in \mathcal{H}^{P}$ we have $\phi_{k t}(h)=\psi_{k}\left(\phi_{t}(h)\right)$, so that if $h \in \mathcal{H}^{P}$ we have that $\tilde{\delta}_{k} \circ \delta\left(\phi_{t}(h)\right)=\Psi_{k}(\delta)\left(\phi_{k t}(h)\right) \circ \tilde{\delta}_{k}$. With this notation we have for each $\delta \in \Delta_{\mathcal{R}_{P}, W_{P} r_{P}}$, in view of Proposition 4.19, a unitary intertwining isomorphism

$$
\operatorname{Id} \otimes \tilde{\delta}_{k}: \pi(\xi) \rightarrow \pi(k(\xi)) .
$$

We denote this unitary intertwining operator by $A\left(k, \mathcal{R}_{P}, W_{P} r, \delta, t\right)$ or more simply $A(k, \xi)$. Notice that it is constant, i.e. independent of $t$.

For $n \in W(P, Q)$ (with $P, Q \in \mathcal{P}$ associate subsets) we defined (cf. Theorem 4.33 and Corollary 4.34) an intertwining isomorphism (depending on the choice of $\tilde{\delta}_{n}$ )

$A\left(n, \mathcal{R}_{P}, W_{P} r_{P}, \delta, t\right): \pi\left(\mathcal{R}_{P}, W_{P} r_{P}, \delta, t\right) \rightarrow \pi\left(\mathcal{R}_{Q}, W_{Q} n\left(r_{P}\right), \Psi_{n}(\delta), n(t)\right)$,

which is rational in $t$, well defined and regular in a neighborhood of $T_{u}^{P}$, and unitary for $t \in T_{u}^{P}$. We now denote this isomorphism by $A(n, \xi)$. 
The above intertwining isomorphisms combine (as one easily checks directly from the definitions) to a functor

$$
\mathcal{W}_{\Xi} \rightarrow P R e p_{\text {unit temp }}(\mathcal{H})
$$

where $P \operatorname{Rep} p_{\text {unit,temp }}(\mathcal{H})$ denotes the category of finite dimensional, tempered, unitary modules over $\mathcal{H}$, with morphisms $\operatorname{Hom}_{P R e p}\left(\pi_{1}, \pi_{2}\right)=$ $P U_{\mathcal{H}}\left(V_{\pi_{1}}, V_{\pi_{2}}\right)$ (the space of unitary intertwiners modulo the action of scalars).

Summarizing the above we have:

Theorem 4.38. There exists an induction functor

$$
\pi: \mathcal{W}_{\Xi} \rightarrow \operatorname{PRep}_{\text {unit temp }}(\mathcal{H})
$$

such that for $\xi=\left(\mathcal{R}_{P}, W_{P} r_{P}, t\right) \times\left(\mathcal{R}_{P}, \delta\right) \in \Xi$ and $(g, \xi) \in \mathcal{W}_{\Xi}$ (thus $g \in \mathcal{W}$ with source $P(\xi)=P$ ), $\pi(\xi):=\pi\left(\mathcal{R}_{P}, W_{P} r_{P}, \delta, t\right)$ and $\pi(g, \xi):=A(g, \xi)=A\left(n, \mathcal{R}_{P}, W_{P} r_{P}, \delta, t\right)$.

4.5.2. Generic spectrum. Consider the natural projection

$$
p_{\Sigma}: \mathcal{W}_{\Xi} \backslash \Xi=\mathcal{W} \backslash\left(\Lambda \times_{\Gamma} \Delta\right) \rightarrow \Sigma=\mathcal{W} \backslash \Lambda
$$

Since the action of $\mathcal{W}$ is free on the set of generic points $\Lambda^{\text {gen }}$, we obtain a finite covering

$$
p_{\Sigma}: \mathcal{W}_{\Xi} \backslash \Xi^{g e n} \rightarrow \Sigma^{g e n},
$$

where $\Xi^{\text {gen }}:=\Lambda^{\text {gen }} \times_{\Gamma} \Delta$.

By what was said in the previous subsection and Corollary 4.18, it is clear that the map (see Proposition 2.13 for the definition of $p_{z}$ ):

$$
\begin{aligned}
{[\pi]: \Xi^{g e n} } & \rightarrow p_{z}^{-1}\left(S^{g e n}\right) \\
\xi & \rightarrow[\pi(\xi)]
\end{aligned}
$$

factors through the quotient $\mathcal{W}_{\Xi} \backslash \Xi^{\text {gen }}$. We thus have the following commutative diagram:

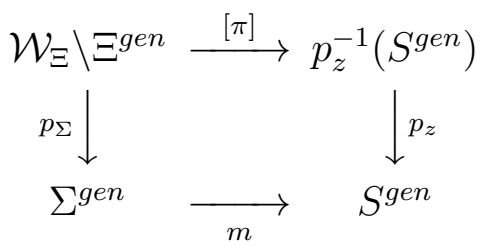

Theorem 4.39. The map $[\pi]$ in the diagram 4.90 is a homeomorphism.

Proof. The topology on $\hat{\mathfrak{C}}$ is second countable since $\mathfrak{C}$ is separable. Thus, in order to check the continuity of $[\pi]$, it suffices to check that $[\pi]$ maps a converging sequence $\lambda_{i} \times \delta \rightarrow \lambda \times \delta \in \Lambda_{\mathcal{R}_{P}, W_{P} r}$ to a converging sequence in $\hat{\mathfrak{C}}$. We check this using the Fell-topology description of 
the topology of $\hat{\mathfrak{C}}$ (see [16]). By [16], Proposition 1.17, restriction to the dense subalgebra $\mathcal{H} \subset \mathfrak{C}$ is a homeomorphism with respect to the Fell topologies. Let $V_{\lambda \times \delta}=\mathcal{H}\left(W^{P}\right) \otimes V$ be the representation space of $\pi(\lambda \times \delta)$ (with $\left.\lambda \in \Lambda_{\mathcal{R}_{P}, W_{P} r}\right)$. We equip $V_{\lambda \times \delta}$ with the inner product $\langle\cdot, \cdot\rangle$ of Proposition 4.19 (which is independent of $\lambda \in \Lambda_{\mathcal{R}_{P}, W_{P} r}$ ), and we choose an orthonormal basis $\left(e_{i}\right)$ of $V_{\lambda \times \delta}$. In order to check that $\pi\left(\lambda_{i} \times \delta\right) \rightarrow \pi(\lambda \times \delta)$ in the Fell topology with respect to $\mathcal{H}$, we need to check that for all $h \in \mathcal{H}, \pi\left(\lambda_{i} \times \delta\right)(h)_{k, l} \rightarrow \pi(\lambda \times \delta)(h)_{k, l}$ for all matrix coefficients. This is clear since the matrix coefficients are regular functions of the induction parameter.

To see that the map $[\pi]$ is closed, assume that we have a sequence $\rho_{i}=[\pi]\left(\lambda_{i} \times \delta_{i}\right)$ converging to $\rho \in p_{z}^{-1}\left(S^{g e n}\right)$. Since $m$ is a homeomorphism and $\Sigma^{g e n}$ is a finite quotient of $\Lambda^{\text {gen }}$, we may assume that $\lambda_{i}$ converges, to $\lambda_{0} \in \Lambda^{\text {gen }}$ say, by possibly replacing the sequence by a subsequence. Since $\Delta_{\mathcal{R}_{P}, W_{P} r}$ is finite for each $R_{P}$ and $W_{P} r$, we may assume that $\forall i: \delta_{i}=\delta$, again by taking a subsequence. Then $d=\operatorname{dim}\left(\rho_{i}\right)$ is independent of $i$, and lower semi-continuity of $\operatorname{dim}$ on $\hat{\mathfrak{C}}$ implies that $\operatorname{dim}(\rho) \leq d$. Choose an orthonormal basis $B$ for $\rho$. Convergence in the Fell-topology means that there exists for all $i$ an orthonormal subset $B_{i}$ of size $\operatorname{dim}(\rho)$ in the representation space $V_{\rho_{i}}=\mathcal{H}\left(W^{P}\right) \otimes V$ of $\rho_{i}$, such that the matrix coefficients of $\rho_{i}$ with respect to $B_{i}$ converge to the matrix coefficients of $\rho$ with respect to $B$. By the independence of the inner product $\langle\cdot, \cdot\rangle$ of the induction parameter (Proposition 4.19) we may assume, by further restricting to a subsequence, that the sets $B_{i}$ converge in $\mathcal{H}\left(W^{P}\right) \otimes V$ to an orthonormal set $B_{0}$. It follows that the matrix of $\rho(x)$ with respect to $B$ equals a principal block of the matrix of $\pi\left(\lambda_{0} \times \delta\right)(x)$ with respect to a suitable orthonormal basis $\tilde{B}$ of $\mathcal{H}\left(W^{P}\right) \otimes V$ for $\pi\left(\lambda_{0} \times \delta\right)$. Since $\pi\left(\lambda_{0} \times \delta\right)$ is irreducible it is easy to see that this is impossible unless $\rho \simeq \pi\left(\lambda_{0} \times \delta\right)$.

The map $[\pi]$ is injective by Corollary 4.18 and Lemma 4.36 .

Finally, by Theorem 3.25, Theorem 3.29, Theorem 4.23 and Theorem 4.25 we see that the complement of $[\pi]\left(\mathcal{W}_{\Xi} \backslash \Xi^{r e g}\right)$ has measure 0 in $\hat{\mathfrak{C}}^{*}$ with respect to the Plancherel measure of the representation $\mathfrak{H}$ of $\mathfrak{C}$. The support of the Plancherel measure is equal to $\hat{\mathfrak{C}}$, since $\mathfrak{H}$ is a faithful representation of $\mathfrak{C}$ (by definition of $\mathfrak{C}$ ). Thus the closure of $[\pi]\left(\mathcal{W}_{\Xi} \backslash \Xi^{r e g}\right)$ is $\hat{\mathfrak{C}}$. But $[\pi]\left(\mathcal{W}_{\Xi} \backslash \Xi^{r e g}\right) \subset p_{z}^{-1}\left(S^{r e g}\right)$ is closed as we have seen above, implying that $[\pi]$ is surjective.

Corollary 4.40. The restriction of the map $p_{z}$ of Corollary 2.13 to $p_{z}^{-1}\left(S^{r e g}\right)$ is a covering map. 
4.5.3. Fourier transform. Let $\tilde{\mathcal{O}} \subset \Xi$ be a connected component of $\Xi$. Thus there exists a $\delta \in \Delta$ such that $\tilde{\mathcal{O}}=\Lambda_{\gamma} \times\{\delta\}:=\tilde{\mathcal{O}}_{\delta}$, where $\gamma=\gamma(\delta) \in \Gamma$. Explicitly, if $\gamma(\delta)=\left(\mathcal{R}_{P}, W_{P} r_{P}\right)$ then $\tilde{\mathcal{O}}_{\delta}$ is a copy of the subtorus $T_{u}^{P} \subset T_{u}$.

The representation space $V_{\pi(\xi)}$ of $\pi(\xi)$ is equal to $V_{\pi(\xi)}=\mathcal{H}\left(W^{P}\right) \otimes V_{\delta}$ for $\xi \in \tilde{\mathcal{O}}_{\delta}$ with $\delta \in \Delta_{P}$. In particular, $V_{\pi(\xi)}$ depends only on the connected component $\tilde{\mathcal{O}}_{\delta}$ of $\Xi$ containing $\xi$, and not on the choice of $\xi \in \tilde{\mathcal{O}}_{\delta}$. We will use the notation $i\left(V_{\delta}\right):=\mathcal{H}\left(W^{P}\right) \otimes V_{\delta}=V_{\pi(\xi)}$ for any choice of $\xi \in \tilde{\mathcal{O}}_{\delta}$ (where $P=P(\delta) \in \mathcal{P}$ ).

We form the trivial fiber bundle $\mathcal{V}_{\tilde{\mathcal{O}}}=\tilde{\mathcal{O}}_{\delta} \times i\left(V_{\delta}\right)$ over $\tilde{\mathcal{O}}=\tilde{\mathcal{O}}_{\delta}$, and put

$$
\mathcal{V}_{\Xi}:=\cup_{\delta \in \Delta} \mathcal{V}_{\tilde{\mathcal{O}}_{\delta}} .
$$

We identify the connected component $\tilde{\mathcal{O}}_{\delta}$ of $\Xi$ with the compact torus $T_{u}^{P}(P=P(\delta))$. This allows us to define the function spaces $\operatorname{Pol}(\Xi)$ (Laurent polynomials on $\Xi$ ) and $C^{\infty}(\Xi)$. We also introduce the space $\operatorname{Rat}^{r e g}(\Xi)=\oplus_{\delta \in \Delta} \operatorname{Rat}^{r e g}\left(\tilde{\mathcal{O}}_{d}\right)$, where $\operatorname{Rat}^{\text {reg }}\left(\tilde{\mathcal{O}}_{d}\right)$ denotes the space of restrictions to $T_{u}^{P}$ (which we identify with $\tilde{\mathcal{O}}_{d}$ ) of rational functions on $T^{P}$ which are regular in a open neighborhood of $T_{u}^{P}$. The corresponding spaces of (global) sections are denoted by $\operatorname{Pol}\left(\mathcal{V}_{\Xi}\right)=\operatorname{Pol}(\Xi) \otimes \mathcal{V}_{\Xi}$, $C^{\infty}\left(\mathcal{V}_{\Xi}\right)=C^{\infty}(\Xi) \otimes \mathcal{V}_{\Xi}$, and $\operatorname{Rat}^{r e g}\left(\mathcal{V}_{\Xi}\right)=\operatorname{Rat}^{r e g}(\Xi) \otimes \mathcal{V}_{\Xi}$ respectively.

Recall that $\pi(g, \xi) \in P U_{\mathcal{H}}\left(i\left(V_{\delta}\right), i\left(V_{g d}\right)\right)$ (with $\xi=\lambda \times \delta=\left(\mathcal{R}_{P}, W_{P} r, t\right)$ $\times \delta$ ) is rational and regular for $t \in T^{P}$ in a neighborhood of $T_{u}^{P}$ (Corollary 4.34). We define

$$
\begin{aligned}
\operatorname{Pol} & \left.\operatorname{End}\left(\mathcal{V}_{\Xi}\right)\right)^{\mathcal{W}} \\
& =\left\{f \in \operatorname{Pol}\left(\operatorname{End}\left(\mathcal{V}_{\Xi}\right)\right) \mid \forall(g, \xi) \in \mathcal{W}_{\Xi}: \pi(g, \xi) f(\xi)=f(g \xi) \pi(g, \xi)\right\} \\
& \simeq \bigoplus_{\tilde{\mathcal{O}}} \operatorname{Pol}\left(\mathcal{V}_{\tilde{\mathcal{O}}}\right)^{\mathcal{W}(\tilde{\mathcal{O}}, \tilde{\mathcal{O}})}
\end{aligned}
$$

where the direct sum runs over a complete set of representatives of connected components $\tilde{\mathcal{O}}$ for the action of $\mathcal{W}$, and $\mathcal{W}\left(\tilde{\mathcal{O}}_{1}, \tilde{\mathcal{O}}_{2}\right)$ denotes the set of $w \in \mathcal{W}$ such that $w\left(\tilde{\mathcal{O}}_{1}\right)=\tilde{\mathcal{O}}_{2}$. We define the space of $\mathcal{W}_{\Xi^{-}}$ equivariant sections in other spaces of sections of $\operatorname{End}\left(\mathcal{V}_{\Xi}\right)$ similarly.

Definition 4.41. The Fourier transform is the algebra homomorphism

$$
\begin{aligned}
\mathcal{F}_{\mathcal{H}}: \mathcal{H} & \rightarrow \operatorname{Pol}\left(\operatorname{End}\left(\mathcal{V}_{\Xi}\right)\right)^{\mathcal{W}} \\
h & \rightarrow\{\xi \rightarrow \pi(\xi)(h)\}
\end{aligned}
$$

We would like to replace $\Xi$ by the set of equivalence classes of cuspidal representations of the standard parabolic subalgebras $\mathcal{H}^{P}$. This can be done as follows. Consider the subgroupoid $\mathcal{K} \subset \mathcal{W}$ of $\mathcal{W}$, with set of 
ON THE SPECTRAL DECOMPOSITION OF AFFINE HECKE ALGEBRAS 89

objects $\mathcal{P}$, and $\mathcal{K}\left(P_{1}, P_{2}\right)=\emptyset$ if $P_{1} \neq P_{2}$, and $\mathcal{K}(P, P)=K_{P}$. This subgroupoid is normal in the sense that $g K_{P} g^{-1}=K_{Q}$ if $g \in \mathcal{W}(P, Q)$. The quotient groupoid $\mathcal{W} / \mathcal{K}=\mathcal{W} / \mathcal{K}$ has $\mathcal{P}$ as set of objects, and $\mathcal{W} / \mathcal{K}(P, Q)=W(P, Q)$.

Suppose that $\delta_{t} \simeq \delta_{s}^{\prime}$ with $\delta, \delta^{\prime} \in \Delta_{\mathcal{R}_{P}}$ and $s, t \in T_{u}^{P}$. Then $W_{P} r t=$ $W_{P} r^{\prime} s$, and thus $s=k t$ for some $k \in K_{P}$, and $\delta_{s}^{\prime}=\Psi_{k}(\delta)_{k t}$. Conversely, in view of the text above (4.83), $\xi \simeq k(\xi)$ for every $k \in K_{P}$ and $\xi \in \Xi_{P}$, viewed as representation of $\mathcal{H}^{P}$.

The connected components $\mathcal{O}$ of the quotient $\mathcal{K} \backslash \Xi$ are called "orbits of twists of cuspidal representations" of the parabolic subalgebras $\mathcal{H}^{P}$. Such a component can be viewed as the collection of mutually inequivalent representations of $\mathcal{H}^{P}$ of the form $\delta_{t}$. It is isomorphic to a smooth quotient $\mathcal{O} \simeq \mathcal{K}(\tilde{\mathcal{O}}, \tilde{\mathcal{O}}) \backslash \tilde{\mathcal{O}}$, a finite quotient of the subtorus $T_{u}^{P} \subset T_{u}$.

We have $\mathcal{W} \backslash \Xi=(\mathcal{K} \backslash \mathcal{W}) \backslash(\mathcal{K} \backslash \Xi)$. For $\mathcal{O}$ a connected component of $\mathcal{K} \backslash \Xi$, we choose a connected component $\Xi \supset \tilde{\mathcal{O}} \rightarrow \mathcal{O}$ covering $\mathcal{O}$. Let $\tilde{\mathcal{O}}=\Lambda_{\gamma} \times \delta$ and write $P(\gamma)=P$. The isotropy group $\{k \in$ $\left.K_{P} \mid k(\tilde{\mathcal{O}})=\tilde{\mathcal{O}}\right\}$ equals the isotropy group $K_{\delta}$. Notice that $K_{\delta}$ is independent of the choice of $\tilde{\mathcal{O}} \rightarrow \mathcal{O}$. We define the principal fiber bundle $\mathcal{V}_{\mathcal{O}}:=\tilde{\mathcal{O}} \times_{\mathcal{K}_{\delta}} i\left(V_{\delta}\right)$ over $\mathcal{O}$. This fiber bundle is not necessarily trivial. We put

$$
\begin{aligned}
\operatorname{Pol}\left(\operatorname{End}\left(\mathcal{V}_{\mathcal{O}}\right)\right) & =\left(\bigoplus_{\tilde{\mathcal{O}}: \tilde{\mathcal{O}} \rightarrow \mathcal{O}} \operatorname{Pol}\left(\operatorname{End}\left(\mathcal{V}_{\tilde{\mathcal{O}}}\right)\right)\right)^{K_{P}} \\
& \simeq \operatorname{Pol}\left(\operatorname{End}\left(\mathcal{V}_{\tilde{\mathcal{O}}}\right)\right)^{K_{\delta}}
\end{aligned}
$$

and

$$
\operatorname{Pol}\left(\operatorname{End}\left(\mathcal{V}_{(\mathcal{K} \backslash \Xi)}\right)\right)=\bigoplus_{\mathcal{O} \text { orbit }} \operatorname{Pol}\left(\operatorname{End}\left(\mathcal{V}_{\mathcal{O}}\right)\right)
$$

The quotient $\mathcal{W} / \mathcal{K}$ acts on $\mathcal{K} \backslash \Xi$ and thus also on the set of orbits. Given orbits $\mathcal{O}_{1}, \mathcal{O}_{2}$ with $P\left(\mathcal{O}_{i}\right):=P_{i}$ and $O_{i}=\Lambda_{\gamma_{i}} \times \delta_{i}$, we have $\mathcal{W} / \mathcal{K}\left(\mathcal{O}_{1}, \mathcal{O}_{2}\right)=\left\{n \in \mathcal{W} / \mathcal{K}\left(P_{1}, P_{2}\right) \mid n\left(\mathcal{O}_{1}\right)=\mathcal{O}_{2}\right\}=\{n \in$ $\left.W\left(P_{1}, P_{2}\right) \mid \exists k \in K_{P_{2}}: k \times n \in \mathcal{W}\left(\delta_{1}, \delta_{2}\right)\right\}$. We denote this set by $W\left(\mathcal{O}_{1}, \mathcal{O}_{2}\right)$. We also put $W(\mathcal{O}):=W(\mathcal{O}, \mathcal{O})$.

In this way we get

$$
\begin{aligned}
\operatorname{Pol}\left(\operatorname{End}\left(\mathcal{V}_{\Xi}\right)\right)^{\mathcal{W}} & =\operatorname{Pol}\left(\operatorname{End}\left(\mathcal{V}_{(\mathcal{K} \backslash \Xi)}\right)\right)^{\mathcal{K} \backslash \mathcal{W}} \\
& \simeq \bigoplus_{\mathcal{O}} \operatorname{Pol}\left(\operatorname{End}\left(\mathcal{V}_{\mathcal{O}}\right)\right)^{W(\mathcal{O})},
\end{aligned}
$$

where the direct sum runs over a complete set of representatives of orbits $\mathcal{O}$ modulo the action of $\mathcal{W} / \mathcal{K}$ (association classes of orbits). 
We use similar notations for spaces of sections with coefficients in other types of functions spaces (e.g. continuous, $C^{\infty}$, etc.) in $\operatorname{End}\left(\mathcal{V}_{\Xi}\right)$ and $\operatorname{End}\left(\mathcal{V}_{(\mathcal{K} \backslash \Xi)}\right)$.

4.5.4. Averaging projections. Consider a function space $F$ on $\Xi$ which is a module over $\operatorname{Rat}^{r e g}(\Xi)$. Due to the regularity of the intertwining operators (cf. Corollary 4.34), there exists a natural averaging projection $f \rightarrow \bar{f}$ from $F\left(\operatorname{End}\left(\mathcal{V}_{\Xi}\right)\right)$ (sections of $\operatorname{End}\left(\mathcal{V}_{\Xi}\right)$ with coefficients in $F$ ) to $F\left(\operatorname{End}\left(\mathcal{V}_{\Xi}\right)\right)^{\mathcal{W}}$. It is defined by $\left(\right.$ where $\left.\mathcal{W}_{\xi}=\left\{g \in \mathcal{W} \mid(g, \xi) \in \mathcal{W}_{\Xi}\right\}\right)$

$$
\bar{f}(\xi)=\left|W_{\xi}\right|^{-1} \sum_{g \in \mathcal{W}_{\xi}} \pi(g, \xi)^{-1} f(g(\xi)) \pi(g, \xi) .
$$

Notice that the function space $F=\operatorname{Pol}(\Xi)$ is too small; in general the average of $f \in \operatorname{Pol}\left(\operatorname{End}\left(\mathcal{V}_{\Xi}\right)\right)$ will be in $\operatorname{Rat}^{r e g}\left(\operatorname{End}\left(\mathcal{V}_{\Xi}\right)\right)^{\mathcal{W}}$.

There is a similar averaging procedure $f \rightarrow \bar{f}_{\mathcal{K}}$ which sends the space of sections $F\left(\operatorname{End}\left(\mathcal{V}_{\Xi}\right)\right)$ to $F\left(\operatorname{End}\left(\mathcal{V}_{(\mathcal{K} \backslash \Xi)}\right)\right.$ ) (in this case $F$ should be a module over $\mathbb{C})$.

4.5.5. Plancherel formula. We now define the Plancherel measure on $\mathcal{W} \backslash \Xi$. The following proposition says that the natural action of $\mathcal{W}_{\Xi}$ (via $\psi$ ) on the residual algebras is through isomorphisms of Hilbert algebras.

Proposition 4.42. Let $\delta \in \Delta_{\mathcal{R}_{P}, W_{P} r}$ and let $g=(k \times n) \in \mathcal{W}_{\mathcal{R}_{P}, W_{P} r}$. We have (in the notation of Theorem 4.23) $d_{\mathcal{R}_{P}, \Psi_{g}(\delta)}=d_{\mathcal{R}_{P}, \delta}$.

Proof. This is a simple extension of Corollary 4.26, with a similar proof.

Let $\tilde{\mathcal{O}}=\Lambda_{\gamma} \times \delta$ and let $\mathcal{O}=K_{\delta} \backslash \tilde{\mathcal{O}}$. If $P=P(\gamma)$ then $\mathcal{O}$ is a copy of the subquotient torus $\mathcal{K}_{\delta} \backslash T_{u}^{P}$. For $\omega \in \mathcal{O}$ we put $d^{\mathcal{O}} \omega$ for the normalized Haar measure on $\mathcal{O}$. Let $\gamma=\left(\mathcal{R}_{P}, W_{P} r\right)$ and let $\omega=$ $\left(\mathcal{R}_{P}, W_{P} r, \delta, K_{\delta} t^{P}\right)$ be $R_{P}$-generic. Let $L^{t e m p}=r T_{u}^{P}$ denote a residual subspace underlying $\tilde{\mathcal{O}}$. We define

$$
\begin{aligned}
d \mu_{P l}(\pi(\omega)) & =\left|W_{0}\left(r t^{P}\right)\right|\left|W^{P}\right|^{-1} d_{\mathcal{R}_{P}, \delta} d \nu_{L}\left(r t^{P}\right) \\
& =\frac{\left|W_{P}\right|}{\left|W_{P} \cap W_{r}\right|} \nu_{\mathcal{R}_{P}}(\{r\}) d_{\mathcal{R}_{P}, \delta} k_{P} m^{P}\left(r t^{P}\right) d^{P}\left(t^{P}\right) \\
& =\mu_{\mathcal{R}_{P}, P l}(\{\delta\})\left|K_{P} \delta\right| m^{P}(\omega) d^{\mathcal{O}} \omega
\end{aligned}
$$

where $d_{\mathcal{R}_{P}, \delta}>0$ is the residual degree of $\delta$ in the residual algebra $\overline{\mathcal{H}_{L}^{r}}, \mu_{\mathcal{R}_{P}, P l}$ is given in Corollary 3.32, $m^{P}(\omega)=m^{L}(\omega)$ is the common value of $m^{L}$ (as defined in Proposition 3.27) on the $K_{\delta}$ orbit $\omega$, and 
ON THE SPECTRAL DECOMPOSITION OF AFFINE HECKE ALGEBRAS 91

$k_{P}:=\left|K_{P}\right|$. We have used that the isotropy subgroup $W_{r t^{P}}$ equals $W_{P} \cap W_{r}$ if $r t^{P}$ is $R_{P^{-} \text {generic. }}$

Recall that by definition we have

$$
\sum_{\delta \in \Delta_{\mathcal{R}_{P}, W_{P}}} \operatorname{dim}(\delta) d_{\mathcal{R}_{P}, \delta}=1
$$

Recall Conjecture 2.27 stating that $d_{\mathcal{R}_{P}, \delta} \in \mathbb{Q}_{+}$.

We define an Hermitian inner product on $\operatorname{Pol}\left(\operatorname{End}\left(\mathcal{V}_{(\mathcal{K} \backslash \Xi)}\right)\right)$ as follows:

$$
\left(f_{1}, f_{2}\right)=\sum_{\mathcal{O}}|W(\mathcal{O})|^{-1} \int_{\mathcal{O}} \operatorname{tr}\left(f_{1}(\omega)^{*} f_{2}(\omega)\right) d \mu_{P l}(\pi(\omega)),
$$

where the sum runs over a complete set of representatives for the association classes of orbits $\mathcal{O}$ (an association classes is an orbit under the action of $\mathcal{W} / \mathcal{K})$. Note that $f_{1}, f_{2}$ are in fact $K_{\delta}$-equivariant sections over the covering $\tilde{\mathcal{O}} \rightarrow \mathcal{O}$. The expression $\operatorname{tr}\left(f_{1}(\xi)^{*} f_{2}(\xi)\right)$ is independent of a choice of $\xi \in \tilde{\mathcal{O}}$ such that $K_{\delta} \xi=\omega$. The common value is denoted by $\operatorname{tr}\left(f_{1}(\omega)^{*} f_{2}(\omega)\right)$.

Theorem 4.43. (Main Theorem)

(i) Let $\mathcal{O}$ be an orbit (a connected component of $\mathcal{K} \backslash \Xi$ ). We put $\hat{\mathfrak{C}}_{\mathcal{O}}^{\text {gen }}=[\pi]\left(W(\mathcal{O}) \backslash \mathcal{O}^{\text {gen }}\right) \subset \hat{\mathfrak{C}}$, and we denote its closure by $\mathfrak{C}_{\mathcal{O}} \subset \hat{\mathfrak{C}}$. Then $\mathfrak{C}_{\mathcal{O}_{1}}^{\text {gen }} \cap \mathfrak{C}_{\mathcal{O}_{2}}^{\text {gen }}=\emptyset$ unless $\mathcal{O}_{1}$ and $\mathcal{O}_{2}$ are in the same $\mathcal{W} / \mathcal{K}$-orbit, and

$$
\hat{\mathfrak{C}}^{\text {gen }}:=\cup \hat{\mathfrak{C}}_{\mathcal{O}}^{\text {gen }}
$$

(union over a complete set of representatives for the association classes of orbits) is a dense set in $\mathfrak{C}$, whose complement has measure zero.

(ii) The Plancherel measure of $\mathfrak{C}$ (i.e. the measure on $\hat{\mathfrak{C}}$ determined by the tracial state $\tau$ of $\mathfrak{C})$ is given on $\hat{\mathfrak{C}}_{\mathcal{O}}$ by equation (4.96). The decomposition of $\tau$ in irreducible, mutually distinct characters of $\mathfrak{C}$ is given by

$$
\tau=\sum_{\mathcal{O}} \int_{\omega \in W(\mathcal{O}) \backslash \mathcal{O}} \chi_{\pi(\omega)} d \mu_{P l}(\pi(\omega))
$$

(sum over a complete set of representatives for the association classes of orbits).

(iii) Equivalently, the algebra homomorphism $\mathcal{F}_{\mathcal{H}}$ (see (4.92) and (4.94)) is an isometry with respect to the inner product (4.98), 
and extends uniquely to an isomorphism of $\mathfrak{C} \times \mathfrak{C}$ modules

$$
\mathcal{F}: \mathfrak{H} \stackrel{\sim}{\rightarrow} L^{2}\left(\operatorname{End}\left(\mathcal{V}_{\Xi}\right)\right)^{\mathcal{W}} \simeq \bigoplus_{\mathcal{O}} L^{2}\left(\operatorname{End}\left(\mathcal{V}_{\mathcal{O}}\right)\right)^{W(\mathcal{O})}
$$

(sum over a complete set of representatives for the association classes of orbits).

Proof. (i) See Theorem 4.39. The complement of $\hat{\mathfrak{C}}^{\text {gen }}$ has measure zero by the argument in the last part of the proof of that theorem. The density follows since $\hat{\mathfrak{C}}$ is the support of the Plancherel measure (cf. Theorem 2.25).

(ii) By formula of Proposition 3.15(v) and Corollary 4.37 we have

$$
\tau=\int_{\mathcal{W} \backslash \Lambda^{r e g}} \chi_{m(\lambda)} d \nu(m(\lambda))
$$

We decompose $\chi_{m(\lambda)}$ according to Theorem 4.23(ii) to obtain

$$
\tau=\int_{\lambda \in \mathcal{W} \backslash \Lambda^{r e g}}\left|W^{P(\lambda)}\right|^{-1} \sum_{\delta \in \Delta_{\gamma(\lambda)}} d_{\mathcal{R}(\lambda), \delta} \chi_{\pi(\lambda \times \delta)} d \nu(m(\lambda))
$$

By Corollary 4.18 and Theorem 4.39 we have $\{[\pi](\lambda \times \delta)\}_{\delta \in \Delta_{\gamma}}=$ $[\pi]\left(p_{\Sigma}^{-1}(\mathcal{W} \lambda)\right)$. Thus (by Theorem 4.39) we can rewrite the integral as integral over $\mathcal{W} \backslash \Xi^{\text {reg }}$. When we use parameters and notations as explained in equation (4.96), and we express $d \nu$ according to Proposition 3.27 , we obtain

$$
\tau=\int_{\xi \in \mathcal{W} \backslash \Xi^{r e g}}\left|W^{P(\lambda)}\right|^{-1}\left|W_{0}\left(r t^{P}\right)\right| d_{\mathcal{R}(\xi), \delta(\xi)} \chi_{\pi(\xi)} d \nu_{L}\left(r t^{P}\right)
$$

According to our definition of $\mu_{P l}$ this is equal to

$$
\tau=\sum_{\mathcal{O}} \int_{\omega \in W(\mathcal{O}) \backslash \mathcal{O}^{\text {reg }}} \chi_{\pi(\omega)} d \mu_{P l}(\pi(\omega))
$$

This is a decomposition of $\tau$ in characters of inequivalent irreducible representations of $\mathfrak{C}$ (see Theorem 4.23(iii)). Hence this uniquely determines the Plancherel measure (by [15], Théorème 8.8.6) on $\hat{\mathfrak{C}}$. We conclude that $\mu_{P l}$ is equal to the Plancherel measure of $\mathfrak{C}$.

(iii) The equivalence of (ii) and (iii) is well known, see the proof of Theorem 4.25. It is allowed to use the formulation with $W(\mathcal{O})$ equivariant sections because of the unitarity and the regularity of intertwining operators (Theorem 4.33) and by Proposition 4.42.

Remark 4.44. In [13] it is shown that the $\hat{\mathfrak{C}}_{\mathcal{O}}$ are the components of $\hat{\mathfrak{C}}$. Moreover, $\mathcal{F}(\mathfrak{S})$ (see 6.2.2 for the definition of $\mathfrak{S}$ ) and $\mathcal{F}(\mathfrak{C}$ ) are determined in [13]. 
ON THE SPECTRAL DECOMPOSITION OF AFFINE HECKE ALGEBRAS 93

Corollary 4.45. Let $\mathcal{J}: L^{2}\left(\operatorname{End}\left(\mathcal{V}_{(\mathcal{K} \backslash \Xi)}\right)\right) \rightarrow \mathfrak{H}$ denote the adjoint of $\mathcal{F}$. Then $\mathcal{J} \mathcal{F}=\operatorname{Id}$ and $\mathcal{F} \mathcal{J}(f)=\bar{f}$ (see subsection 4.5.4).

Proof. By the isometry property of $\mathcal{F},(\mathcal{J F}(x), y)=(x, y)$ for all $x, y \in$ $\mathfrak{H}$. Whence the first assertion. It is clear that $\mathcal{J}(f)=\mathcal{J}(\bar{f})$. If $g \in L^{2}\left(\operatorname{End}\left(\mathcal{V}_{\Xi}\right)\right)^{\mathcal{W}}$ then $g=\mathcal{F}(x)$ for some $x \in \mathfrak{H}$. Thus $\mathcal{F} \mathcal{J}(g)=$ $\mathcal{F} \mathcal{J} \mathcal{F}(x)=\mathcal{F}(x)=g$ for $\mathcal{W}$-equivariant $g$. Hence $\mathcal{F} \mathcal{J}(f)=\mathcal{F} \mathcal{J}(\bar{f})=$ $\bar{f}$.

\section{Base change invariance of the residual algebra}

Thus far we have found the spectral decomposition for $\mathcal{H}$ in terms of the "residual degrees" $d_{\mathcal{R}_{L}, \delta}$ of the residual algebras $\overline{\mathcal{H}_{L}^{r}}$. We prove in this section that the residual algebras are independent of $\mathbf{q}$ (using the Convention 2.1), up to isomorphism of Hilbert algebras.

\subsection{Scaling of the root labels}

Let $r=s c \in T$ be fixed, with $s \in T_{u}$ and $c=\exp (\gamma)$ with $\gamma \in \mathfrak{t}$. Assume that $B \subset \mathfrak{t}_{\mathbb{C}}$ is an open ball centered around the origin such that the conditions 4.9 (with respect to $r \in T$ ) are satisfied.

The second condition implies that each connected component of the union $U:=W_{0}(r \exp (B))$ contains a unique element of the orbit $W_{0} r$. Given $u \in U$ there is a unique $r^{\prime}=s^{\prime} c^{\prime} \in W_{0} r$ such that $u \in r^{\prime} \exp (B)$. By (i) there is a unique $b \in B$ such that $u=s^{\prime} c^{\prime} \exp (b)=s^{\prime} \exp \left(b+\gamma^{\prime}\right)$. Now let $\epsilon \in(0,1]$ be given. We define an analytic map $\sigma_{\epsilon}$ on $U$ by

$$
\sigma_{\epsilon}(u):=s^{\prime} \exp \left(\epsilon \log \left(\left(s^{\prime}\right)^{-1} u\right)\right)=s^{\prime} \exp \left(\epsilon\left(b+\gamma^{\prime}\right)\right) .
$$

Lemma 5.1. The map $\sigma_{\epsilon}$ is an analytic, $W_{0}$-equivariant diffeomorphism from $U$ onto $U_{\epsilon}:=W_{0}\left(s c^{\epsilon} \exp (\epsilon B)\right)$. The inverse of $\sigma_{\epsilon}$ will be denoted by $\sigma_{1 / \epsilon}$.

Proof. On the connected component $r^{\prime} \exp (B)$ the map $\sigma_{\epsilon}$ is equal to $\sigma_{\epsilon}=\mu_{s^{\prime}} \circ \exp \circ M_{\epsilon} \circ \log \circ \mu_{\left(s^{\prime}\right)^{-1}}$ where $\mu_{s^{\prime}}$ is the multiplication in $T$ by $s^{\prime}$, and $M_{\epsilon}$ is the multiplication in $\mathfrak{t}_{\mathbb{C}}$ by $\epsilon$. These are all analytic diffeomorphisms, because of condition (i). The $W_{0}$ equivariance follows from the fact that log is well defined (and thus equivariant, since exp is equivariant) from $W_{0} \exp (B+\gamma)$ to $W_{0}(B+\gamma)$, and that $M_{\epsilon}$ is $W_{0^{-}}$ equivariant. This implies that for $w \in W_{0}, w \exp \left(\epsilon \log \left(\left(s^{\prime}\right)^{-1} u\right)\right)=$ 
$\exp \left(\epsilon \log \left(\left(w s^{\prime}\right)^{-1} w u\right)\right)$. It follows that

$$
\begin{aligned}
\sigma_{\epsilon}(w u) & =w s^{\prime} \exp \left(\epsilon \log \left(\left(w s^{\prime}\right)^{-1} w u\right)\right) \\
& =w s^{\prime} w \exp \left(\epsilon \log \left(\left(s^{\prime}\right)^{-1} u\right)\right) \\
& =w\left(\sigma_{\epsilon}(u)\right) .
\end{aligned}
$$

Lemma 5.2. Denote by $q^{\epsilon}$ the label function $q^{\epsilon}(s)=q(s)^{\epsilon}=\mathbf{q}^{\epsilon f_{s}}$, and denote by $\mathcal{H}_{q^{\epsilon}}$ the affine Hecke algebra with root datum $\mathcal{R}$ (same as the root datum of the affine Hecke algebra $\mathcal{H}=\mathcal{H}_{q}$ ), but with the labels $q$ replaced by $q^{\epsilon}$. Let $c_{\alpha, \epsilon} \in{ }_{\mathcal{F}} \mathcal{A}_{q^{\epsilon}} \subset \mathcal{F}_{\mathcal{F}} \mathcal{H}_{q^{\epsilon}}$ be the corresponding Macdonald $c$-functions. For every root $\alpha \in R_{1}$ we have:

$$
U \ni u \rightarrow\left(c_{\alpha, \epsilon}\left(\sigma_{\epsilon}(u)\right) c_{\alpha}(u)^{-1}\right)^{ \pm 1} \in \mathcal{A}^{a n}(U) .
$$

Proof. For $u$ in the connected component $r^{\prime} \exp (B)$ of $U$ we write $u=$ $s^{\prime} v$ with $v \in c^{\prime} \exp (B)$. We have

$$
\begin{aligned}
c_{\alpha, \epsilon}\left(\sigma_{\epsilon}(u)\right) c_{\alpha}(u)^{-1} & =\frac{\left(1+q_{\alpha^{\vee}}^{-\epsilon / 2} \alpha(v)^{-\epsilon / 2} \alpha\left(s^{\prime}\right)^{-1 / 2}\right)}{\left(1+q_{\alpha \vee}^{-1 / 2} \alpha(v)^{-1 / 2} \alpha\left(s^{\prime}\right)^{-1 / 2}\right)} \\
\times & \frac{\left(1-q_{\alpha \vee}^{-\epsilon / 2} q_{2 \alpha^{\vee}}^{-\epsilon} \alpha(v)^{-\epsilon / 2} \alpha\left(s^{\prime}\right)^{-1 / 2}\right)\left(1-\alpha(v)^{-1} \alpha\left(s^{\prime}\right)^{-1}\right)}{\left(1-q_{\alpha^{\vee}}^{-1 / 2} q_{2 \alpha^{\vee}}^{-1} \alpha(v)^{-1 / 2} \alpha\left(s^{\prime}\right)^{-1 / 2}\right)\left(1-\alpha(v)^{-\epsilon} \alpha\left(s^{\prime}\right)^{-1}\right)}
\end{aligned}
$$

We remind the reader of the convention Remark 3.1; in particular, the expression $\alpha\left(s^{\prime}\right)^{1 / 2}$ occurs only if $\alpha / 2 \in R_{0}$, in which case this expression stands for $(\alpha / 2)\left(s^{\prime}\right)$. If $\alpha / 2 \notin R_{0}$, we should reduce formula (5.4) to

$$
c_{\alpha, \epsilon}\left(\sigma_{\epsilon}(u)\right) c_{\alpha}(u)^{-1}=\frac{\left(1-q_{\alpha^{\vee}}^{-\epsilon} \alpha(v)^{-\epsilon} \alpha\left(s^{\prime}\right)^{-1}\right)\left(1-\alpha(v)^{-1} \alpha\left(s^{\prime}\right)^{-1}\right)}{\left(1-q_{\alpha^{\vee}}^{-1} \alpha(v)^{-1} \alpha\left(s^{\prime}\right)^{-1}\right)\left(1-\alpha(v)^{-\epsilon} \alpha\left(s^{\prime}\right)^{-1}\right)}
$$

By conditions (i) and (iii) it is clear that poles and zeroes of these functions will only meet $U$ if $\alpha\left(s^{\prime}\right)=1$ when $\alpha \in R_{0} \cap R_{1}$ or $\alpha\left(s^{\prime}\right)= \pm 1$ if $\alpha \in 2 R_{0}$. In these cases the statement we want to prove reduces to the statement that the function

$$
f(x):=\frac{1-\exp (-\epsilon x)}{1-\exp (-x)}
$$

is holomorphic and invertible on the domain $x \in p+\alpha\left(\gamma^{\prime}+B\right)$, where $p$ is a real number and $\alpha \in R_{0}$. By condition (i) both the denominator and the numerator of $f$ have a zero in this domain only at $x=0$ (if this belong to the domain), and this zero is of order 1 both for the numerator and the denominator. The desired result follows. 
Recall Theorem 4.6. This result tells us that the structure of the algebra with coefficients in the locally defined meromorphic functions on $U$ is independent of the root labels. We will now show that the subalgebra with analytic coefficients (defined locally on $U$ ) is invariant for scaling transformations.

Theorem 5.3. The map

$$
\begin{aligned}
j_{\epsilon}: \mathcal{H}^{m e}(U) & \mapsto \mathcal{H}_{q^{\epsilon}}^{m e}\left(U_{\epsilon}\right) \\
\sum_{w \in W_{0}} f_{w} \iota_{w}^{0} & \mapsto \sum_{w \in W_{0}}\left(f_{w} \circ \sigma_{1 / \epsilon}\right) \iota_{w, \epsilon}^{0}
\end{aligned}
$$

defines an isomorphism of $\mathbb{C}$-algebras, with the property that $j_{\epsilon}\left(\mathcal{F}^{m e}(U)\right)$ $=\mathcal{F}_{q^{\epsilon}}^{m e}\left(U_{\epsilon}\right)$ and $j_{\epsilon}\left(\mathcal{A}^{m e}(U)\right)=\mathcal{A}_{q^{\epsilon}}^{m e}\left(U_{\epsilon}\right)$. Moreover (and most significantly), $j_{\epsilon}\left(\mathcal{H}^{a n}(U)\right)=\mathcal{H}_{q^{\epsilon}}^{a n}\left(U_{\epsilon}\right)$.

Proof. The map $j_{\epsilon}$ as defined above is clearly a $\mathbb{C}$-linear isomorphism by Theorem 4.6. It is an algebra homomorphism because we have

$$
\begin{aligned}
j_{\epsilon}\left(\sum_{u \in W_{0}} f_{u} \iota_{u}^{0} \sum_{v \in W_{0}} g_{v} \iota_{v}^{0}\right) & =j_{\epsilon}\left(\sum_{u, v \in W_{0}} f_{u} g_{v}^{u} \iota_{u v}^{0}\right) \\
& =\sum_{u, v \in W_{0}}\left(f_{u} \circ \sigma_{1 / \epsilon}\right)\left(g_{v}^{u} \circ \sigma_{1 / \epsilon}\right) \iota_{u v, \epsilon}^{0} \\
& =\sum_{u, v \in W_{0}}\left(f_{u} \circ \sigma_{1 / \epsilon}\right)\left(g_{v} \circ \sigma_{1 / \epsilon}\right)^{u} \iota_{u v, \epsilon}^{0} \\
& =\sum_{u, v \in W_{0}}\left(f_{u} \circ \sigma_{1 / \epsilon}\right) \iota_{u, \epsilon}^{0}\left(g_{v} \circ \sigma_{1 / \epsilon}\right) \iota_{v, \epsilon}^{0} \\
& =j_{\epsilon}\left(\sum_{u \in W_{0}} f_{u} \iota_{u}^{0}\right) j_{\epsilon}\left(\sum_{v \in W_{0}} g_{v} \iota_{v}^{0}\right)
\end{aligned}
$$

What remains is the proof that $j_{\epsilon}\left(\mathcal{H}^{a n}(U)\right)=\mathcal{H}_{q^{\epsilon}}^{a n}\left(U_{\epsilon}\right)$. Notice that $\mathcal{H}^{a n}(U)$ is the subalgebra generated by $\mathcal{A}^{a n}(U)$ and the elements $T_{s}$ where $s=s_{\alpha}$ with $\alpha \in R_{1}$. The $j_{\epsilon}$-image of $\mathcal{A}^{\text {an }}(U)$ equals $\mathcal{A}_{q^{\epsilon}}^{a n}\left(U_{\epsilon}\right)$ since $\sigma_{\epsilon}$ is an analytic diffeomorphism. To determine the image of $T_{s}$ we use formula Lemma 2.27(2) of [37], applied to the situation $W_{0}=\{e, s\}$. This tells us that

$$
\left(1+T_{s}\right)=q_{\alpha \vee} q_{2 \alpha} \vee c_{\alpha}\left(1+\iota_{s}^{0}\right) .
$$

Hence we see that

$$
\begin{aligned}
j_{\epsilon}\left(T_{s}\right) & =q_{\alpha^{\vee}} q_{2 \alpha^{\vee}}\left(c_{\alpha} \circ \sigma_{1 / \epsilon}\right)\left(1+\iota_{s, \epsilon}^{0}\right)-1 \\
& =q_{\alpha \vee}^{1-\epsilon} q_{2 \alpha \vee}^{1-\epsilon}\left(c_{\alpha} \circ \sigma_{1 / \epsilon}\right) c_{\alpha, \epsilon}^{-1}\left(1+T_{s, \epsilon}\right)-1 .
\end{aligned}
$$

By Lemma 5.2 it is clear that this is indeed in $\mathcal{H}_{q^{\epsilon}}^{a n}\left(U_{\epsilon}\right)$, and that these elements together with $\mathcal{A}_{q^{\epsilon}}^{a n}\left(m_{\epsilon}(U)\right)$ generate $\mathcal{H}_{q^{\epsilon}}^{a n}\left(U_{\epsilon}\right)$. 


\subsection{Application to the residual algebras}

In order to prove that the residual algebras $\overline{\mathcal{H}^{t}}$ are invariant for the scaling transformation $\mathbf{q} \rightarrow \mathbf{q}^{\epsilon}$ it suffices to consider the case $\overline{\mathcal{H}^{r}}$ for a residual point $r \in T$. This follows from Theorem 4.23, expressing $\chi_{t}$ in terms of characters induced from discrete series characters of proper parabolic subalgebras.

When $r=s c \in T$ is a residual point, the state $\chi_{r}$ has a natural extension to the localized algebras $\mathcal{H}^{a n}(U)$ where $U=W_{0} r \exp (B)$, with $B$ an open ball in $\mathfrak{t}_{\mathbb{C}}$ satisfying the conditions 4.9 with respect to the point $r \in T$. Because the radical $\operatorname{Rad}_{r}^{a n}(U)$ of the bitrace $(x, y)_{r}:=\chi_{r}\left(x^{*} y\right)$ on $\mathcal{H}^{a n}(U)$ is contained in the maximal ideal $\mathcal{I}_{r}^{a n}(U)$ of functions in the center $\mathcal{Z}^{a n}(U)$ which vanish in the orbit $W_{0} r$, we clearly have

$$
\overline{\mathcal{H}^{r}}=\mathcal{H}^{a n}(U) / \operatorname{Rad}_{r}^{a n}(U) .
$$

The structure of this algebra as a Hilbert algebra is given by the bitrace defined by $\chi_{r}$. Therefore, we need to prove independence of $\chi_{r}$ for the scaling transformation. We start with a simple lemma:

Lemma 5.4. Let $h \in \mathcal{H}^{a n}(U)$ We have

$$
\frac{E_{q^{\epsilon}, \sigma_{\epsilon}(t)}\left(j_{\epsilon}(h)\right)}{q^{\epsilon}\left(w_{0}\right) \Delta\left(\sigma_{\epsilon}(t)\right)}=\frac{E_{t}(h)}{q\left(w_{0}\right) \Delta(t)}
$$

Proof. For all $x \in X$ we have

$$
\begin{aligned}
E_{q^{\epsilon}, \sigma_{\epsilon}(t)}\left(j_{\epsilon}\left(\theta_{x} h\right)\right) & =E_{q^{\epsilon}, \sigma_{\epsilon}(t)}\left(j_{\epsilon}\left(\theta_{x}\right) j_{\epsilon}(h)\right) \\
& =\left(x \circ \sigma_{1 / \epsilon}\right)\left(\sigma_{\epsilon}(t)\right) E_{q^{\epsilon}, \sigma_{\epsilon}(t)}\left(j_{\epsilon}(h)\right) \\
& =x(t) E_{q^{\epsilon}, \sigma_{\epsilon}(t)}\left(j_{\epsilon}(h)\right),
\end{aligned}
$$

showing that the left hand side has the correct eigenvalue for multiplication of $h$ by $\theta_{x}$ on the left. For the multiplication of $h$ by $\theta_{x}$ on the right a similar computation holds. This shows, in view of Lemma 4.21 and [37], Proposition 2.23(3) that, for regular $t$ and outside the union of all residual cosets, the left and the right hand side are equal up to normalization. But both the left and the right hand side are equal to 1 if $h=T_{e}=1$. Hence generically in $t$, we have the desired equality. Since both expressions are holomorphic in $t$, the result extends to all $t \in T$.

Lemma 5.5. Let $\epsilon \in(0,1]$ be given. We have, for all $h \in \mathcal{H}^{a n}(U)$,

$$
\chi_{q^{\epsilon}, \sigma_{\epsilon}(r)}\left(j_{\epsilon}(h)\right)=\chi_{r}(h) .
$$

Proof. Take a neighborhood $U=W_{0} r \exp (B)$ with $B$ satisfying conditions 4.9 relative to $r$. Let $\cup \xi \in \mathcal{H}_{n}(U)$ denote the $n$-cycle defined 
ON THE SPECTRAL DECOMPOSITION OF AFFINE HECKE ALGEBRAS 97

by $\cup \xi=\cup_{r^{\prime} \in W_{0} r} \xi_{r^{\prime}}$. In view of Proposition 3.7, Definition 3.16 and Definition 3.22 we see that, for all $h \in \mathcal{H}$,

$$
\nu\left(\left\{W_{0} r\right\}\right) \chi_{r}(h)=\int_{\cup \xi}\left(\frac{E_{t}(h)}{q\left(w_{0}\right) \Delta(t)}\right) \frac{d t}{q\left(w_{0}\right) c(t) c\left(t^{-1}\right)}
$$

Let $r^{\prime} \in W_{0} r$. The scaling operation sends the root labels $q$ to $q^{\epsilon}$, and follows the corresponding path of $\epsilon \rightarrow \sigma_{\epsilon}\left(r^{\prime}\right)$ of the residual point $r^{\prime}$. Obviously the position of $t_{0}$ (in equation (3.1)) relative to $\mathcal{L}^{\left\{\sigma_{\epsilon}\left(r^{\prime}\right)\right\}}$ is independent of $\epsilon$. And also, the position of $e$ relative to the facets of the dual configuration $\mathcal{L}_{\left\{\sigma_{\epsilon}\left(r^{\prime}\right)\right\}}$ is independent of $\epsilon$, since the effect of the scaling operation on $\mathcal{L}_{\left\{r^{\prime}\right\}} \subset T_{r s}$ simply amounts to the application of the map $c \rightarrow c^{\epsilon}$. In view of Proposition 3.10 and Proposition 3.13, we can take the cycle $\sigma_{\epsilon}(\cup \xi) \in H_{n}\left(\sigma_{\epsilon}(U)\right)$ in order to define the state $\chi_{\sigma_{\epsilon}(r)}$ of $\mathcal{H}_{q^{\epsilon}}^{a n}\left(\sigma_{\epsilon}(U)\right)$. In other words, we have, for $h \in \mathcal{H}^{a n}(U)$,

$$
\begin{aligned}
\nu_{q^{\epsilon}}\left(\left\{W_{0} \sigma_{\epsilon}(r)\right\}\right) \chi_{q^{\epsilon}, \sigma_{\epsilon}(r)}\left(j_{\epsilon}(h)\right) \\
=\int_{\sigma_{\epsilon}(\cup \xi)}\left(\frac{E_{t}\left(j_{\epsilon}(h)\right)}{q^{\epsilon}\left(w_{0}\right) \Delta(t)}\right) \frac{d t}{q^{\epsilon}\left(w_{0}\right) c_{\epsilon}(t) c_{\epsilon}\left(t^{-1}\right)} \\
=\int_{\cup \xi}\left(\frac{E_{\sigma_{\epsilon}(t)}\left(j_{\epsilon}(h)\right)}{q^{\epsilon}\left(w_{0}\right) \Delta\left(\sigma_{\epsilon}(t)\right)}\right) \frac{d\left(\sigma_{\epsilon}(t)\right)}{q^{\epsilon}\left(w_{0}\right) c_{\epsilon}\left(\sigma_{\epsilon}(t)\right) c_{\epsilon}\left(\sigma_{\epsilon}\left(t^{-1}\right)\right)} \\
=\int_{\cup \xi}\left(\frac{E_{t}(h)}{q\left(w_{0}\right) \Delta(t)}\right) \phi_{\epsilon}(t) \frac{d t}{q\left(w_{0}\right) c(t) c\left(t^{-1}\right)},
\end{aligned}
$$

where

$$
\phi_{\epsilon}(t):=\frac{\epsilon^{n} q\left(w_{0}\right) c(t) c\left(t^{-1}\right)}{q^{\epsilon}\left(w_{0}\right) c_{\epsilon}\left(\sigma_{\epsilon}(t)\right) c_{\epsilon}\left(\sigma_{\epsilon}\left(t^{-1}\right)\right)} .
$$

By Lemma 5.2, the function $t \rightarrow \phi_{\epsilon}$ extends, for all $\epsilon \in(0,1]$, to a regular holomorphic function on $U$. Clearly, $\phi_{\epsilon}$ is $W_{0}$-invariant. In other words, $\phi_{\epsilon}$ is an element of $\mathcal{Z}^{a n}(U)$. Its value in $W_{0} r$ can be computed easily, if we keep in mind that the index $i_{\left\{r^{\prime}\right\}}=n$ (by Theorem 7.10 applied to the residual coset $\left.r^{\prime}\right)$. We obtain, by a straightforward computation:

$$
\phi_{\epsilon}\left(W_{0} r\right)=\frac{m_{q^{\epsilon},\left\{\sigma_{\epsilon}(r)\right\}}(r)}{m_{r}(r)}=\frac{\nu_{q^{\epsilon}}\left(\left\{W_{0} \sigma_{\epsilon}(r)\right\}\right)}{\nu\left(\left\{W_{0} r\right\}\right)} .
$$

We now continue the computation which we began in equation (5.16), using the fact that $\phi_{\epsilon} \in \mathcal{Z}^{a n}(U)$ and the fact that $\chi_{r}$ extends uniquely to $\mathcal{H}^{a n}(U)$ in such a way that for all $\phi \in \mathcal{Z}^{a n}(U)$ and $h \in \mathcal{H}^{a n}(U)$, 
$\chi_{r}(\phi h)=\phi(r) \chi(h)$. We get

$$
\begin{aligned}
\nu_{q^{\epsilon}}\left(\left\{W_{0} \sigma_{\epsilon}(r)\right\}\right) \chi_{q^{\epsilon}, \sigma_{\epsilon}(r)}\left(j_{\epsilon}(h)\right) & =\nu\left(\left\{W_{0} r\right\}\right) \chi_{r}\left(\phi_{\epsilon} h\right) \\
& =\nu_{q^{\epsilon}}\left(\left\{W_{0} \sigma_{\epsilon}(r)\right\}\right) \chi_{r}(h) .
\end{aligned}
$$

This gives the desired result.

Theorem 5.6. The "base change" isomorphism $j_{\epsilon}$ induces an isomorphism

$$
\bar{j}_{\epsilon}: \overline{\mathcal{H}^{r}} \stackrel{\sim}{\longrightarrow} \overline{\mathcal{H}_{q^{\epsilon}}^{\sigma_{\epsilon}(r)}}
$$

of Hilbert algebras. In particular, the positive constants $d_{\mathcal{R}_{P}, \delta}$ (in the notation of Theorem 4.23, see also equation (4.96)) (in Corollary 3.32 these constant were denoted by $\left.d_{r, i}\right)$ are independent of $\mathbf{q}$.

Proof. This is an immediate consequence of the previous lemma.

\section{Applications and closing remarks}

\subsection{Formation of L-packets of unipotent representations}

Let $F$ be a nonarchimedean local field, and let $G$ be a split simple algebraic group of adjoint type defined over $F$. We denote by $\mathcal{G}$ the group of $F$-rational points in $G$. The finite set of irreducible unipotent discrete series representations of $\mathcal{G}$ is by definition the disjoint union of the discrete series constituents of induced representations of the form $\sigma_{\mathcal{P}}^{\mathcal{G}}$, where $\mathcal{P}$ is a parahoric subgroup of $\mathcal{G}, \sigma$ is a cuspidal unipotent representation of the Levi quotient $L:=\mathcal{P} / \mathcal{U}_{\mathcal{P}}$ of $\mathcal{P}$, and the union is taken over a complete set of representatives of conjugacy classes of pairs $(\mathcal{P}, \sigma)$.

The formal dimension is an effective tool to partition these unipotent discrete series representations into L-packets. This observation is due to Reeder [39]. He conjectured that the formal dimensions of the unipotent discrete series representations of $\mathcal{G}$ within one unipotent discrete series L-packet are proportional, with a rational ratio of proportionality independent of $F$, and used this in [39] to form the unipotent L-packets for groups of small rank.

It is known [36] that the endomorphism algebra $\mathcal{H}(\mathcal{G}, \mathcal{P}, \sigma)$ of $\sigma_{\mathcal{P}}^{\mathcal{G}}$ has the structure of an affine Hecke algebra, whose root datum and root labels depend only on $\mathcal{P}$. The root labels are integral powers $\mathbf{q}^{n_{a}}$ of the cardinality $\mathbf{q}$ of the residue field of $F$ (cf. [28]), and are explicitly known. Moreover, if we define a trace functional $\operatorname{Tr}$ on $H(\mathcal{G}, \mathcal{P}, \sigma)$ by 
$\operatorname{Tr}(f):=\operatorname{Tr}\left(f(e), V_{\sigma}\right)$, this corresponds to the trace $\tau$ studied in this paper by the formula

$$
\operatorname{Tr}=\operatorname{Vol}(\mathcal{P})^{-1} \operatorname{dim}\left(V_{\sigma}\right) \tau .
$$

Thus there is a bijection between the set of discrete series representations of $H(\mathcal{G}, \mathcal{P}, \sigma)$ (in the sense of this paper) and the unipotent discrete series representations arising from the pair $(\mathcal{P}, \sigma)$. The formal dimension of such a discrete series representation of $\mathcal{G}$ is then equal to the formal dimension of the corresponding discrete series representation of the affine Hecke algebra $H(\mathcal{G}, \mathcal{P}, \sigma)$, but with its trace Tr normalized by equation (6.1).

In [19] we computed the formal dimension of the discrete series representations of the "anti-spherical" subalgebra of the affine Hecke algebra, i.e. the commutative subalgebra $e_{-} \mathcal{H} e_{-}=e_{-} \mathcal{Z}$, where $e_{-}$denotes the idempotent of $\mathcal{H}_{0}$ corresponding to the sign representation (see Subsection 6.3). The formula we obtained was expressed entirely in terms of the central character of the representation, the root datum and the root labels. We conjectured in [19] that our formula would also hold for the full affine Hecke algebra. For the group of type $E_{8}$, we showed that this, in combination with Reeder's conjecture, leads to a partitioning of the unipotent discrete series L-packets which is in agreement with Lusztig's conjecture [25] for the Langlands parameters of the members of these packets. In other words, the formal dimension seems to be a sufficient criterion to separate the L-packets of unipotent representations in the case $E_{8}$. Theorem 4.43 of this paper proves the conjecture in [19] mentioned above.

Reeder [40] proves an exact formula for the formal dimension of the unipotent discrete series representation of all split exceptional groups, based on a result of Schneider and Stuhler [42]. In this approach one first represents the formal dimension by an alternating sum of rational functions (depending on the $K$-types (cf. Subsection 6.6)), rather than the product formula which we have obtained. On the other hand, there are no intractable constants such as the constant $d_{\delta}$ in our formula. Using his previous work on non-standard intertwining operators for affine Hecke algebra modules and Theorem 3.25 of the present paper, Reeder gave the precise partitioning of the unipotent discrete series for exceptional groups into L-packets, in complete agreement with Lusztig's conjecture mentioned above.

Recently, Lusztig [29] established the partitioning of unipotent discrete series representations into L-packets if $G$ is split over an unramified extension of $F$. This is based on a different approach. It is worth mentioning that this classification includes a geometric parametrization 
of the set $\Delta_{W_{0} r}$ of discrete series representations with central character $W_{0} r$ if the affine Hecke algebra arises as an endomorphism algebra of an induced representation of the form $\sigma_{\mathcal{P}}^{\mathcal{G}}$.

\subsection{Operator norm estimate and the Schwartz completion}

6.2.1. Uniform norm estimate. We know that the generators $N_{i}:=$ $q\left(s_{i}\right)^{-1 / 2} T_{i}$ satisfy

$$
\left\|N_{i}\right\|_{o}=\max \left\{q\left(s_{i}\right)^{ \pm 1 / 2}\right\}
$$

Therefore we have the trivial estimate $\left\|N_{w}\right\|_{o} \leq \max \left\{q(w)^{ \pm 1 / 2}\right\}$. By the spectral decomposition it is easy to see that the operator norm $\left\|N_{w}\right\|_{o}$ is actually bounded by a polynomial in $\mathcal{N}(w)$ :

Theorem 6.1. Let $\mathbf{q}>1$ be fixed.

(i) There exist constants $C \in \mathbb{R}_{+}$and $d \in \mathbb{N}$ such that for all $w \in W$

$$
\left\|N_{w}\right\|_{o} \leq C(1+\mathcal{N}(w))^{d}
$$

(ii) For a residual point $r \in T$, let $\Delta_{W_{0} r}:=\Delta_{\mathcal{R}, W_{0} r}$ denote the collection of all discrete series representations with central character $W_{0} r$. Denote by $\|x\|_{d s}$ the operator norm of the left multiplication by $x \in \mathcal{H}$, restricted to the finite dimensional subspace

$$
\mathfrak{H}_{d s}:=\oplus_{W_{0} r} \oplus_{\pi \in \Delta_{W_{0}}} \operatorname{End}(\pi) \subset \mathfrak{H} .
$$

Then there exist constants $C, \epsilon>0$ such that

$$
\left\|N_{w}\right\|_{d s} \leq C \mathbf{q}^{-\epsilon l(w)} .
$$

Proof. (i) As was explained in the proof of Lemma 2.20, it is sufficient to prove this statement for $w=x \in\left(Z_{X}+Q\right) \cap X^{+}$. Recall that in this case $N_{x}=\theta_{x}$. We have

$$
\left\|\theta_{x}\right\|_{o}^{2}=\left\|\theta_{x}^{*} \theta_{x}\right\|_{o}=\sup \left\{\sigma\left(\pi\left(T_{w_{0}} \theta_{-w_{0} x} T_{w_{0}}^{-1} \theta_{x}\right)\right) \mid \pi \in \hat{\mathfrak{C}}\right\},
$$

where $\sigma(A)$ denotes the spectral radius of $A$. According to Theorem 4.43 , the spectrum $\hat{\mathfrak{C}}$ equals the union of the compact sets $\hat{\mathfrak{C}}_{\mathcal{R}_{P}, W_{P} r, \delta}$.

This set is by definition the closure in $\hat{\mathfrak{C}}$ of the set $\pi\left(\Gamma_{\mathcal{R}_{P}, W_{P} r, \delta}^{g e n}\right)$. It is well known that for any $h \in \mathcal{H}$, the map $\pi \rightarrow\|\pi(h)\|_{o}$ is lower semicontinuous as a function of $\pi \in \hat{\mathfrak{C}}$ (cf. [16], VII, Proposition 1.14). Since there are only finitely many triples $\left(R_{P}, W_{P} r, \delta\right)$, it is sufficient to show that there exist constants $C, d$ such that the spectral radius of

$$
\pi\left(\mathcal{R}_{P}, W_{P} r, \delta, t^{P}\right)\left(T_{w_{0}} \theta_{-w_{0} x} T_{w_{0}}^{-1} \theta_{x}\right)
$$


ON THE SPECTRAL DECOMPOSITION OF AFFINE HECKE ALGEBRAS 101

is bounded by $C(1+\mathcal{N}(x))^{d}$, uniformly in $t^{P}$.

The roots of a monic polynomial are bounded by the sum of the absolute values of the coefficients of the equation (including the top coefficient 1). Hence the spectral radius of an $m \times m$ matrix $A$ is bounded by a polynomial of degree $m$ in $\max (A):=\max \left\{\left|a_{i, j}\right| \mid 1 \leq\right.$ $i, j \leq m\}$. Since $m \leq\left|W_{0}\right|$ it is sufficient to show that there exists a suitable basis for the parameter family $\pi\left(\mathcal{R}_{P}, W_{P} r, \delta, t^{P}\right)$ (with $t^{P} \in$ $T_{u}^{P}$ ) of representations, in which the matrix coefficients of the $\theta_{x}$ (with $\left.x \in\left(Z_{X}+Q\right) \cap X^{+}\right)$are uniformly bounded by $C(1+\mathcal{N}(x))^{d}$ for suitable constants $C$ and $d$.

As in the proof of Proposition 4.20, there exists a basis $T_{w_{i}} \otimes\left(v_{j}\right)$ of $V_{\pi}=\mathcal{H}\left(W^{P}\right) \otimes V$, the representation space of $\pi\left(\mathcal{R}_{P}, W_{P} r, \delta, t^{P}\right)$, such that the $\theta_{x}(x \in X)$ simultaneously act by means of upper triangular matrices in this basis. Moreover, by Proposition 4.20 it is clear that the diagonal elements are bounded in norm by 1 when $x \in X^{+}$. By the compactness of $T_{u}^{P}$ we conclude that there exists, for each $x \in X^{+}$, an unipotent upper triangular matrix $U_{x}$ with positive coefficients such that every matrix coefficient of $M_{x}\left(t^{P}\right):=\pi\left(\mathcal{R}_{P}, W_{P} r, \delta, t^{P}\right)\left(\theta_{x}\right)$ in the above basis is uniformly (in $t^{P}$ ) bounded by the corresponding matrix coefficient of $U_{x}$.

Let us denote by $P$ the set of $n \times n$ matrices with non-negative entries, and introduce the notation $|A|=\left(\left|A_{i, j}\right|\right)_{i, j}$ for complex matrices $A$. Introduce a partial ordering in $P$ by defining $A \leq B$ if and only if $B-A \in P$. Since $P$ is a semigroup for matrix multiplication, it is clear that if $A, B$ and $C$ are in $P$ and $A \leq B$, then $A C \leq B C$. In addition we have the rule $|A B| \leq|A||B|$ for arbitrary complex matrices $A$ and $B$.

Let $x_{1}, \ldots, x_{m}$ denote a set of $\mathbb{Z}_{+}$-generators for the cone $Q^{+}$, and let moreover $x_{m+1}, \ldots, x_{N}$ be a basis of $Z_{X}$. Put $M_{i, \epsilon}\left(t^{P}\right):=M_{\epsilon x_{i}}\left(t^{P}\right)$ for $1 \leq i \leq N, \epsilon= \pm 1$ with $\epsilon=1$ if $i \leq m$. We can thus find an upper triangular unipotent matrix $U \in \bar{P}$ such that for all $i, \epsilon, t^{P}$ : $\left|M_{i, \epsilon}\left(t^{P}\right)\right| \leq U$. If we write $x=\sum_{i} l_{i} x_{i}$ with $l_{i} \geq 0$ if $i \leq m$, then

$$
\begin{aligned}
\mathcal{N}(x) & =x\left(2 \rho^{\vee}\right)+\left\|\sum_{i>m} l_{i} x_{i}\right\| \\
& =\sum_{i \leq m} l_{i} x_{i}\left(2 \rho^{\vee}\right)+\left\|\sum_{i>m} l_{i} x_{i}\right\|
\end{aligned}
$$

with $x_{i}\left(2 \rho^{\vee}\right) \geq 1$ if $i \leq m$. From this we see that there exists a constant $K$ independent of $x$ such that $\alpha:=\sum\left|l_{i}\right| \leq K \mathcal{N}(x)$. Thus, with $\log (U)$ 
the nilpotent logarithm of $U$, and $l_{i}=\epsilon(i)\left|l_{i}\right|$ :

$$
\begin{aligned}
\max \left(M_{x}\left(t^{P}\right)\right) & =\max \left(\left|M_{1}^{l_{1}}\left(t^{P}\right) \ldots M_{N}^{l_{N}}\left(t^{P}\right)\right|\right) \\
& \leq \max \left(\left|M_{1, \epsilon(1)}^{\left|l_{1}\right|}\left(t^{P}\right)\right| \ldots\left|M_{N, \epsilon(N)}^{\left|l_{N}\right|}\left(t^{P}\right)\right|\right) \\
& \leq \max \left(U^{\alpha}\right) \\
& \leq \max (\exp (\alpha \log (U))) \\
& \leq \sum_{i=0}^{d} \max \left(\log (U)^{i}\right) \alpha^{i} / i ! \\
& \leq c_{U}(1+\mathcal{N}(x))^{d}
\end{aligned}
$$

where $c_{U}$ is a constant depending on $U$ only, and $d$ is the degree of the polynomial function $\alpha \rightarrow \exp (\alpha \log (U))$. This finishes the proof.

(ii) As in the proof of (i), but we restrict ourselves to the (finitely many) discrete series representations. This implies that we can find $U \in P$ unipotent and $\epsilon>0$ such that for all $i,\left|M_{i}\right| \leq \mathbf{q}^{-2 \epsilon} U$. Inserting this in the inequalities (6.8) we find that the matrix entries of $M_{x}$ are bounded by $C \mathbf{q}^{-\epsilon l(x)}$, with $C$ independent of $x \in X^{+}$. Hence the spectral radius of $\mathbf{q}^{2 \epsilon l(x)} M_{x}^{*} M_{x}$ is uniformly bounded for $x \in X^{+}$, proving the desired estimate.

Corollary 6.2. $\hat{\mathfrak{C}}$ consists only of tempered representations.

Proof. The character $\chi_{\pi}$ of $\pi \in \hat{\mathfrak{C}}$ is a positive trace, and thus satisfies the inequality $\left|\chi_{\pi}(x)\right| \leq \chi_{\pi}(1)\|x\|_{o}$ by Corollary 2.17. By Casselman's criterion Lemma 2.20 and Theorem 6.1 this implies that $\pi$ is tempered.

Proposition 6.3. The trivial representation $\pi_{\text {triv }}\left(T_{w}\right)=q(w)$ is tempered if and only if the point $r_{\text {triv }} \in T_{\text {rs }}$ defined by $\forall \alpha \in F_{0}: \alpha\left(r_{\text {triv }}\right)=$ $q_{\alpha \vee / 2}^{1 / 2} q_{\alpha \vee}$ satisfies $r_{\text {triv }} \in \overline{T_{r s}^{-}}$. It is discrete series if and only if $r_{\text {triv }} \in$ $T_{r s}^{-}$. The Steinberg representation $\pi_{S t}\left(T_{w}\right)=(-1)^{l(w)}$ is tempered if and only if $r_{\text {triv }}^{-1}:=r_{S t} \in \overline{T_{r s}^{-}}$, and discrete series if and only if $r_{S t} \in T_{r s}^{-}$.

Proof. This is well known, and follows easily by the remark that the restriction to $\left\{\theta_{x} \mid x \in X\right\}$ of the trivial representation is equal the square root of the Haar modulus $\delta$ (see e.g. [37], Corollary 1.5): $\pi_{\text {triv }}\left(\theta_{x}\right)=q(x)^{1 / 2}=\delta^{1 / 2}(x):=x\left(r_{\text {triv }}\right)$. Now apply the Casselman criteria Lemma 2.20. Similar remarks apply to the case of the Steinberg representation.

Corollary 6.4. If the trivial representation extends to $\mathfrak{C}$ then $r_{\text {triv }} \in$ $\overline{T_{r s}^{-}}$. If the Steinberg representation extends to $\mathfrak{C}$ then $r_{\text {triv }} \in \overline{T_{r s}^{+}}$. 
ON THE SPECTRAL DECOMPOSITION OF AFFINE HECKE ALGEBRAS 103

Proof. Use Corollary 6.2 and Proposition 6.3.

6.2.2. The Schwartz completion of $\mathcal{H}$. Using Theorem 6.1 we now define a Fréchet completion of $\mathcal{H}$. For all $n \in \mathbb{N}$ we define a norm $p_{n}$ on $\mathcal{H}$ by

$$
p_{n}(h)=\max _{w \in W}\left|\left(N_{w}, h\right)\right|(1+\mathcal{N}(w))^{n}
$$

Here $\mathcal{N}$ denotes the norm function on $W$ which was defined by equation $(2.5)$.

Theorem 6.5. The functions $\tau$, * and the multiplication $\cdot$ of $\mathcal{H}$ are continuous with respect to the family of norms $p_{n}$.

Proof. The continuity of $\tau$ and $*$ is immediate from the definitions. So let us look at the multiplication. Let us write

$$
N_{u} N_{v}=\sum_{w} c_{u, v}^{w} N_{w}
$$

It is easy to see that $w(0)^{0}=u(0)^{0}+v(0)^{0}$ and that $l(w) \leq l(u)+l(v)$ if $c_{u, v}^{w} \neq 0$. Therefore

$$
c_{u, v}^{w} \neq 0 \Rightarrow \mathcal{N}(w) \leq \mathcal{N}(u)+\mathcal{N}(v),
$$

and by Theorem 6.1 , there exist constants $C, d$ such that for all $u, v$ and $w$ :

$$
\left|c_{u, v}^{w}\right| \leq C \min \left\{(1+\mathcal{N}(u))^{d},(1+\mathcal{N}(v))^{d}\right\}
$$

We put $D_{w}=\left\{(u, v) \in W \times W \mid c_{u, v}^{w} \neq 0\right\}$. It is easy to see that there exists a $b \in \mathbb{N}$ such that

$$
\sum_{u \in W} \frac{1}{(1+\mathcal{N}(u))^{b}}=\mu<\infty
$$

converges. By (6.11) we have that

$$
(1+\mathcal{N}(u))(1+\mathcal{N}(v)) \geq(1+\mathcal{N}(w))
$$

for all $(u, v) \in D_{w}$. Given $n \in \mathbb{N}$, let $k=\max \{b+d, n\}$. Using these remarks we see that for all $0 \neq x=\sum x_{u} N_{u}$ and $0 \neq y=\sum y_{v} N_{v}$ in 
$\mathcal{H}$ the following holds:

$$
\begin{aligned}
\frac{p_{n}(x y)}{p_{2 k}(x) p_{2 k}(y)} & =\frac{1}{p_{2 k}(x) p_{2 k}(y)} \max _{w}\left|\left(x y, N_{w}\right)\right|(1+\mathcal{N}(w))^{n} \\
& \leq \max _{w} \sum_{u, v \in D_{w}} \frac{\left|x_{u}\right|\left|y_{v}\right|}{p_{2 k}(x) p_{2 k}(y)}\left|c_{u, v}^{w}\right|(1+\mathcal{N}(w))^{n} \\
& \leq C \sup _{w} \sum_{u, v \in D_{w}} \frac{\min \left\{(1+\mathcal{N}(u))^{d},(1+\mathcal{N}(v))^{d}\right\}(1+\mathcal{N}(w))^{n}}{(1+\mathcal{N}(u))^{2 k}(1+\mathcal{N}(v))^{2 k}} \\
& \leq C \sup _{w} \sum_{u, v \in D_{w}} \frac{(1+\mathcal{N}(u))^{d}(1+\mathcal{N}(v))^{d}(1+\mathcal{N}(w))^{n-k}}{(1+\mathcal{N}(u))^{k}(1+\mathcal{N}(v))^{k}} \\
& \leq C \sum_{u, v}(1+\mathcal{N}(u))^{d-k}(1+\mathcal{N}(v))^{d-k} \\
& \leq \mu^{2} C
\end{aligned}
$$

This finishes the proof.

Notice that, by Theorem $6.1,\|x\|_{o} \leq C p_{d}(x)$ for all $x \in \mathcal{H}$. Therefore the completion of $\mathcal{H}$ with respect to the family of norms $p_{n}$ will be a subspace of $\mathfrak{C}$.

Definition 6.6. We define the Schwartz completion $\mathfrak{S}$ of $\mathcal{H}$ by

$$
\mathfrak{S}:=\left\{x=\sum_{w} x_{w} N_{w} \in \mathcal{H}^{*} \mid p_{n}(x)<\infty \forall n \in \mathbb{N}\right\} .
$$

We have $\mathcal{H} \subset \mathfrak{S} \subset \mathfrak{C}$, and $\mathfrak{S}$ is a *-subalgebra of $\mathfrak{C}$. $\mathfrak{S}$ is a nuclear Fréchet algebra with respect to the topology defined by the family of norms $p_{n}$. It comes equipped with continuous trace $\tau$ and antiinvolution *.

Corollary 6.7. (of definition) The topological dual $\mathfrak{S}^{\prime}$ is the space of tempered linear functionals on $\mathcal{H}$.

\subsection{A Hilbert algebra isomorphism; abelian subalgebras}

There exists a trace preserving $*$-algebra isomorphism

$$
\begin{aligned}
i: \mathcal{H}(\mathcal{R}, q) & \rightarrow \mathcal{H}\left(\mathcal{R}, q^{-1}\right) \\
N_{w} & \rightarrow(-1)^{l(w)} N_{w} .
\end{aligned}
$$

(see [19]). Clearly this induces a $*$-algebra isomorphism, and $\tau$ is respected. Thus $i$ induces an isomorphism of $C^{*}$-algebras $i: \mathfrak{C}(\mathcal{R}, q) \rightarrow$ 
ON THE SPECTRAL DECOMPOSITION OF AFFINE HECKE ALGEBRAS 105

$\mathfrak{C}\left(\mathcal{R}, q^{-1}\right)$, also respecting the traces. The corresponding homeomorphism $\hat{i}: \hat{\mathfrak{C}}\left(\mathcal{R}, q^{-1}\right) \rightarrow \hat{\mathfrak{C}}(\mathcal{R}, q)$ is therefore Plancherel measure preserving. Note that $i$ restricts to a (Plancherel measure preserving) *-isomorphism from the subalgebra $e_{+} \mathcal{H}(\mathcal{R}, q) e_{+}$(the spherical subalgebra) to the subalgebra $e_{-} \mathcal{H}\left(\mathcal{R}, q^{-1}\right) e_{-}$(the anti-spherical subalgebra) (see [19]).

6.3.1. The Plancherel measure of the center. The commutative subalgebras $e_{ \pm} \mathcal{H}(\mathcal{R}, q) e_{ \pm}$are both isomorphic as algebras to the center $\mathcal{Z}$ via the Satake isomorphism $\mathcal{Z} \ni z \rightarrow e_{ \pm} z \in e_{ \pm} \mathcal{H}(\mathcal{R}, q) e_{ \pm}$. These subalgebras are commutative Hilbert subalgebras of $\mathcal{H}(\mathcal{R}, q)$. They are in general not isomorphic as Hilbert algebras. The Hilbert algebra isomorphism $i$ restricts to an isomorphism $\mathcal{Z}(\mathcal{R}, q) \rightarrow \mathcal{Z}\left(\mathcal{R}, q^{-1}\right)$. Recall the $\nu$ is the Plancherel measure of $\overline{\mathcal{Z}} \subset \mathfrak{C}$. The above is reflected by the symmetry

Corollary 6.8. $\nu(t, q)=\nu\left(t, q^{-1}\right)$,

which can be verified directly (see Theorem 3.25 and Proposition 3.27).

The spherical algebra $e_{+} \mathcal{H}(\mathcal{R}, q) e_{+}$with $X=P$ (weight lattice) and $q(s)>1$ has a very important basis, uniquely defined by orthogonality and by a triangularity requirement with respect to the standard monomial basis $e_{+} m_{\lambda}$ (with $\lambda \in P^{+}$and $m_{\lambda}=\sum_{\mu \in W_{0} \lambda} t^{\mu} \in \mathcal{Z}$ ). In type $A$ these are the Hall-Littlewood polynomials. It would be interesting to study such orthogonal, triangular bases for the center $\mathcal{Z}$ as well.

\subsection{Central idempotents of $\mathfrak{C}$ and $\mathfrak{S}$}

Recall that $\hat{\mathfrak{C}}^{\mathfrak{}}=\cup \hat{\mathfrak{C}}_{\mathcal{O}}$ (union over a complete set of representatives of the association classes orbits). In Theorem 4.43 we have shown that two distinct closed subsets in $\hat{\mathfrak{C}}$ of the form $\hat{\mathfrak{C}}_{\mathcal{O}_{i}}(i=1,2)$ intersect in a subset of measure 0 . In fact more is true: according to [13], these closed subsets are the components of $\hat{\mathfrak{C}}$.

There is a bijection $I \rightarrow \hat{I}$ between the closed two-sided ideals of $\mathfrak{C}$ and the open subsets of $\hat{\mathfrak{C}}$. Hence the decomposition of $\hat{\mathfrak{C}}^{2}$ into components $\hat{\mathfrak{C}}_{\mathcal{O}}$ corresponds to the decomposition of $1 \in \mathfrak{C}$ as a sum of minimal central orthogonal idempotents $e_{\mathcal{O}}$ of $\mathfrak{C}$.

If $\mathcal{O}=\mathcal{K}_{\delta} \backslash\left(\Lambda_{\gamma} \times \delta\right), e_{\mathcal{O}} \in \mathfrak{C}$ is determined by

$$
\mathcal{F}\left(e_{\mathcal{O}}\right)(\pi)=\left\{\begin{array}{l}
\operatorname{Id}_{\mathrm{i}\left(\mathrm{V}_{\delta}\right)} \text { if }[\pi] \in \hat{\mathfrak{C}}_{\mathcal{O}} \\
0 \text { else },
\end{array}\right.
$$

where $\mathcal{F}$ is the isomorphism of Theorem 4.43 . 
In fact, the results of [13] on smooth wave packets even imply that $e_{\mathcal{O}} \in \mathfrak{S}$. We thus have the following decomposition of the unit element in central, Hermitian, mutually orthogonal, minimal idempotents of $\mathfrak{S}$ :

$$
1=\sum_{\mathcal{O}} e_{\mathcal{O}}
$$

Theorem 6.9. $\quad$ (i) Let $\mathcal{O}=\mathcal{K}_{\delta} \backslash\left(\Lambda_{\gamma} \times \delta\right)$. Then

$$
\left(e_{\mathcal{O}}, e_{\mathcal{O}}\right)=\left|W^{P}\right| \operatorname{dim}(\delta) \mu_{P l}\left(\hat{\mathfrak{C}}_{\mathcal{O}}\right) .
$$

(ii) These idempotents have the following expansion with respect to any orthonormal basis $B$ of $\mathfrak{H}$ :

$$
e_{\mathcal{O}}=\sum_{b \in B} \chi_{\mathcal{O}}\left(b^{*}\right) b
$$

with

$$
\chi_{\mathcal{O}}(b):=\int_{\pi \in \hat{\mathfrak{C}}_{\mathcal{O}}} \chi_{\pi}(b) d \mu_{P l}(\pi) .
$$

In particular this holds with respect to the orthonormal basis $\left(N_{w}\right)_{w \in W}$.

Proof. (i). The dimension of $\pi \in \hat{\mathfrak{C}}_{\mathcal{O}}$ equals $\left|W^{P}\right| \operatorname{dim}(\delta)$ on an open dense subset, and the measure $\mu_{P l}$ is absolutely continuous with respect to the Haar measure on $\mathcal{O}$. Hence, using the fact that $\mathcal{F}$ is an isometry, we find

$$
\begin{aligned}
\left(e_{\mathcal{O}}, e_{\mathcal{O}}\right) & =\int_{\pi \in \hat{\mathfrak{C}}} \operatorname{Tr}_{V_{\pi}}\left(\mathcal{F}\left(e_{\mathcal{O}}\right)(\pi)\right) d \mu_{P l}(\pi) \\
& =\left|W^{P}\right| \operatorname{dim}(\delta) \mu_{P l}\left(\hat{\mathfrak{C}}_{\mathcal{O}}\right) .
\end{aligned}
$$

(ii). As in (i) we get

$$
\begin{aligned}
\chi_{\mathcal{O}}(b) & =\left(e_{\mathcal{O}}, b\right) \\
& =\int_{\pi \in \hat{\mathfrak{C}}} \operatorname{Tr}_{V_{\pi}}\left(\mathcal{F}\left(e_{\mathcal{O}}\right)(\pi) \mathcal{F}(b)(\pi)\right) d \mu_{P l}(\pi) \\
& =\int_{\pi \in \hat{\mathfrak{C}}_{\mathcal{O}}} \operatorname{Tr}_{V_{\pi}}(\pi(b)) d \mu_{P l}(\pi) \\
& =\int_{\pi \in \hat{\mathfrak{C}}_{\mathcal{O}}} \chi_{\pi}(b) d \mu_{P l}(\pi) .
\end{aligned}
$$

The above depends on the results of [13], but in the special case of isolated points in $\hat{\mathfrak{C}}$ these facts are more elementary: 
ON THE SPECTRAL DECOMPOSITION OF AFFINE HECKE ALGEBRAS 107

Proposition 6.10. When $\pi \in \Delta_{\mathcal{R}}$, put $\mathcal{O}_{\pi}=\{[\pi]\}$ for the corresponding component of $\hat{\mathfrak{C}}$ (an isolated point). Put $e_{\pi}$ for the corresponding central idempotent of $\mathfrak{C}$. The expansion

$$
e_{\pi}=\mu_{P l}(\{\pi\}) \sum_{b \in B} \chi_{\pi}\left(b^{*}\right) b
$$

is convergent in $\mathfrak{S}$.

Proof. The expansion follows as in the above Theorem. It is convergent in $\mathfrak{S}$ because of Corollary 3.33 and Definition 6.6.

Corollary 6.11. Let $B$ be a Hilbert basis of $\mathfrak{H}$. For any residual point $r$ and discrete series representation $\pi \in \Delta_{W_{0} r}$ we have

$$
\sum_{b \in B}\left|\chi_{\pi}(b)\right|^{2}=\frac{\operatorname{dim}(\pi)}{\left|W_{0} r\right| \bar{\kappa}_{W_{0} r} d_{\pi} m_{\{r\}}(r)}
$$

where the constant $\bar{\kappa}_{W_{0} r} \in \mathbb{Q}$ is defined by (3.55), the constant $d_{\pi} \in \mathbb{R}_{+}$ by Definition 3.24 and $m_{\{r\}}(r)$ by Theorem 3.25.

Proof. This follows from $\mu_{P l}(\pi)=\left|W_{0} r\right| \bar{\kappa}_{W_{0} r} d_{\pi} m_{\{r\}}(r)$. Note that $d_{\pi}$ is indeed constant (i.e. independent of $\mathbf{q}$ ) by Theorem 5.6. Also note Conjecture 2.27.

\subsection{Some examples}

6.5.1. The Steinberg representation. A basic example is the Steinberg representation. We obtain a well known expression for the Poincaré series of $W$.

This result was first (for equal labels, using Morse theory) derived by Bott [9], and by elementary means by Steinberg [47]. Macdonald [32] observed that the arbitrary parameter case can be obtained by Steinberg's method. Macdonald proved formula (6.26) below, expressing the Poincaré polynomial in terms of the roots, in an elementary way using case-by-case verifications. In [33] Macdonald reproved the formula in a uniform way. Also note that the Steinberg representation is a representation of $e_{-} \mathcal{H} e_{-}$(cf. 6.3). Hence its formal degree can also be computed by means of the (simpler) techniques of [19].

We assume that $Q \subset X \subset P$. Let $\pi_{S t}$ be the Steinberg representation, which is the representation defined by $\pi_{S t}\left(T_{w}\right)=(-1)^{l(w)}$. This is a one dimensional discrete series representation provided that $r_{S t} \in T_{r s}^{-}$(see Proposition 6.3). Recall that $r_{S t} \in T_{r s}$ is defined by $\forall \alpha \in F_{0}: \alpha\left(r_{S t}\right)=q_{\alpha^{\vee} / 2}^{-1 / 2} q_{\alpha^{\vee}}^{-1}$. Generically this residual point is regular. In this regular case, the residual codimension 1 cosets containing 
$w r_{S t}$ form a normal crossing divisor $D_{w}$ locally at $w r_{S t}$. By Proposition 3.12 we find that $\left[\xi_{w r_{S t}}\right] \in H_{n}\left(U \backslash D_{w}\right)$ (with $U$ a small ball around $w r$ ) is zero if $w \neq e$, and for $w=e$ it is straightforward to see that $\left[\xi_{r_{S t}}\right]=(-1)^{n} . C$, where $C$ is the positive generator of $H_{n}\left(U \backslash D_{e}\right)$. In view of (3.50) we find that (still in the regular case)

$$
\kappa_{w r_{S t}}=\frac{(-1)^{n} \delta_{w, e}}{|X: Q|}
$$

and thus that $\bar{\kappa}_{W_{0} r_{S t}}=(-1)^{n}\left|W_{0}\right|^{-1}|X: Q|^{-1}$.

Hence if $\pi$ is a discrete series representation with central character $r_{S t}$ then, assuming that $r_{S t}$ is regular, equation (3.70) implies that $\pi$ can only have a nonzero weight space for the weight $r_{S t}$. But this weight space is one dimensional, so that $\pi=\pi_{S t}$. Hence in the regular case, the Steinberg representation is the only member of $\Delta_{W_{0} r_{S t}}$, and thus $d_{\pi_{S t}}=1$ in this case.

Inserting the values of these constants, above identity thus specializes to (using the Hilbert bases $\left(N_{w}\right)_{w \in W}$ ) Macdonald's product formula for the Poincaré series of $W$ :

$$
\sum_{w \in W} q(w)^{-1}=\frac{(-1)^{n}|X: Q|}{m_{\left\{r_{S t}\right\}}\left(r_{S t}\right)}
$$

By continuity, this formula holds in general provided that $r_{S t} \in T_{r s}^{-}$. When we take $q(s)=\mathbf{q}$ for all $s \in S^{\text {aff }}$, and $X=Q$, then we obtain

$$
\sum_{w \in W} \mathbf{q}^{-l(w)}=\prod_{i=1}^{n} \frac{\left(\mathbf{q}^{m_{i}+1}-1\right)}{\left(\mathbf{q}^{m_{i}}-1\right)(\mathbf{q}-1)}
$$

where $\left(m_{i}\right)$ is the list of exponents of $W_{0}$.

6.5.2. The subregular unipotent orbit of $S p_{2 n}$. Let $F$ be a nonarchimedean local field, and let $\mathbf{q}$ be the cardinality of its residue field. Consider the group $\mathcal{G}=\mathrm{SO}_{2 n+1}(F)$ for $n \geq 3$. The Langlands dual group of $\mathcal{G}$ is $\hat{G}=\operatorname{Sp}_{2 n}(\mathbb{C})$, whose root datum (with basis) we write as $\mathcal{R}=\left(C_{n}, \mathbb{Z}^{n}, B_{n}, \mathbb{Z}^{n}, F_{0}\right)$, with $F_{0}=\left(e_{1}-e_{2}, \ldots, e_{n-1}-e_{n}, 2 e_{n}\right)$. We normalize the Haar measure of $\mathcal{G}$ by $\operatorname{Vol}(\mathcal{I})=1$, where $\mathcal{I} \subset \mathcal{G}$ is an Iwahori subgroup. Let us compute the formal dimensions of the irreducible square integrable, Iwahori-spherical representations of $\mathcal{G}$ whose Kazhdan-Lusztig parameters $\left(r_{u}, u, \rho\right)$ (cf. Appendix 8) are such that $u$ is the subregular unipotent orbit of $\hat{G}$. Take $r_{u} \in T_{r s}$ dominant in its $W_{0}$-orbit. It follows from the discussion in Appendix 8 that the value $\alpha\left(r_{u}\right)$ with $\alpha \in F_{0}$ is given by $\mathbf{q}^{D_{u}(\alpha) / 2}$, where $D_{u}(\alpha)$ is the weight of $\alpha$ in the Bala-Carter diagram of $u$ (cf. [10]). In our case, the vector of values $\alpha\left(r_{u}\right)$ with $\alpha \in F_{0}$ is $(\mathbf{q}, \ldots, \mathbf{q}, 1, \mathbf{q})$, which is a residual 
point for $\left(\mathcal{R}, q_{1}\right)$, where $q_{1}$ denotes the length multiplicative function $q_{1}(w)=\mathbf{q}^{l(w)}$.

The Springer correspondence for all classical types has been computed explicitly in [44]. We use the description of [30] (see [10]). In our particular case, the partition $\lambda \vdash 2 n$ of elementary divisors of $u$ is $\lambda=(2,2 n-2)$. Thus the Springer representation corresponding with $(u, 1)$ (1 denoting the trivial representation of the component group $A\left(r_{u}, u\right)$ (see Appendix 8)) is the representation $\phi_{(n-1,1)}$ of $W_{0}$ labeled by the double partition $(n-1,1)$ of $n$. This is the reflection representation of $W_{0}$.

The component group is equal to $A\left(r_{u}, u\right) \simeq C_{2}$ (Chapter 13, loc. cit.). Both representations \pm 1 of $A\left(r_{u}, u\right)$ are geometric, and one easily finds that the Springer correspondent of $(u,-1)$ is the representation $\phi_{(-, n)}$ of $W_{0}$. This is the 1-dimensional representation in which $s_{i}$ acts by 1 for $i=1, \ldots, n-1$, and in which $s_{n}$ acts by -1 .

Let us denote by $\pi_{ \pm 1}$ the irreducible square integrable $\mathcal{I}$-spherical representations of $\mathcal{G}$ with the Kazhdan-Lusztig parameters $\left(r_{u}, u, \pm 1\right)$, and put $\rho_{ \pm 1,1}:=\pi_{ \pm 1}^{\mathcal{I}}$. The Kazhdan-Lusztig model [23], and the explicit results of [25] imply the following: $\rho_{1,1}$ is an $(n+1)$-dimensional discrete series representation of $\mathcal{H}\left(\mathcal{R}, q_{1}\right)$, with central character $W_{0} r_{u}$, and with restriction to $\mathcal{H}\left(W_{0}, q_{1}\right)$ whose limit for $\mathbf{q} \rightarrow 1$ is equal to $\phi_{\left(-, 1^{n}\right)} \otimes$ $\left(\phi_{(n-1,1)} \oplus \phi_{(n,-)}\right)$ (here $\phi_{\left(-, 1^{n}\right)}$ is the sign representation, and $\phi_{(n,-)}$ is the trivial representation of $\left.W_{0}\right)$. The representation $\rho_{-1,1}$ is 1 dimensional, and has $\mathcal{H}\left(W_{0}, q_{0}\right)$-type corresponding to $\phi_{\left(-, 1^{n}\right)} \otimes \phi_{(-, n)}=$ $\phi_{\left(1^{n},-\right)}$ in the limit $\mathbf{q} \rightarrow 1$.

According to Corollary 3.32 (also see Subsection 6.1) we have

$$
\operatorname{fdim}\left(\pi_{ \pm 1}\right)=\left|W_{0} r_{u}\right| \bar{\kappa}_{W_{0} r_{u}} d_{\rho_{ \pm 1}} m_{\left\{r_{u}\right\}}\left(r_{u}\right),
$$

with $m_{\left\{r_{u}\right\}}\left(r_{u}\right)$ equal to the rational function $(3.47), \bar{\kappa}_{W_{0} r_{u}} \in \mathbb{Q}^{\times}$and $d_{\rho_{ \pm 1}} \in \mathbb{R}_{+}$, subject to the condition $(n+1) d_{\rho_{+1}}+d_{\rho_{-1}}=1$.

In general I do not know how to compute the constants $\bar{\kappa}_{W_{0} r_{u}}$ and $d_{\rho_{ \pm 1}}$ (there is a tedious "algorithm" for $\bar{\kappa}_{W_{0} r_{u}}$ (analogous to [18]), and for $d_{\rho_{ \pm 1}}$ not even that). However, in the case of regular central characters these constants are easy to determine. In the situation at hand we we are able to determine the constants by slightly deforming $q$, since the orbits of residual points that "emerge" from $r_{u}$ (there are two of them, corresponding to the two representations $\left.\rho_{ \pm 1,1}\right)$ under such a deformation are regular. Moreover, one can show in the current example that the formal dimensions are continuous under this deformation.

So let us consider generic root labels $q_{f}$ (cf. [18], [45]) defined by $q_{f}\left(s_{i}\right)=\mathbf{q}(i=1, \ldots, n-1)$ and $q_{f}\left(s_{n}\right)=\mathbf{q}^{f}$, where $0<f<2$, $f \neq 1$. There are two generic orbits $W_{0} r_{ \pm 1, f}$ of residual points such 
that $W_{0} r_{ \pm 1,1}=W_{0} r_{u}$. By the generic parametrization of [18] of orbits of residual points of the graded affine Hecke algebra (which, by Theorem 7.7, can also be used for $\left(\mathcal{R}, q_{f}\right)$ residual points) of type $C_{n}$ such a generic orbit corresponds to a partition of $n$. In this case the partitions are $\xi_{1}=(n-1,1)$ and $\xi_{-1}=1^{n}$. The (standard basis) coordinates of these residual points (suitably chosen within their $W_{0}$-orbits) are (by [18]) $r_{1, f}=\left(\mathbf{q}^{2-n-f / 2}, \mathbf{q}^{3-n-f / 2}, \ldots, \mathbf{q}^{1-f / 2}\right)$ and $r_{-1, f}=\left(\mathbf{q}^{1-n+f / 2}, \mathbf{q}^{2-n+f / 2}, \ldots, \mathbf{q}^{f / 2}\right)$. In particular, these are regular orbits of residual points.

By Theorem 3.29, for each of these central characters there exists at least 1 irreducible square integrable representation of $\mathcal{H}$. In addition, it is not difficult to see (cf. [45]) that the residual Hilbert algebra of a regular orbit of residual points is in fact simple. Thus for $f \neq 1$, we find precisely two irreducible square integrable representations $\rho_{ \pm 1, f}$, with central characters $W_{0} r_{ \pm 1, f}$.

One checks directly that $r_{-1, f}$ is the $\mathcal{A}$-weight space of a 1 dimensional (square integrable, by Casselman's criterion) representation where $T_{i}(i<n)$ acts by -1 , and $T_{n}$ by $\mathbf{q}^{f}$. This is a continuous family of square integrable representations in the parameter $f$ (if $f$ is in the range $0<f<2$ and $n \geq 3)$. We call this parameter family $\rho_{-1, f}$.

The other orbit $W_{0} r_{1, f}$ also carries a continuous parameter family of square integrable representations $\rho_{1, f}$, the twist by the automorphism $i$ (see Subsection 6.3) of the affine reflection representation of $\mathcal{H}$ (a representation of dimension $(n+1)$ ). To see this, we give the following model for the representation (there are several possible constructions one could invoke here, but none of these is obvious (as far as I know)). Our approach here is based on the simplifying circumstance that the representation contains the sign representation of $\mathcal{H}\left(W_{0}\right)$ (is "anti-spherical").

We will use the spherical function $\phi(\mu, k)$ of the Yang system (cf. [18]), with $R=C_{n}, k_{\alpha}=\log \left(q\left(s_{\alpha}\right)\right)$, and $\mathbf{k}=\log (\mathbf{q})$. Recall that this function depends analytically on $(\mu, k)$. First we consider $-\mathbf{k}<0$ (the attractive case in [18]), and we consider the residual point $\mu=$ $\log \left(w_{0} r_{1, f}\right)=-\mathbf{k}(f / 2+n-2, f / 2+n-3, \ldots, f / 2, f / 2-1)$. The list of positive roots $\alpha$ such that $\alpha(\mu)=-k_{\alpha}$ is $\mathcal{L}=\left(e_{1}-e_{2}, \ldots, e_{n-1}-\right.$ $\left.e_{n}, 2 e_{n-1}\right)$. In order to compute the dimension (in the regular case $f \neq 1$ ) of the graded Hecke module generated by $\phi(\mu, k)$ we have to count the number of exponentials $e^{w \mu}$ which have a nonzero coefficient in $\phi(\mu, k)$. Assuming that $0<f<2, f \neq 1$, we see that $\mu$ satisfies the condition of Lemma 3.3 of [18], and by Remark 3.4 of [18] this shows that $\mu$ is an exponent of $\phi(\mu, k)$. Then $w^{-1} \mu$ is also an exponent 
ON THE SPECTRAL DECOMPOSITION OF AFFINE HECKE ALGEBRAS 111

iff $w \delta=w(n, n-1, \ldots, 1)$ satisfies $w \delta(\alpha)>0$ for all $\alpha \in \mathcal{L}$. One easily verifies that this is satisfied iff $w \delta=(n, n-1, \ldots, 2, \pm 1)$ or $w \delta=(n, n-1, \ldots, \hat{j}, \ldots, 1,-j)(j=n, n-1, \ldots, 2)$. Hence the module generated by $\phi(\mu,-k$ ) (with $\mu$ as above, and $0<f<2$ ) is a spherical discrete series module of the graded Hecke algebra, which is irreducible (this is always true, see the discussion above Section 3, loc. cit.), and of dimension $n+1$ if $f \neq 1$. Now apply the involution $i$ (see Section 5, loc. cit., and also Subsection 6.3) to replace $-\mathbf{k}$ by $\mathbf{k}$, and then integrate the representation (as in [26], Section 9) so obtained to get a representation of $\mathcal{H}$. We obtain a parameter family (depending on $f$ with $0<f<2$ ) of irreducible square integrable representations generated by an anti-spherical vector, of dimension $n+1$ if $f \neq 1$. Now observe that for $f=1$ this representation has to be irreducible of dimension $n+1$ as well, by the classification of the square integrable representations with central character $W_{0} r_{u}$ as described above. We call this $n+1$-dimensional family $\rho_{1, f}$. It follows easily from the above discussion that the characters of $\rho_{ \pm 1, f}$ are continuous in $0<f<2$, and uniformly square integrable.

Hence we can compute the formal dimension of both representations by taking the limit for $f \rightarrow 1$ of the corresponding generic formal dimensions. It is easy to see that

$$
\lim _{f \rightarrow 1} m_{\left\{r_{ \pm 1, f}\right\}}\left(r_{ \pm 1, f}\right)= \pm \frac{1}{2} m_{\left\{r_{u}\right\}}\left(r_{u}\right)
$$

For $f \neq 1$ one obviously has $d_{\rho_{ \pm}}=1$ and $\kappa_{w r_{ \pm 1, f}}= \pm(-1)^{n}|X: Q|^{-1}=$ $\pm(-1)^{n} / 2$ for all $w$ such that $w r_{ \pm 1, f}$ is a weight in $\rho_{ \pm 1, f}$, and $=0$ else.

Combining these facts, we find in the limit $f \rightarrow 1$ that $\left|W_{0} r_{u}\right| \bar{\kappa}_{W_{0} r_{u}}=$ $(-1)^{n}(n+2) / 4$, and $d_{\rho_{ \pm 1}}=1 /(n+2)$. Hence both constants $\lambda_{\rho_{ \pm 1}}$ are equal to $(-1)^{n} / 4$, which is in accordance with Reeder's conjectural formula ([40], equation (0.5)) for the formal dimension (up to a sign). A computation yields:

$$
\operatorname{fdim}\left(\pi_{ \pm 1}\right)=\frac{1}{4} \frac{\mathbf{q}(\mathbf{q}-1)^{n+2}\left(\mathbf{q}^{n-2}-1\right) \prod_{i=1}^{n-2}\left(\mathbf{q}^{2 i+1}-1\right)}{\left(\mathbf{q}^{2}-1\right)\left(\mathbf{q}^{n}-1\right) \prod_{i=1}^{n-1}\left(\mathbf{q}^{2 i}-1\right)}
$$

Remark 6.12. It would be interesting to work out the product formula (3.68) for formal dimensions (without the precise analysis of the constants $\lambda_{\rho}$ ) for classical root systems in general (for "special parameters", see [45]), and to express the answer (in the case of real central characters) in terms of the symbol of the Springer correspondent according to the conjecture in [45]. 


\section{6. $K$-types}

We touch superficially upon the analogue of the problem of the " $K$ type decomposition" of admissible representations of a reductive group for tempered representations of the affine Hecke algebra $\mathcal{H}$. We refer to [40] for a deep connection between the " $K$-types" of an irreducible discrete series representation, and its formal dimension. We refer to [45] for precise conjectures on the $K$-types of the irreducible tempered

modules with real central character for affine Hecke algebras of classical type (and general root labels).

The role of $K$ can be played by any maximal finite type Hecke subalgebra of the form $\mathcal{H}\left(W_{J}\right) \subset \mathcal{H}$, with $J \subset F^{\text {aff }}$ a maximal proper subset. Such a subalgebra is a finite dimensional $*$-subalgebra. The restriction of $\tau$ to $\mathcal{H}\left(W_{J}\right)$ is equal to the usual trace of the finite type Hecke algebra $\mathcal{H}\left(W_{J}\right)$, normalized in such a way that $\tau\left(T_{e}\right)=1$.

For $\sigma \in \hat{W}_{J}$ we denote by $d_{J, \sigma}(q)$ its generic degree with respect to $\mathcal{H}\left(W_{J}\right)$ with label $\left.q\right|_{W_{J}}$. Thus we have (1.1)

$$
\left.\tau\right|_{\mathcal{H}\left(W_{J}\right)}=\left(P_{W_{J}}(q)\right)^{-1} \sum_{\sigma \in \hat{W}_{J}} d_{J, \sigma}(q) \chi_{\sigma},
$$

where $P_{W_{J}}(q)$ denotes the Poincaré polynomial of $W_{J}$ with respect to the label function $q$ (restricted to $W_{J}$ ).

Now observe that the restriction to $\mathcal{H}\left(W_{J}\right)$ of $\pi(\omega)$ is independent of $\omega \in \mathcal{O}$. We denote the multiplicities by $n_{\mathcal{O}}(\sigma)$, thus

$$
\left.\chi_{\pi(\omega)}\right|_{\mathcal{H}\left(W_{J}\right)}=\sum_{\sigma \in \hat{W}_{J}} n_{\mathcal{O}}(\sigma) \chi_{\sigma}
$$

We introduce for $\gamma=\left(\mathcal{R}_{P}, W_{P} r_{P}\right) \in \Gamma$ the following rational functions of $q$ :

$$
M_{\gamma}:=\int_{t \in T_{u}^{P}} m^{P}\left(r_{P} t\right) d^{P} t .
$$

Notice that for all orbits of the form $\mathcal{O}=K_{\delta} \backslash \Lambda_{\gamma} \times \delta$,

$$
\int_{\mathcal{O}} m^{P}(\omega) d^{\mathcal{O}} \omega=M_{\gamma}
$$

From the Plancherel decomposition of $\mathcal{H}$ (Theorem 4.43) we thus obtain the following identities: For all $J \subset F^{\text {aff }}$ a maximal proper subset, and each $\sigma \in \hat{W}_{J}$,

$$
d_{J, \sigma}(q)=P_{W_{J}}(q) \sum_{\gamma \in \Gamma_{a}} M_{\gamma} \sum_{\delta \in \Delta_{\gamma, a}}|W(\mathcal{O})|^{-1} n_{\mathcal{O}}(\sigma) \mu_{\mathcal{R}_{P}, P l}\left(K_{P(\gamma)} \delta\right)
$$


ON THE SPECTRAL DECOMPOSITION OF AFFINE HECKE ALGEBRAS 113

where $\Gamma_{a}$ is a complete set of representatives for the association classes $(=\mathcal{W}$-orbits $)$ in $\Gamma, \Delta_{\gamma, a}$ is a complete set of representatives in $\Delta_{\gamma}$ for the action of $\mathcal{W}(\gamma)$, and where $\mathcal{O}$ denotes the orbit $\mathcal{O}=K_{\delta} \backslash \Lambda_{\gamma} \times \delta$ of cuspidal representations of $\mathcal{H}^{P(\gamma)}$ (for a given pair $(\gamma, \delta) \in \Gamma_{a} \times \Delta_{\gamma, a}$ ).

Example 6.13. It is instructive to verify (6.35) for $R_{0}=B_{2}$ (equal label case), both for $X=Q$ and $X=P$, using the discussion in Example 7.9. The residual point $(\mathbf{q},-1)$ for $X=Q$ (notation of Example 7.9) is the most complicated part. This orbit of residual points carries 2 one-dimensional discrete series representations which are exchanged by the nontrivial affine diagram automorphism. Their direct sum lifts to the two-dimensional irreducible discrete series representation which is carried by the (regular) orbit $\left(\mathbf{q}^{1 / 2},-1\right)$ of residual points for the extended affine Hecke algebra with $X=P$. Using Corollary 6.11 one concludes that the formal dimension of this two-dimensional representation (which is easily computed, since the underlying central character is regular) is equal to the formal dimension of each of the two onedimensional discrete series in which it decomposes upon restriction to the case $X=Q$.

\subsection{A remark on the residual degrees $d_{\pi}$}

We mention one further consequence of Corollary 6.11 regarding the constants $d_{\pi} \in \mathbb{R}_{+}$.

Corollary 6.14. Assume that the constants $f_{s}$ in Convention 2.1 are integers. Let $r$ be a residual point, and let $\pi \in \Delta_{W_{0} r}$. Assume that the character values of $\pi$ on $T_{w}$ are contained in $k\left[\mathbf{q}^{1 / 2}, \mathbf{q}^{-1 / 2}\right]$, where $k$ is a subfield of $\mathbb{C}$. Then $d_{\pi} \in \mathbb{R}_{+} \cap k$.

See also Conjecture 2.27; we expect that the $d_{\pi} \in \mathbb{Q}$.

Proof. The main step is to show that Casselman's bound of Corollary 3.33 becomes uniform in $\mathbf{q}$ under the assumption. Let $r=s c$ and choose $n \in \mathbb{N}$ such that $s^{n}=s$. Let us first fix $\mathbf{q}>1$. Consider the isomorphism of localized Hecke algebras

$$
j_{1 / n}: \mathcal{H}_{q^{n}}^{a n}(U) \rightarrow \mathcal{H}^{a n}\left(U_{1 / n}\right),
$$

where $U=W_{0} s c^{n} \exp B=W_{0} r^{n} \exp B$, with $B$ a suitably small ball around the origin in $\mathfrak{t}_{\mathbb{C}}$. We have, by the assumption that $s^{n}=s$ :

$$
j_{1 / n}\left(\theta_{x, q^{n}}\right)=\theta_{n x} .
$$


On the other hand, for all $s \in F_{0}$, the eigenvalues of the self adjoint operator $\pi\left(j_{1 / n}\left(N_{s, \mathbf{q}^{n}}\right)\right)$ are of the form $\pm \mathbf{q}^{ \pm f_{s} n / 2}$. Hence the operator norms of the operators $\pi\left(j_{1 / n}\left(N_{s, \mathbf{q}^{n}}^{ \pm 1}\right)\right)$ are bounded by $\mathbf{q}^{M n}$, for a suitable constant $M$.

Given $w \in W$ we can write $w=u x v$ with $x \in X^{+}, u \in W^{x}$ and $v \in W_{0}$, where $W^{x}$ denotes the set of shortest length representatives of the left cosets of the stabilizer $W_{x}$ of $x$ in $W_{0}$. If we write $u=s_{i_{1}} \ldots s_{i_{k}}$ and $v=s_{j_{1}} \ldots s_{j_{l}}$, we can thus choose signs $e_{i}$ and $d_{j}$ such that

$$
N_{w}=N_{i_{1}}^{e_{1}} \ldots N_{i_{k}}^{e_{k}} \theta_{x} N_{j_{1}}^{d_{1}} \ldots N_{j_{l}}^{d_{l}} .
$$

Let us simply denote this decomposition by $N_{w}=N_{u}^{e} \theta_{x} N_{v}^{d}$.

Now by Theorem 6.1(ii), and the remark that $j_{1 / n}$ intertwines the action of $\mathcal{H}_{\mathbf{q}^{n}}^{a n}(U)$ on $\left.\pi\right|_{\mathbf{q}^{n}}$ with that of $\mathcal{H}^{a n}\left(U_{1 / n}\right)$ on $\left.\pi\right|_{\mathbf{q}}$, we have $(M$ is a constant, not necessarily the same as above):

$$
\begin{aligned}
\left|\chi_{\pi, \mathbf{q}^{n}}\left(N_{w, \mathbf{q}^{n}}\right)\right|^{2} & =\left|\chi_{\pi, \mathbf{q}}\left(j_{1 / n}\left(N_{w, \mathbf{q}^{n}}\right)\right)\right|^{2} \\
& =\left|\chi_{\pi, \mathbf{q}}\left(j_{1 / n}\left(N_{u, \mathbf{q}^{n}}^{e} N_{v, \mathbf{q}^{n}}^{d}\right) \theta_{n x, \mathbf{q}}\right)\right|^{2} \\
& \leq \operatorname{dim}(\pi)^{2}\left\|\pi\left(j_{1 / n}\left(N_{u, \mathbf{q}^{n}}^{e} N_{v, \mathbf{q}^{n}}^{d}\right)\right)\right\|_{o}^{2}\left\|\theta_{n x, \mathbf{q}}\right\|_{d s}^{2} \\
& \leq C \mathbf{q}^{2 n(M-\epsilon l(x))}
\end{aligned}
$$

where $C$ is independent of $w$ and $n$. In particular, this implies that the highest power of $\mathbf{q}$ in $\left|\chi_{\pi}\left(N_{w}\right)\right|^{2}=\chi_{\pi}\left(N_{w}\right) \chi_{\pi}\left(N_{w^{-1}}\right) \in k_{\mathbb{R}}\left[\mathbf{q}^{1 / 2}, \mathbf{q}^{-1 / 2}\right]$ tends to $-\infty$ with $l(w)$ (with $k_{\mathbb{R}}:=k \cap \mathbb{R}$ ). Hence the left hand side of the equality Corollary 6.11 is a Laurent series in $\mathbf{q}^{-1 / 2}$ with coefficients in $k_{\mathbb{R}}$.

On the other hand, according to Proposition 3.27(iv), $m_{\{r\}}(r)^{-1}$ can be expanded as a Laurent series in $\mathbf{q}^{-1 / 2}$ with coefficients in $\mathbb{Q}$. The desired result follows.

\section{Appendix: Residual Cosets}

\subsection{Introduction and quick guide}

Our approach to the spectral resolution is through residues of certain rational $n$-forms on a complex torus $T$. In order for our method to work well, we need to have a certain a priori knowledge on the geometric and combinatorial properties of the set of poles of these rational forms. The present section serves to collect such facts about the set of poles, and to classify the collection of "residual cosets", the sets of maximal pole order, which will eventually turn out to constitute the projection of support of the Plancherel measure to $W_{0} \backslash T$. 
Recall that we have chosen a rational, positive definite, $W_{0}$-invariant symmetric form on $X$. This defines an isomorphism between $X \otimes_{\mathbb{Z}} \mathbb{Q}$ and $Y \otimes_{\mathbb{Z}} \mathbb{Q}$, and thus also a rational, positive definite symmetric form on $Y$. We extend this form to a positive definite Hermitian form on $\mathfrak{t}_{\mathbb{C}}:=\operatorname{Lie}(T)=Y \otimes_{\mathbb{Z}} \mathbb{C}$, where $T$ is the complex torus $T=\operatorname{Hom}\left(X, \mathbb{C}^{\times}\right)$. Via the exponential covering map exp $: \mathfrak{t}_{\mathbb{C}} \rightarrow T$ this determines a distance function on $T$.

Let $q$ be a set of root labels. If $2 \alpha \notin R_{\mathrm{nr}}$ we formally put $q_{\alpha^{\vee} / 2}=1$, and always $q_{\alpha \vee / 2}^{1 / 2}$ denotes the positive square root of $q_{\alpha^{\vee} / 2}$. Let $L$ be a coset of a subtorus $T^{L} \subset T$ of $T$. Put $R_{L}:=\left\{\alpha \in R_{0} \mid \alpha\left(T^{L}\right)=1\right\}$. This is a parabolic subsystem of $R_{0}$. The corresponding parabolic subgroup of $W_{0}$ is denoted by $W_{L}$. Define

$$
R_{L}^{p}:=\left\{\alpha \in R_{L} \mid \alpha(L)=-q_{\alpha^{\vee} / 2}^{1 / 2} \text { or } \alpha(L)=q_{\alpha^{\vee} / 2}^{1 / 2} q_{\alpha \vee}\right\}
$$

and

$$
R_{L}^{z}:=\left\{\alpha \in R_{L} \mid \alpha(L)= \pm 1\right\} .
$$

We write $R_{L}^{p \text {,ess }}=R_{L}^{p} \backslash R_{L}^{z}$ and $R_{L}^{z \text {,ess }}=R_{L}^{z} \backslash R_{L}^{p}$. We define an index $i_{L}$ by

$$
i_{L}:=\left|R_{L}^{p}\right|-\left|R_{L}^{z}\right|
$$

As a motivation for the somewhat more technical definition in the next subsection, we remark that this index $i_{L}$ computes the order of the pole along $L$ of the rational $(n, 0)$-form

$$
\omega:=\frac{d t}{c(t, q) c\left(t^{-1}, q\right)}
$$

which plays a main role in this paper (cf. equation (3.1)). We will find (cf. Corollary 7.12) that for each coset $L$ of a subtorus of $T$,

$$
i_{L} \leq \operatorname{codim}(L) \text {. }
$$

Suppose that $L$ is a coset such that $i_{L}<\operatorname{codim}(L)$, and let $T_{L}$ denote the subtorus orthogonal to $T^{L}$. Let $C_{L}$ be a cycle of dimension $\operatorname{dim}\left(C_{L}\right)=\operatorname{codim}(L)$ in a sufficiently small neighborhood of $e$ in $T_{L}$, and let $C^{L}$ be any compact cycle in $L \backslash \cup_{L \not \subset L^{\prime}} L^{\prime}$ of dimension $\operatorname{dim}\left(C^{L}\right)=\operatorname{dim}(L)$. Then for every homolorphic function $f$ on $T$,

$$
\int_{C^{L} \times C_{L}} f \omega=0 .
$$

We call a coset $L$ residual if $i_{L}=\operatorname{codim}(L)$. It will turn out that the support of the spectral measure of the restriction of the trace $\tau$ to the center of the Hecke algebra is precisely equal to the union of all 
the "tempered forms" of the residual cosets (see Theorem 3.29). The spectral measure arises as a sum of integrals of the form (7.6).

For technical convenience, the Definition 7.1 of the notion "residual coset" in the next subsection is slightly more complicated. We will define the residual cosets by induction on their codimension in $T$, in such a way that the collection of residual cosets is easily amenable to classification. In the next subsection we discuss their elementary properties and show how the classification can be reduced to the case of residual subspaces in the sense of [18]. These residual subspaces were already classified in the paper [18]. By this classification we verify equation (7.5) (cf. Corollary 7.12). Using Lemma 7.11 this implies that the following are equivalent for a coset $L \subset T$ :

(i) $L$ is residual (in the sense of Definition 7.1).

(ii) $i_{L} \geq \operatorname{codim}(L)$.

(iii) $i_{L}=\operatorname{codim}(L)$.

\subsection{Definition and Classification of Residual Cosets}

We give the following recursive definition of the notion residual coset.

Definition 7.1. A coset $L$ of a subtorus of $T$ is called residual if either $L=T$, or else if there exists a residual coset $M \supset L$ such that $\operatorname{dim}(M)=\operatorname{dim}(L)+1$ and

$$
i_{L} \geq i_{M}+1 \text {. }
$$

Corollary 7.2. The collection of residual cosets is a nonempty, finite collection of cosets of algebraic subtori of $T$, closed for the action of the group of automorphisms of the root system preserving $q$ (in particular the elements of $W_{0}$, but also for example - Id).

Proof. By induction on the codimension. In a residual coset $M$ of codimension $k-1$ we find only finitely many cosets $L \subset M$ of codimension 1 in $M$ with $i_{L}>i_{M}$. The invariance is obvious from the invariance of the index function $i_{L}$.

Proposition 7.3. If $L$ is residual, then

(i) $R_{L}^{\text {pess }}$ spans a subspace $V_{L}$ of dimension $\operatorname{codim}(L)$ in the $\mathbb{Q}$ vectorspace $V=X \otimes \mathbb{Q}$.

(ii) We have $R_{L}=V_{L} \cap R_{0}$, and the rank of $R_{L}$ equals $\operatorname{codim}(L)$.

(iii) Put ${ }_{L} X:=V_{L} \cap X$ and $X^{L}:=X /{ }_{L} X$. Then $T^{L}=\{t \in T \mid$ $\left.x(t)=1 \forall x \in{ }_{L} X\right\}=\operatorname{Hom}\left(X^{L}, \mathbb{C}^{\times}\right)=\left(T^{W_{L}}\right)^{0}$.

(iv) Put $Y_{L}:=Y \cap \mathbb{Q} R_{L}^{\vee}$ and ${ }^{L} X:=Y_{L}^{\perp} \cap X$. Let $X_{L}:=X /{ }^{L} X$. We identify $R_{L}$ with its image in $X_{L}$. Let $F_{L}$ be the basis of 
ON THE SPECTRAL DECOMPOSITION OF AFFINE HECKE ALGEBRAS 117

$R_{L}$ such that $F_{L} \subset R_{0,+}$. Then $\mathcal{R}_{L}:=\left(X_{L}, Y_{L}, R_{L}, R_{L}^{\vee}, F_{L}\right)$ is a root datum.

(v) Put $T_{L}:=\operatorname{Hom}\left(X_{L}, \mathbb{C}^{\times}\right) \subset T$ (we identify $T_{L}$ with its canonical image in $T)$. Then $T_{L}$ is the subtorus in $T$ orthogonal to L. Define $K_{L}:=T^{L} \cap T_{L}=\operatorname{Hom}\left(X /\left({ }_{L} X+{ }^{L} X\right), \mathbb{C}^{\times}\right) \subset T$, a finite subgroup of $T$. The intersection $L \cap T_{L}$ is a $K_{L}$-coset consisting of residual points in $T_{L}$ with respect to the root datum $\mathcal{R}_{L}$ and the root label $q_{L}$ obtained from $q$ by restriction to $R_{L, \mathrm{nr}}^{\vee} \subset R_{\mathrm{nr}}^{\vee}$. When $r_{L} \in T_{L} \cap L$, we have $L=r_{L} T^{L}$. Such $r_{L}$ is determined up to multiplication by elements of $K_{L}$.

Proof. By induction on $\operatorname{codim}(L)$ we may assume that the assertions of (i) and (ii) hold true for $M$ in (7.7). From the definition we see that $R_{L}^{p, e s s} \backslash R_{M}^{p, e s s}$ is not the empty set. An element $\alpha$ of $R_{L}^{p, \text { ess }} \backslash R_{M}^{p, \text { ess }}$ can not be constant on $M$, and hence $\alpha \notin R_{M}=V_{M} \cap R_{0}$. Thus

$$
\operatorname{dim}\left(V_{L}\right) \geq \operatorname{dim}\left(V_{M}\right)+1=\operatorname{codim}(M)+1=\operatorname{codim}(L) .
$$

Since also

$$
V_{L} \subset \operatorname{Lie}\left(T^{L}\right)^{\perp}
$$

equality has to hold. Hence $R_{L} \subset V_{L}$ and $R_{L}$ spans $V_{L}$. Since $R_{L}$ is parabolic, we conclude that $R_{L}=V_{L} \cap R_{0}$. This proves (i) and (ii). The subgroup $\left\{t \in T \mid x(t)=1 \forall x \in{ }_{L} X\right\} \subset T$ is isomorphic to $\operatorname{Hom}\left(X^{L}, \mathbb{C}^{\times}\right)$, which is a torus because $X^{L}$ is free. By (ii) then, its dimension equals $\operatorname{dim}\left(T^{L}\right)$. It contains $T^{L}$, hence is equal to $T^{L}$. It follows that $T^{L}$ is the connected component of the group of fixed points for $W_{L}$, proving (iii). The statements (iv) and (v) are trivial.

For later reference we introduce the following notation. A residual coset $L$ determines a parabolic subsystem $R_{L} \subset R_{0}$, and we associated with this a root datum $\mathcal{R}_{L}$. When $\Sigma \subset R_{0}$ is any root subsystem, not necessarily parabolic, we associate to $\Sigma$ two new root data, namely $\mathcal{R}^{\Sigma}:=\left(X, Y, \Sigma, \Sigma^{\vee}, F_{\Sigma}\right)$ with $F_{\Sigma}$ determined by the requirement $F_{\Sigma} \subset$ $R_{0,+}$, and $\mathcal{R}_{\Sigma}:=\left(X_{\Sigma}, Y_{\Sigma}, \Sigma, \Sigma^{\vee}, F_{\Sigma}\right)$ where the lattice $X \rightarrow X_{\Sigma}$ is the quotient of $X$ by the sublattice orthogonal to $\Sigma^{\vee}$, and $Y_{\Sigma} \subset Y$ is the sublattice of elements of $Y$ which are in the $\mathbb{R}$-linear span of $\Sigma^{\vee}$.

There is an obvious converse to Proposition 7.3:

Proposition 7.4. Let $R^{\prime} \subset R_{0}$ be a parabolic subsystem of roots, and let $T^{L} \subset T$ be the subtorus such that $R^{\prime}=R_{L}$. Let $T_{L} \subset T$ be the subtorus whose Lie algebra $\operatorname{Lie}\left(T_{L}\right)$ is spanned by $R_{L}^{\vee}$. Let $r \in T_{L}$ be a residual point with respect to $\left(\mathcal{R}_{L}, q_{L}\right)$ as in Proposition $7.3(v)$. Then $L:=r T^{L}$ is a residual coset for $(\mathcal{R}, q)$. 
The recursive nature of the definition of residual cosets makes it feasible to give a complete classification of them. By Lemma 7.3, this classification problem reduces to the classification of the residual points. In turn, Lusztig [26] indicates how the classification of residual points reduces to the classification of residual points in the sense of [18] for certain graded affine Hecke algebras. This classification is known by the results in [18]. Let us explain this in detail. Following [26] we call a root datum $\mathcal{R}=\left(X, Y, R_{0}, R_{0}^{\vee}, F_{0}\right)$ primitive if one of the following conditions is satisfied:

(1) $\forall \alpha \in R_{0}: \alpha^{\vee} \notin 2 Y$.

(2) There is a unique $\alpha \in F_{0}$ with $\alpha^{\vee} \in 2 Y$ and $\left\{w(\alpha) \mid w \in W_{0}\right\}$ generates $X$.

A primitive root datum $\mathcal{R}$ satisfying (2) is of the type $C_{n}^{\text {aff }}(n \geq 1)$, by which we mean that

$$
\mathcal{R}=\left(Q\left(B_{n}\right)=\mathbb{Z}^{n}, P\left(C_{n}\right)=\mathbb{Z}^{n}, B_{n}, C_{n},\left\{e_{1}-e_{2}, \ldots, e_{n-1}-e_{n}, e_{n}\right\}\right) .
$$

By [26] we know that every root datum is a direct sum of primitive summands.

Proposition 7.5. Let $r \in T$ be a residual point, and write $r=s c \in$ $T_{u} T_{r s}$ for its polar decomposition (with $T_{u}=\operatorname{Hom}\left(X, S^{1}\right)$ and $T_{r s}=$ $\operatorname{Hom}\left(X, \mathbb{R}_{+}\right)$). The root system

$$
R_{s, 1}:=\left\{\alpha \in R_{1} \mid \alpha(s)=1\right\}
$$

has rank $\operatorname{dim}(T)$. The system

$$
R_{s, 0}:=\left\{\alpha \in R_{0} \mid k \alpha \in R_{s, 1} \text { for some } k \in \mathbb{N}\right\}
$$

contains both $R_{\{r\}}^{p, e s s}$ and $R_{\{r\}}^{z, e s s}$, and $r$ is residual with respect to the affine Hecke subalgebra $\mathcal{H}^{s} \subset \mathcal{H}$ whose root datum is given by $\mathcal{R}^{s}:=$ $\left(X, Y, R_{s, 0}, R_{s, 0}^{\vee}, F_{s, 0}\right)$ (with $F_{s, 0}$ the basis of $R_{s, 0}$ contained in $\left.R_{0,+}\right)$.

Proof. It is clear from the definitions that $R_{s, 0}$ contains $R_{\{r\}}^{p, e s s}$ and $R_{\{r\}}^{z \text {,ess }}$, and hence has maximal rank. Given a full flag of $\mathcal{R}$-residual subspaces $\{c\}=L_{0} \subset L_{1} \subset \cdots \subset L_{n}=T$, satisfying (7.7) at each level, we see that the sets $R_{L_{i}}^{p}, R_{L_{i}}^{z}$ are contained in $R_{s, 0}$. It follows by reverse induction on $i$ (starting with $L_{n}=T$ ) that each element of the flag is $\mathcal{R}^{s}$-residual.

Lemma 7.6. Given a residual point $r=s c$, let $s_{0} \in T_{u}=\operatorname{Hom}\left(X, S^{1}\right)$ be the element which coincides with $s$ on each primitive summand of type $C_{n}^{\text {aff }}$ and is trivial on the complement of these summands. Then $s_{0}$ has at most order 2 . 
ON THE SPECTRAL DECOMPOSITION OF AFFINE HECKE ALGEBRAS 119

Proof. To see this we may assume that $\mathcal{R}$ is of type $C_{n}^{\text {aff }}$. Then $R_{1}$ is of type $C_{n}, s=s_{0}$, and $R_{s, 1}=\left\{\alpha \in R_{1} \mid \alpha\left(s_{0}\right)=1\right\}$, being of maximal rank in $R_{1}$, is of type $C_{k}+C_{n-k}$ for some $k$. In particular, $\pm 2 e_{i} \in R_{s, 1}$ for all $i=1, \ldots, n$. Moreover the index of $\mathbb{Z} R_{s, 1}$ in $\mathbb{Z} R_{1}$ is at most 2 . Thus $s_{0}$ takes values in $\{ \pm 1\}$ on $R_{1}$, and is trivial on elements of the form $\pm 2 e_{i}$. It follows that $s_{0}$ is of order at most 2 on $X=\mathbb{Z}^{n}$.

Denote by $h \in \operatorname{Hom}\left(Q, S^{1}\right)$ the image of $s_{0}$ in $\operatorname{Hom}\left(Q, S^{1}\right)$. Choose root labels $k_{\alpha}=k_{s, \alpha} \in \mathbb{R}$ with $\alpha \in R_{s, 0}$ by the requirement $\left(k_{\alpha}\right.$ depends on the image of $s$ in $\operatorname{Hom}\left(Q, S^{1}\right)$, but we suppress this in the notation if there is no danger of confusion)

$$
\begin{aligned}
\mathrm{e}^{k_{\alpha}} & =q_{\alpha \vee}^{h(\alpha) / 2} q_{\alpha^{\vee}+1}^{1 / 2} \\
& = \begin{cases}q_{\alpha \vee / 2}^{1 / 2} q_{\alpha^{\vee}} & \text { if } h(\alpha)=+1 \\
q_{\alpha \vee / 2}^{1 / 2} & \text { if } h(\alpha)=-1\end{cases}
\end{aligned}
$$

Theorem 7.7. Let $r=s c$ be $a(\mathcal{R}, q)$-residual point. Then $\gamma:=$ $\log (c) \in \mathfrak{t}:=\operatorname{Lie}\left(T_{r s}\right)$ is a residual point in the sense of [18] for the graded Hecke algebra $H^{s}=\mathbb{C}\left[W\left(R_{s, 0}\right)\right] \otimes \operatorname{Sym}(\mathfrak{t})$ with root system $R_{s, 0}$ and root labels $k_{s}:=\left(k_{s, \alpha}\right)_{\alpha \in R_{s, 0}}$. This means explicitly that there exists a full flag of affine linear subspaces $\{\gamma\}=\mathfrak{l}_{n} \subset \mathfrak{l}_{n-1} \subset \cdots \subset \mathfrak{l}_{0}=\mathfrak{t}$ such that the sequence

$$
i_{s, \mathfrak{l}_{i}}:=\left|R_{s, 0, i}^{p}\right|-\left|R_{s, 0, i}^{z}\right|
$$

is strictly increasing, where

$$
R_{s, 0, i}^{p}=\left\{\alpha \in R_{s, 0} \mid \alpha\left(\mathfrak{l}_{i}\right)=k_{s, \alpha}\right\},
$$

and

$$
R_{s, 0, i}^{z}=\left\{\alpha \in R_{s, 0} \mid \alpha\left(\mathfrak{l}_{i}\right)=0\right\} .
$$

Conversely, given a $s \in T_{u}$ such that $R_{s, 1} \subset R_{1}$ has rank equal to $\operatorname{rank}(X)$, and a residual point $\gamma \in \mathfrak{t}$ for the root system $R_{s, 0}$ with labels $\left(k_{s, \alpha}\right)$ defined by (7.8), the point $r:=s \exp \gamma$ is $(\mathcal{R}, q)$-residual. This sets up a $1-1$ correspondence between $W_{0}$-orbits of $(\mathcal{R}, q)$ - residual points and the collection of pairs $(s, \gamma)$ where $s$ runs over the $W_{0}$-orbits of elements of $T_{u}$ such that $R_{s, 1}$ has rank equal to $\operatorname{rank}(X)$, and $\gamma \in \mathfrak{t}$ runs over the $W\left(R_{s, 0}\right)$-orbits of residual points (in the sense of [18]) for $R_{s, 0}$ with the labels $k_{s}$.

Proof. Straightforward from the definitions.

For convenience we include the following lemma: 
Lemma 7.8. If the rank of $R_{0}$ equals the rank of $X$ (a necessary condition for existence of residual points!), the $W_{0}$-orbits of points $s \in T_{u}$ such that $R_{s, 1} \subset R_{1}$ has maximal rank correspond $1-1$ to the $\operatorname{Hom}\left(P\left(R_{1}\right) / X, S^{1}\right) \simeq Y / Q\left(R_{1}^{\vee}\right)$-orbits on the affine Dynkin diagram $R_{1}^{(1)}$. In particular, $R_{s, 1}$ only depends on the corresponding $P\left(R_{1}^{\vee}\right) / Q\left(R_{1}^{\vee}\right)$-orbit of vertices of $R_{1}^{(1)}$.

Proof. In the compact torus $\operatorname{Hom}\left(P\left(R_{1}\right), S^{1}\right)$, the $W_{0}$-orbits of such points correspond to the vertices of the fundamental alcove for the action of the affine Weyl group $W_{0} \ltimes 2 \pi i Q\left(R_{1}^{\vee}\right)$ on $Y \otimes 2 \pi i \mathbb{R}$. Now we have to restrict to $X \subset P\left(R_{1}\right)$.

With the results of this subsection at hand, the classification of residual cosets is now reduced to the classification of residual subspaces as was given in [18].

Example 7.9. Let $R_{0}=B_{2}=\left\{ \pm e_{1}, \pm e_{2}, \pm e_{1} \pm e_{2}\right\}$ with basis $\alpha_{1}=$ $e_{1}-e_{2}, \alpha_{2}=e_{2}$, and let $X=Q=\mathbb{Z}^{2}$ (this is $C_{2}^{\text {aff }}$ ). Assume that $q\left(s_{i}\right)=\mathbf{q}$ for $i=0,1,2$. Then $R_{1}=\left\{ \pm 2 e_{1}, \pm 2 e_{2}, \pm e_{1} \pm e_{2}\right\}$ and thus $X=P\left(R_{1}\right)$. We use $\left(\alpha_{1}, \alpha_{2}\right)$ as a basis of $X$ (so a point $t \in T$ is represented by $\left.\left(\alpha_{1}(t), \alpha_{2}(t)\right)\right)$. The orbits of points $s \in T_{u}$ such that $R_{s, 1}$ has rank 2 are represented by $(1,1),(1,-1)$ and $(-1,1)$. The latter point corresponds to $R_{s, 0}=\left\{ \pm e_{1}, \pm e_{2}\right\}$, but since it has value -1 on $\pm e_{1}$, the labels of $\pm e_{1}$ are equal to 1 (by (7.8)). Therefore there are no residual points associated with $(-1,1)$. The other two points each give one orbit of residual points, namely $(\mathbf{q}, \mathbf{q})$ and $(\mathbf{q},-1)$.

In addition we have 2 orbits of one-dimensional residual cosets (with $K_{\{1\}}=C_{2}$ and $\left.K_{\{2\}}=1\right)$, and finally the principal two-dimensional one, $T$.

Let us now consider $R_{0}=B_{2}$ with the lattice $X=P$ and again $q\left(s_{i}\right)=\mathbf{q}$ for $i=0,1,2$. We take $\left(\alpha_{1} / 2, \alpha_{2}\right)$ as a basis for $P$. Now $R_{1}=R_{0}$, and thus $X=P\left(R_{1}\right)$. So again we have 3 orbits of points $s$ for which the rank of $R_{s, 0}$ is 2 , namely $(1,1),(1,-1)$ and $(-1,1)$. Each corresponds to a (regular) orbit of residual points: $\left(\mathbf{q}^{1 / 2}, \mathbf{q}\right),\left(\mathbf{q}^{1 / 2},-1\right)$, and $\left(-\mathbf{q}^{1 / 2}, \mathbf{q}\right)$.

In addition there are 3 one-dimensional residual cosets, 2 associated with $P=\{1\}$ (with $K_{\{1\}}=1$ ) and 1 with $P=\{2\} \quad$ with $K_{\{2\}}=C_{2}$ ). Finally we have the principal residual coset $T$.

\subsection{Properties of residual and tempered cosets}

In the derivation of the Plancherel formula of the affine Hecke algebra, the following properties of residual cosets will play a crucial role. 
ON THE SPECTRAL DECOMPOSITION OF AFFINE HECKE ALGEBRAS 121

Theorem 7.10. For each residual coset $L \subset T$ we have

$$
i_{L}=\operatorname{codim}(L) .
$$

In other words, for every inclusion $L \subset M$ of residual cosets with $\operatorname{dim}(L)=\operatorname{dim}(M)-1$, the inequality (7.7) is actually an equality.

Proof. Unfortunately, I have no classification free proof of this fact. With the classification of residual subspaces at hand it can be checked on a case-by-case basis. By the previous subsection (Proposition 7.3 and Theorem 7.7) the verification reduces to the case of residual points for graded affine Hecke algebras. In [18] (cf. Theorem 3.9) this matter was verified.

Theorem 7.10 has important consequences, as we will see later. At this point we show that the definition of residual cosets can be simplified as a consequence of Theorem 7.10. We begin with a simple lemma:

Lemma 7.11. Let $V$ be a complex vector space of dimension $n$, and suppose that $\mathcal{L}$ is the intersection lattice of a set $\mathcal{P}$ of linear hyperplanes in $V$. Assume that each hyperplane $H \in \mathcal{P}$ comes with a multiplicity $m_{H} \in \mathbb{Z}$, and define the multiplicity $m_{L}$ for $L \in \mathcal{L}$ by $m_{L}:=\sum m_{H}$, where the sum is taken over the hyperplanes $H \in \mathcal{P}$ such that $L \subset H$. Assume that $\{0\} \in \mathcal{L}$ and that $m_{\{0\}} \geq n$. Then there exists a full flag of subspaces $V=V_{0} \supset V_{1} \cdots \supset V_{n}=\{0\}$ such that $m_{k}:=m_{V_{k}} \geq k$.

Proof. We construct the sequence inductively, starting with $V_{0}$. Suppose we already constructed the flag up to $V_{k}$, with $k \leq n-2$. Let $\mathcal{P}_{k} \subset \mathcal{L}$ denote the set of elements of $\mathcal{L}$ of dimension $n-k-1$ contained in $V_{k}$, and let $N_{k}$ denote the cardinality of $\mathcal{P}_{k}$. By assumption, $N_{k} \geq n-k \geq 2$. Since every $H \in \mathcal{P}$ either contains $V_{k}$ or intersects $V_{k}$ in an element of $\mathcal{P}_{k}$, we have

$$
\sum_{L \in \mathcal{P}_{k}}\left(m_{L}-m_{k}\right)=m_{n}-m_{k}
$$

Assume that $\forall L \in \mathcal{P}_{k}: m_{L} \leq k$. Then, because $m_{k} \geq k$ and $N_{k} \geq 2$,

$$
m_{n} \leq k N_{k}+\left(1-N_{k}\right) m_{k} \leq k \leq n-2,
$$

contradicting the assumption $m_{n} \geq n$. Hence there exists a $L \in \mathcal{P}_{k}$ with $m_{L} \geq k+1$, which we can define to be $V_{k+1}$.

Corollary 7.12. For every coset $L \subset T$ one has $i_{L} \leq \operatorname{codim}(L)$, and $L$ is residual if and only if $i_{L}=\operatorname{codim}(L)$. 
Proof. Define $\mathcal{P}$ to be the (multi-)set of codimension 1 cosets of $T$ arising as connected components of the following codimension 1 sets:

$$
\begin{aligned}
& L_{\alpha, 1}^{+}:=\left\{t \in T \mid \alpha(t)=q_{\alpha \vee} q_{\alpha \vee / 2}^{1 / 2}\right\} \\
& L_{\alpha, 2}^{+}:=\left\{t \in T \mid \alpha(t)=-q_{\alpha \vee / 2}^{1 / 2}\right\} \\
& L_{\alpha, 1}^{-}:=\{t \in T \mid \alpha(t)=1\} \\
& L_{\alpha, 2}^{-}:=\{t \in T \mid \alpha(t)=-1\}
\end{aligned}
$$

Here $\alpha \in R_{0}$, and $q_{\alpha^{\vee} / 2}=1$ when $2 \alpha \notin R_{1}$. We give the components of $L_{\alpha, 1}^{+}, L_{\alpha, 1}^{+}$the index +1 , and we give the components of $L_{\alpha, 1}^{-}, L_{\alpha, 1}^{-}$ index -1 .

Suppose that $L$ is any coset of a subtorus $T^{L}$ in $T$. Then $i_{L}$ is equal to the sum of the indices the elements of $\mathcal{P}$ containing $L$.

Assume that $i_{L} \geq \operatorname{codim}(L)=k$. By Lemma 7.11 there exists a sequence $L \subset L_{k-e} \subset L_{k-e-1} \cdots \subset L_{0}=T$ of components of intersections of elements of $\mathcal{P}$ such that $i_{L_{k-e}}=i_{L} \geq k$ and $i_{L_{j}} \geq j=\operatorname{codim}\left(L_{j}\right)$ (we did not assume that $L$ is a component of an intersection of elements in the multiset $\mathcal{P}$, hence $e>0$ may occur). If $k(0)$ is the smallest index such that $i_{L_{k(0)}}>k(0)$, then $L_{k(0)}$ is by definition residual, and thus violates Theorem 7.10. Hence such $k(0)$ does not exist and we conclude that $i_{L_{k}}=k$ for all $k$. This proves that $e=0$ and that $L$ is residual.

Remark 7.13. This solves the question raised in Remark 3.11 of [18].

Theorem 7.14. $\quad$ (i) Let $R_{0}$ be indecomposable, and let $r=c$ be a real residual point in $\overline{T_{r s,+}}$. If $\omega: T_{r s} \rightarrow T_{r s}$ is a homomorphism which acts on the root system $R_{0}$ by means of a diagram automorphism of $F_{0}$, then $\omega(r)=r$.

(ii) Define * $: T \rightarrow T$ by $x\left(t^{*}\right)=\overline{x(t)^{-1}}$. If $r=c s \in T$ is a residual point, then $r^{*} \in W\left(R_{s, 0}\right) r$.

(iii) If $r=s c$ is a residual point, then the values $\alpha(c)$ of the roots $\alpha \in R_{0}$ on c are in the subgroup of $\mathbb{R}_{+}$generated by the positive square roots of the root labels $q_{\alpha} \vee$ with $\alpha \in R_{n r}$.

Proof. (i). If $R_{0}$ allows a nontrivial diagram automorphism then $R_{0}$ is simply laced. So we are in the situation of the Bala-Carter classification of distinguished weighted Dynkin diagrams. A glance at the tables of section 5.9 of [10] shows that this fact holds true.

(ii). This is a consequence of (i), since $*: s T_{r s} \rightarrow s T_{r s}$ acts on $R_{s, 0}$ by means of an automorphism (see also [18], Theorem 3.10) which acts trivially on the set of indecomposable summands of $R_{s, 0}$.

(iii). For this fact I have also no other proof to offer than a caseby-case checking, using the results of this section and the list of real 
residual points from [18]. The amount of work reduces a lot by the remark that it is well known in the simply laced cases (see Corollary 8.2 of the appendix Section 8).

In the classical cases other than $C_{n}^{\text {aff }}$, it follows from a well known theorem of Borel and de Siebenthal [8] that the index of $Q\left(R_{s, 0}\right) \subset Q$ is at most 2. Hence the desired result follows if we verify that for real residual points of the classical root systems, the values $\alpha(c)$ are in the subgroup of $\mathbb{R}_{+}$generated by the root labels, which is direct from the classification lists in [18].

For the real points of $C_{n}^{\text {aff }}$ it is also immediate from the above and (7.8). For nonreal points $r=s c$ we look at (the proof of) Lemma 7.6. If $s$ has order 2 , then $R_{s, 1}$ is of type $C_{k}+C_{n-k}$. We need to check the values of the roots $e_{n}$ and $e_{k}-e_{k+1}$ on $c$ in this case. But the roots $2 e_{i}$ are in $R_{s, 1}$, and take rational values in the labels $q_{\alpha^{\vee}}\left(\alpha \in R_{n r}\right)$ on $c$.

The real residual points of $F_{4}$ are all rational in the root labels (see [18]). Again using the Theorem of Borel and de Siebenthal, we need to check in addition the nonreal residual points $r=s c$ with $R_{s, 0}=$ $A_{2} \times A_{2} \subset F_{4}$ (generating a lattice of index 3 in $Q\left(F_{4}\right)$ ) and $R_{s, 0}=$ $A_{3} \times A_{1} \subset F_{4}$ (index 4$)$. These cases can be checked without difficulty.

In the case of $G_{2}$, there are generically 3 real residual points, two of which have rational coordinates and one has rational coordinates only in the square roots of the labels. In addition there are two nonreal residual points $r=s c$ for $G_{2}$, which are easily checked. (We need to check only the case with $R_{s, 0}=A_{2}$ (index 3 in $\left.Q\left(G_{2}\right)\right)$ ).

Remark 7.15. In fact the result (ii) of the previous Theorem will also turn out to be a consequence of Theorem 3.29, in view of Proposition 2.13.

Definition 7.16. Let $L$ be a residual coset, and write $L=r_{L} T^{L}$ with $r_{L} \in T_{L} \cap L$. This is determined up to multiplication of $r_{L}$ by elements of the finite group $K_{L}=T_{L} \cap T^{L}$. Write $r_{L}=s_{L} c_{L}$ with $s_{L} \in T_{L, u}$ and $c_{L} \in T_{L, r s}$. We call $c_{L}$ the "center" of $L$, and we call $L^{\text {temp }}:=r_{L} T_{u}^{L}$ the tempered compact form of $L$ (both notions are independent of the choice of $r_{L}$, since $\left.K_{L} \subset T_{u}^{L}\right)$. The cosets of the form $L^{\text {temp }}$ in $T$ will be called "tempered residual cosets".

Theorem 7.17. Suppose that $L \subset M$ are two residual cosets. Write $L=r_{L} T^{L}=s_{L} c_{L} T^{L}$ and $M=r_{M} T^{M}=s_{M} c_{M} T^{M}$ as before. If $c_{L}=c_{M}$ then $L=M$.

Proof. According to Proposition 3.11, $c_{L}=c_{M} \Leftrightarrow e \in c_{L}^{-1} T_{M, r s}:=$ $M_{L} \Leftrightarrow L^{t e m p} \subset M^{t e m p}$. Hence the proof reduces to Remark 3.14 of [18], 
or can be proved directly in our setup in the same way, cf. Remark 3.26 .

Theorem 7.17 shows that a tempered coset can not be a subset of a strictly larger tempered coset. In fact even more is true:

Theorem 7.18. (Slooten [46] (cf. [45] for the classical cases)) Let $L_{1}$ and $L_{2}$ be residual subspaces. If $L_{1}^{\text {temp }} \cap L_{2}^{\text {temp }} \neq \emptyset$ then $L_{1}=w\left(L_{2}\right)$ for some $w \in W_{0}$.

We will not use this result in this paper, but it is important for the combinatorial fine structure of the spectrum of $\mathfrak{C}$. We note that the proof of this statement reduces easily to the case of two residual subspaces (in the sense of [18]) with the same center. This reduces the statement of the theorem to the problem in Remark 3.12 of [18]. This problem was solved by Slooten [46].

\section{Appendix: Kazhdan-Lusztig parameters}

Let $F$ be a p-adic field. Let $\mathcal{G}$ be the group of $F$-rational points of a split semisimple algebraic group of adjoint type over $F$, and let $\mathcal{I}$ be an Iwahori subgroup of $\mathcal{G}$. The centralizer algebra of the representation of $\mathcal{G}$ induced from the trivial representation of $\mathcal{I}$ is isomorphic to an affine Hecke algebra $\mathcal{H}$ with "equal labels", that is, the labels are given as in Convention 2.1 with q equal to the cardinality of the residue field of $F$, and the exponents $f_{s}$ all equal to 1 . Moreover, the lattice $X$ is equal to the weight lattice of $R_{0}$ in this case. The Langlands dual group $G$ is the simply connected semisimple group with root system $R_{0}$, and the torus $T$ can be viewed as a maximal torus in $G$.

In this situation Kazhdan and Lusztig [23] have given a complete classification of the irreducible representations of $\mathcal{H}$, and also of the tempered and square integrable irreducible representations. Let us explain the connection with residual cosets explicitly.

We assume that we are in the "equal label case" in this subsection, unless stated otherwise. We put $k=\log (\mathbf{q}) / 2$. Let $G$ be a connected semisimple group over $\mathbb{C}$, with fixed maximal torus $T=\operatorname{Hom}\left(X, \mathbb{C}^{\times}\right)$. We make no assumption on the isogeny class of $G$ yet.

Proposition 8.1. $\quad$ (i) If $r$ is a residual point with polar decomposition $r=s c=s \exp (\gamma) \in T_{u} T_{r s}$ and $\gamma$ dominant, then the centralizer $C_{\mathfrak{g}}(s)$ of $s$ in $\mathfrak{g}:=\operatorname{Lie}(G)$ is a semisimple subalgebra of $\mathfrak{g}$ of rank equal to $\mathrm{rk}(\mathfrak{g})$, and $\gamma / k$ is the weighted Dynkin diagram (cf. [10]) of a distinguished nilpotent class of $C_{\mathfrak{g}}(s)$. 
ON THE SPECTRAL DECOMPOSITION OF AFFINE HECKE ALGEBRAS 125

(ii) Conversely, let $s \in T_{u}$ be such that the centralizer algebra $C_{\mathfrak{g}}(s)$ is semisimple and let $e \in C_{\mathfrak{g}}(s)$ be a distinguished nilpotent element. If $h$ denotes the weighted Dynkin diagram of $e$ then $r=s c$ with $c:=\exp (k h)$ is a residual point.

(iii) The above maps define a $1-1$ correspondence between $W_{0^{-}}$ orbits of residual points on the one hand, and conjugacy classes of pairs $(s, e)$ with $s \in G$ semisimple such that $C_{\mathfrak{g}}(s)$ is semisimple, and e a distinguished nilpotent element in $C_{\mathfrak{g}}(s)$.

(iv) Likewise there is a $1-1$ correspondence between $W_{0}$-orbits of residual points and conjugacy classes of pairs $(s, u)$ with $C_{G}(s)$ semisimple and $u$ a distinguished unipotent element of $C_{G}(s)^{0}$.

Proof. (i). We already saw in Appendix 7 that the rank of $C_{\mathfrak{g}}(s)$ is indeed maximal. So we are reduced to the case $s=1$. Let $\langle\mathbf{q}\rangle$ denote the group of integer powers of $\mathbf{q}$, and denote by $R_{\mathbf{q}} \subset R_{0}$ the root subsystem of roots $\alpha \in R_{0}$ such that $\alpha(c) \in\langle\mathbf{q}\rangle$. Now $R_{\mathbf{q}}$ is a root subsystem of rank equal to $\operatorname{rk}\left(R_{0}\right)$, with the property that $\forall \alpha, \beta \in R_{\mathbf{q}}$ such that $\alpha+\beta \in R_{0}$ we have $\alpha+\beta \in R_{\mathbf{q}}$. Of course, $c$ is a residual point of $R_{\mathbf{q}}$. By an elementary result of Borel and De Siebenthal there exists a finite subgroup $Z \subset T_{u}$ such that $C_{\mathfrak{g}}(Z)$ is semisimple with root system $R_{\mathbf{q}}$.

We claim that for every simple root $\alpha$ of $R_{\mathbf{q}}$ we have $\alpha(c)=1$ or $\alpha(c)=\mathbf{q}$. To see this, observe that all the roots $\alpha \in R_{\mathbf{q}}$ with $\alpha(c)=\mathbf{q}$ are in the parabolic system obtained from $R_{\mathbf{q}}$ by omitting the simple roots $\alpha$ such that $\alpha(c)=\mathbf{q}^{l}$ with $l>1$. If this would be a proper parabolic subsystem, $c$ would violate Theorem 7.10 in this parabolic. This proves the claim.

Define the element $h:=\gamma / k$. Note that $h$ belongs to $2 P\left(R_{\mathbf{q}}^{\vee}\right)$ by the previous remarks. Consider the grading of $R_{\mathbf{q}}$ given by this element, and define a standard parabolic subalgebra $\mathfrak{p}$ of $C_{\mathfrak{g}}(Z)$ by

$$
\mathfrak{p}:=\mathfrak{t} \oplus \sum_{\left\{\alpha \in R_{\mathbf{q}}: \alpha(h) \geq 0\right\}} \mathfrak{g}_{\alpha}=\sum_{i \geq 0} C_{\mathfrak{g}}(Z)(i) .
$$

Its nilpotent radical $\mathfrak{n}$ is

$$
\mathfrak{n}:=\sum_{i \geq 2} C_{\mathfrak{g}}(Z)(i)
$$

and by the definition of residual points we see that $P \subset C_{\mathfrak{g}}(Z)$ is a distinguished parabolic subalgebra (see [10], Corollary 5.8.3.). According to ([10], Proposition 5.8.8.) we can choose $e \in \mathfrak{n}(2)$ in the Richardson class associated with $\mathfrak{p}$, and $f \in C_{\mathfrak{g}}(Z)(-2)$, such that $(f, h, e)$ form a $\mathfrak{s l}_{2}$-triple in $C_{\mathfrak{g}}(Z)$. By $\mathfrak{s l}_{2}$ representation theory it is now clear that 
$h \in P\left(R_{0}^{\vee}\right)$. Consider the grading of $\mathfrak{g}$ and $R_{0}$ induced by $h$. By definition of $Z$ we see that $\mathfrak{g}(0)=C_{\mathfrak{g}}(Z)(0)$ and $\mathfrak{g}(2)=C_{\mathfrak{g}}(Z)(2)$. Hence $e$ is distinguished in $\mathfrak{g}$ by ([10], Proposition 5.7.5.), proving the desired result. Note also that, by ([10], Proposition 5.7.6.), in fact $\mathfrak{g}(1)=0$, and hence that $R_{\mathbf{q}}=R_{0}$.

(ii). Is immediate from the defining property

$$
\operatorname{dim}\left(C_{\mathfrak{g}}(s)(0)\right)=\operatorname{dim}\left(C_{\mathfrak{g}}(s)(2)\right)
$$

of the grading with respect to the Dynkin diagram of a distinguished class.

(iii). Is clear by the well known $1-1$ correspondence between distinguished classes and their Dynkin diagrams.

(iv). The result follows from the well known $1-1$ correspondence between unipotent classes and nilpotent classes for connected semisimple groups over $\mathbb{C}$.

Corollary 8.2. From the proof of Proposition 8.1(i) we see that if $r=s c$ is a residual point, then $\alpha(c) \in\left\langle\mathbf{q}^{1 / 2}\right\rangle$ for all $\alpha \in R_{0}$. If $s=1$ we have $\alpha(c) \in\langle\mathbf{q}\rangle$ for all $\alpha \in R_{0}$.

Let $M \subset T$ be a residual coset. Write $M=r T^{M} \subset T \subset G$ with $r \in T_{M}$ as in Proposition 7.3. Let $r=s c=s \exp (k h / 2)$ be the polar decomposition of $r$ in $T_{M}$. Let $L_{M} \subset G$ be the Levi subgroup $L_{M}:=$ $C_{G}\left(T^{M}\right)$ and let $L_{M}^{\prime}$ denote its semisimple part. By Proposition 7.3 we see that the root system of $L_{M}^{\prime}$ is $R_{M}, T_{M}$ is a maximal torus of $L_{M}^{\prime}$, and the connected center of $L_{M}$ is $T^{M}$. Moreover, $r \in T_{M}$ is a residual point with respect to $R_{M}$. Thus by Proposition 8.1, $C_{L_{M}^{\prime}}(s)$ is semisimple, and there exists a distinguished unipotent element $u=$ $\exp (e)$ in $C_{L_{M}^{\prime}}(s)^{0}$ such that $[h, e]=2 e$. This implies that the set $N=N_{u}$ of all elements $t \in G$ such that

$$
t u t^{-1}=u^{\mathbf{q}} \text {. }
$$

is of the form $N=r C_{G}(u)$. The centralizer $C_{G}(r, u)=C_{G}(s, c, u)$ is known to be maximal reductive in $C_{G}(s, u)$, and it contains $T^{M}$. Its intersection with $L_{M}^{\prime}$ is also reductive but, since $u$ is distinguished in $C_{L_{M}^{\prime}}(s)^{0}$, the rank of this intersection is 0 . Hence $L_{M}^{\prime} \cap C_{G}(r, u)$ is finite. We conclude that $T^{M}$ is a maximal torus in $C_{G}(s, u)$. Let $u^{\prime}$ be another unipotent element in $G$ such that $M \subset N^{\prime}=N_{u^{\prime}}$ and such that $T^{M}$ is a maximal torus of $C_{G}\left(s, u^{\prime}\right)$. We see that $u^{\prime} \in C_{L_{M}^{\prime}}(s)^{0}$ is distinguished and associated to the Dynkin diagram $h$. Hence $u^{\prime}$ is conjugate to $u$ in $C_{L_{M}^{\prime}}(s)^{0}$ by an element of $C_{L_{M}^{\prime}}(r)$. We have shown: 
Proposition 8.3. For each residual coset $M=r T^{M}=s c T^{M} \subset T$ there exists a unipotent element $u$ such that tut ${ }^{-1}=u^{\mathbf{q}}$ for all $t \in M$, and such that $T^{M}$ is a maximal torus of $C_{G}(s, u)$. This $u$ is an element of $C_{L_{M}^{\prime}}(s)$ with $L_{M}:=C_{G}\left(T^{M}\right)$, and is distinguished in this semisimple group. It is unique up to conjugation by elements of the reductive group $C_{L_{M}^{\prime}}(r)$.

Let us consider the converse construction. From now in this subsection we assume that $G$ is simply connected. We will be interested in conjugacy classes of pairs $(t, u)$ with $t$ semisimple and $u$ unipotent, satisfying (8.1). We choose an element $(t, u)$ in the conjugacy class. By Jacobson-Morozov's theorem there exists a homomorphism

$$
\phi: S L_{2}(\mathbb{C}) \mapsto G
$$

such that

$$
u=\phi\left(\begin{array}{ll}
1 & 1 \\
0 & 1
\end{array}\right)
$$

We put

$$
c:=\phi\left(\begin{array}{cc}
\mathbf{q}^{1 / 2} & 0 \\
0 & \mathbf{q}^{-1 / 2}
\end{array}\right), h:=d \phi\left(\begin{array}{cc}
1 & 0 \\
0 & -1
\end{array}\right), e:=d \phi\left(\begin{array}{ll}
0 & 1 \\
0 & 0
\end{array}\right) .
$$

Denote by $C_{G}(\phi)$ the centralizer of the image of $\phi$. We have $C_{G}(\phi)=$ $C_{G}(d \phi)$, and by $\mathfrak{s l}_{2}$ representation theory we see that $C_{G}(d \phi)=C_{G}(h, e)$. Hence $C_{G}(\phi)=C_{G}(c, u)$. By [23], Section 2, this is a maximal reductive subgroup of $C_{G}(u)$, and we can choose $\phi$ in such a way that $t \in c C_{G}(\phi)$. In this case $t$ commutes with $c$, and thus $t_{1}:=t c^{-1} \in C_{G}(\phi)$ commutes with $c, t$, and is semisimple. It follows that $C_{G}\left(t_{1}, \phi\right)=C_{G}(\phi) \cap C_{G}\left(t_{1}\right)$ is reductive in $C_{G}\left(t_{1}\right)$, and contains $t_{1}$ in its center. According to [23], the choice of $\phi$ such that $t_{1} \in C_{G}(\phi)$ is unique up to conjugation by elements in $C_{G}(t, u)$.

By conjugating $(t, u)$ and $\phi$ suitably we can arrange that $\bar{T}:=(T \cap$ $\left.C_{G}\left(t_{1}, \phi\right)\right)^{0}$ is a maximal torus of $C_{G}\left(t_{1}, \phi\right)$. Put $L=C_{G}(\bar{T})$, a Levi group of $G$. We claim that $L$ is minimal among the Levi groups of $G$ containing $\phi$ and $t_{1}$. Indeed, if $N$ would be a strictly smaller Levi group of $G$ also containing $\phi$ and $t_{1}$, then its connected center $T^{N}$ would be a torus contained in $C_{G}\left(t_{1}, \phi\right)$ on the one hand, but strictly larger than $\bar{T}$ on the other hand. This contradicts the choice of $\bar{T}$, proving the claim. In particular, since the connected center $T^{L}$ of $L$ satisfies $\bar{T} \subset T^{L} \subset C_{G}\left(t_{1}, \phi\right)$, we have the equality $\bar{T}=T^{L}$.

Note that maximal tori of $L$ are the maximal tori of $G$ containing $T^{L}$, and these are conjugate under the action of $L$. The derived group $L^{\prime}$ is simply connected, because the cocharacter lattice $Y_{L}$ of its torus $T_{L}$ equals $Y_{L}=Q\left(R_{0}^{\vee}\right) \cap \mathbb{Q} R_{L}^{\vee}=Q\left(R_{L}^{\vee}\right)$. Hence, by a well known 
result of Steinberg, $C:=C_{L}\left(t_{1}\right) \subset L$ is connected, and reductive. This implies that there exist maximal tori of $C$ containing $t$. Thus there exist maximal tori of $L$ containing both the commuting semisimple elements $t_{1}$ and $t$. Therefore we may and will assume (after conjugation of $(t, u)$ and $\phi$ by a suitable element of $L$ ) that $T^{L}$ and the elements $t_{1}, t$ are inside $T$.

Both the image of $\phi$ and $t_{1}$ are contained in $C$. Let $C^{\prime} \subset L^{\prime}$ denote its derived group. If the semisimple rank of $C$ would be strictly smaller than that of $L$, there would exist a Levi group $N$ such that $C \subset N \varsubsetneqq L$, a contradiction. Hence $C^{\prime}$ has maximal rank in $L^{\prime}$.

Choose $s_{L}$ in the intersection $t_{1} T^{L} \cap L^{\prime}$. By the above, $s_{L}$ is in $T_{L, u}$, the compact form of the maximal torus $T_{L}:=\left(L^{\prime} \cap T\right)^{0}$ of $L^{\prime}$. We put $r_{L}=s_{L} c \in L^{\prime}$, and we claim that this is a $R_{L}$-residual point of $T_{L}$. By Proposition 8.1 this is equivalent to showing that $u$ is a distinguished unipotent element of $C^{\prime}=C_{L^{\prime}}\left(s_{L}\right)$. This means that we have to show that $C_{L^{\prime}}\left(s_{L}, \phi\right)$ does not contain a nontrivial torus. But $L=C_{G}\left(T^{L}\right)$ with $T^{L}$ a maximal torus in $C_{G}\left(t_{1}, \phi\right)$. Hence $C_{G}\left(t_{1}, \phi\right)^{0} \cap L=T^{L}$, and thus

$$
C_{G}\left(s_{L}, \phi\right)^{0} \cap L^{\prime}=C_{G}\left(t_{1}, \phi\right)^{0} \cap L^{\prime}=T_{L} \cap T^{L},
$$

proving the claim.

This proves that $M:=t T^{L}=r_{L} T^{L} \subset T$ is a residual coset, by application of Proposition 7.4.

Notice that (8.3) shows that $T^{L}$ is also a maximal torus of $C_{G}\left(s_{L}, \phi\right)$, and thus of $C_{G}\left(s_{L}, u\right)$.

Finally notice that the $W_{0}$-orbit of the pair $(t, M)$ is uniquely determined by the conjugacy class of $(t, u)$ by the above procedure. We have shown:

Proposition 8.4. For every pair $(t, u)$ with $t$ semisimple and $u$ unipotent satisfying (8.1), we can find a homomorphism $\phi$ as in 8.2 such that $t$ commutes with $c$. Let $T^{L}$ be a maximal torus of $C_{G}\left(t_{1}=t c^{-1}, u\right)$ and put $M=t T^{L}$. By suitable conjugation we can arrange that $t, c$ and $M$ are in $T$. Then $M \subset T$ is a residual coset. If we write $t=r t^{L}$ with $r=s c \in T_{L, u} T_{L, r s}$ and $t^{L} \in T^{L}$, then $T^{L}$ is also a maximal torus of $C_{G}(s, c, u)$. The $W_{0}$-orbit of the pair $(t, M)$ is uniquely determined by $(t, u)$.

Corollary 8.5. There is a one-to-one correspondence between conjugacy classes of pairs $(t, u)$ satisfying (8.1) and $W_{0}$-orbits of pairs $(t, M)$ with $M \subset T$ a residual coset, and $t \in M$.

Proof. The maps between these two sets as defined in Proposition 8.3 and Proposition 8.4 are clearly inverse to each other. 
ON THE SPECTRAL DECOMPOSITION OF AFFINE HECKE ALGEBRAS 129

Remark 8.6. Let $(c, u)$ (with $c \in T_{r s}$ ) be a pair satisfying (8.1), with $u$ a distinguished unipotent element of $G$. Then $u$ will be distinguished in $C_{G}(s)$ for each $s$ in the finite group $C_{G}(c, u)$. In particular, $C_{G}(s)$ is semisimple. Hence s gives rise to a residual point $c s^{\prime}$ in $T$ where $s^{\prime} \in T$ is conjugate with $s$ in $G$. This defines a one-to-one correspondence between the orbits in $C_{G}(c, u)$ with respect to the normalizer $N_{G}\left(C_{G}(c, u)\right)$ and the residual points in $T$ with split part $c$.

The Kazhdan-Lusztig parameters for irreducible representations of $\mathcal{H}$ consist of triples $(t, u, \rho)$ where $(t, u)$ is as above, and $\rho$ is an irreducible representation of the finite group

$$
A(t, u)=C_{G}(t, u) /\left(Z_{G} C_{G}(t, u)^{0}\right),
$$

where $Z_{G}$ is the center of $G$. However, not all the irreducible representations of $A(t, u)$ arise, but only those representations of $A(t, u)$ which appear in the natural action of $A(t, u)$ on the homology of the variety of Borel subgroups of $G$ containing $t$ and $u$.

Moreover, Kazhdan and Lusztig show that the irreducible representation $\pi(t, u, \rho)$ is tempered if and only if $t \in M^{t e m p}$, where $M$ is the residual subspace associated to the pair $(t, u)$. In this way we obtain a precise geometric description of the set of minimal central idempotents $\left\{e_{i}\right\}_{i=1}^{l_{t}}$ of the residue algebra $\mathcal{H}^{t}$ for $R_{M}$-generic $t \in M^{t e m p}$.

\section{References}

[1] J. Arthur, Eisenstein series and the trace formula, Proc. of Symp. in Pure Math., AMS, 33 (1979), pp. 253-254.

[2] J. Arthur, On elliptic tempered characters, Acta. Math., 171 (1993), pp. 73138.

[3] J. Arthur, A Paley-Wiener theorem for real reductive groups, Acta. Math. 150 (1983), pp. 1-89.

[4] E.P. van den Ban, J. Carmona, P. Delorme, Paquets d'ondes dans l'espace de Schwartz d'un espace symétrique réductif, J. Funct. Anal., 139 (1996), pp. 225-243.

[5] E.P. van den Ban, H. Schlichtkrull, A residue calculus for root systems, Comp. Math. 123, (2000), pp. 27-72,

[6] E.P. van den Ban, H. Schlichtkrull, Fourier inversion on a reductive symmetric space, Acta. Math. 182 (1999), pp. 25-85.

[7] D. Barbasch, A. Moy, Reduction to real infinitesimal character in affine Hecke algebras, Journal of the AMS 6(3) (1993), pp. 611-630.

[8] A. Borel, J. de Siebenthal, Les sous-groupes fermés de rang maximum des groupes de Lie clos Comment. Math. Helv 23 (1949), pp. 200-221.

[9] R. Bott, An application of the Morse theory to the topology of Lie groups, Bull. Soc. Math. France, 84 (1956), 251-282. 
[10] R.W. Carter, Finite groups of Lie type Wiley Classics Library, John Wiley and sons, Chichester, UK.

[11] W. Casselman, Introduction to the theory of admissible representations of $p$ adic reductive groups, mimeographed notes.

[12] P. Delorme, Espace de coefficients de répresentations admissibles d'un groupe réductif $p$-adique preprint (2003), to appear.

[13] P. Delorme, E.M.Opdam, The Schwartz algebra of an affine Hecke algebra, in preparation.

[14] J. Dixmier, Les algèbres d'opérateurs dans l'espace Hilbertien Cahiers scientifiques, fasc. XXV (1969), Gauthier-Villars, Paris.

[15] J. Dixmier, Les $C^{*}$-algèbres et leurs representations Cahiers scientifiques, fasc. XXIX (1969), Gauthier-Villars, Paris.

[16] J.M.G. Fell, R.S. Doran, Representations of *-Algebras, Locally Compact Groups, and Banach *-Algebraic Bundles Pure and Applied Mathematics 125, Academic Press.

[17] Harish-Chandra, Automorphic forms on semi-simple Lie groups, LN 62 (1968), Springer Verlag.

[18] G.J. Heckman, E.M. Opdam, Yang's system of particles and Hecke algebras, Annals of mathematics 145 (1997), pp. 139-173.

[19] G.J. Heckman, E.M. Opdam, Harmonic analysis for affine Hecke algebras, Current Developments in Mathematics (S.-T. Yau, editor), 1996, Intern. Press, Boston.

[20] V. Heiermann, Décomposition spectrale et représentations spéciales d'un groupe réductif p-adique J. Inst. Math. Jussieu. (to appear).

[21] S.C. Hille, Canonical representations, Thesis University of Leiden (1999).

[22] S. Kato, Irreducibility of principal series representations for Hecke algebras, J. Fac. Sci., U. of Tokyo 28 (1982), pp. 929-943.

[23] D. Kazhdan, G. Lusztig, Proof of the Deligne-Langlands conjecture for affine Hecke algebras, Invent. Math. 87 (1987), pp. 153-215.

[24] R.P. Langlands, On the functional equations satisfied by Eisenstein series, LN 544 (1976), Springer Verlag.

[25] G. Lusztig, Some examples of square integrable representations of semisimple p-adic groups, T.A.M.S. 277 (1983) no. 2, pp. 623-653.

[26] G. Lusztig, Affine Hecke algebras and their graded version, J. Amer. Math. Soc. 2 (1989), no. 3, pp. 599-635.

[27] G. Lusztig, Cuspidal local systems and graded Hecke algebras II, Representations of Groups (B. Allison, G. Cliff editors), Conf. proc. Canad. Math. Soc. 16, Amer. Math. Soc., Providence (1995), pp. 217-275.

[28] G. Lusztig, Classification of unipotent representations of simple p-adic groups, IMRS 11 (1995) pp. 517-589.

[29] G. Lusztig, Cuspidal local systems and graded Hecke algebras, III preprint RT/0108173 (2001).

[30] G. Lusztig, Left cells in Weyl groups Lecture notes in Mathematics 1024, Springer, 1983.

[31] I.G. Macdonald, Spherical functions on a group of p-adic type, Publ. Ramanujan Institute 2 (1971).

[32] I.G. Macdonald, The Poincare series of a Coxeter group, Math. Ann. 199 (1972). 
ON THE SPECTRAL DECOMPOSITION OF AFFINE HECKE ALGEBRAS 131

[33] I.G. Macdonald, A formal identity for affine root systems, preprint 1997.

[34] H. Matsumoto Analyse harmonique dans les systems de Tits bornologiques de type affine, Springer lecture notes 590 (1977).

[35] C. Moeglin, J.-L. Waldspurger, Spectral decomposition and Eisenstein series, Cambridge tracts in Mathematics 113, Cambridge University Press.

[36] L. Morris, Tamely ramified intertwining algebras, Invent. Math. 114 (1993), pp. 233-274.

[37] E.M. Opdam, A generating formula for the trace of the Iwahori-Hecke algebra, Progress in Mathematics 210 (2003), pp. 301-323 (also math.RT/0101006).

[38] A. Ram, J. Ramagge, Affine Hecke algebras, cyclotomic Hecke algebras and Clifford theory, preprint, (1999).

[39] M. Reeder, On the Iwahori spherical discrete series of p-adic Chevalley groups; formal degrees and L-packets, Ann. Sci. Ec. Norm. Sup. 27 (1994), pp. 463-491.

[40] M. Reeder, Formal degrees and L-packets of unipotent discrete series of exceptional p-adic groups, with an appendix by Frank Lübeck, J. reine angew. Math. $\mathbf{5 2 0}$ (2000), pp. 37-93.

[41] M. Reeder, Isogenies of Hecke algebras and Langlands correspondence for ramified principal series representations, Representation Theory 6 (2002), pp. 101126.

[42] P. Schneider, U. Stuhler, Representation theory and sheaves on the Bruhat-Tits building Publ I.H.E.S. 85 (1997), pp. 97-191.

[43] L. Schwartz, Théorie des distributions I, Hermann, Paris.

[44] T. Shoji, On the Springer correspondents of the Weyl groups of classical algebraic groups, Comm. in Alg. 7 (1979), no. 16, 1713-1745; correction on 20272033.

[45] K. Slooten, A combinatorial generalization of the Springer correspondence for classical type, Thesis, Universiteit van Amsterdam, 2003.

[46] K. Slooten, private communication.

[47] R. Steinberg, Endomorphisms of linear algebraic groups, Mem. Amer. Math. Soc. 80 (1968).

[48] R. Steinberg, On a theorem of Pittie, Topologie 14 (1975), pp. 173-177.

[49] J.L. Waldspurger, La formule de Plancherel d'après Harish-Chandra Journal de l'Institut de Mathematiques de Jussieu 2 (2003).

[50] A.J. Wassermann, Une démonstration de la conjecture de Connes-Kasparov pour les groupes de Lie linéaires connexes réductifs, C.R. Acad. Sc. Paris 304 No. 18 (1987), pp. 559-562.

[51] A.J. Wassermann, Cyclic cohomology of algebras of smooth functions on orbifolds, In: Operator Algebras and Applications 1, Lond. Math. Soc. Lect. Note Series 136 (1988), pp. 229-251. 


\section{Index}

$(\cdot, \cdot)$

inner product on $\mathcal{H}, 13$

inner product on $\operatorname{Pol}\left(\operatorname{End}\left(\mathcal{V}_{(\mathcal{K} \backslash \Xi)}\right)\right)$, 90

$(x, y)_{t}=\chi_{t}\left(x^{*} y\right)$, semi-definite Hermitian form on $\mathcal{H}^{t}, 73$

$\|\cdot\|_{o}$, operator norm on $\mathcal{H}, 14$

$\sharp$, the *-operator of $\mathcal{H}^{L}, 75$

$f \rightarrow \bar{f}$, average of a section of $\operatorname{End}\left(\mathcal{V}_{\Xi}\right)$, 89

$h \rightarrow h^{*}$, conjugate linear anti-involution of $\mathcal{H}, 13$

$t \rightarrow t^{*}$, anti-holomorphic involution on $T, 44,120$

$\langle\cdot, \cdot\rangle$

rational inner product on $X, Y, 9$, 31

Euclidean inner product on $T_{r s}, 41$ inner product on $V_{\pi}, 66$

$1_{P}$, idempotent in $\mathcal{A}^{a n}(U)$, with support $U_{P}, 64$

$1_{\varpi}$, idempotent in $\mathcal{A}^{a n}(U)$ with support $U_{\varpi}, 63$

$\Gamma$, set of all pairs $\left(\mathcal{R}_{P}, W_{P} r\right)$ with $P \in$ $\mathcal{P}$ and $W_{P} r$ an orbit of residual points in $T_{P}, 81$

$\Gamma_{a}$, complete set of representatives of the association classes in $\Gamma, 83$

$\Delta$, Weyl denominator, 29

$\Delta=\cup_{\gamma \in \Gamma} \Delta_{\gamma}, 83$

$\Delta_{\gamma}$, fiber at $\gamma \in \Gamma$ of the surjection $\Delta \rightarrow \Gamma, 83$

$\Delta_{P}$, fiber at $P \in \mathcal{P}$ of the surjection $\Delta \rightarrow \mathcal{P}, 83$

$\Delta_{\mathcal{R}, W_{0} r}$, irreducible discrete series representations of $\mathcal{H}(\mathcal{R}, q)$ with central character $W_{0} r, 25-26,55$

$\Delta_{\mathcal{R}}\left(=\Delta_{\mathcal{R}, q}\right)$, irreducible discrete series of $\mathcal{H}(\mathcal{R}, q), 55$

$\Delta_{\varpi_{1}, \varpi_{2}}: \mathcal{H}^{\varpi, a n}\left(U_{\varpi}\right) \rightarrow 1_{\varpi_{1}} \mathcal{H}^{a n}(U) 1_{\varpi_{2}}$, linear isomorphisms, 63,74

$\Lambda$, disjoint union of all triples of the form $\left\{\left(\mathcal{R}_{P}, W_{P} r, t\right)\right\}, 81$

$\Lambda^{g e n}=\cup \Lambda_{\gamma}^{g e n}, 81$
$\Lambda_{\mathcal{R}_{P}, W_{P} r}=\Lambda_{\gamma}$, triples in $\Lambda$ with $\gamma=$ $\left(\mathcal{R}_{P}, W_{P} r\right) \in \Gamma$ fixed, 81

$\Lambda_{\gamma}^{\text {gen }}$, triples in $\Lambda_{\gamma}$ with $r t R_{P}$-generic, 81

$\Xi=\Lambda \times_{\Gamma} \Delta, 26-27,84$

$\Xi^{\text {gen }}=\Lambda^{\text {gen }} \times_{\Gamma} \Delta, 85$

$\Sigma$, quotient space of $\Lambda$ for the action of $\mathcal{W}, 83$

$\Psi$, left action of $\mathcal{W}$ on $\Delta, 83$

$\Psi_{g}: \Delta_{\mathcal{R}_{L}, W_{L} r_{L}} \rightarrow \Delta_{\mathcal{R}_{M}, W_{M} r_{M}^{\prime}}$, bijection induced by $\psi_{g}, 74,83$

$\Omega$, length 0 elements in $W, 10$

$\Omega_{f}=\Omega / Z_{X}, 10$

$\gamma_{L} \in \mathfrak{t}, \operatorname{logarithm}$ of $c_{L}, 31$

$\tilde{\delta}_{g}: V_{\delta} \rightarrow V_{\Psi_{g}(\delta)}$, unitary isomorphism intertwining $\delta \circ \psi_{g}$ and $\Psi_{g}(\delta), 84$

$\theta_{x}$, basis elements of $\mathcal{A}, 15$

$\iota_{s}$, intertwining element of $\mathcal{H}, 58$

$\iota_{w}^{0}$, normalized intertwining element of $\mathcal{F} \mathcal{H}, 59$

$\kappa_{L}$, rational number $\int_{\xi_{L}} \omega_{L}, 49$

$\kappa_{\mathcal{R}_{L},\left\{r_{L}\right\}}$, see also $\kappa_{L}$

$\bar{\kappa}_{W_{L} L}$, rational factor in $\nu_{L}$; average of $\kappa_{L}, 23-24,49$

$\bar{\kappa}_{W_{0} r}\left(=\bar{\kappa}_{\mathcal{R}, W_{0} r}\right)$, rational factor in $\nu(\{r\})$, $23-24,51$, see also $\bar{\kappa}_{W_{L} L}$

$\lambda_{\delta}\left(=\lambda_{\mathcal{R}, \delta}\right):=\bar{\kappa}_{W_{0} r}\left|W_{0} r\right| d_{\delta}$, constant factor in $\mu_{P l}(\{\delta\}), \delta \in \Delta_{W_{0} r}$, $27-28$

$\lambda(h)$, left multiplication by $h \in \mathcal{H}, 13$

$\mu_{P l}$, Plancherel measure on $\hat{\mathfrak{C}}, 22,26$, 89

$\nu$, Plancherel measure of $\overline{\mathcal{Z}}$ on $W_{0} \backslash T$, 23-24, 46

$\nu_{L}$, smooth measure on $L^{\text {temp }}$ such that $\nu=\sum_{L} \nu_{L}, 23-24,48$

$\nu_{c}=\mathfrak{Y}_{c}^{1}$, positive $W_{0}$-invariant measure on $T, 46$

$\xi_{L}$, compact cycle in $\mathcal{B}_{L}\left(r_{L}, \delta\right) \backslash \cup_{m \in \mathcal{M}_{L}}$ $L_{m}, 36$

$\pi$, induction functor on $\mathcal{W}_{\Xi}, 85$

$\pi\left(\mathcal{R}_{P}, W_{P} r, \delta, t\right)=\operatorname{Ind}_{\mathcal{H}^{P}}^{\mathcal{H}}\left(\delta_{t}\right)$, parabolically induced representation, $25-$

26,66 
ON THE SPECTRAL DECOMPOSITION OF AFFINE HECKE ALGEBRAS 133

$[\pi(\xi)]$, class of $\pi(\xi)$ modulo equivalence, 85

$\pi^{a n}, \pi$ extended to $\mathcal{H}^{\text {an }}, 59$

$\pi_{U}^{a n}, \pi$ extended to $\mathcal{H}^{a n}(U), 60$

$\rho(h)$, right multiplication by $h \in \mathcal{H}$, 13

$\tau$, trace functional of $\mathcal{H}, 13$

$\phi_{t}: \mathcal{H}^{P} \rightarrow \mathcal{H}_{P}$, surjective homomorphism, 65

$\chi_{\delta}$, character of $\delta, 56$

$\chi_{t}$, local trace of $\mathcal{H}$, sum (over $c$ ) of densities $d\left(\mathfrak{Y}_{c}^{h}\right) / d \nu$ at $t, 24-26$, 46

$\chi_{\mathcal{R}_{L}, W_{L} r_{L}, \delta, t^{L}}$, character of the induced representation $\pi\left(\mathcal{R}_{L}, W_{L} r_{L}, \delta, t^{L}\right)$, 25-26, 69

$\chi_{t, i}$, irreducible character of $\overline{\mathcal{H}^{t}}, 47$, 56

$\psi_{g}: \mathcal{H}^{L} \rightarrow \mathcal{H}^{M}$, isomorphism for $g \in$ $K_{M} \times W\left(F_{L}, F_{M}\right), 74,83$

$\omega=\frac{d t}{c(t, q) c\left(t^{-1}, q\right)},(n, 0)$-form on $T, 28$,

$A\left(n, \mathcal{R}_{L}, W_{L} r_{L}, \delta, t^{L}\right)$, unitary intertwining operator $\left(n \in W\left(F_{L}, F_{M}\right)\right)$, 79

$\mathcal{A}$, abelian subalgebra of $\mathcal{H}, 15$

${ }_{\mathcal{F}} \mathcal{A}:=\mathcal{F} \otimes_{\mathcal{Z}} \mathcal{A}, \mathcal{F}$ field of fractions of $\mathcal{Z}, 58$

$\mathcal{A}^{\text {an }}(U):=\mathcal{Z}^{\text {an }}(U) \otimes_{\mathcal{Z}} \mathcal{A}$, ring of holomorphic functions on $U \subset T, 59$

$\mathcal{A}^{m e}(U):=\mathcal{F}^{m e}(U) \otimes_{\mathcal{Z}} \mathcal{A}$, ring of meromorphic functions on $U \subset$ $T, 60$

$a=\left(\alpha^{\vee}, k\right)$, affine root, 9

$B(\mathfrak{H})$, bounded linear operators on $\mathfrak{H}$, 13

$\mathcal{B}_{r s}^{L}(\delta)$, ball in $T_{r s}^{L}$, radius $\delta$ and center $e, 36$

$\mathcal{B}_{L}\left(r_{L}, \delta\right)$, ball in $T_{L}$, center $r_{L}$ and radius $\delta, 36$

$\mathcal{C}^{\omega}$, set of centers of $\omega$-residual cosets, 31

$\mathcal{C}^{\mathrm{qu}}$, centers of quasi-residual cosets, 42

$\mathcal{C}_{-}^{\text {qu }}$, quasi-residual centers in $\overline{T_{r s,-}}$, 43
$C^{\infty}\left(\mathcal{V}_{\Xi}\right)=C^{\infty}(\Xi) \otimes \mathcal{V}_{\Xi}, 87$

$C^{\infty}(\Xi)$, space of $C^{\infty}$-functions on $\Xi$, 87

$\mathfrak{C}$, the reduced $C^{*}$ algebra of $\mathcal{H}, 14$

$\mathfrak{C}^{\prime}$, dual of $\mathfrak{C}$ as topological vector space, 15

$\hat{\mathfrak{C}}$, dual (spectrum) of $\mathfrak{C}, 16$

$\hat{\mathfrak{C}}_{\mathcal{O}}$, component of $\hat{\mathfrak{C}}$, the closure of $[\pi]\left(W(\mathcal{O}) \backslash \mathcal{O}^{g e n}\right) \subset \hat{\mathfrak{C}}, 90,104-$ 106

$c=c(t, q)$, Macdonald's $c$-function, 29

$c_{\alpha}$, rank one $c$-function, 29

$c_{L}$, center of $L, 31,122$

$D_{\omega}$, minus the divisor of $\omega$ on $T, 30$

$d_{\delta}\left(=d_{\mathcal{R}, \delta}\right)$, residual degree; degree of $\delta$ in the residual Hilbert algebra $\overline{\mathcal{H}^{r}}, 25-26,56$

$d_{t, i}\left(=d_{W_{0} t, i}\right)$, residual degree; degree of $\chi_{t, i}$ in $\overline{\mathcal{H}^{t}}, 47$, see also $d_{\delta}, 56$

$d t$, holomorphic extension of Haar measure on $T_{u}, 28$

$d^{L} t\left(d_{L} t\right)$, holomorphic extension of normalized Haar measure on $T_{u}^{L}$ $\left(T_{L, u}\right), 32$

$E_{t}$, Eisenstein functional, 30

$E_{t}^{L}$, Eisenstein functional of $\mathcal{H}^{L}, 68$

$E_{L, t_{L}}$, Eisenstein functional of $\mathcal{H}_{L}, 68$

$e_{\mathcal{O}} \in \mathfrak{S}$, central idempotent associated with $\mathcal{O}, 104$

$e_{\varpi}$, image of $1_{\varpi}$ in $\mathcal{H}^{t}, 74$

$e_{i}$, minimal central idempotent of $\overline{\mathcal{H}^{t}}$, 47

$F^{\text {aff }}$, affine simple roots, 9

$F_{0} \subset R_{0}$, simple roots of $R_{0}, 9$

$\mathcal{F}^{m e}(U)$, quotient field of $\mathcal{Z}^{\text {an }}(U), 60$

$\mathcal{F}_{\mathcal{H}}$, Fourier transform on $\mathcal{H}, 26-27$, 87

${ }_{\mathcal{F}} \mathcal{A}$, field of fractions of $\mathcal{A}, 29$

$f_{s}=\log _{\mathbf{q}}(q(s)), 12$

$\mathcal{H}$, affine Hecke algebra, 13

$\mathcal{H}^{*}$, algebraic dual of $\mathcal{H}, 15$

$\mathcal{H}_{0}=\mathcal{H}\left(W_{0},\left.q\right|_{S_{0}}\right) \subset \mathcal{H}, 15$

$\mathcal{H}^{r e}$, Hermitian (or real) elements in $\mathcal{H}, 18$ 
$\mathcal{H}_{+}$, positive elements in $\mathcal{H}, 18$

$\mathcal{H}^{P}=\mathcal{H}\left(\mathcal{R}^{P}, q^{P}\right)$, parabolic subalgebra of $\mathcal{H}, 25-26,64$

$\mathcal{H}_{L}:=\mathcal{H}\left(\mathcal{R}_{L}, q_{L}\right)$, semisimple quotient of $\mathcal{H}^{L}, 25-26,68$

$\mathcal{H}^{m e}(U):=\mathcal{F}^{m e}(U) \otimes_{\mathcal{Z}} \mathcal{H}$, localized

Hecke algebra with meromorphic coefficients, 60

${ }_{\mathcal{F}} \mathcal{H}:=\mathcal{F} \otimes_{\mathcal{Z}} \mathcal{H}, \mathcal{F}$ field of fractions of $\mathcal{Z}, 58$

$\mathcal{H}^{a n}(U):=\mathcal{Z}^{\text {an }}(U) \otimes_{\mathcal{Z}} \mathcal{H}$, the Hecke algebra with coefficients in $\mathcal{Z}^{\text {an }}(U)$, 59

$\mathcal{H}^{\varpi}$, cross product of $\mathcal{H}^{P(\varpi)}$ by $W(\varpi)$, 62

$\mathcal{H}^{t}=\mathcal{H} / \mathcal{I}_{t} \mathcal{H}$, where $\mathcal{I}_{t}$ is the maximal ideal of $W_{0} t$ in $\mathcal{Z}, 68$

$\overline{\mathcal{H}^{t}}$, residual Hilbert algebra at $t, 47$

$\hat{\mathcal{H}}$, space of irreducible $*$-representations of $\mathcal{H}, 16$

$\mathfrak{H}$, Hilbert completion of $\mathcal{H}, 13$

$i\left(V_{\delta}\right)=\mathcal{H}\left(W^{P}\right) \otimes V_{\delta}$ if $\delta \in \Delta_{P}, 87$

$i_{L}$, pole order along $L, 30$

$j_{\epsilon}: \mathcal{H}^{m e}(U) \mapsto \mathcal{H}_{q^{\epsilon}}^{m e}\left(U_{\epsilon}\right)$, "scaling" isomorphism of localized Hecke algebras, 94

$K_{L}$, finite abelian group $T_{L} \cap T^{L}, 31$

$K_{\delta} \subset K_{P}$, isotropy subgroup of $[\delta] \in$ $\left[\Delta_{P}\right], 88$

$\mathcal{K}$, normal subgroupoid of $\mathcal{W}, 87$

$k_{L}$, order of $K_{L}, 32$

$L^{t e m p}$, tempered residual coset, 2324, 31, 122

$\mathcal{L}(L)$, intersection of $T_{L}$ with residual cosets $\supset L, 40$

$\mathcal{L}^{L}$, real projections of residual cosets $\supset L, 40$

$\mathcal{L}_{L}$, dual configuration of $\mathcal{L}^{L}, 40$

$\mathcal{L}^{\omega}$, collection of $\omega$-residual cosets, 30

$\mathcal{L}^{\mathrm{qu}}$, collection of quasi-residual cosets, 42

$l$, length function on $W, 10$

Length multiplicative function, 11

$m$

$m: \Lambda \rightarrow S \subset W_{0} \backslash T$, projection, 81 $m: \Sigma^{g e n} \rightarrow S^{g e n}:=m\left(\Lambda^{g e n}\right) \subset S$, homeomorphism, 83

$m^{L}$, quotient $m_{L} / k_{L} \nu_{\mathcal{R}_{L},\left\{r_{L}\right\}}\left(\left\{r_{L}\right\}\right)$, 23-24, 50

$m_{L}$, density function of $\nu_{L} / \bar{\kappa}_{W_{L} L}, 23-$ 24,48

$m_{\{r\}}\left(=m_{\mathcal{R},\{r\}}\right)$, see also $m_{L}$

$N_{w}$, normalized basis elements of $\mathcal{H}$, 13

$N_{W_{0}}\left(W_{P}\right)$, normalizer of $W_{P}$ in $W_{0}$, 81

$\mathcal{N}$, norm function on $W, 10$

$\mathfrak{N}$, von Neumann algebra completion of $\mathcal{H}, 14$

$n_{\alpha}$, numerator of $c_{\alpha}, 59$

$\mathcal{O}$, orbit of twists of cuspidal representations, 88

$\tilde{\mathcal{O}}$, connected component of $\Xi, 26-27$, 86

$P$, weight lattice, 10

$P(\varpi)=P(t)$, basis of simple roots in $R_{P(t),+}$ where $\varpi=W_{P(t)} t, 61$

$\mathcal{P}$, power set of $F_{0}, 81$

$p_{z}: \hat{\mathfrak{C}} \rightarrow \operatorname{Spec}(\mathcal{Z})$, projection, 17

$\operatorname{Pol}(\Xi)$, space of Laurent polynomials on $\Xi, 87$

$\operatorname{Pol}\left(\operatorname{End}\left(\mathcal{V}_{\mathcal{O}}\right)\right)$, polynomial sections in fiber bundle $\operatorname{End}\left(\mathcal{V}_{\mathcal{O}}\right), 88$

$\operatorname{Pol}\left(\operatorname{End}\left(\mathcal{V}_{\Xi}\right)\right)^{\mathcal{W}}$, space of $\mathcal{W}_{\Xi}$-equivariant sections in $\operatorname{Pol}\left(\operatorname{End}\left(\mathcal{V}_{\Xi}\right)\right), 87$

$\operatorname{Pol}\left(\operatorname{End}\left(\mathcal{V}_{(\mathcal{K} \backslash \Xi)}\right)\right)$, polynomial sections in fiber bundle $\operatorname{End}\left(\mathcal{V}_{(\mathcal{K} \backslash \Xi)}\right), 88$

$\operatorname{Pol}\left(\mathcal{V}_{\Xi}\right)=\operatorname{Pol}(\Xi) \otimes \mathcal{V}_{\Xi}, 87$

$Q$, root lattice, 9

$\mathbf{q}$, base for the labels $q(s), 12$

$q, l$-multiplicative function on $W, 11$

$q^{L}$, restriction of $q$ to $\mathcal{R}^{L}, 12$

$q_{L}$, restriction of $q$ to $\mathcal{R}_{L}, 12$

$q_{a}$, affine root label, 12

$q_{\alpha^{\vee}}$, label for $\alpha^{\vee} \in R_{\mathrm{nr}}^{\vee}, 12$

Quasi residual coset, 42

$\mathcal{R}$, root datum, 9

$\mathcal{R}^{L}$, root datum associated to $L, 11$

$\mathcal{R}_{L}$, semisimple root datum associated to $L, 11$ 
ON THE SPECTRAL DECOMPOSITION OF AFFINE HECKE ALGEBRAS 135

$R_{0} \subset X$, reduced integral root system, 9

$R_{0}^{\vee} \subset Y$, coroot system, 9

$R_{P} \subset R_{0}$, parabolic subsystem, root system of $\mathcal{R}_{P}, 11$

$R_{1}$, system of long roots in $R_{\mathrm{nr}}, 12$

$R_{s, 0}\left(R_{s, 1}\right)$, roots of $R_{0}\left(R_{1}\right)$ vanishing in $s \in T, 117$

$R_{\mathrm{nr}}$, non reduced root system, 12

$R^{\text {aff }}$, affine root system, 9

$R_{ \pm}^{\text {aff }}$, positive (negative) affine roots, 10

$R_{P(t)}=R_{P(\varpi)} \subset R_{0}$, parabolic subsystem associated with $t \in \varpi \subset$ $T, 61$

$r_{L}$, element of $L \cap T_{L}, 23-24,31$

$\operatorname{Rat}^{r e g}(\tilde{\mathcal{O}})$, rational functions on $\tilde{\mathcal{O}}$, regular in an open neighborhood of $\tilde{\mathcal{O}} \simeq T_{u}^{P} \subset T^{P}, 87$

$\operatorname{Rat}^{r e g}\left(\mathcal{V}_{\Xi}\right)=\operatorname{Rat}^{r e g}(\Xi) \otimes \mathcal{V}_{\Xi}, 87$

Rat $^{r e g}(\Xi)$, regular rational functions on $\Xi, 87$

$\operatorname{Rep}_{U}(\mathcal{H})$, category of finite dimensional representations of $\mathcal{H}$ whose $\mathcal{Z}$-spectrum is contained in $U, 60$

Residual coset, 23-24

$S \subset W_{0} \backslash T$, image of $p_{z}, 17,23-24$

$S^{\text {aff }}$, simple reflections of $W^{\text {aff }}, 9$

$S_{0}$, simple reflections of $W_{0}, 9$

$S_{c}$, support of $\mathfrak{X}_{c}, 31$

$S_{c}^{\text {qu }}$, support of $\mathfrak{X}_{c}^{h}, 42$

$\mathfrak{S}$, the Schwartz completion of $\mathcal{H}, 103$

$s_{\alpha}$, reflection in $\alpha, 9$

$s_{a}$, affine reflection in $a, 9$

$T=\operatorname{Hom}_{\mathbb{Z}}\left(X, \mathbb{C}^{\times}\right)$, complex algebraic torus, 16

$T^{L} \subset T$, algebraic subtorus of which $L$ is a coset, 31

$T_{L} \subset T$, algebraic subtorus orthogonal to $L, 31$

$T_{r s}=\operatorname{Hom}\left(X, \mathbb{R}_{+}^{\times}\right)$, real split form of T, 28

$T_{r s,-}$, negative chamber in $T_{r s}, 28$

$T_{r s}^{-}$, anti-dual of the positive chamber $T_{r s,+}, 55$

$T_{u}=\operatorname{Hom}\left(X, S^{1}\right)$, compact form of T, 28
$T_{w}$, basis elements of $\mathcal{H}, 13$

$\mathfrak{t}$, Lie algebra $\operatorname{Lie}\left(T_{r s}\right)=\mathbb{R} \otimes Y, 9$

Tempered coset, 23-24

$U_{P}$, certain $W_{P}$-invariant open set in $T, 64$

$\left(V_{P}, \pi_{P}\right)$, representation of $\mathcal{H}^{P}$ with $V_{P}=1_{P} V, 65$

$\mathcal{V}_{\tilde{\mathcal{O}}}=\tilde{\mathcal{O}}_{\delta} \times i\left(V_{\delta}\right)$, trivial fiber bundle over $\tilde{\mathcal{O}}=\tilde{\mathcal{O}}_{\delta}, 87$

$\mathcal{V}_{\mathcal{O}}:=\tilde{\mathcal{O}}_{\delta} \times \mathcal{K}_{\delta} i\left(V_{\delta}\right), 88$

$\mathcal{V}_{\Xi}$, trivial fiber bundle over $\Xi, 87$

$W$, affine Weyl group, 9

$W_{0}$, Weyl group of $R_{0}, 9$

$W^{\text {aff }}=W_{0} \ltimes Q \subset W, 9$

$W_{t}$, stabilizer in $W_{0}$ of $t \in T, 16$

$W_{P}$, Weyl group of $R_{P}$, parabolic subgroup $W_{0}, 11$

$W_{P(t)}=W\left(R_{P(t)}\right)$, parabolic subgroup associated with $t \in T, 62$

$W^{P}=W_{0} / W_{P}$, set of left cosets $w W_{P}$. If $P \subset F_{0}$, identified with shortest length representatives, 11

$W_{\varpi}$, stabilizer in $W_{0}$ of $\varpi=W_{P(t)} t$, 62

$W(\varpi)=\left\{w \in W_{\varpi} \mid w(P(\varpi))=\right.$ $P(\varpi)\}$, complement of $W_{P(\varpi)}$ in $W_{\varpi}, 62$

$W(P, Q)=\left\{w \in W_{0} \mid w(P)=Q\right\}$, with $P, Q \subset F_{0}, 74$

$W(P)$ for the stabilizer in $W_{0}$ of $P \subset$ $F_{0}, 74$

$W\left(\mathcal{O}_{1}, \mathcal{O}_{2}\right)=\left\{n \in W\left(P_{1}, P_{2}\right) \mid \exists k \in\right.$ $\left.K_{P_{2}}:(k \times n) \in \mathcal{W}\left(\delta_{1}, \delta_{2}\right)\right\}, 26-$ 27,88

$W(\mathcal{O})=W(\mathcal{O}, \mathcal{O}), 26-27,88$

$\mathcal{W}$, groupoid whose set of objects is $\mathcal{P}$, with morphisms $\operatorname{Hom}_{\mathcal{W}}(P, Q)=$ $\mathcal{W}(P, Q):=K_{Q} \times W(P, Q), 26-$ 27,82

$\mathcal{W}(P, Q)=\operatorname{Hom}_{\mathcal{W}}(P, Q), 82$

$\mathcal{W}(P)=\mathcal{W}(P, P), 82$

$\mathcal{W}(\gamma)=\{g \in \mathcal{W} \mid g \gamma=\gamma\}, 83$

$\mathcal{W}_{\Xi}:=\mathcal{W} \times \times_{\mathcal{P}} \Xi$, groupoid of standard induction data, 84

$w_{0}$, longest element of $W_{0}, 16$

$w_{P}$, longest element of $W_{P}, 75$ 
$w^{P}$, longest element of $W^{P}, 76$

$X, Y$, lattices, 9

$X_{L} \supset R_{L}$, lattice of $\mathcal{R}_{L}$, character lattice of $T_{L}, 11,31$

$X^{L}$, character lattice of $T^{L}, 31$

$X^{+} \subset X$, dominant cone, 10

$\mathfrak{X}_{c}$, local contribution to $\int_{t_{0} T_{u}} a \omega, 32$

$\mathfrak{X}_{L}$, contribution to $\int_{t_{0} T_{u}}$ aw supported on $L^{\text {temp }}, 36$

$\mathfrak{X}_{c}^{h}$, local contribution to $a \rightarrow \tau(a h)$ at $c, 42$

$Y_{L} \supset R_{L}^{\vee}$, lattice of $\mathcal{R}_{L}$, cocharacter lattice of $T_{L}, 11$

$\mathfrak{Y}_{c}^{h}$, symmetrized local contribution to $a \rightarrow \tau(a h)$ at $c, 43$

$Z_{X}$, length 0 translations in $W, 10$

$\mathcal{Z}$, the center of $\mathcal{H}, 15$

$\overline{\mathcal{Z}}$, closure of $\mathcal{Z}$ in $\mathfrak{C}, 17$

$\mathcal{Z}^{a n}(U)$, ring of $W_{0}$-invariant holomorphic functions on $U \subset T, 59$

$\mathfrak{Z}$, the center of $\mathfrak{N}, 23$ 
ON THE SPECTRAL DECOMPOSITION OF AFFINE HECKE ALGEBRAS 137

Korteweg-De Vries Institute for Mathematics, Universiteit van Amsterdam, Plantage Muidergracht 24, 1018 TV Amsterdam, The NetherLANDS.

E-mail address: opdam@science.uva.nl 